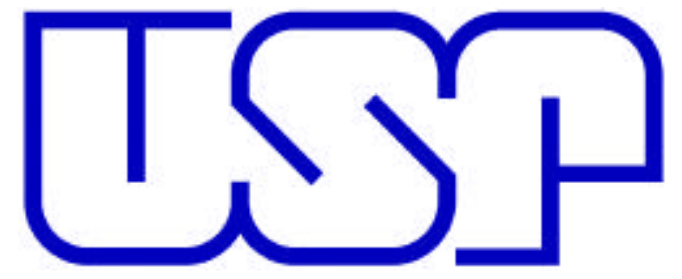

Universidade de São Paulo

Faculdade de Filosofia, Ciências e Letras de Ribeirão Preto Departamento de Química

\title{
APLICAÇÕES DE TÉCNICAS DE RMN À DETERMINAÇÃO ESTRUTURAL DE INTERMEDIÁRIOS SINTÉTICOS
}

\section{VALDEMAR LACERDA JÚNIOR}

Dissertação de Mestrado apresentada à Faculdade de Filosofia, Ciências e Letras de Ribeirão PretoUSP, como parte das exigências para a obtenção do título de Mestre em Ciências - Área de Química 


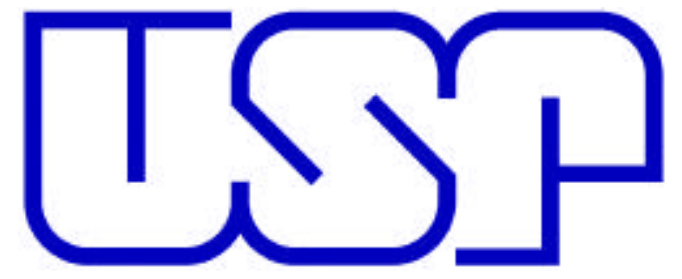

Universidade de São Paulo

Faculdade de Filosofia, Ciências e Letras de Ribeirão Preto Departamento de Química

APLICAÇÕES DE TÉCNICAS DE RMN À DETERMINAÇÃO ESTRUTURAL DE INTERMEDIÁRIOS SINTÉTICOS

\section{VALDEMAR LACERDA JÚNIOR}

Dissertação de Mestrado

Prof. Dr. Mauricio Gomes Constantino

Orientador

RIBEIRÃO PRETO-SP 
Ao meu filho FELIPE de 6 meses de idade

e a minha mulher ANGELA,

pelo amor, amizade, incentivo e apoio;

DEDICO

Aos meus pais WALDEMAR e ANITA,

pelo amor, carinho, compreensão e incentivo;

OFEREÇO 


\section{AGRADECIMENTOS}

- Ao Prof. Dr. Mauricio Gomes Constantino, pela orientação, paciência e amizade.

- Ao Prof. Dr. Gil Valdo José da Silva, pela colaboração e interesse com que acompanhou este trabalho.

- Ao Prof. Dr. Pedro Henrique Ferri e ao Prof. Dr. Silvio do Desterro Cunha, pela amizade, apoio, incentivo e ensinamentos durante minha iniciação ciêntífica.

- À Virgínia pela amizade, paciência, dedicação e esmero com que realizou a obtenção dos espectros de RMN, tão importantes para este trabalho e ao Djalma pela obtenção dos espectros de massas e de infravermelho.

- A todos os meus colegas de Laboratório: Gilberto, Ana, Elaine, Vladimir, Felipe, Adriana, Andréa, Lilian, Barbieri, Miro, Susi, Rosangela, Álvaro, Kleber, Giovanni, Erica, Daniel Borges, Valquíria. 
- Ao Adilson e Daniel(Cobra Criada) pela amizade, ajuda e interesse com que acompanharam este trabalho.

- À toda minha família, que sempre esteve presente em todas as etapas da minha vida.

- Aos funcionários do Departamento de Química que direta ou indiretamente colaboraram para a realização deste trabalho.

- A todas as pessoas que de alguma forma contribuíram para a realização deste trabalho.

- À FAPESP, CAPES e ao CNPq pela ajuda financeira. 


\section{ÍNDICE}

ABREVIATURAS E SÍMBOLOS.

.. $\mathrm{i}$

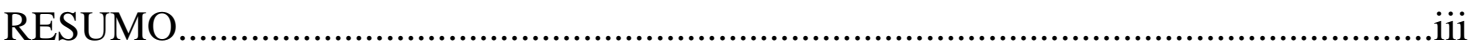

SUMMARY

I.INTRODUÇÃO

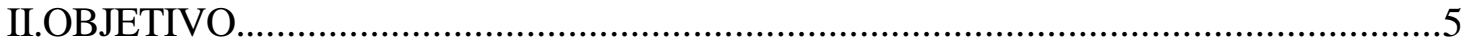

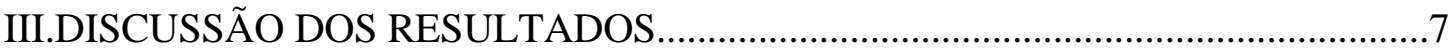

III.1.INTRODUÇÃ

III.2.PREPARAÇÃO DOS COMPOSTOS.......................................................

III.3. DETERMINAÇÃO ESTRUTURAL DOS COMPOSTOS.................................17

III.4.CÁLCULOS DE MODELAGEM MOLECULAR P/OS COMPOSTOS...........106

IV.CONCLUSÃO

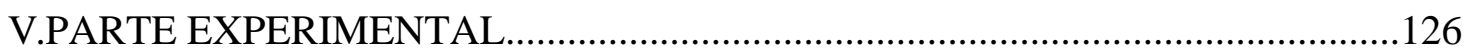

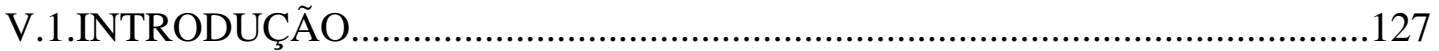

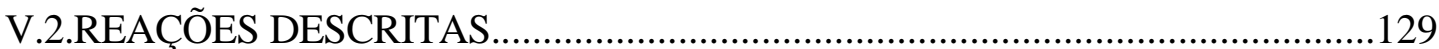

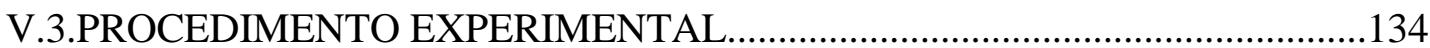

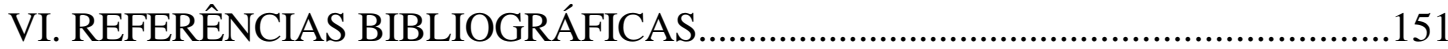




\section{ABREVIATURAS E SÍMBOLOS}

Ac: acetil

AM1: Austin Model 1

BB: Broad Band

ccd: cromatografia em camada delgada

cgl: cromatografia gás-líquido

d: dubleto

dd: duplo dubleto

ddd: duplo duplo dubleto

dddd: duplo duplo duplo dubleto

ddt: duplo duplo tripleto

DEPT: Distortionless Enhancement by Polarization Transfer

$\delta$ : deslocamento químico

$\mathrm{dt}$ : duplo tripleto

EM: Espectro de massas

Et: etil

GMMX: programa Global-MMX

HF: Hartree-Fock

HMBC: Heteronuclear Multiple Bond Correlation

HMQC: Heteronuclear Multiple Quantum Correlation

IV: Infravermelho

$\mathrm{J}$ : constante de acoplamento

$J$-resolved: $J$-resolved spectroscopy

m/z: Relação massa/carga

Me: metil

NOE: Nuclear Overhauser Enhancement

NOESY: Nuclear Overhauser Enhancemente Spectroscopy

OMe: metoxil 
PTSA: Ácido para-toluenossulfônico

Py: Piridina

RMN: Ressonância Magnética Nuclear

${ }^{13} \mathrm{C}$ RMN : Ressonância Magnética Nuclear de Carbono 13

${ }^{1}$ H RMN: Ressonância Magnética Nuclear de Hidrogênio 1

s: singleto

sl: singleto largo

TMS: Tetrametilsilano 


\section{RESUMO}

A conhecida regra do acoplamento em $\mathrm{W}$, que estabelece que núcleos na conformação em $\mathrm{W}$ planar exibem valores significativos de constantes de acoplamento através de quatro ligações, tem sido uma ferramenta útil para a determinação estrutural desde o início do uso da RMN para essa finalidade. Muitas configurações e conformações foram decididas com base nessa regra.

A contínua evolução do equipamento de RMN, porém, resulta em modificações na qualidade e no número dos dados experimentais obtidos, obrigando os químicos a freqüentes revisões de seus pontos de vista sobre a importância relativa dos dados que podem ser obtidos dos espectros de RMN. O equipamento mais recente tem uma resolução maior e várias características adicionais que obscurecem um pouco os conceitos mais antigos: por um lado, o alto valor informativo de algumas técnicas modernas tais como NOE DIFF e outros métodos multi-dimensionais reduzem a importância das constantes de acoplamento; por outro lado, agora é possível determinar um número muito maior de constantes de acoplamento, devido principalmente à maior resolução. Uma consequiência natural é que o químico agora pode explorar o uso de desdobramentos sutis em análise conformacional.

Como parte de nosso trabalho de pesquisa na síntese de produtos naturais, preparamos há algum tempo um número apreciável de derivados de ciclopentanos. Nossa atenção foi fortemente atraída para certas constantes de acoplamento a longa distância $\left({ }^{4} \mathrm{~J}_{\mathrm{HH}}\right)$ que ocorriam em alguns destes compostos, já que uma conformação $\mathrm{W}$ planar não parece ser possível em ciclopentanos. Decidimos então iniciar um estudo mais detalhado dos espectros de RMN desses compostos, com vistas a uma interpretação mais clara dos dados.

Inicialmente fizemos as atribuições para todos os hidrogênios, incluindo a estéreo-química de cada um, e medimos todos os valores de J para os compostos; para tanto fizemos extenso uso dos espectros de RMN tanto de ${ }^{1} \mathrm{H}$ (300 MHz) como de ${ }^{13} \mathrm{C}(75 \mathrm{MHz})$, medidas de efeito NOE, etc. 
Os ângulos entre as ligações e os ângulos diedros (de torção) foram calculados com programas de modelagem molecular; vários programas e métodos diferentes foram usados, para aumentar a confiabilidade.

O primeiro resultado obtido é a confirmação de que ${ }^{4} \mathbf{J}_{\mathrm{HH}} \neq 0$ pode ocorrer mesmo em casos em que uma conformação $\mathrm{W}$ planar não é possível. Mais importante, porém, é a conclusão de que há uma relação entre os valores de ${ }^{4} J_{\mathrm{HH}}$ e os ângulos diedros envolvidos. O acoplamento entre H1 e H2 ocorre através das quatro ligações $\sigma$ definidas pelo caminho $\mathrm{H} 1-\mathrm{C} 1-\mathrm{C} 2-\mathrm{C} 3-\mathrm{H} 2$ e envolve dois ângulos diedros $\theta_{1}$ e $\theta_{2}$. Os valores de ${ }^{4} \mathbf{J}_{\mathrm{HH}}$ foram plotados contra $\left(\cos ^{2} \theta_{1} \times \cos ^{2} \theta_{2}\right.$ ) (uma simples extensão da equação de Karplus); os pontos resultantes não se alinham com perfeição sobre uma curva contínua, mas mostram clara tendência de aumento do valor de ${ }^{4} \mathbf{J}_{\mathrm{HH}}$ conforme os ângulos $\theta_{1} \mathrm{e}$ $\theta_{2}$ se afastam de $90^{\circ}$ e se aproximam de $180^{\circ}$. 


\section{SUMMARY}

The well known W rule, which establishes that nuclei in a planar W arrangement exhibit significant four bond coupling constants, has been a useful tool in molecular structure determination since early times of the use of nmr spectra for this purpose. Many configurations and conformations have been decided based on this rule.

The continuous evolution of the nmr equipment, however, produces modifications in quality and number of available experimental data, thus forcing the chemists to frequent revisions of their points of view about the relative importance of the data that can be obtained from nmr spectra. The more recent equipment has a higher resolution and several additional features that throw some shadow over early concepts: on one hand, the high power of modern techniques such as NOE DIFF and other multi-dimensional methods reduce the importance of coupling constants; on the other hand, it is now possible to determine many more coupling constants, due mainly to the higher resolution. A natural consequence is that the chemist can now exploit the use of subtle splittings in conformational analysis.

As part of our research work on the synthesis of natural products, we have prepared a number of cyclopentane derivatives. Our attention was strongly attracted to the long-range $\left({ }^{4} \mathrm{~J}_{\mathrm{HH}}\right)$ coupling constants that occurred in some of these compounds, as no planar W conformation seems to be possible in cyclopentanes. We have thus decided to start a more detailed study of the nmr spectra of these compounds, seeking for more clear interpretation of the data.

We have first assigned all hydrogens, including the stereochemistry of each hydrogen, and measured all $\mathrm{J}$ values for the compounds; for this task we have used nmr spectra both of ${ }^{1} \mathrm{H}(300 \mathrm{MHz})$ and ${ }^{13} \mathrm{C}(75 \mathrm{MHz}), \mathrm{NOE}$ measurements, etc. 
Bond angles and dihedral (torsion) angles were calculated with molecular modeling programs; several different programs and methods were used to improve reliability.

The first result obtained is a confirmation that a ${ }^{4} \mathrm{~J}_{\mathrm{HH}} \neq 0$ occurs even in cases where a planar W conformation is not possible. More important, however, is the conclusion that there is a correlation between the ${ }^{4} \mathrm{~J}_{\mathrm{HH}}$ values and the involved dihedral angles. There are two dihedral angles in the path through the bonds between two hydrogens which show ${ }^{4} \mathrm{~J}_{\mathrm{HH}}$ coupling. When ${ }^{4} \mathrm{~J}_{\mathrm{HH}}$ values are plotted against $\left(\cos ^{2} \theta_{1} \times \cos ^{2} \theta_{2}\right)$ ( a simple extension of Karplus equation) the points are not aligned over a continuous curve, but they show a clear tendency: ${ }^{4} \mathrm{~J}_{\mathrm{HH}}$ values become higher as the angles $\theta_{1}$ and $\theta_{2}$ vary from 90 to $180^{\circ}$ 


\section{I.INTRODUÇÃO}

Freqüentemente durante a síntese de produtos naturais deparamonos com alguns problemas, principalmente o de determinar a estrutura de um certo intermediário, geralmente um composto novo com estrutura e propriedades totalmente desconhecidas. A determinação da estereoquímica relativa é um aspecto particularmente difícil do problema, sendo freqüentemente necessário recorrer a várias técnicas especiais como COSY, NOESY, HMQC, HMBC, NOE para obter conclusões altamente confiáveis. Por vezes é necessário sintetizar e analisar vários compostos para essa finalidade. ${ }^{1,2}$

Em pesquisas realizadas em nosso laboratório preparamos os compostos da Figura 1 e verificamos que há vários dados analíticos que podem ser obtidos com relativa facilidade, tais como constantes de acoplamento entre hidrogênios vicinais, geminais e a longa distância ${ }^{4} \mathbf{J}_{\mathrm{HH}} \cdot{ }^{3-7}$ Apesar da facilidade de obter tais dados, sua utilidade é reduzida pelo fato de não ser conhecida a relação entre esses dados e a estrutura molecular, na extensão desejável. É uma situação curiosa, em que dados analíticos facilmente obtidos não podem ser traduzidos em informação simples e confiável sobre a estrutura molecular (principalmente do ponto de vista da estereoquímica), apesar de tudo indicar que esses dados contêm a informação requerida. $\mathrm{O}$ que falta, quase certamente, é o conhecimento de algumas das relações entre estrutura molecular e propriedades espectrométricas.

Este desconhecimento ocorre, pelo menos em parte, devido a uma certa dispersão de objetivos de trabalhos destinados a estabelecer as relações entre estruturas e dados espectrométricos; há tantos aspectos diferentes a serem abordados que alguns acabam sendo esquecidos ou negligenciados, e depois fazem falta ao analista que procura resolver certos problemas específicos. 


\section{Figura 1}<smiles>CC1(C)CC(=O)C2OC2(C)C1</smiles><smiles>CC1(C)CC(=O)C(C)(C=O)C1</smiles><smiles>COC(OC)C1(C)CC(C)(C)CC1=O</smiles><smiles>COC(OC)[C@@]1(C)CC(C)(C)C[C@H]1O</smiles><smiles>COC(OC)[C@@]1(C)CC(C)(C)C[C@H]1O</smiles><smiles>COC(OC)[C@]1(C)CC(C)(C)C[C@H]1OC(C)=O</smiles><smiles>COC(OC)[C@]1(C)CC(C)(C)C[C@H]1OC(C)=O</smiles><smiles>CC(=O)O[C@H]1CC(C)(C)C[C@]1(C)C=O</smiles><smiles>CC(=O)O[C@H]1CC(C)(C)C[C@]1(C)C=O</smiles><smiles>CC(=O)O[C@H]1CC(C)(C)C[C@]1(C)C(=O)O</smiles><smiles>CC(=O)O[C@H]1CC(C)(C)C[C@]1(C)C(=O)O</smiles><smiles></smiles><smiles>CC(=O)OC1CC(C)(C)C[C@]1(C)C(C)=O</smiles><smiles>COC(=O)CC(O)[C@]1(C)CC(C)(C)CC1=O</smiles><smiles>CC(=O)CC(O)[C@@]1(C)CC(C)(C)CC1=O</smiles><smiles>CC(=O)CC(O)[C@]1(C)CC(C)(C)C[C@H]1O</smiles><smiles>CC(=O)CC(O)[C@@]1(C)CC(C)(C)C[C@H]1O</smiles><smiles>CC(=O)CC(O)[C@@]1(C)CC(C)(C)C[C@H]1O</smiles><smiles>CC1(C)C[C@H]2OC(=O)C[C@@H](O)[C@]2(C)C1</smiles><smiles>CC1(C)C[C@H]2OC(=O)C[C@@H](O)[C@]2(C)C1</smiles><smiles>CC1(C)C[C@H]2OC(=O)C[C@@H](O)[C@]2(C)C1</smiles> 
A baixa resolução e o número pequeno de recursos disponíveis nos aparelhos de RMN mais antigos também era um fator limitante para quem se propunha a realizar este tipo de estudo. Contudo, os aparelhos mais recentes, de resolução muito mais alta e dotados de inúmeros recursos, permitem ao químico fazer medidas com alto grau de detalhamento e precisão, se tornando hoje uma das técnicas mais poderosas e versáteis ao alcance do químico, sendo usada em pesquisa de nível avançado em diversas áreas, como produtos naturais, síntese orgânica, bioquímica, catalisadores, polímeros etc.

Nesta última década avanços tecnológicos em instrumentação levaram ao aparecimento de muitos novos experimentos de RMN, ${ }^{8}$ alguns dos quais estão se tornando rotineiros. Dentre essas técnicas podemos citar: COSY, NOESY, HETCOR, HMQC, HMBC, NOE DIFF e "J-resolved". 9-11

Esses novos recursos encorajaram-nos a empreender estudos no sentido de determinar novas relações entre estruturas e propriedades espectrométricas, pois estávamos certos de que os espectros produzem mais resultados do que o químico, limitado pelas relações já estabelecidas, consegue utilizar. Inicialmente nossa atenção foi atraída para as constantes de acoplamento a longa distância $\left({ }^{4} \mathrm{~J}_{\mathrm{HH}}\right)$ que ocorrem em alguns dos compostos apresentados na figura 1, em aparente contradição com a regra do acoplamento em W planar, e este é o ponto central do trabalho aqui descrito. 


\section{OBJETIVO}

O objetivo principal do presente trabalho é de procurar estabelecer novas relações entre propriedades espectrométricas (valores de ${ }^{4} \mathrm{~J}_{\mathrm{HH}}$ obtidos experimentalmente) e estrutura molecular (ângulos diedros entre os hidrogênios envolvidos no acoplamento, calculados com o auxílio de programas computacionais). ${ }^{12,13}$

Para tanto, planejamos sintetizar vários intermediários (ciclopentanos substituídos) de sínteses anteriormente realizadas em nosso laboratório ${ }^{18}$ e determinar suas propriedades espectrométricas com a maior precisão possível, fazendo uso de várias técnicas especiais de RMN, tanto de ${ }^{1} \mathrm{H}$ como de ${ }^{13} \mathrm{C}$, tais como HMQC, "J-resolved" e NOE DIFF. 


\section{DISCUSSÃO DOS RESULTADOS}




\section{DISCUSSÃO DOS RESULTADOS}

\section{III.1.INTRODUÇÃO}

Nesta seção serão apresentados os resultados experimentais obtidos, com uma discussão dos aspectos característicos de cada reação, uma análise detalhada dos dados espectroscópicos dos compostos preparados e um estudo de modelagem molecular para os compostos, o que possibilitou correlacionar dados espectroscópicos com estrutura molecular.

A apresentação segue, em princípio, a ordem em que foram feitos os experimentos reais no laboratório.

A identificação dos átomos de carbono e hidrogênio nesta seção não segue nenhuma norma ou recomendação oficial. Os dados de ${ }^{1} \mathrm{H}$ e ${ }^{13} \mathrm{C}$ RMN são apresentados na forma recomendada pela American Society for Testing and Materials. $^{14}$ 
III.2.PREPARAÇÃO DOS COMPOSTOS 


\section{III.2.PREPARAÇÃO DOS COMPOSTOS}

Neste trabalho preparamos vários intermediários sintéticos de sínteses anteriormente realizadas em nosso laboratório. ${ }^{15}$ Procuramos, no entanto, otimizar os resultados, obtendo quantidades suficientes e com pureza adequada para efetuar as análises planejadas . Um resumo das reações é dado a seguir :<smiles>CC1=CC(=O)CC(C)(C)C1</smiles><smiles>CC1(C)CC(=O)C2OC2(C)C1</smiles><smiles>[BH3-][BH3-]</smiles><smiles>CC1(C)CC(=O)C(C)(C=O)C1</smiles><smiles>[CH+]C(=O)CCCCCCCCCC</smiles><smiles>COC(OC)C1(C)CC(C)(C)CC1=O</smiles><smiles>COC(OC)[C@@]1(C)CC(C)(C)C[C@H]1O</smiles><smiles>CO[C@H]1[C@@H](O)CC(C)(C)C[C@H]1OC</smiles><smiles>COC(OC)[C@@]1(C)CC(C)(C)C[C@H]1O</smiles><smiles>COC(OC)[C@]1(C)CC(C)(C)C[C@H]1OC</smiles><smiles>CC(=O)O[C@H]1CC(C)(C)C[C@]1(C)C=O</smiles>

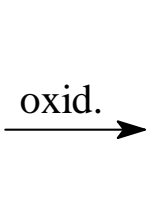<smiles>CC(=O)O[C@H]1CC(C)(C)C[C@]1(C)C(=O)O</smiles><smiles>CCCCC</smiles><smiles>CC(=O)O[C@H]1CC(C)(C)C[C@]1(C)C(C)=O</smiles><smiles>CC1(C)CC(=O)C(C)(C=O)C1</smiles>

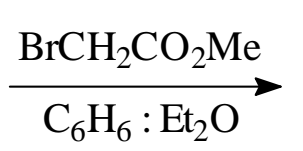<smiles>CC(=O)CC(O)[C@]1(C)CC(C)(C)CC1=O</smiles>
Refluxo<smiles>CC(=O)CC(O)[C@]1(C)CC(C)(C)CC1=O</smiles> 
Os ciclopentanos substituídos foram preparados a partir da isoforona $\mathbf{1}$, que foi inicialmente transformada no epóxido correspondente $\mathbf{2}$ pela reação com $\mathrm{H}_{2} \mathrm{O}_{2}$ a $30 \%$, em meio básico, obtendo-se $68-70 \%$ de um óleo incolor. Durante a reação é necessário fazer um controle rígido da temperatura, principalmente durante a etapa de adição de $\mathrm{NaOH} 6 \mathrm{~N}$, para evitar a formação de sub -produtos.<smiles>CC1=CC(=O)CC(C)(C)C1</smiles>

1

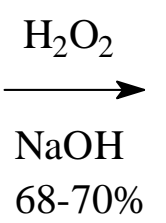

$68-70 \%$

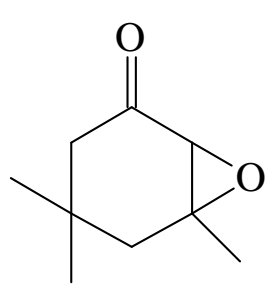

2

Este é o método geralmente utilizado para a preparação de epóxidos de cetonas $\alpha, \beta$-insaturados. A dupla ligação $\mathrm{C}=\mathrm{C}$ desses compostos é pouco nucleofílica devido à conjugação com a carbonila e não reage bem com perácidos. Por isso utiliza-se um reagente nucleofílico como a $\mathrm{H}_{2} \mathrm{O}_{2}$.

O epóxido de isoforona $\mathbf{2}$ foi tratado com ácido de Lewis $\left(\mathrm{BF}_{3} . \mathrm{OEt}_{2}\right)$ para promover o rearranjo com contração de anel, assim formando o ceto-aldeído 3 com $65 \%$ de rendimento.

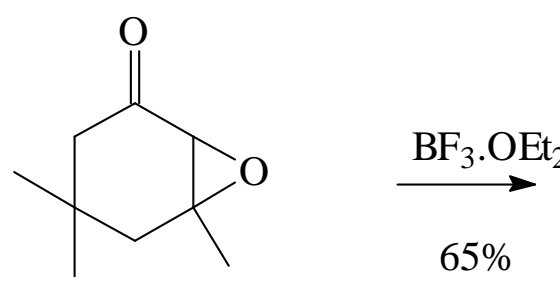

2

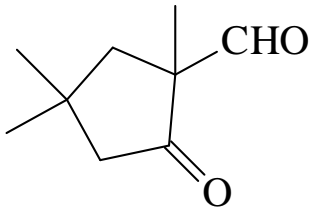

3

A purificação do ceto-aldeído 3 apresentou certa dificuldade devido à sua relativa instabilidade. Além da purificação por cromatografia em coluna de sílica-gel foi necessário destilar o produto em um sistema de destilação 
horizontal $\left(1 \mathrm{mmHg} ; 50^{\circ} \mathrm{C}\right)$ que forneceu o ceto-aldeído 3 com $94 \%$ de pureza por cgl.

Foi feita, então, a proteção da função aldeído para poder reduzir apenas a função cetona. A proteção foi feita com ortoformiato de trimetila, em meio ácido, que forneceu o acetal 4 com $77 \%$ de rendimento.

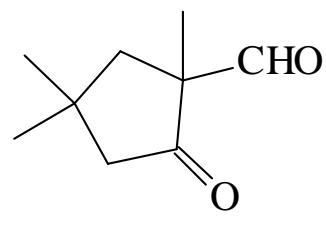

3

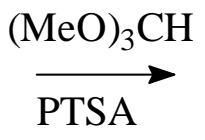

$77 \%$

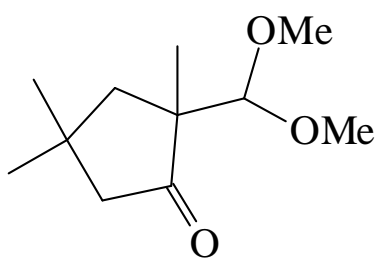

4

O mecanismo dessa reação é o mecanismo que normalmente ocorre nas reações de formação de cetais via carbocátions estabilizados por ressonância (pelo oxigênio); o ortoformiato de trimetila tem também a função de eliminar a água do meio, deslocando o equilíbrio.

$$
\mathrm{HC}(\mathrm{OMe})_{3} \underset{\mathrm{H}_{2} \mathrm{O}}{\stackrel{\mathrm{H}^{\oplus}}{\longrightarrow}} \mathrm{H}-\mathrm{C}_{\mathrm{OMe}}^{\stackrel{\mathrm{O}}{\mathrm{OM}}}+2 \mathrm{MeOH}
$$

Em seguida efetuamos a redução da função cetona do ceto-acetal 4 com $\mathrm{NaBH}_{4}$, obtendo-se um rendimento de $90 \%$ de um óleo amarelo, como uma mistura diastereoisomérica dos compostos cis $\mathbf{5 a}$ e trans $\mathbf{5 b}$, em uma proporção de 1:1.<smiles>COC(OC)C1(C)CC(C)(C)CC1=O</smiles>

4

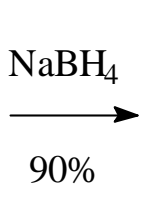<smiles>COC(OC)[C@]1(C)CC(C)(C)C[C@H]1O</smiles>

$5 \mathbf{a}$<smiles>COC(OC)[C@]1(C)CC(C)(C)C[C@H]1O</smiles>

5b

Seria de se esperar que o isômero $\mathbf{5 a}$ fosse formado em maior 
quantidade, pois ele corresponde ao ataque do hidreto pelo lado menos impedido da molécula (controle cinético, que em geral prevalece na redução com hidretos metálicos $)^{16}$.

A presença de um grupo polar como $-\mathrm{CH}(\mathrm{OMe})_{2}$ na vizinhança da cetona pode modificar o resultado, pois alguns hidretos poderiam complexar-se com o grupo polar, fornecendo o ataque do hidreto do mesmo lado desse grupo (ou seja, pelo lado mais impedido da molécula), produzindo um resultado inverso do que foi mostrado anteriormente.

As etapas seguintes da síntese foram realizadas para ambos os isômeros, separadamente.

Inicialmente, foi feita uma acetilação pelo método usual, ${ }^{17,18}$ dos compostos $5 \mathbf{a}$ e $\mathbf{5 b}$, que forneceu os compostos $\mathbf{6 a}$ e $\mathbf{6 b}$ com rendimento de $81 \%$ e $79 \%$, respectivamente.<smiles>COC(OC)[C@]1(C)CC(C)(C)C[C@H]1O</smiles>

$5 \mathbf{a}$<smiles>COC(OC)[C@]1(C)CC(C)(C)C[C@H]1O</smiles>

5b

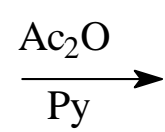

$81 \%$

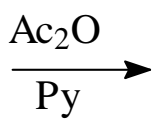

$79 \%$<smiles>COC(OC)[C@]1(C)CC(C)(C)C[C@H]1OC(C)=O</smiles>

$6 \mathbf{a}$<smiles>COC(OC)[C@]1(C)CC(C)(C)CC1OC(C)=O</smiles>

6b

A reação de um álcool com anidrido ou um cloreto de acila é frequientemente o melhor método para a preparação de um éster, sendo que anidridos são menos reativos que os haletos de acila. Ácidos, ácidos de Lewis e bases são freqüentemente usados como catalisadores. ${ }^{19}$ 
A seguir foi feita a hidrólise dos acetais, em condições muito suaves (em sílica contendo solução aquosa de ácido oxálico a 15\%), ${ }^{20}$ obtendo-se os aldeídos-acetatos $\mathbf{7 a}$ e $\mathbf{7 b}$ com rendimentos de $88 \%$ e $84 \%$, respectivamente.<smiles>COC(OC)[C@]1(C)CC(C)(C)C[C@H]1OC(C)=O</smiles>

6a<smiles>COC(OC)[C@]1(C)CC(C)(C)C[C@@H]1OC(C)=O</smiles>

6b<smiles>CC([OH2+])([Os])CCl</smiles>
$88 \%$<smiles>CC(C)(Cl)[O+]S(=O)(=O)O</smiles>
$84 \%$<smiles>CC(=O)O[C@@H]1CC(C)(C)C[C@]1(C)C=O</smiles>

$7 \mathbf{a}$<smiles>CC(=O)O[C@@H]1CC(C)(C)C[C@]1(C)C=O</smiles>

$7 \mathbf{b}$

A oxidação dos aldeídos a ácidos foi realizada sem problemas com $\mathrm{NaClO}_{2},{ }^{21}$ obtendo-se os compostos 8a e 8b com rendimento de $88 \%$ e $91 \%$, respectivamente.<smiles>CC(=O)O[C@@H]1CC(C)(C)C[C@]1(C)C=O</smiles>

$7 \mathbf{a}$<smiles>CC(=O)O[C@H]1CC(C)(C)C[C@]1(C)C=O</smiles>

$7 \mathbf{b}$

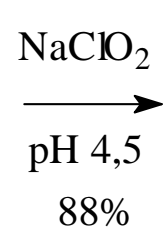

$88 \%$

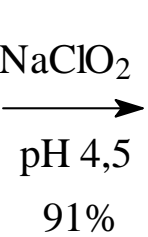

$91 \%$<smiles>CC(=O)O[C@H]1CC(C)(C)C[C@]1(C)C(=O)O</smiles>

8a<smiles>CC(=O)O[C@H]1CC(C)(C)C[C@]1(C)C(=O)O</smiles>

$\mathbf{8 b}$

A purificação normalmente realizada durante a extração, que envolve lavagem da solução aquosa do sal do produto e depois acidulação e extração, mostrou-se suficiente para produzir amostras destinadas a fins analíticos. 
A transformação dos ácidos $\mathbf{8 a}$ e $\mathbf{8 b}$ nos correspondentes ésteres metílicos foi feita por tratamento com diazometano, método que em geral produz ésteres bem puros, em bom rendimento e sob condições suaves, obtendose os compostos 9a e $9 \mathbf{b}$ com $80 \%$ e $84 \%$, respectivamente.<smiles>CC(=O)O[C@H]1CC(C)(C)C[C@]1(C)C(=O)O</smiles>

8a<smiles>CC(=O)O[C@H]1CC(C)(C)C[C@]1(C)C(=O)O</smiles>

$\mathbf{8 b}$

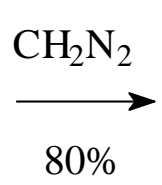

$80 \%$

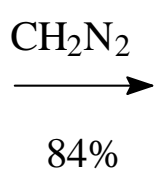

$84 \%$<smiles>CC(=O)O[C@H]1CC(C)(C)C[C@]1(C)C(C)=O</smiles>

9a<smiles>CC(=O)O[C@H]1CC(C)(C)C[C@]1(C)C(C)=O</smiles>

9b

Por último foi feita uma reação de Reformatsky ${ }^{22}$ entre o cetoaldeído 3 e bromoacetato de metila, que forneceu bons resultados. A reação foi químio-e diastereosseletiva; não se detectou nenhum produto resultante do ataque do reagente de Reformatsky à cetona. Formaram-se dois isômeros, 10a e 10b, na proporção de 2:1, com rendimento de $60 \%$.<smiles>CC(=O)C[C@H](O)[C@@]1(C)CC(C)(C)CC1=O</smiles> 
É interessante observar que a estereosseletividade da reação é tal que forma-se principalmente o isômero 10a. Esta diastereosseletividade pode ser explicada se supusermos que os oxigênios das carbonilas do aldeído e da cetona do composto 3 ficam voltados para o mesmo lado (como conseqüência, por exemplo, de uma complexação com o metal, com formação de um anel de seis membros); o reagente de Reformatsky atacaria, desta maneira, a carbonila do aldeído preferencialmente pelo lado menos impedido, levando a formação do isômero 10a em maior quantidade.

Todos os compostos foram caracterizados através de seus espectros de ${ }^{1} \mathrm{H}$ RMN, ${ }^{13} \mathrm{C}$ RMN, IV e CG/MS.

\section{III.3. DETERMINAÇÃO ESTRUTURAL DOS COMPOSTOS}




\section{III.3. DETERMINAÇÃO ESTRUTURAL DOS COMPOSTOS}

Para a determinação estrutural foram feitas análises de ${ }^{1} \mathrm{H}$ RMN, ${ }^{13} \mathrm{C}$ RMN ${ }^{13} \mathrm{C}\left\{{ }^{1} \mathrm{H}\right\}$, DEPT, NOE DIFF, HMQC e "J-resolved" para todos os compostos.

A técnica chamada de HMQC ${ }^{23}$ (Espectroscopia de correlação heteronuclear de quantum múltiplo) se mostrou bastante útil para a determinação estrutural dos compostos, pois correlaciona os picos de um espectro de ${ }^{1} \mathrm{H}$ com os picos de um espectro de ${ }^{13} \mathrm{C}$. Com isto vai além do APT e DEPT, já que mostra os hidrogênios que estão ligados especificamente a cada ${ }^{13} \mathrm{C}$, e até mesmo do HETCOR, pois possui mais resolução e ganha no tempo de aquisição do espectro.

A técnica chamada "J-resolved" ${ }^{24}$ (Espectro Homonuclear 2-D Jresolvido) foi útil mos estudos de determinação das constantes de acoplamento entre os hidrogênios, em especial, nos acoplamentos a longa distância e casos em que hidrogênios estavam sobrepostos. No experimento de 2-D "J-resolved" os valores de deslocamentos químicos e acoplamentos entre os núcleos são separados nos diferentes eixos do espectro de duas dimensões. No eixo $F_{2}$ somente estão presentes as informações a respeito dos deslocamentos químicos, enquanto, que em $F_{1}$ estão as informações sobre os acoplamentos entre os núcleos.

O espectro NOE DIFF ${ }^{23,25}$ é muito útil, tanto na determinação estrutural como no estudo de conformações, porque permite determinar quais grupos de prótons estão próximos entre si. No caso dos compostos que sintetizamos, para aplicar a técnica de NOE DIFF adequadamente, escolhemos posições estratégicas para a irradiação: as 3 metilas (ligadas ao anel ciclopentano). Assim foi possível determinar a posição na molécula e a estereoquímica de todos os hidrogênios.

$\mathrm{Na}$ discussão que se segue, a numeração dos átomos de carbono dos compostos não segue nenhuma norma ou recomendação oficial. O objetivo da numeração dos compostos é facilitar a identificação dos átomos de hidrogênios nas discussões dos espectros. 


\section{Composto (2)}

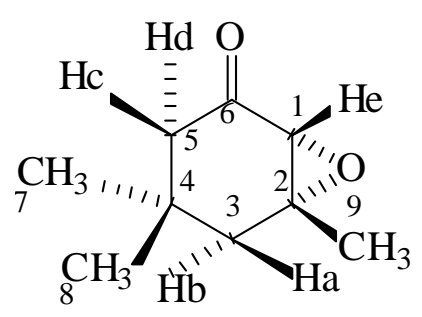

Espectro de ${ }^{1}$ H-RMN do composto (2)

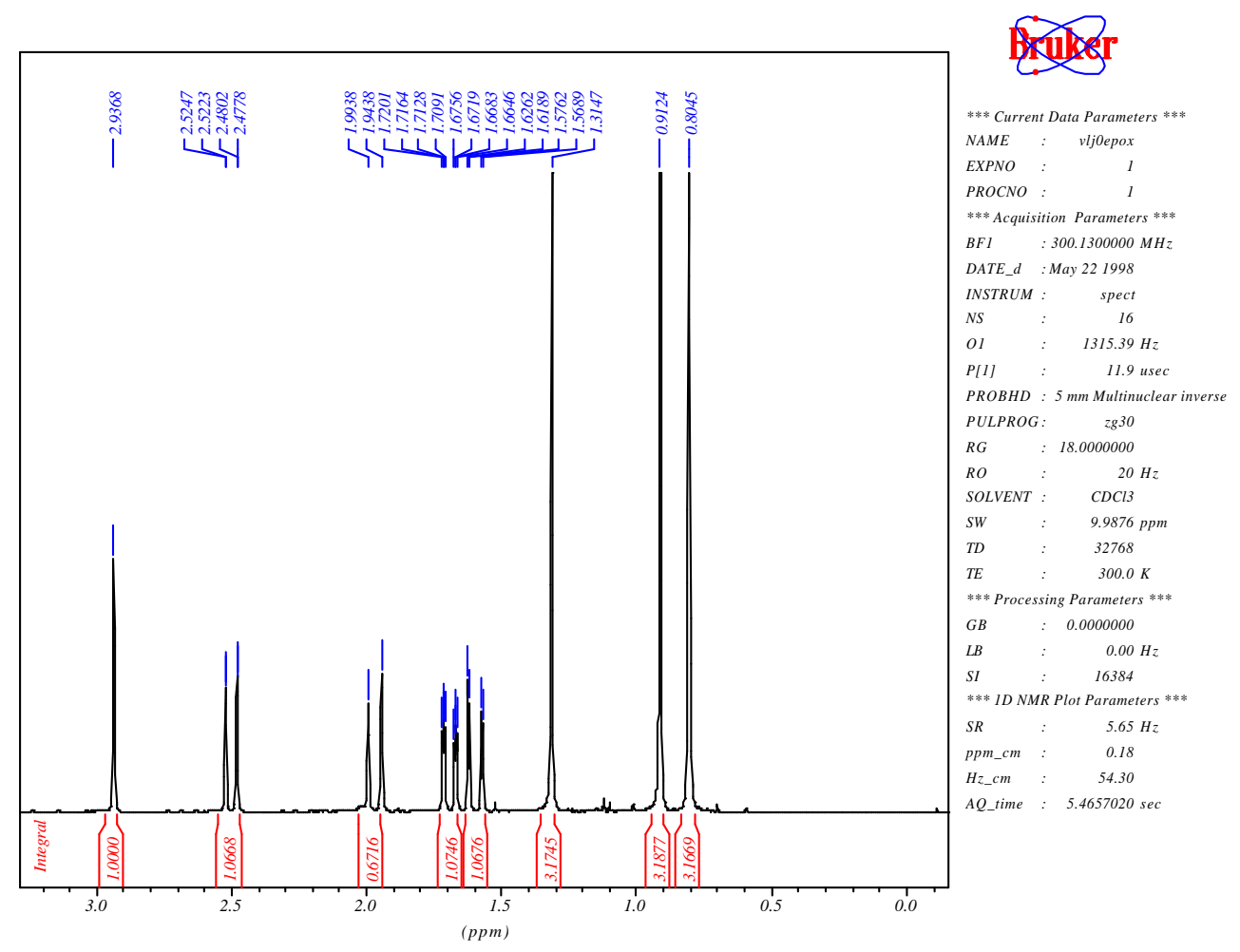

Tabela 1 - Dados espectrais de ${ }^{1} \mathrm{H}$ RMN do composto $2\left(\mathrm{CDCl}_{3}\right)$.

\begin{tabular}{c|l|c|l}
\hline$\delta(\mathbf{p p m})$ & Atribuição & sinal & \multicolumn{1}{|c}{$\mathbf{J}(\mathbf{H z})$} \\
\hline 2,93 & $\mathrm{He}$ & $\mathrm{dd}$ & $\mathrm{Jce}=1,1 \mathrm{e} \mathrm{Jbe}=0,9 \mathrm{~Hz}$ \\
2,50 & $\mathrm{Hd}$ & $\mathrm{dd}$ & $\mathrm{Jdc}=13,4 \mathrm{e} \mathrm{Jdb}=0,9 \mathrm{~Hz}$ \\
1,97 & $\mathrm{Hb}$ & $\mathrm{dt}$ & $\mathrm{Jba}=15 ; \mathrm{Jbe}=\mathrm{Jbd}=0,9 \mathrm{~Hz}$ \\
1,69 & $\mathrm{Hc}$ & $\mathrm{ddd}$ & $\mathrm{Jcd}=13,4 ; \mathrm{Jca}=2,2 \mathrm{e} \mathrm{Jce}=$ \\
& & & $1,1 \mathrm{~Hz}$ \\
1,59 & $\mathrm{Ha}$ & $\mathrm{dd}$ & $\mathrm{Jab}=15$ e Jac=2,2Hz \\
1,30 & $\mathrm{CH}_{3}(9)$ & $\mathrm{s}$ & \\
0,9 & $\mathrm{CH}_{3}(7)$ & $\mathrm{s}$ & \\
0,79 & $\mathrm{CH}_{3}(8)$ & $\mathrm{s}$ & \\
\hline
\end{tabular}




\section{Espectro de ${ }^{13} \mathrm{C}-\mathrm{RMN}$ (BB) do composto (2)}

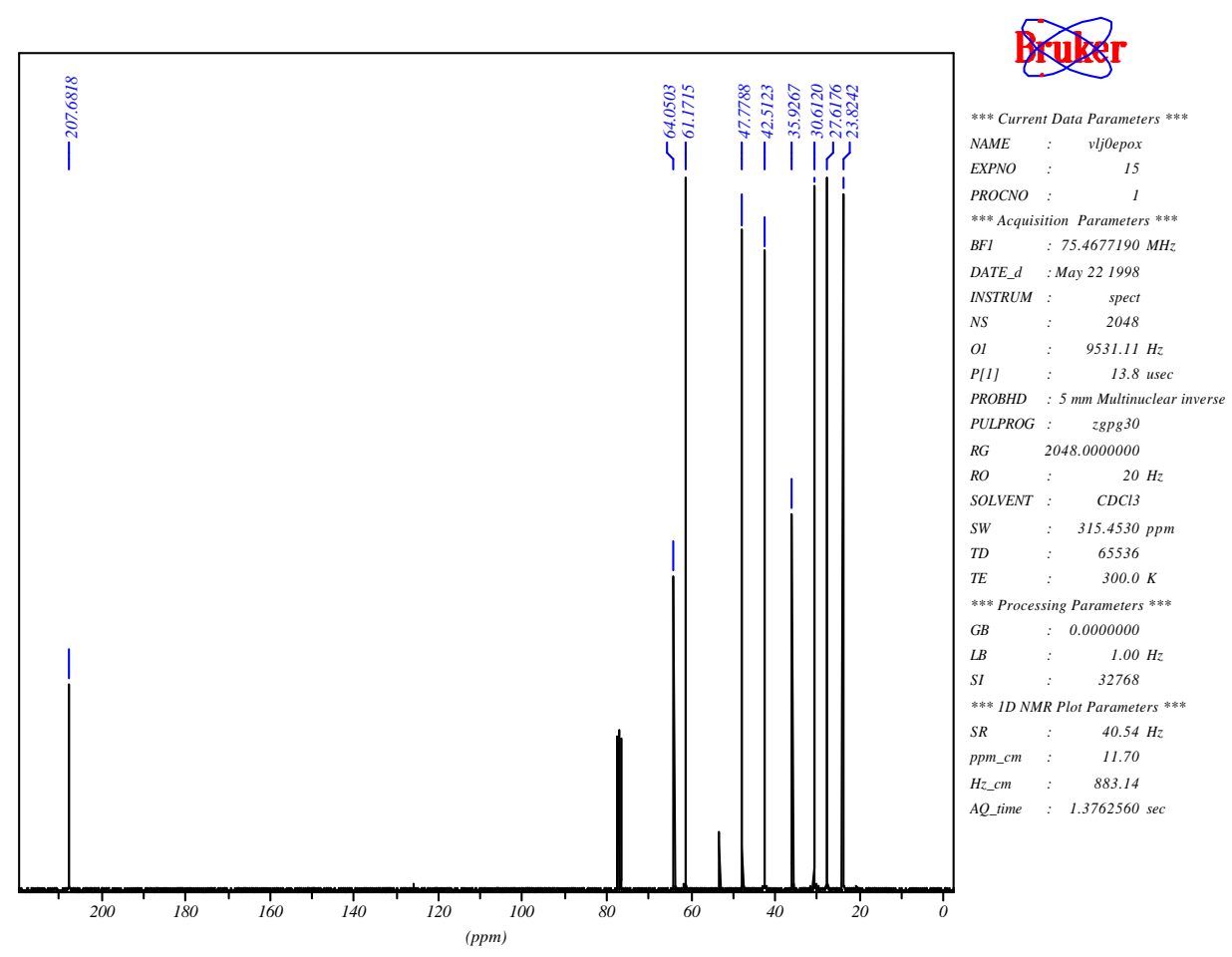

Espectro de ${ }^{13}$ C-RMN (DEPT-135) do composto (2)

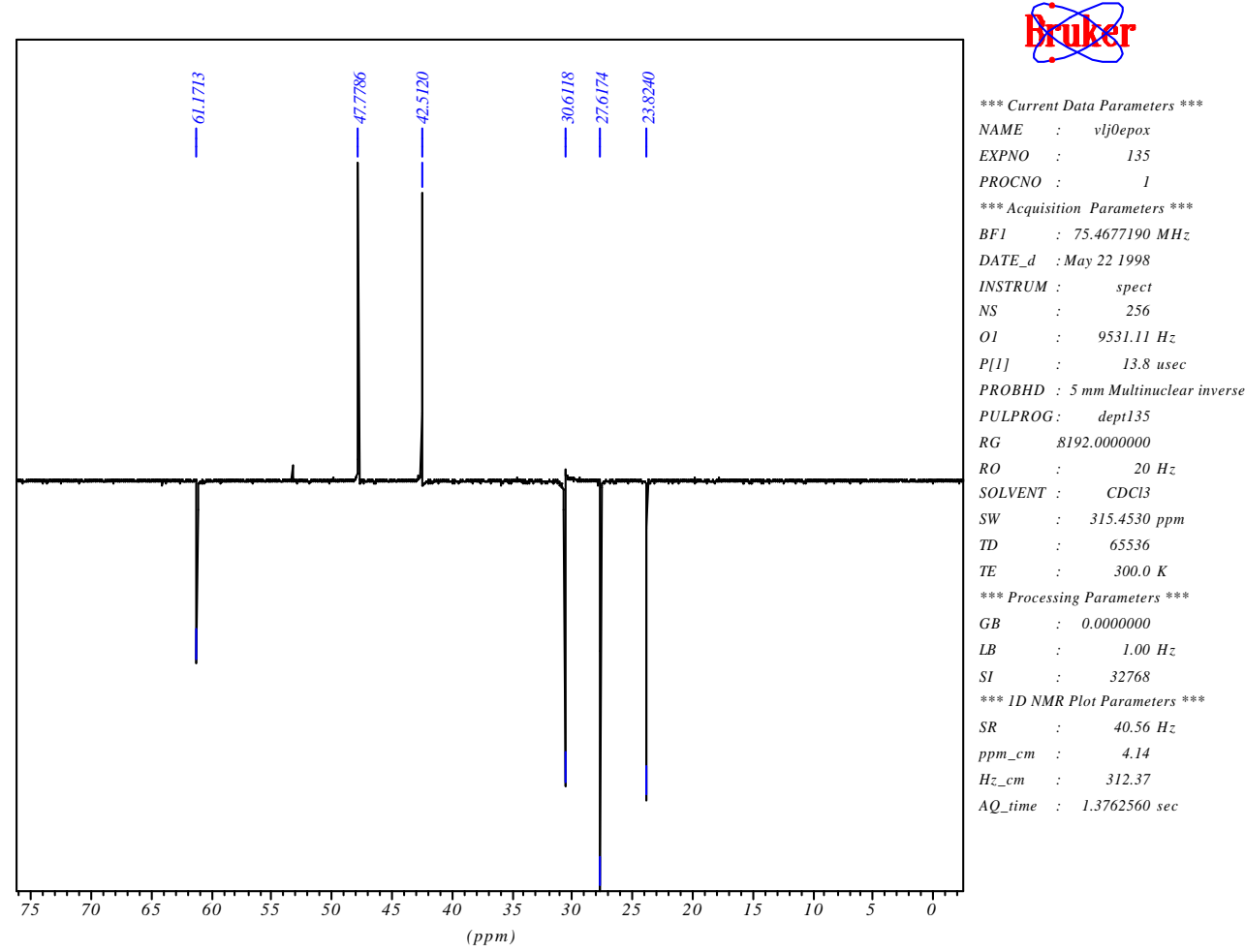




\section{Espectro HMQC (2D H/C) do composto (2)}

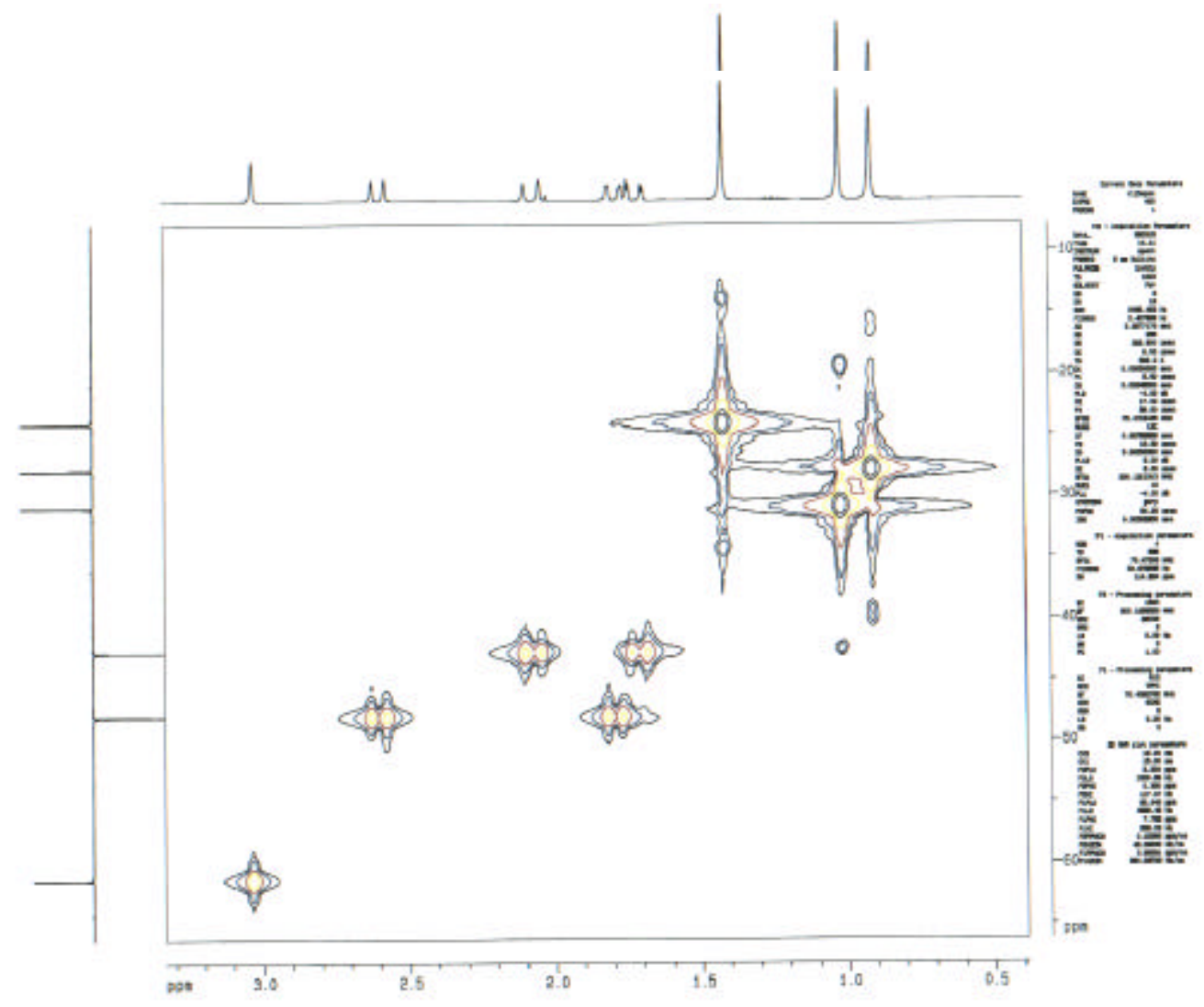

Espectro Jres do composto (2)
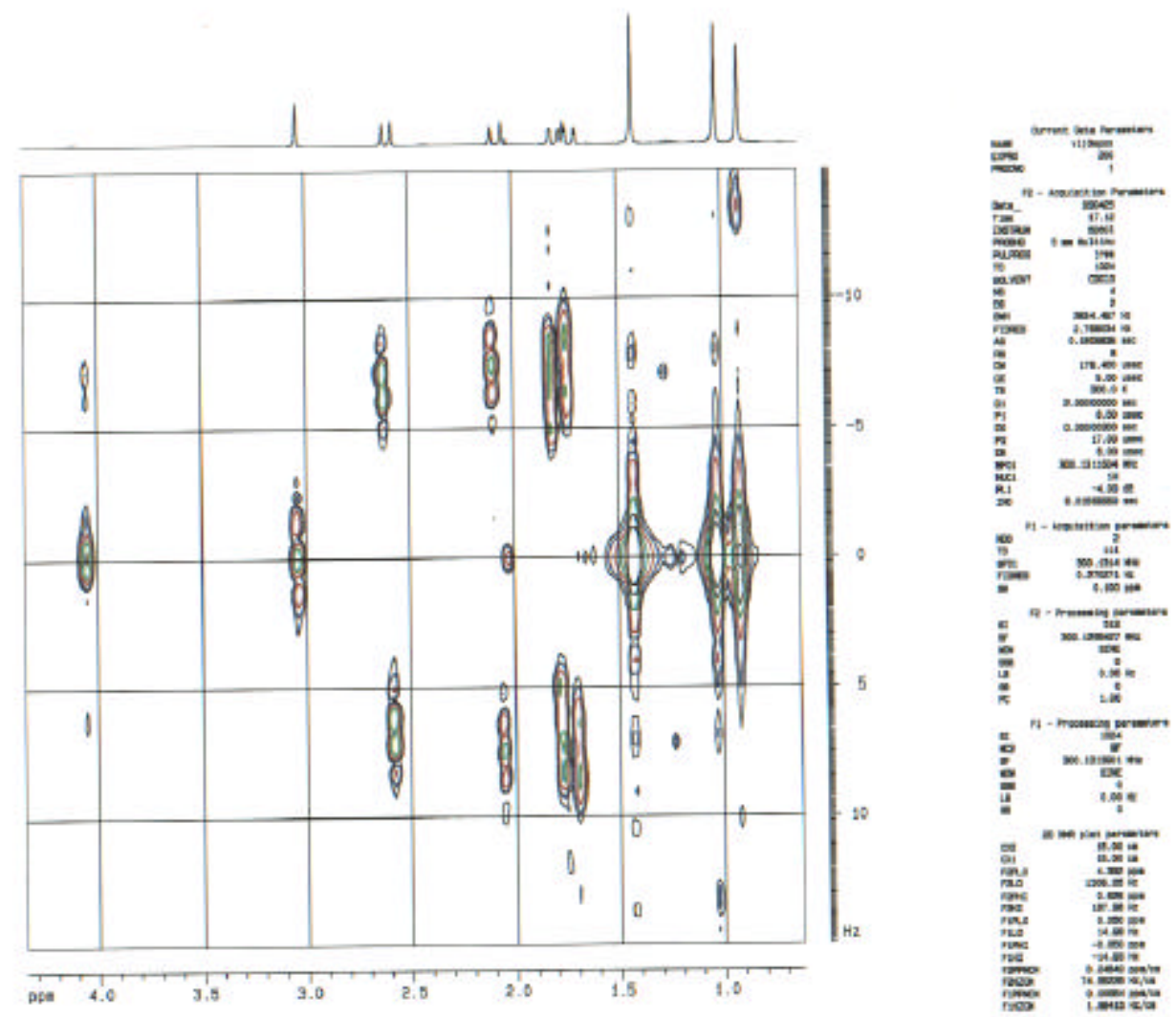


\section{Espectros NOE DIFF do composto (2)}

$>$ irradiação na metila 7

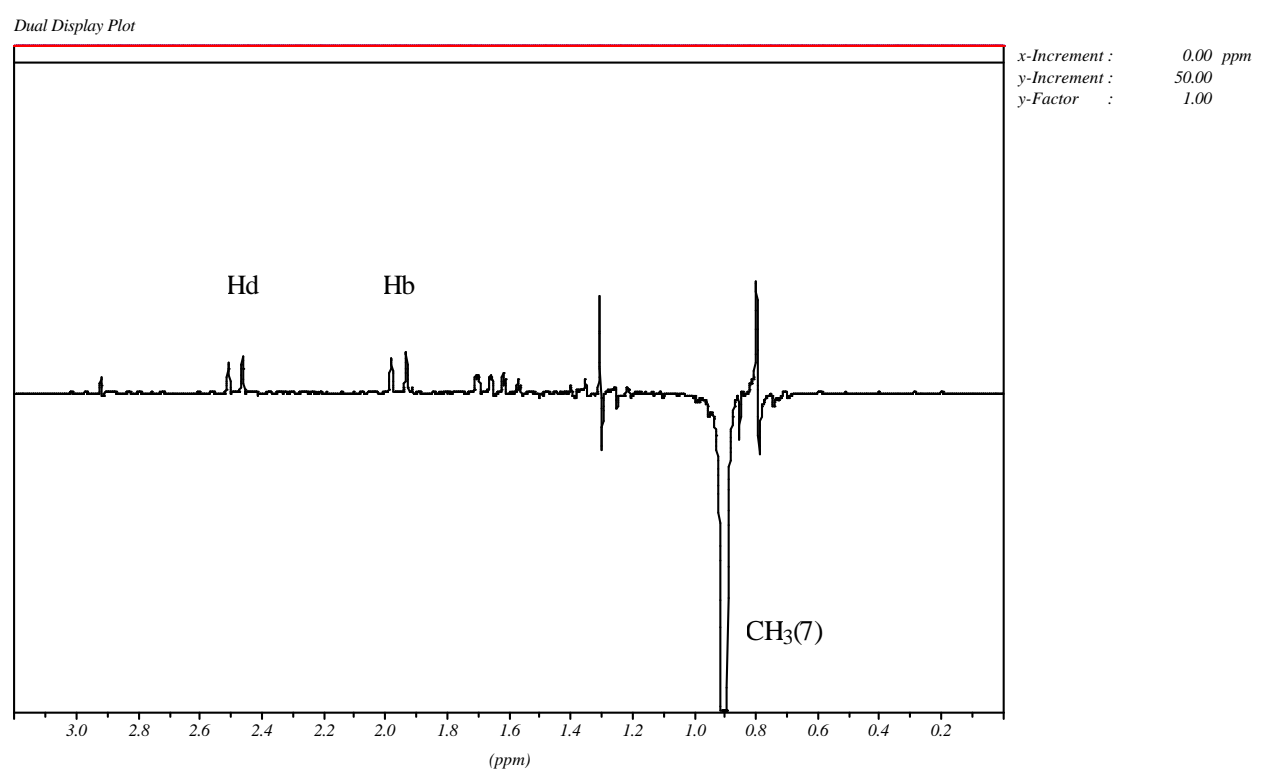

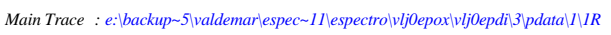

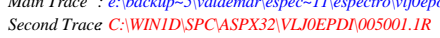

irradiação na metila 8

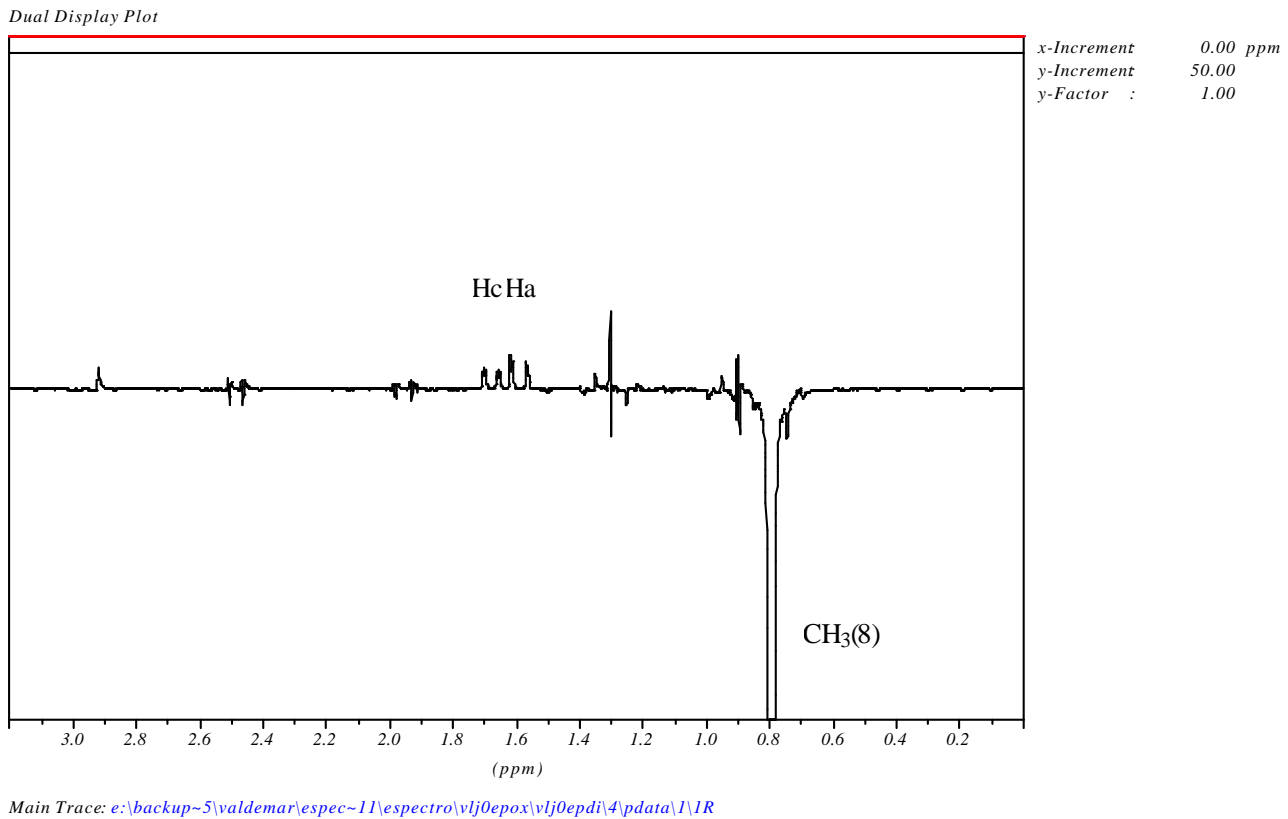

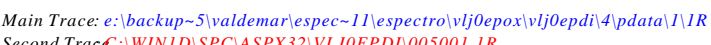

Second Trace: \WINID SPPCASPX32\VLJOEPDI005001.1R 
irradiação na metila 9

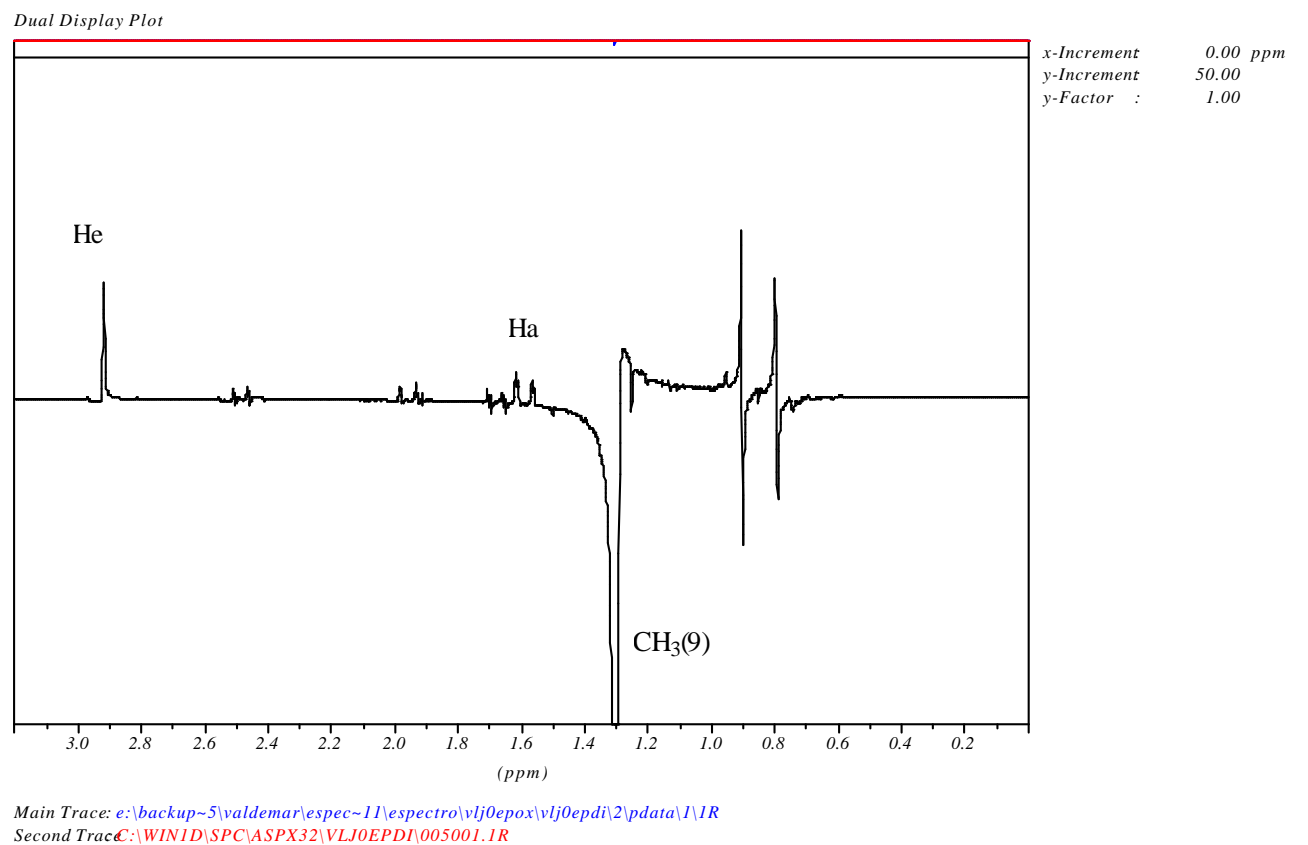

Tabela 2 - Dados espectrais de NOE DIFF do composto $2\left(\mathrm{CDCl}_{3}\right)$.

\begin{tabular}{c|c|c}
\hline$\delta$ (ppm)Irradiado & Atribuição & Efeito NOE Observado \\
& & \\
\hline 1,30 & $\mathrm{CH}_{3}(9)$ & $\mathrm{Ha}, \mathrm{He}$ \\
0,9 & $\mathrm{CH}_{3}(7)$ & $\mathrm{Hb}, \mathrm{Hd}$ \\
0,79 & $\mathrm{CH}_{3}(8)$ & $\mathrm{Ha}, \mathrm{Hc}$ \\
\hline
\end{tabular}

Tabela 3- Dados espectrais de ${ }^{1} \mathrm{H}$ e ${ }^{13} \mathrm{C}$ RMN para $( \pm)$ - 4,4,6-trimetil-7oxabiciclo[4.1.0] heptan-2-ona $\left(\mathrm{CDCl}_{3}\right)$.

\begin{tabular}{|c|c|c|}
\hline $\mathrm{C}-, \mathrm{H}-$ & ${ }^{1} \mathrm{H}(300 \mathrm{MHz}) \delta(\mathrm{ppm})$ & ${ }^{13} \mathrm{C}(75 \mathrm{MHz})$ \\
\hline 1 & $\mathrm{He}-2,935(\mathrm{dd}, 1 \mathrm{H}, \mathrm{Jce}=1,1$ e Jbe $=0,9 \mathrm{~Hz})$ & 61,17 \\
\hline 2 & & 64,05 \\
\hline 3 & $\begin{array}{c}\mathrm{Hb}-1,97(\mathrm{dt}, 1 \mathrm{H}, \mathrm{Jba}=15 ; \mathrm{Jbe}=\mathrm{Jbd}=0,9 \mathrm{~Hz}) \text { e } \mathrm{Ha}-1,59 \\
(\mathrm{dd}, 1 \mathrm{H}, \mathrm{Jab}=15 \text { e Jac }=2,2 \mathrm{~Hz})\end{array}$ & 42,51 \\
\hline 4 & & 35,92 \\
\hline 5 & $\begin{array}{c}\mathrm{Hd}-2,50(\mathrm{dd}, 1 \mathrm{H}, \mathrm{Jdc}=13,4 \text { e } \mathrm{Jdb}=0,9 \mathrm{~Hz}) \text { e } \mathrm{H} \mathrm{c}- \\
1,69(\mathrm{ddd}, 1 \mathrm{H}, \mathrm{Jcd}=13,4 ; \mathrm{Jca}=2,2 \text { e Jce }=1,1 \mathrm{~Hz})\end{array}$ & 47,77 \\
\hline 6 & & 207,68 \\
\hline 7 & $0,9(\mathrm{~s}, 3 \mathrm{H})$ & 30,61 \\
\hline 8 & $0,79(\mathrm{~s}, 3 \mathrm{H})$ & 27,61 \\
\hline 9 & $1,30(\mathrm{~s}, 3 \mathrm{H})$ & 23,82 \\
\hline
\end{tabular}




\section{Composto (3)}

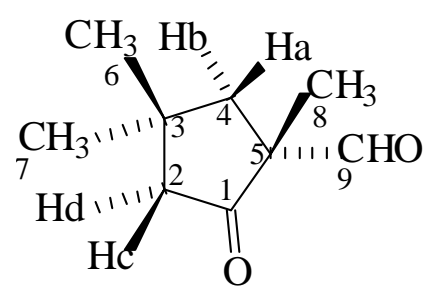

\section{Espectro de ${ }^{1} \mathrm{H}-\mathrm{RMN}$ do composto (3)}

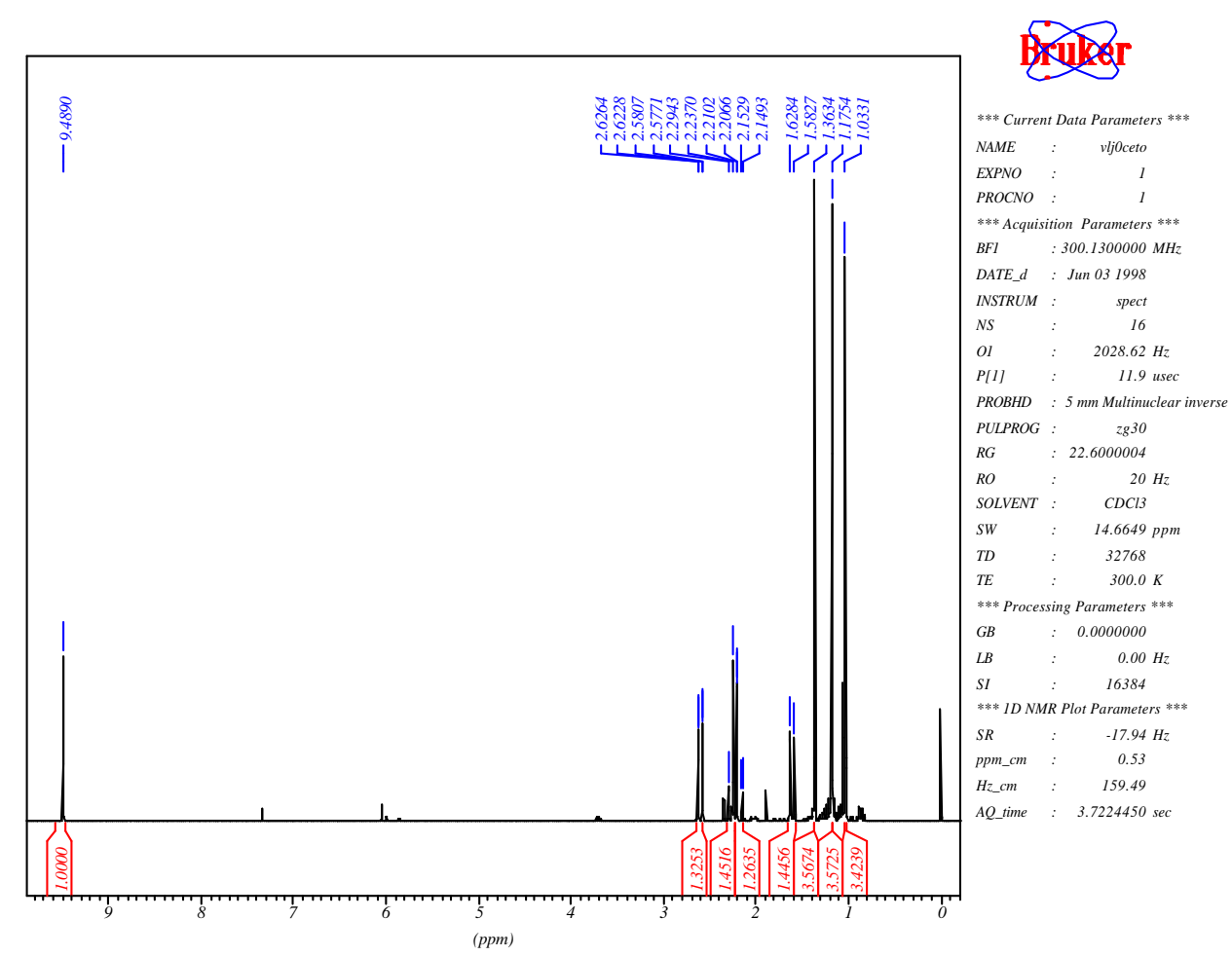

Tabela 4 - Dados espectrais de ${ }^{1} \mathrm{H}$ RMN do composto $3\left(\mathrm{CDCl}_{3}\right)$.

\begin{tabular}{c|c|c|c}
\hline$\delta(\mathbf{p p m})$ & Atribuição & Sinal & $\mathbf{J}(\mathbf{H z})$ \\
\hline 9,48 & 9 & $\mathrm{~s}$ & \\
2,59 & $\mathrm{Hb}$ & $\mathrm{dd}$ & $\mathrm{Jba}=13,7 \mathrm{e} \mathrm{Jbd}=1 \mathrm{~Hz}$ \\
2,27 & $\mathrm{Hc}$ & $\mathrm{d}$ & $\mathrm{Jcd}=17,2 \mathrm{~Hz}$ \\
2,17 & $\mathrm{Hd}$ & $\mathrm{dd}$ & $\mathrm{Jdc}=17,2 \mathrm{e} \mathrm{Jdb}=1 \mathrm{~Hz}$ \\
1,61 & $\mathrm{Ha}$ & $\mathrm{d}$ & $\mathrm{Jab}=13,7 \mathrm{~Hz}$ \\
1,35 & $\mathrm{CH}_{3}(8)$ & $\mathrm{s}$ & \\
1,17 & $\mathrm{CH}_{3}(6)$ & $\mathrm{s}$ & \\
1,03 & $\mathrm{CH}_{3}(7)$ & $\mathrm{s}$ & \\
\hline
\end{tabular}




\section{Espectro de ${ }^{13} \mathrm{C}-\mathrm{RMN}$ (BB) do composto (3)}

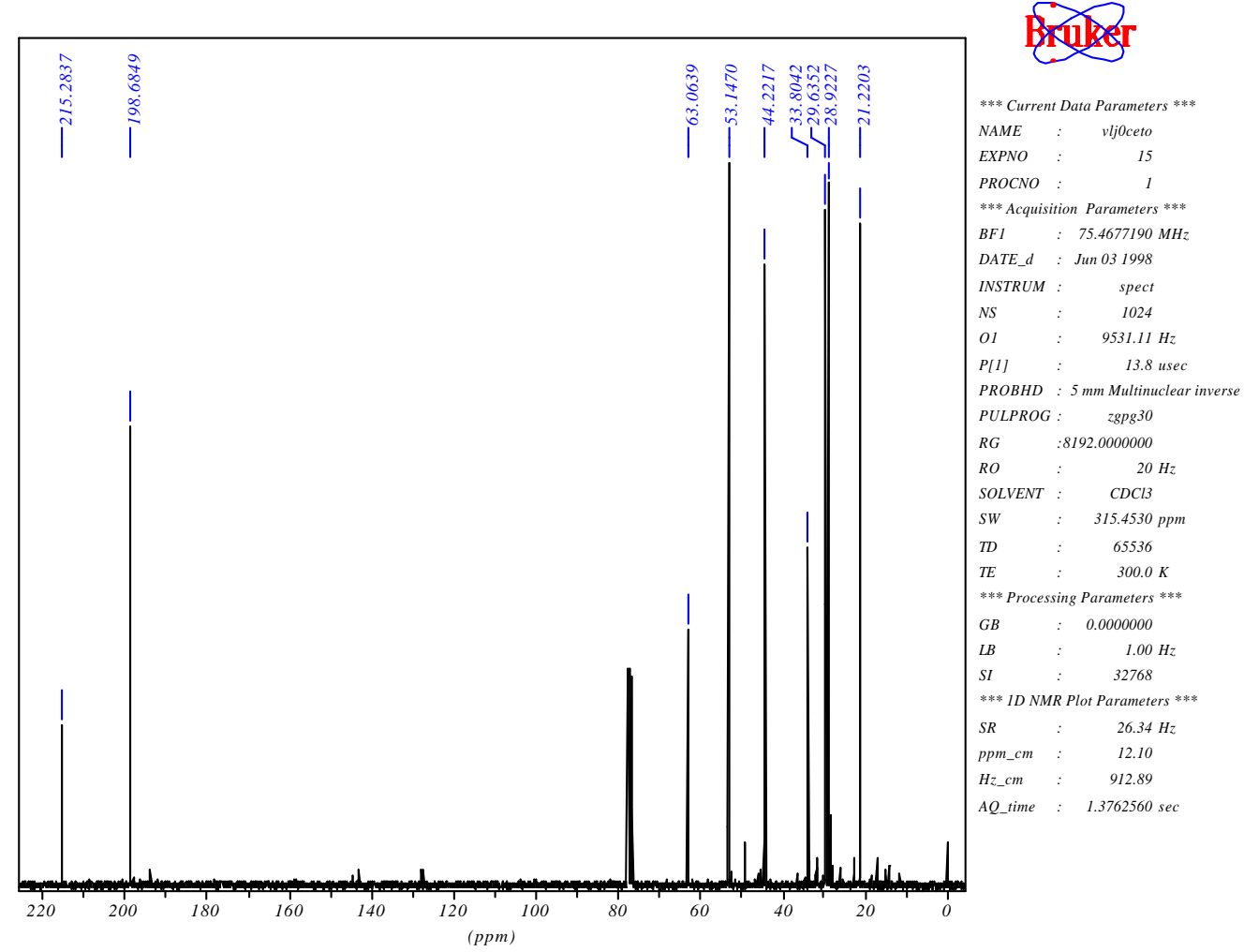

\section{Espectro de ${ }^{13}$ C-RMN (DEPT-135) do composto (3)}

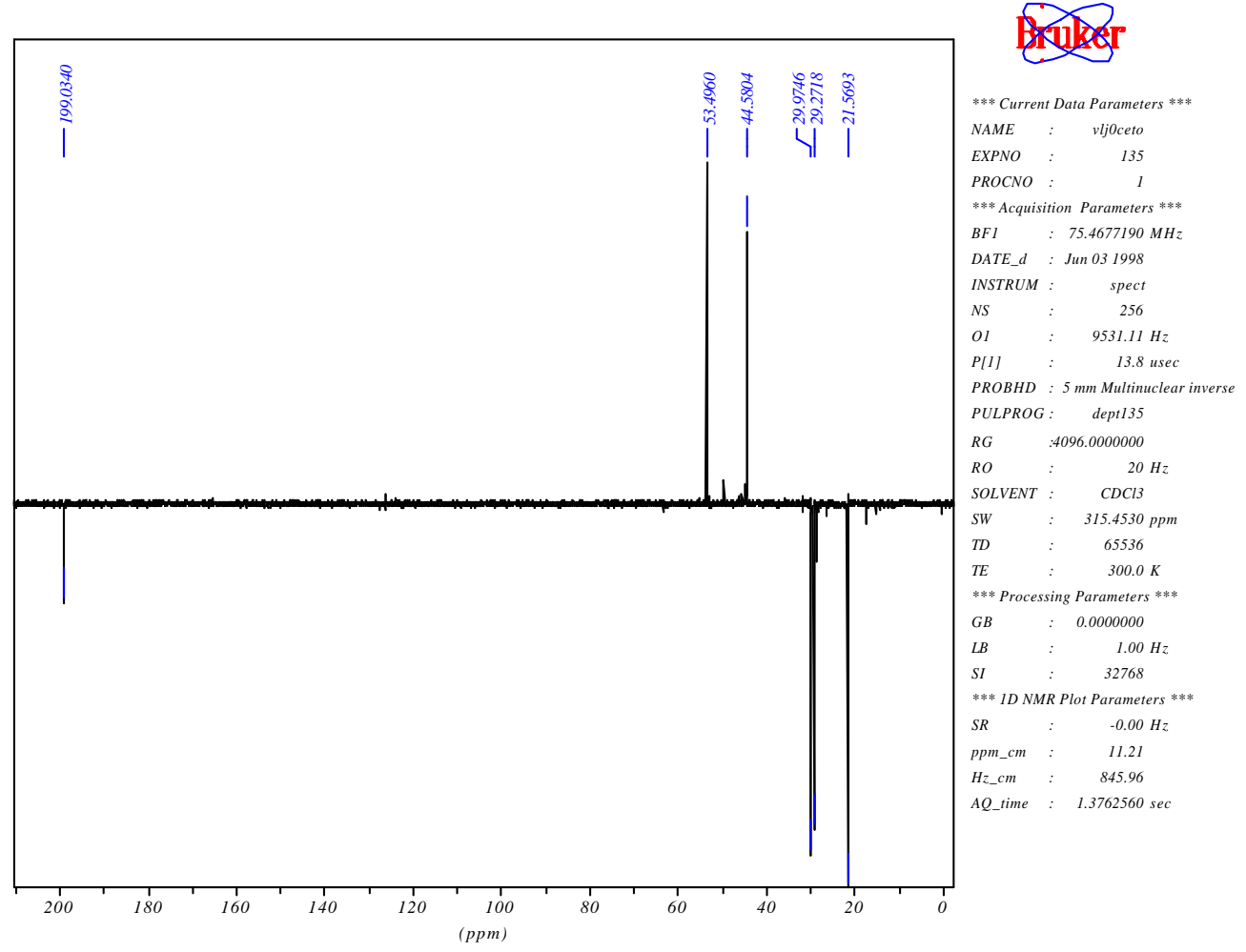




\section{Espectro HMQC (2D H/C) do composto (3)}

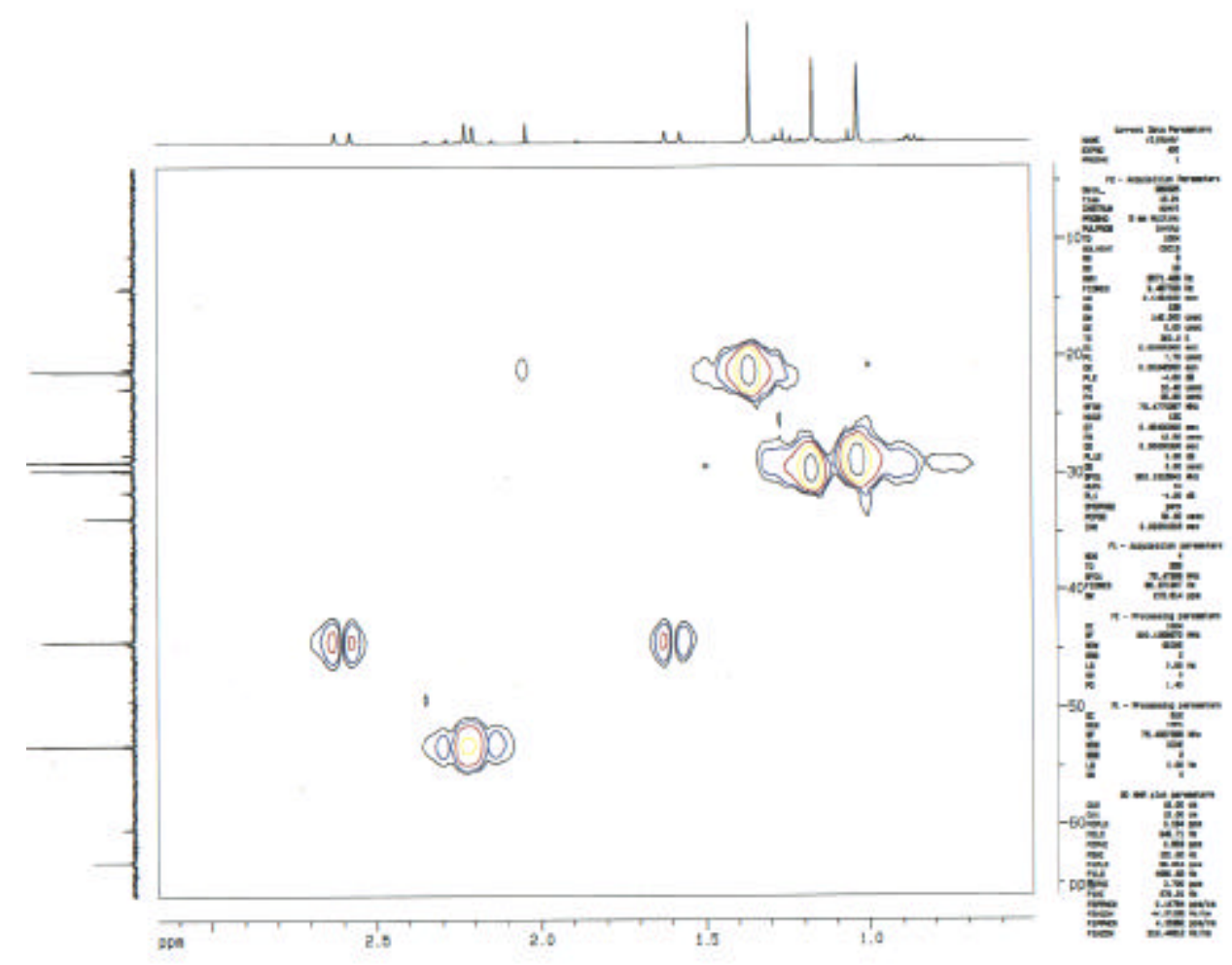

\section{Espectros NOE DIFF do composto (3)}

$>$ irradiação na metila 6

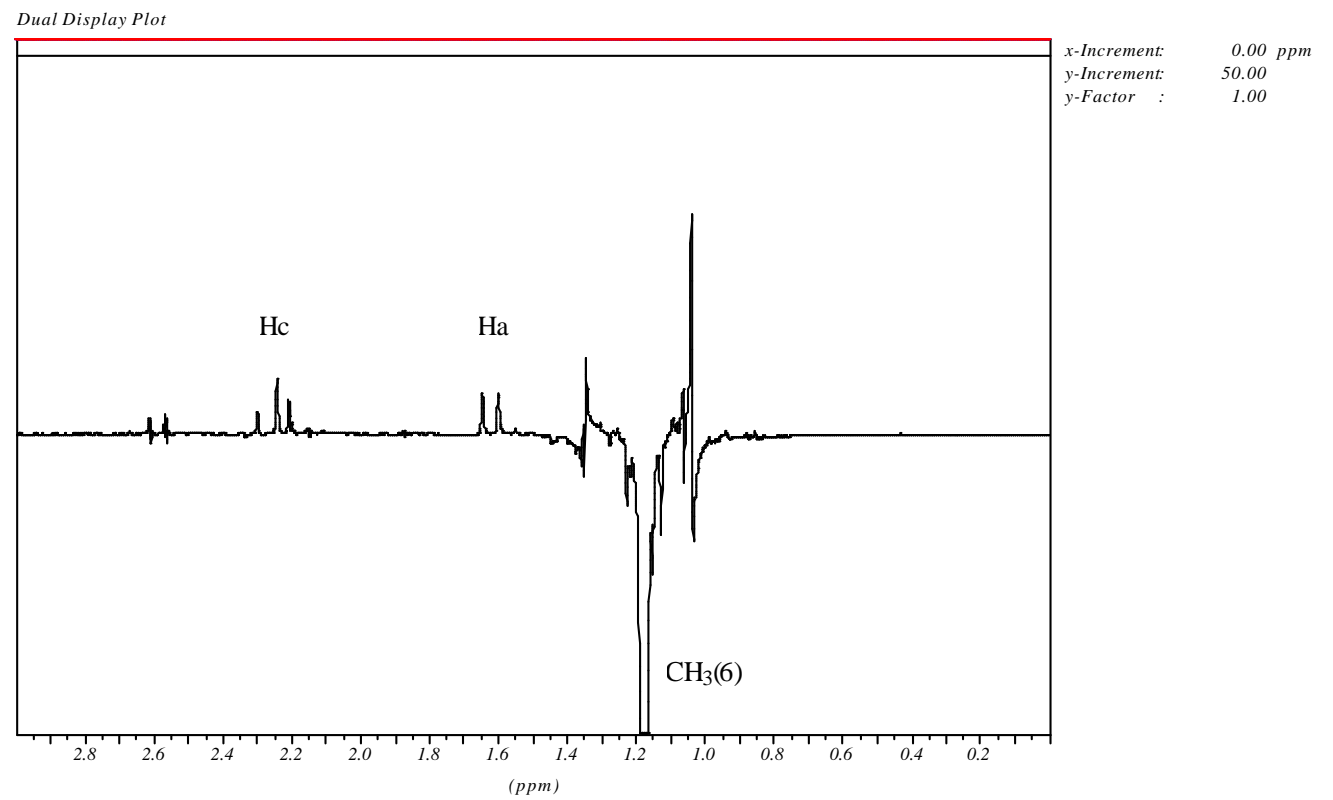

Main Trace: e: \backup $\sim 5 \backslash$ valdemarlespec $\sim 11 \backslash$ spectro\vlj0ceto $v$ ljodif $\ \backslash$ pdata $\backslash \backslash \backslash R$ Second Trace: $\backslash$ WINID \SPC\ASPX32\VLJODIF\005001.1R 
irradiação na metila 7

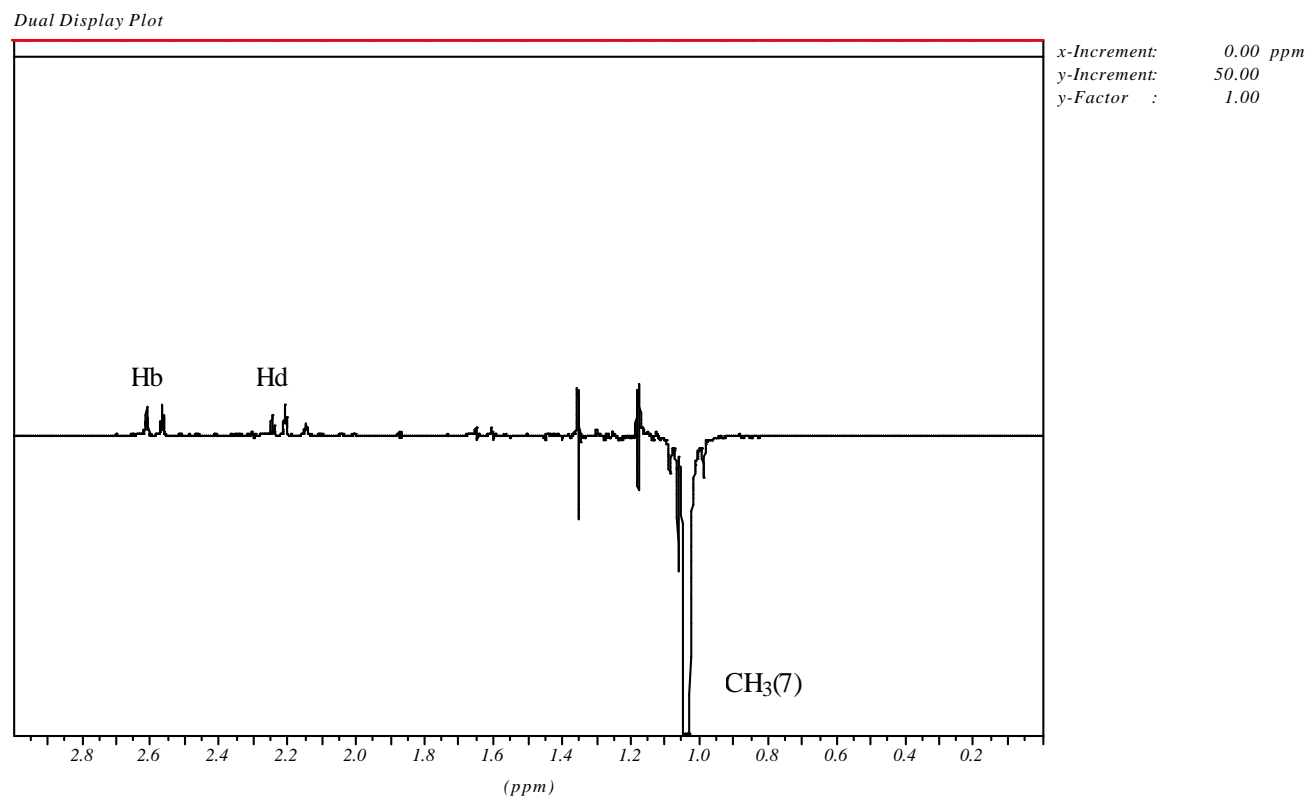

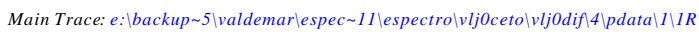
Second TraceC: $\backslash W I N I D \backslash S P C \backslash A S P X 32 \backslash V L J O D I F \backslash 005001.1 R$

irradiação na metila 8

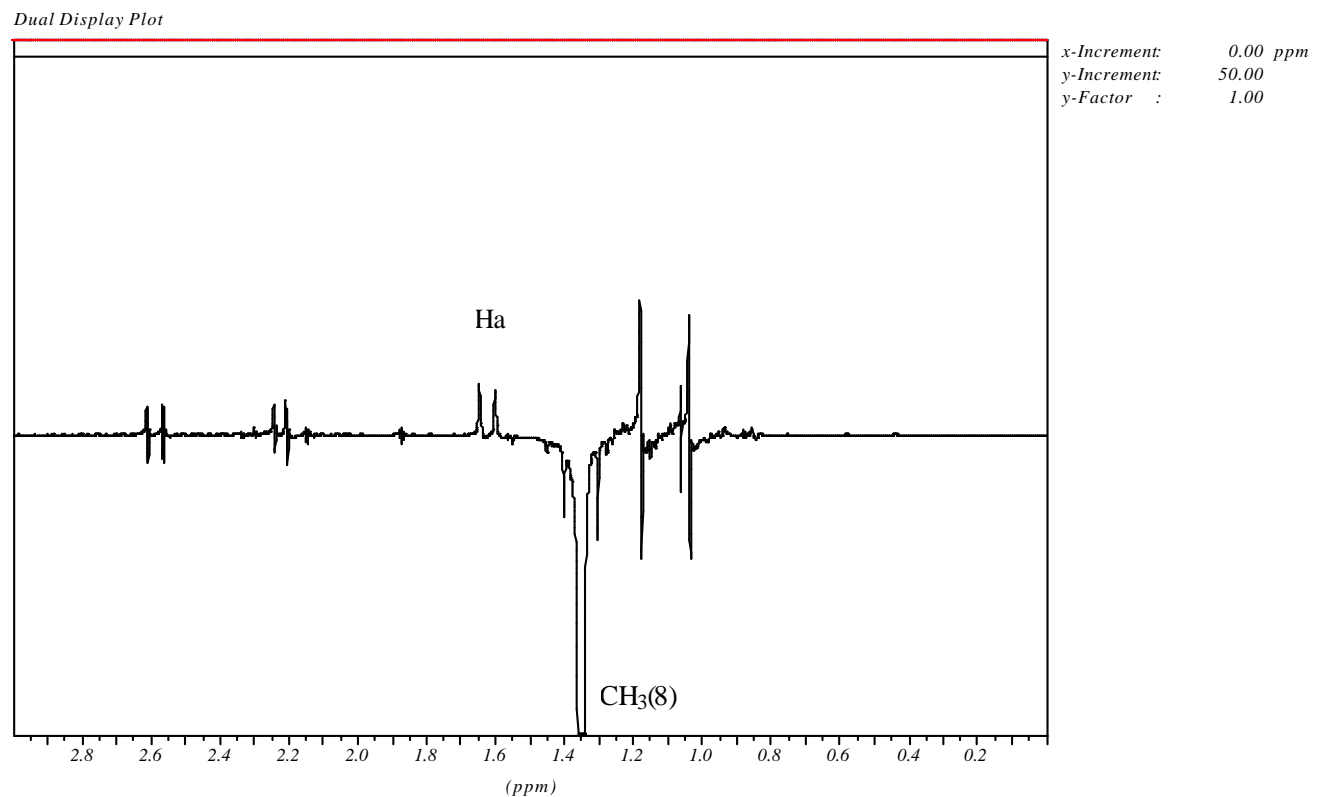

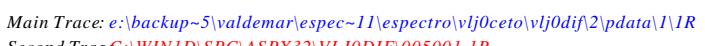

Second TracC: $\backslash W I N I D \backslash S P C \backslash A S P X 32 \backslash V L J O D I F \backslash 005001.1 R$ 
Tabela 5 - Dados espectrais de NOE DIFF do composto $3\left(\mathrm{CDCl}_{3}\right)$.

\begin{tabular}{c|c|c}
\hline$\delta(\mathbf{p p m})$ Irradiado & Atribuição & Efeito NOE Observado \\
\hline 1,35 & $\mathrm{CH}_{3}(8)$ & $\mathrm{Ha}$ \\
1,17 & $\mathrm{CH}_{3}(6)$ & $\mathrm{Ha}, \mathrm{Hc}$ \\
1,03 & $\mathrm{CH}_{3}(7)$ & $\mathrm{Hb}, \mathrm{Hd}$ \\
\hline
\end{tabular}

Tabela 6 - Dados espectrais de ${ }^{1} \mathrm{H}$ e ${ }^{13} \mathrm{C} \mathrm{RMN}$ para o $( \pm)$ - 1,4,4 - Trimetil - 2-oxociclopentano-1-carboxaldeído $\left(\mathrm{CDCl}_{3}\right)$

\begin{tabular}{|c|c|c|}
\hline $\mathrm{C}-, \mathrm{H}-$ & ${ }^{1} \mathrm{H}(300 \mathrm{MHz}) \delta(\mathrm{ppm})$ & ${ }^{13} \mathrm{C}(75 \mathrm{MHz})$ \\
\hline 1 & & 215,28 \\
\hline 2 & $\mathrm{Hc}-2,27(\mathrm{~d}, 1 \mathrm{H}, \mathrm{Jcd}=17,2 \mathrm{~Hz}) \mathrm{e} \mathrm{Hd}-2,17(\mathrm{dd}, 1 \mathrm{H}, \mathrm{Jdc}=17,2 \mathrm{e}$ & 53,14 \\
$\mathrm{Jdb}=1 \mathrm{~Hz})$ & 33,8 \\
\hline 3 & & 44,22 \\
\hline 4 & $\mathrm{Hb}-2,59(\mathrm{dd}, 1 \mathrm{H}, \mathrm{Jba}=13,7 \mathrm{e} \mathrm{Jbd}=1 \mathrm{~Hz}) \mathrm{e} \mathrm{Ha}-1,61(\mathrm{~d}, 1 \mathrm{H}$, & 63,06 \\
\hline 5 & $\mathrm{Jab}=13,7 \mathrm{~Hz})$ & 29,63 \\
\hline 6 & $1,17(\mathrm{~s}, 3 \mathrm{H})$ & 28,92 \\
\hline 7 & $1,03(\mathrm{~s}, 3 \mathrm{H})$ & 21,22 \\
\hline 8 & $1,35(\mathrm{~s}, 3 \mathrm{H})$ & 198,68 \\
\hline 9 & $9,48(\mathrm{~s}, 1 \mathrm{H})$ & \\
\hline
\end{tabular}




\section{Composto (4)}<smiles>[CH][C@@H]1C(C)(C)[C@@H](C)C(=O)[C@@]1(C)C(=O)OC</smiles>

Espectro de ${ }^{1}$ H-RMN do composto (4)

\section{4}

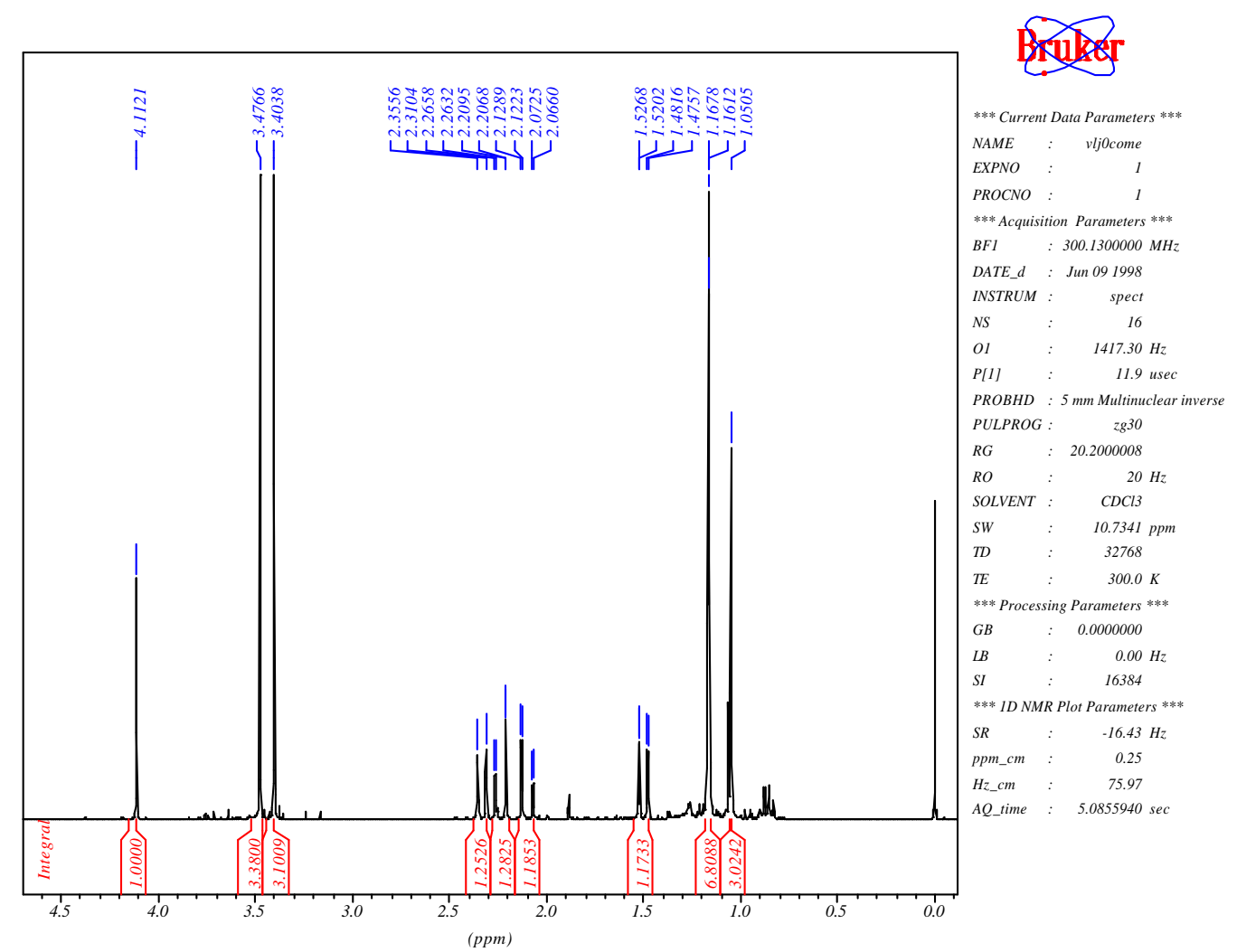

Tabela 7 - Dados espectrais de ${ }^{1} \mathrm{H}$ RMN do composto $4\left(\mathrm{CDCl}_{3}\right)$.

\begin{tabular}{c|c|c|c}
\hline$\delta$ (ppm) & Atribuição & sinal & J (Hz) \\
\hline 4,11 & 9 & $\mathrm{~s}$ & \\
3,47 & 11 & $\mathrm{~s}$ & \\
3,40 & 10 & $\mathrm{~s}$ & \\
2,33 & $\mathrm{Hb}$ & $\mathrm{dd}$ & $\mathrm{Jba}=13,5 \mathrm{e} \mathrm{Jbd}=0,8 \mathrm{~Hz}$ \\
2,23 & $\mathrm{Hd}$ & $\mathrm{dd}$ & $\mathrm{Jdc}=16,9, \mathrm{Jdb}=0,8 \mathrm{~Hz}$ \\
2,10 & $\mathrm{Hc}$ & $\mathrm{dd}$ & $\mathrm{Jcd}=16,9 \mathrm{e} \mathrm{Jca}=2 \mathrm{~Hz}$ \\
1,50 & $\mathrm{Ha}$ & $\mathrm{dd}$ & $\mathrm{Jab}=13,5 \mathrm{e} \mathrm{Jac}=2 \mathrm{~Hz}$ \\
1,17 & $\mathrm{CH}_{3}(7)$ & $\mathrm{s}$ & \\
1,16 & $\mathrm{CH}_{3}(8)$ & $\mathrm{s}$ & \\
1,05 & $\mathrm{CH}_{3}(6)$ & $\mathrm{s}$ & \\
\hline
\end{tabular}


Espectro de ${ }^{13} \mathrm{C}$-RMN (BB) do composto (4)

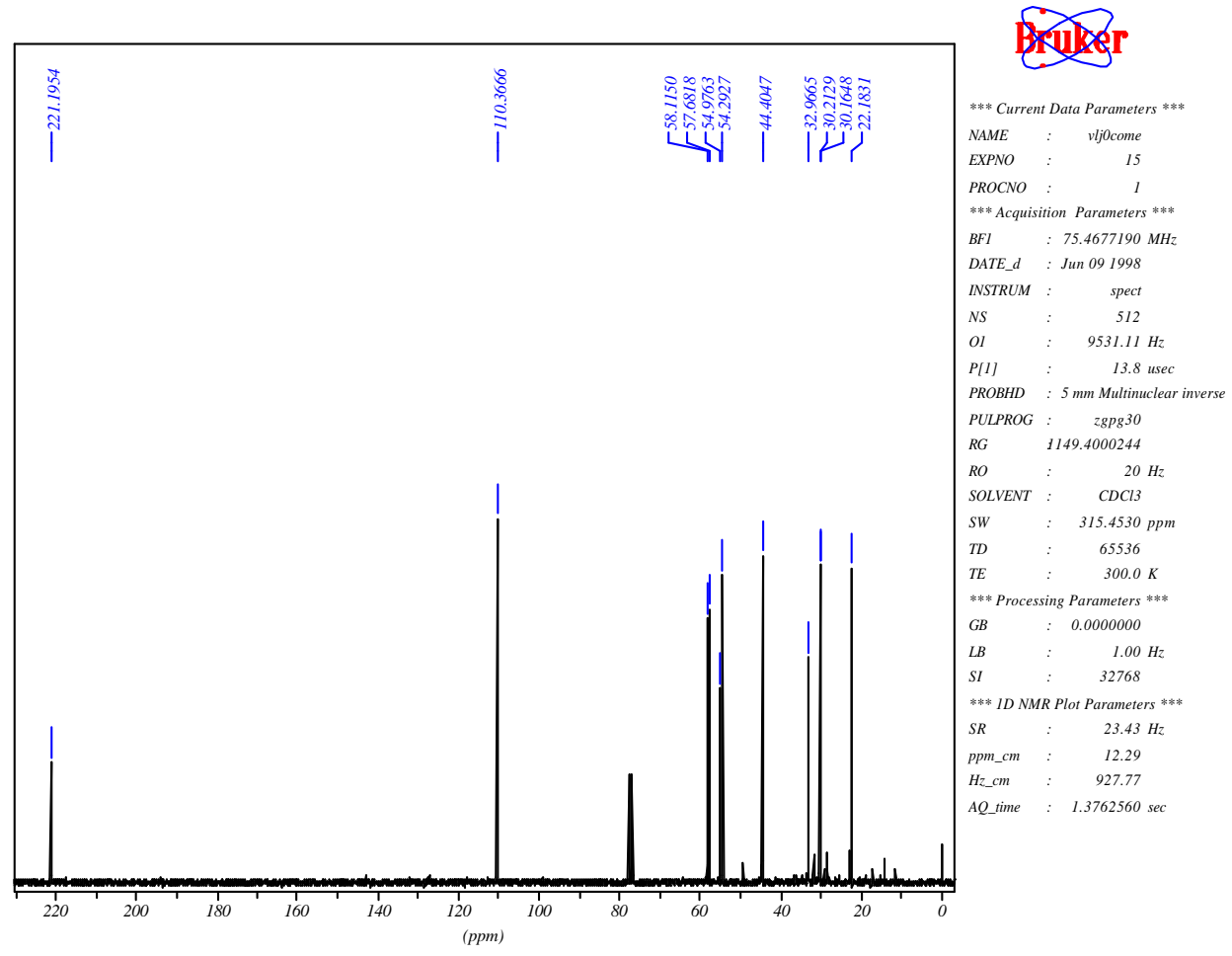

\section{Espectro de ${ }^{13}$ C-RMN (DEPT-135) do composto (4)}

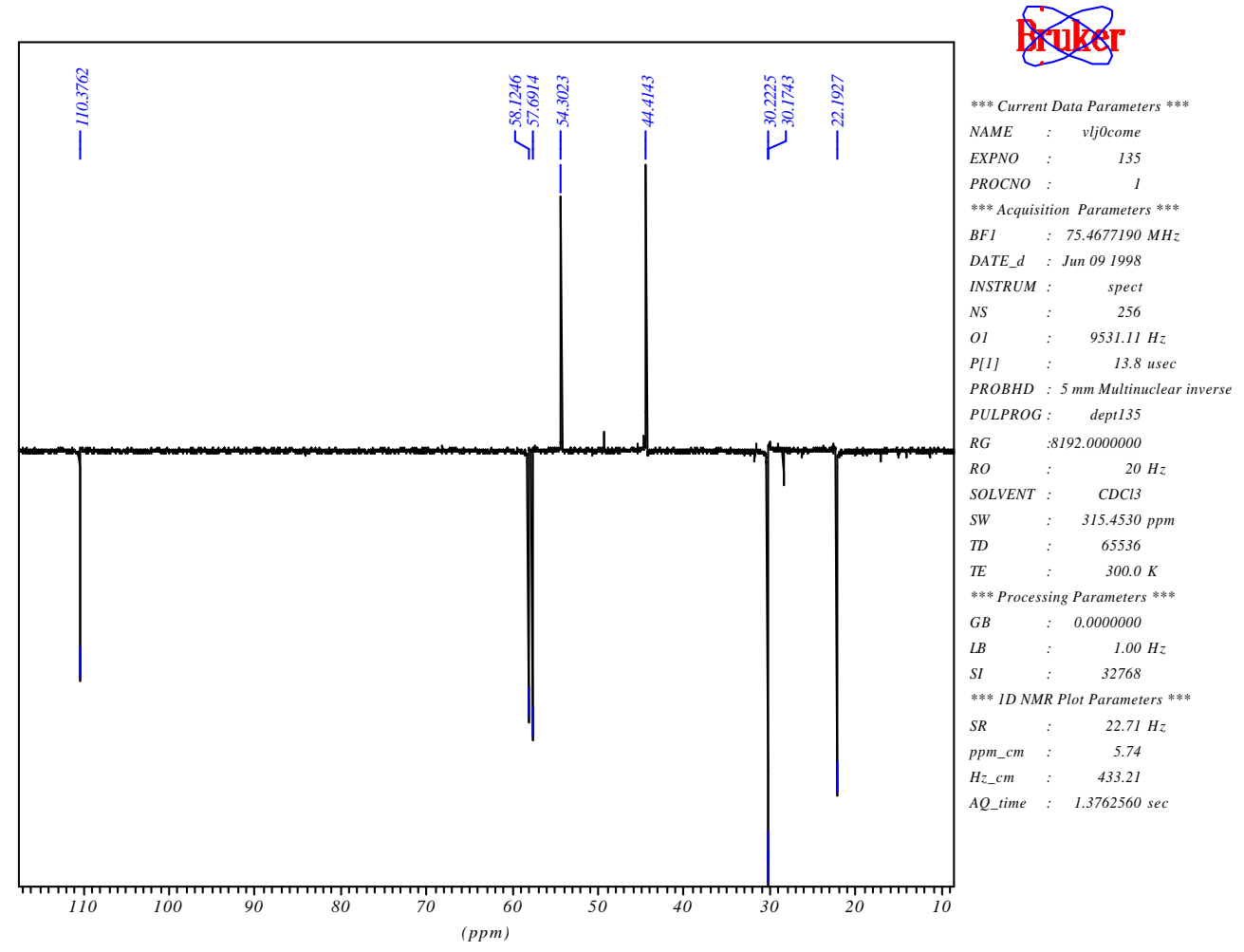




\section{Espectro HMQC (2D H/C) do composto (4)}
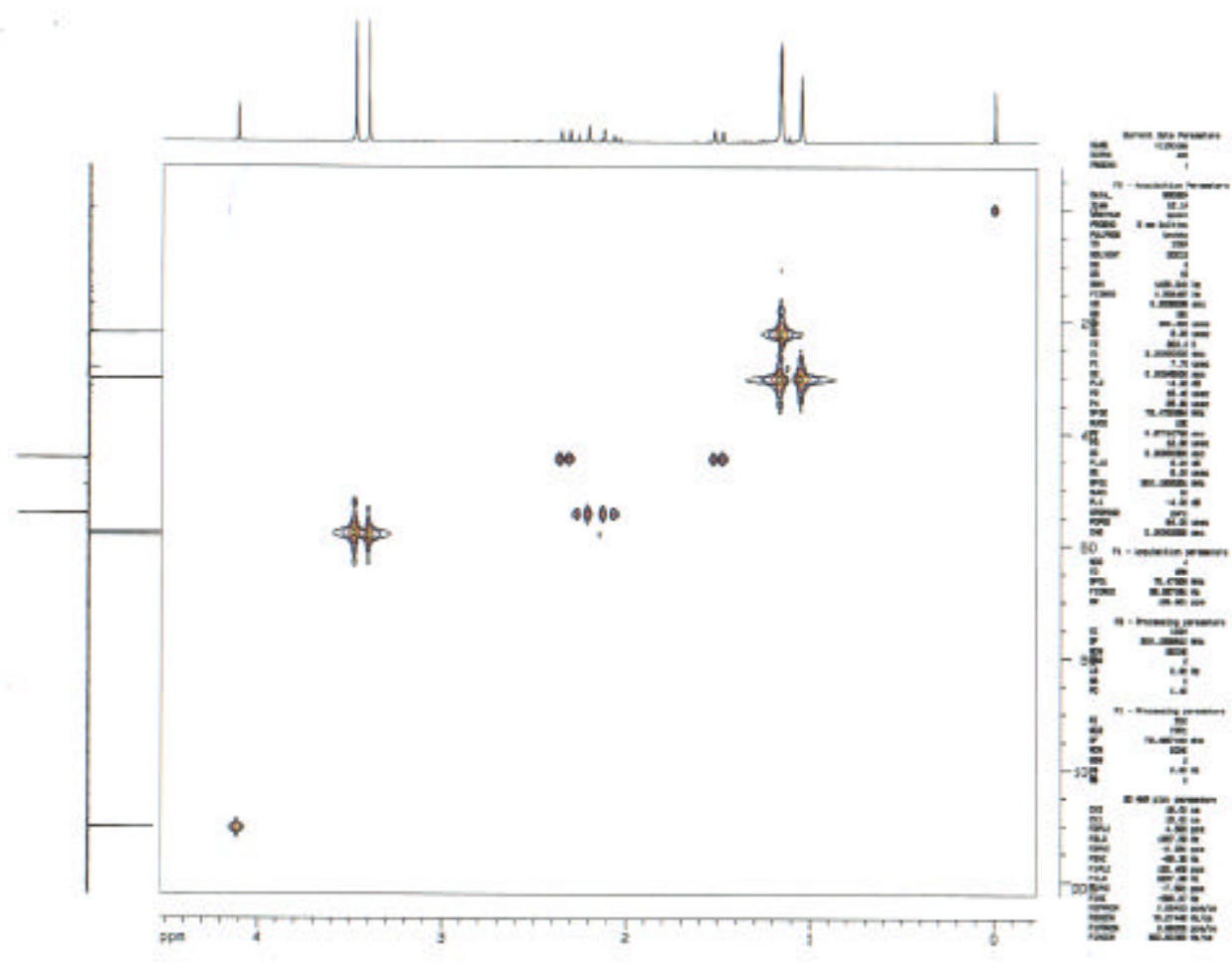

Espectro Jres do composto (4)
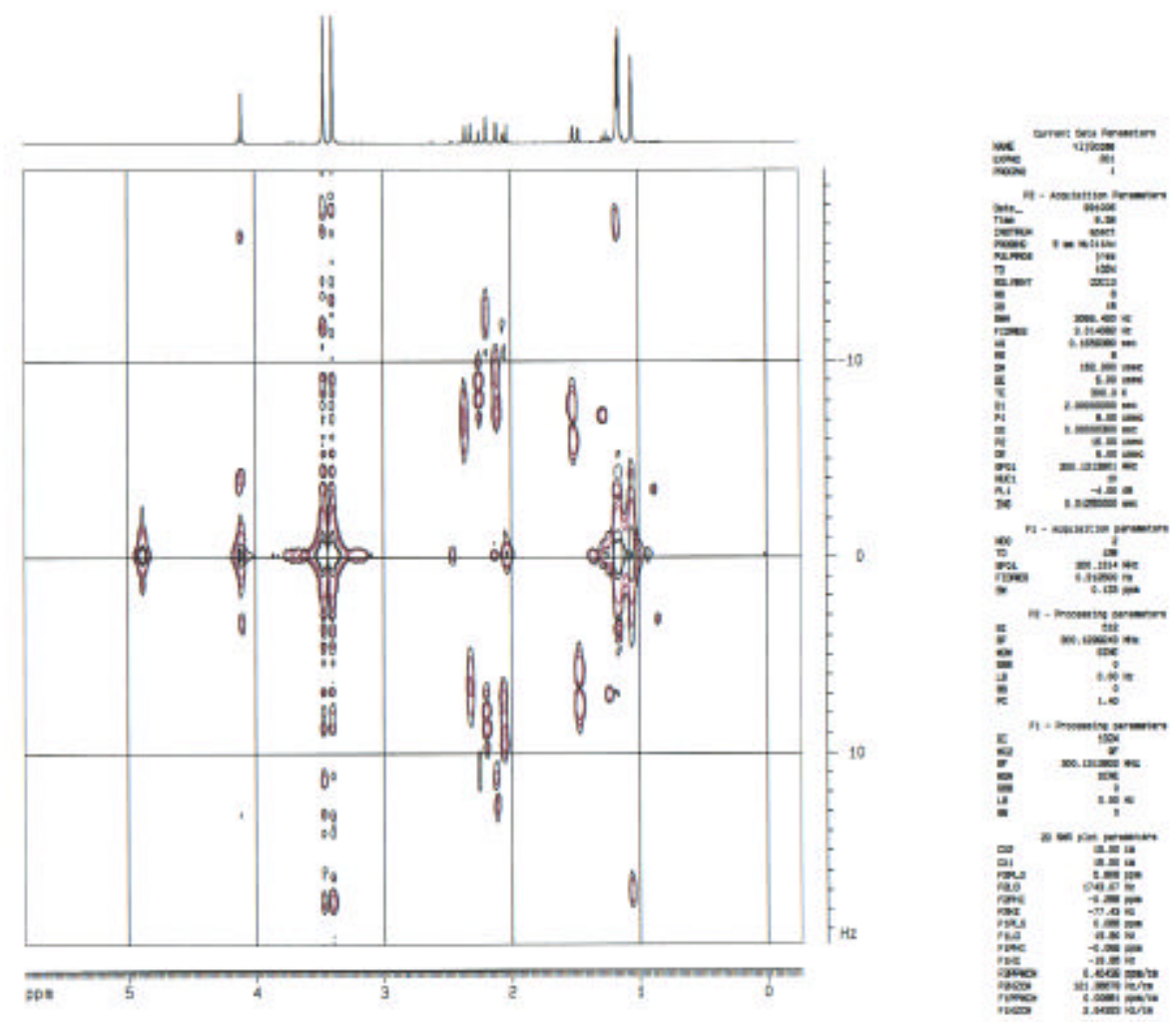


\section{Espectros NOE DIFF do composto (4)}

$>$ irradiação na metila 6

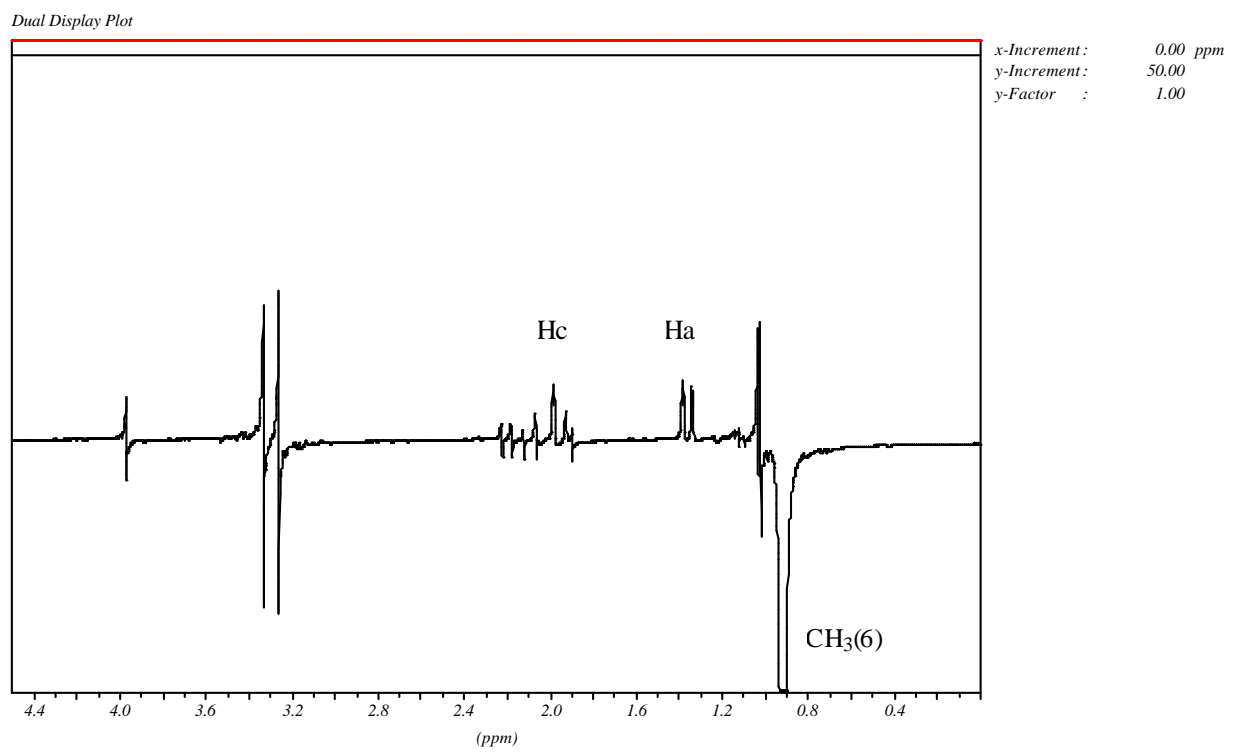

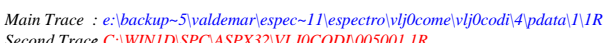

Second Trace C:IWINIDISPCASPX321VLJOCODN005001.1R

irradiação na metila 7

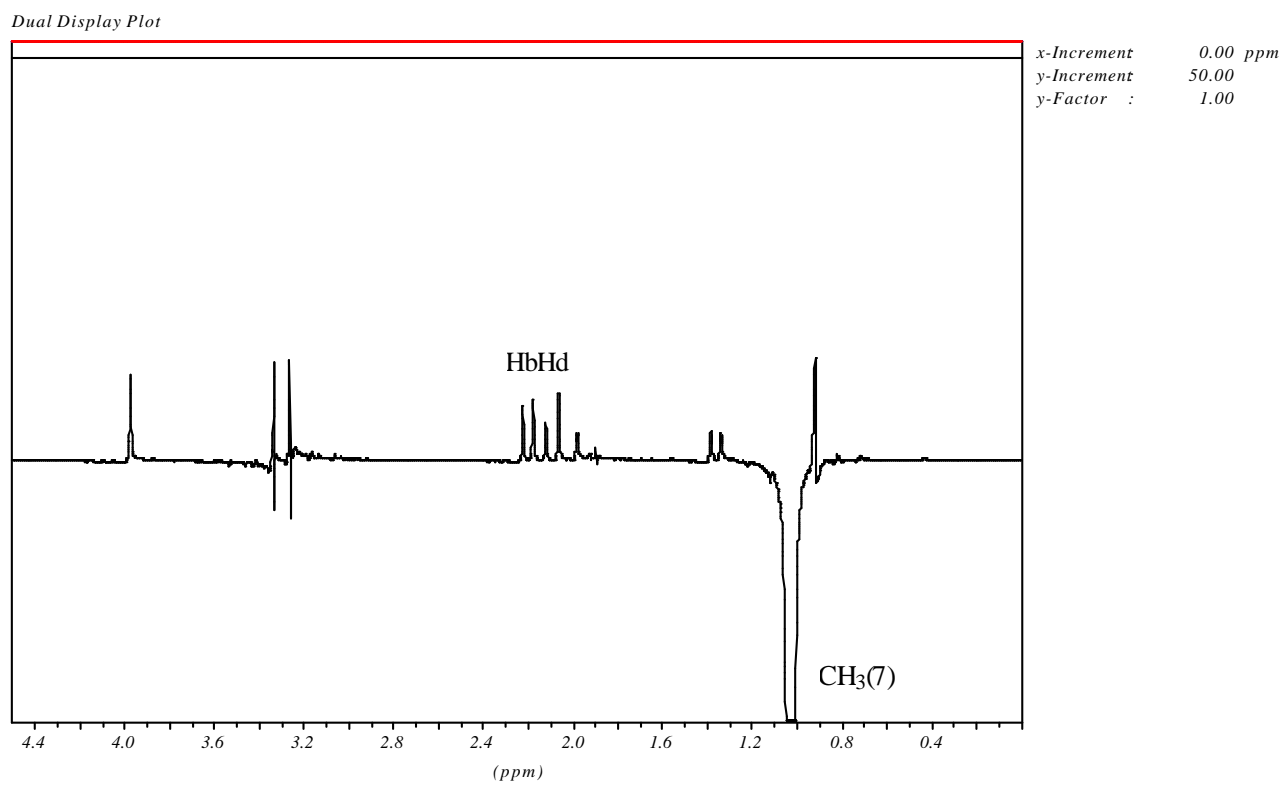

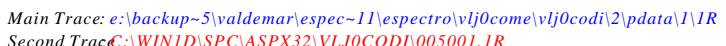


irradiação na metila 8

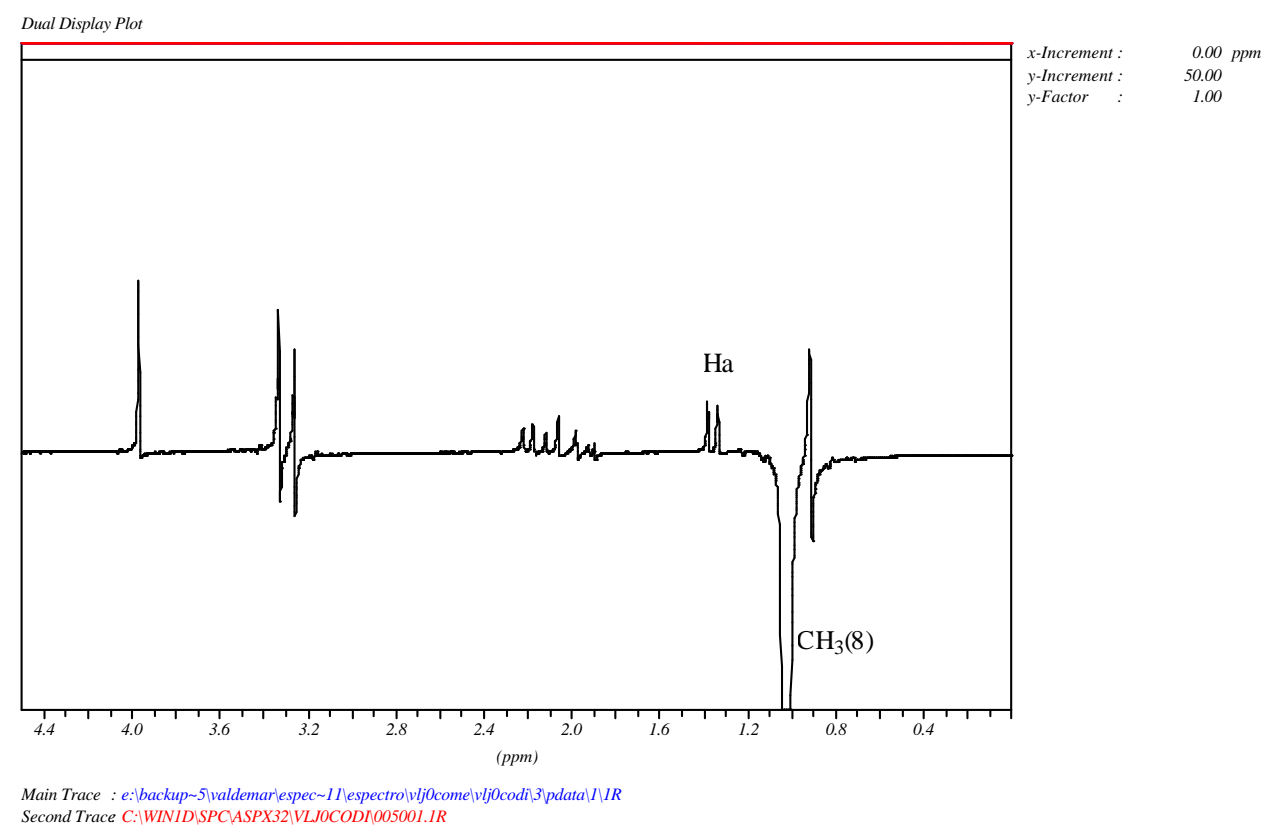

Tabela 8 - Dados espectrais de NOE DIFF do composto $4\left(\mathrm{CDCl}_{3}\right)$.

\begin{tabular}{c|c|c}
\hline$\delta(\mathbf{p p m})$ Irradiado & Atribuição & Efeito NOE Observado \\
\hline 1,17 & $\mathrm{CH}_{3}(7)$ & $\mathrm{Hb}, \mathrm{Hd}$ \\
1,16 & $\mathrm{CH}_{3}(8)$ & $\mathrm{Ha}$ \\
1,05 & $\mathrm{CH}_{3}(6)$ & $\mathrm{Ha}, \mathrm{Hc}$ \\
\hline
\end{tabular}

Tabela 9 - Dados espectrais de ${ }^{1} \mathrm{H}$ e ${ }^{13} \mathrm{C}$ RMN para o $( \pm)$ - 2 - Dimetoximetil -2,4,4 trimetil - ciclopentanona $\left(\mathrm{CDCl}_{3}\right)$

\begin{tabular}{|c|c|c|}
\hline$\overline{C-}, H_{-}$ & ${ }^{1} \mathrm{H}(300 \mathrm{MHz}) \delta(\mathrm{ppm})$ & ${ }^{13} \mathrm{C}(75 \mathrm{MHz})$ \\
\hline 1 & & 221,19 \\
\hline 2 & $\begin{array}{c}\mathrm{Hd}-2,23(\mathrm{dd}, 1 \mathrm{H}, \mathrm{Jdc}=16,9, \mathrm{Jdb}=0,8 \mathrm{~Hz}) \text { e } \mathrm{Hc}-2,10(\mathrm{dd}, 1 \mathrm{H}, \\
\mathrm{Jcd}=16,9 \text { e Jca }=2 \mathrm{~Hz})\end{array}$ & 54,29 \\
\hline 3 & & 32,96 \\
\hline 4 & $\begin{array}{c}\mathrm{Hb}-2,33(\mathrm{dd}, 1 \mathrm{H}, \mathrm{Jba}=13,5 \text { e } \mathrm{Jbd}=0,8 \mathrm{~Hz}) \text { e } \mathrm{Ha}-1,50(\mathrm{dd}, 1 \mathrm{H}, \\
\mathrm{Jab}=13,5 \text { e } \mathrm{Jac}=2 \mathrm{~Hz})\end{array}$ & 44,40 \\
\hline 5 & & 54,97 \\
\hline 6 & $1,05(\mathrm{~s}, 3 \mathrm{H})$ & 30,16 \\
\hline 7 & $1,17(\mathrm{~s}, 3 \mathrm{H})$ & 30,21 \\
\hline 8 & $1,16(\mathrm{~s}, 3 \mathrm{H})$ & 22,18 \\
\hline 9 & $4,11(\mathrm{~s}, 1 \mathrm{H})$ & 110,36 \\
\hline 10 & $3,40(\mathrm{~s}, 3 \mathrm{H})$ & 58,11 \\
\hline 11 & $3,47(\mathrm{~s}, 3 \mathrm{H})$ & 57,68 \\
\hline
\end{tabular}




\section{Composto (5a)}

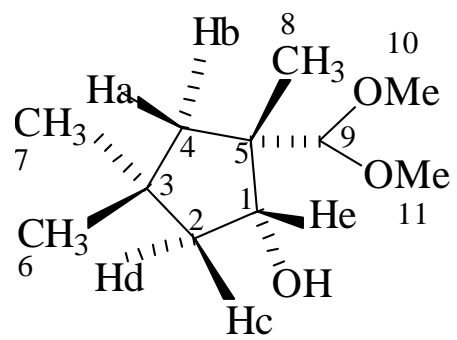

\section{Espectro de ${ }^{1} \mathrm{H}-\mathrm{RMN}$ do composto (5a)}

$5 a$

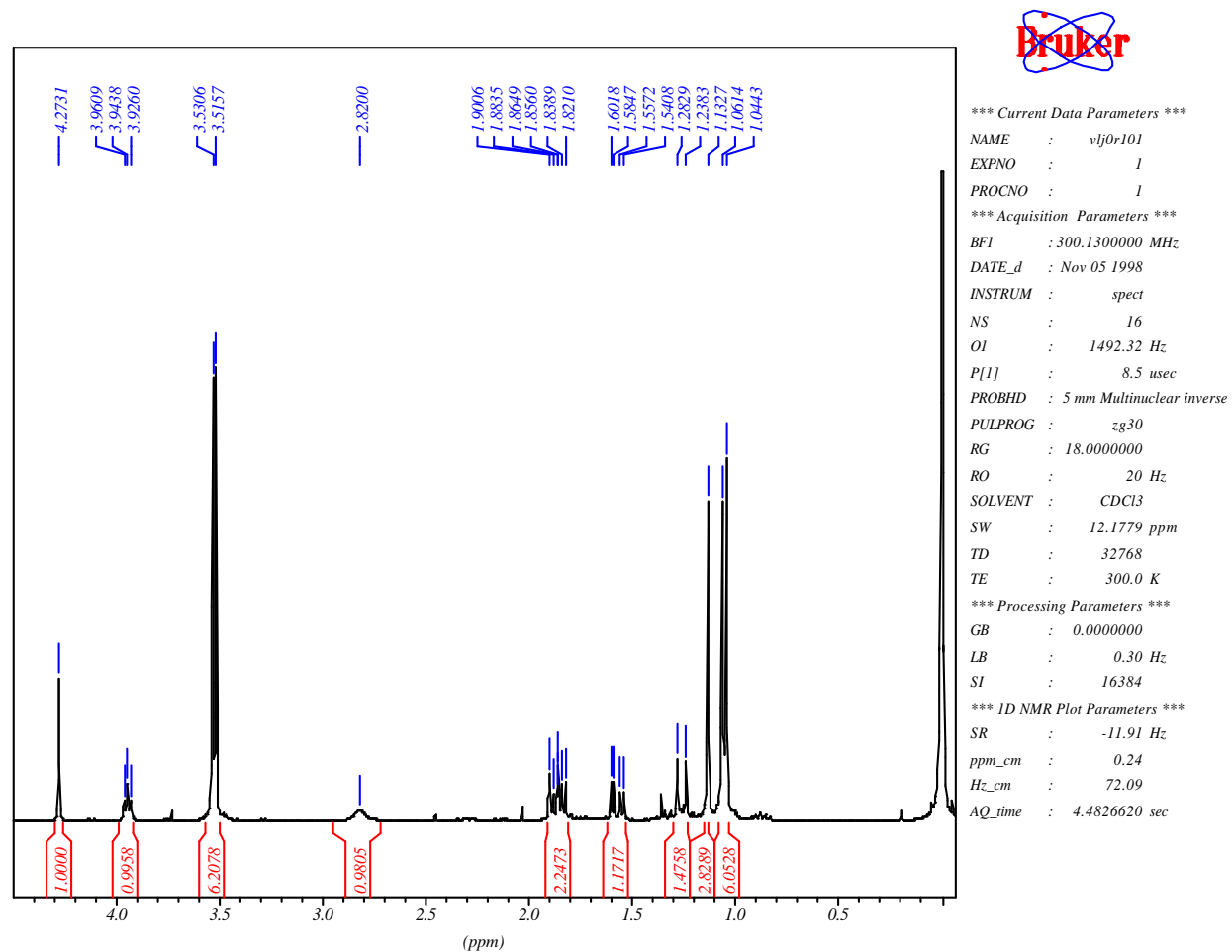

Tabela 10 - Dados espectrais de ${ }^{1} \mathrm{H}$ RMN do composto $\mathbf{5 a}\left(\mathrm{CDCl}_{3}\right)$.

\begin{tabular}{c|c|c|c}
\hline$\delta(\mathbf{p p m})$ & Atribuição & sinal & J (Hz) \\
\hline 4,27 & 9 & $\mathrm{~s}$ & \\
3,94 & $\mathrm{He}$ & $\mathrm{t}$ & $\mathrm{Jec}=\mathrm{Jed}=5 \mathrm{~Hz}$ \\
3,53 & 11 & $\mathrm{~s}$ & \\
3,51 & 10 & $\mathrm{~s}$ & \\
2,82 & $\mathrm{OH}$ & $\mathrm{sl}$ & \\
1,89 & $\mathrm{Hb}$ & $\mathrm{d}$ & $\mathrm{Jba}=13,3 \mathrm{~Hz}$ \\
1,84 & $\mathrm{Hc}$ & $\mathrm{ddd}$ & $\mathrm{Jcb}=13,2 ; \mathrm{Jce}=5 \mathrm{e} \mathrm{Jca}=1,3 \mathrm{~Hz}$ \\
1,57 & $\mathrm{Hd}$ & $\mathrm{dd}$ & $\mathrm{Jdc}=13,2 \mathrm{e} \mathrm{Jde}=5 \mathrm{~Hz}$ \\
1,26 & $\mathrm{Ha}$ & $\mathrm{dd}$ & $\mathrm{Jab}=13,3 \mathrm{e} \mathrm{Jac}=1,3 \mathrm{~Hz}$ \\
1,13 & $\mathrm{CH}_{3}(7)$ & $\mathrm{s}$ & \\
1,06 & $\mathrm{CH}_{3}(6)$ & $\mathrm{s}$ & \\
1,04 & $\mathrm{CH}_{3}(8)$ & $\mathrm{s}$ & \\
\hline
\end{tabular}




\section{Espectro de ${ }^{13}$ C-RMN (BB) do composto (5a)}

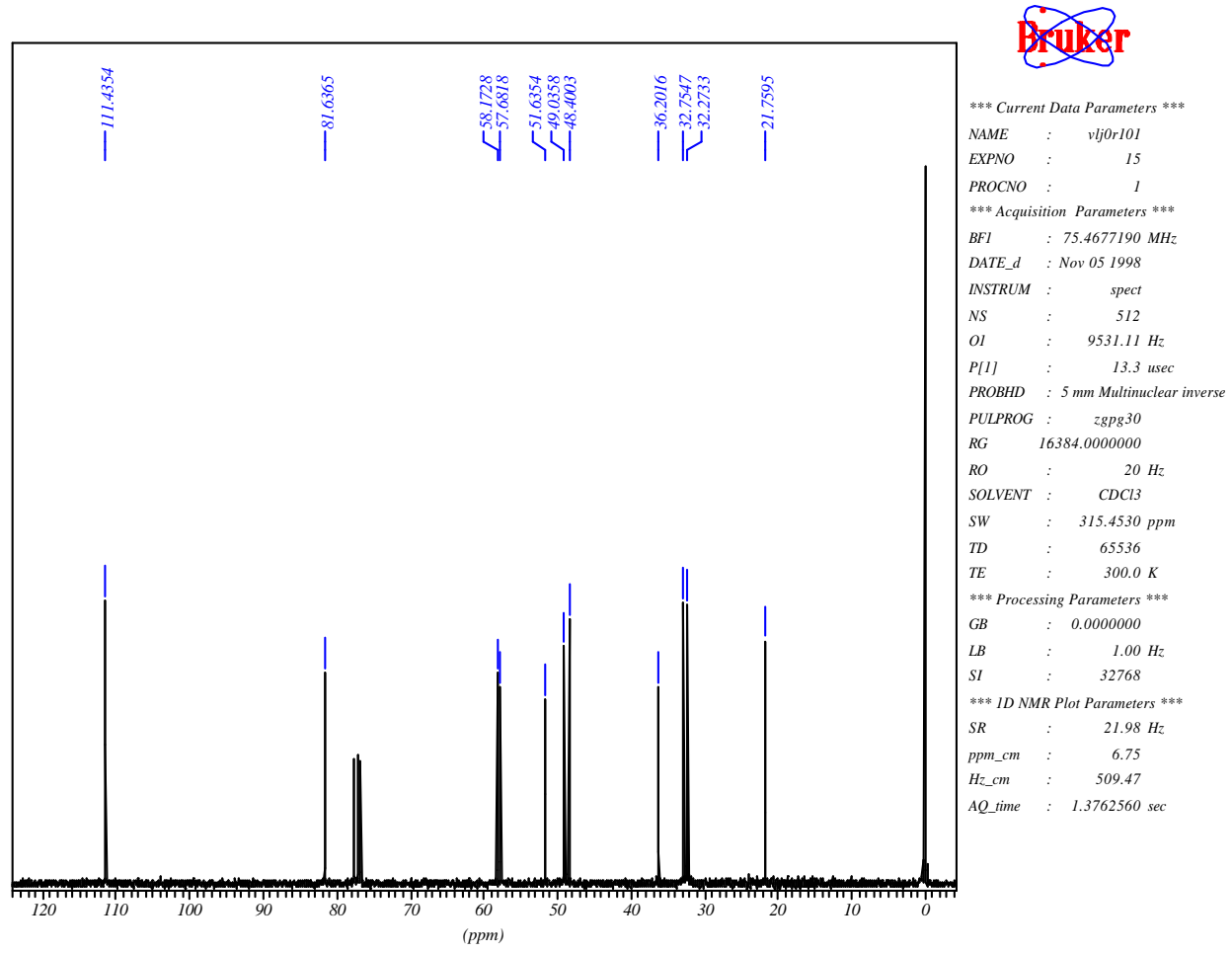

Espectro de ${ }^{13}$ C-RMN (DEPT-135) do composto (5a)

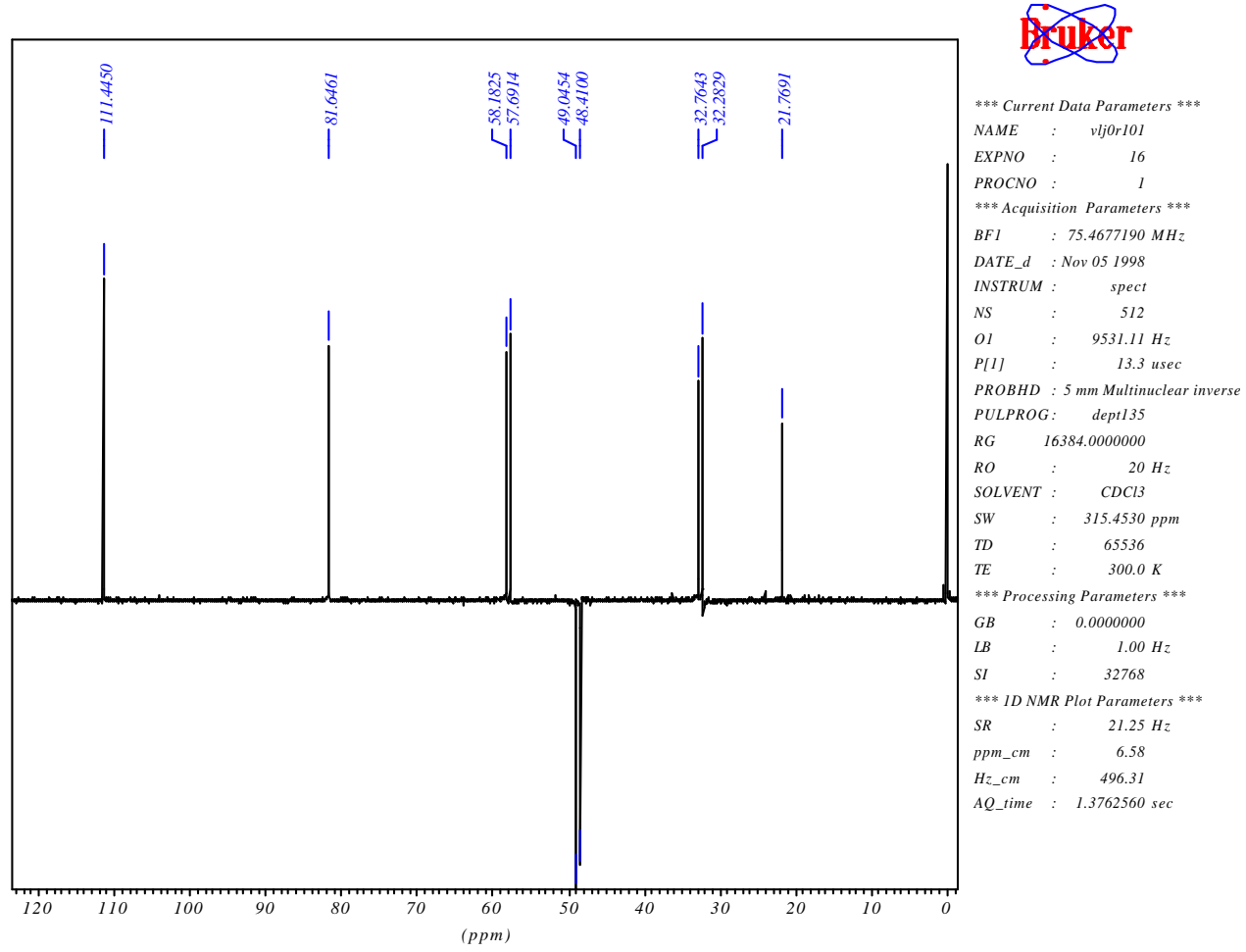


Espectro HMQC (2D H/C) do composto (5a)
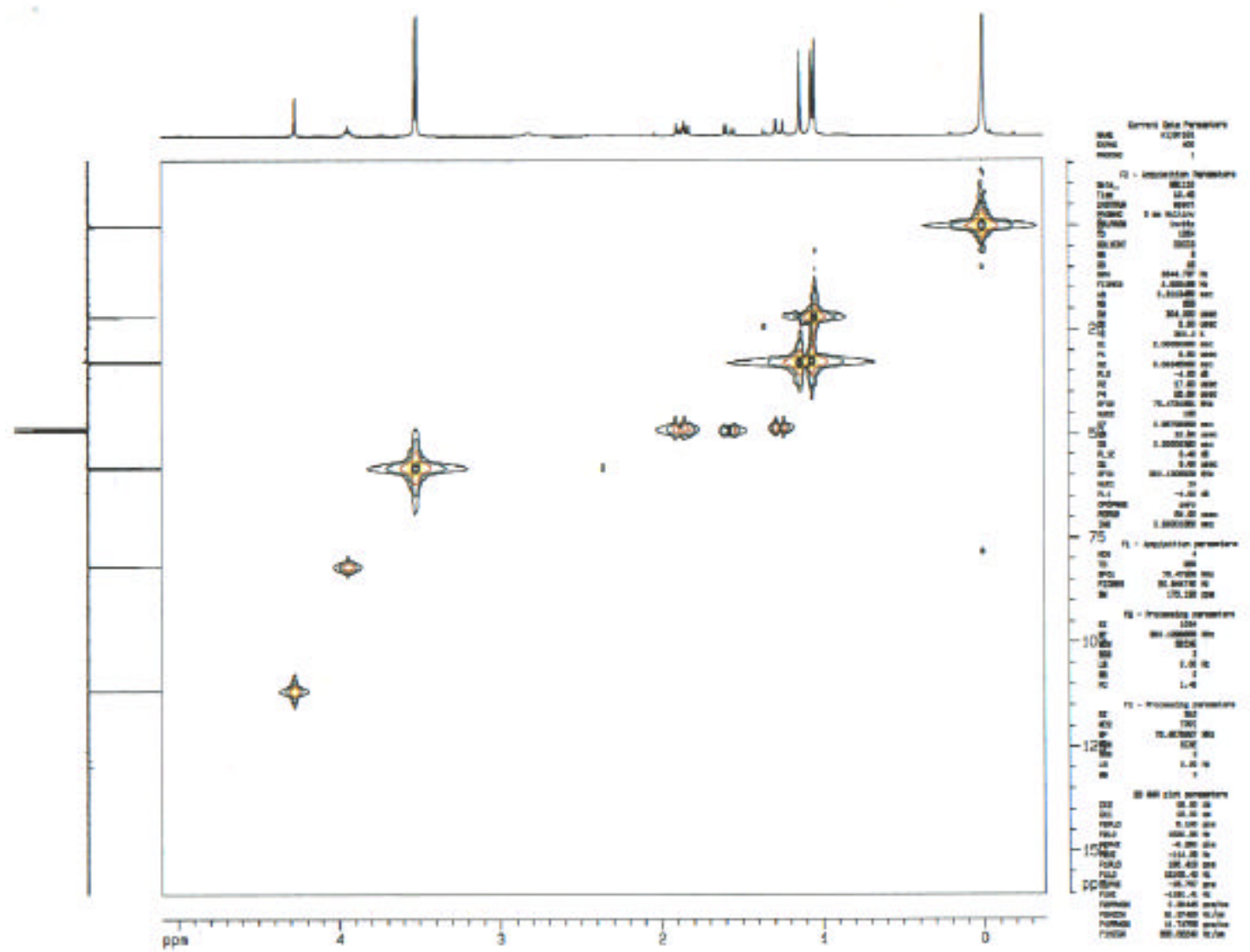

Espectro Jres do composto (5a)
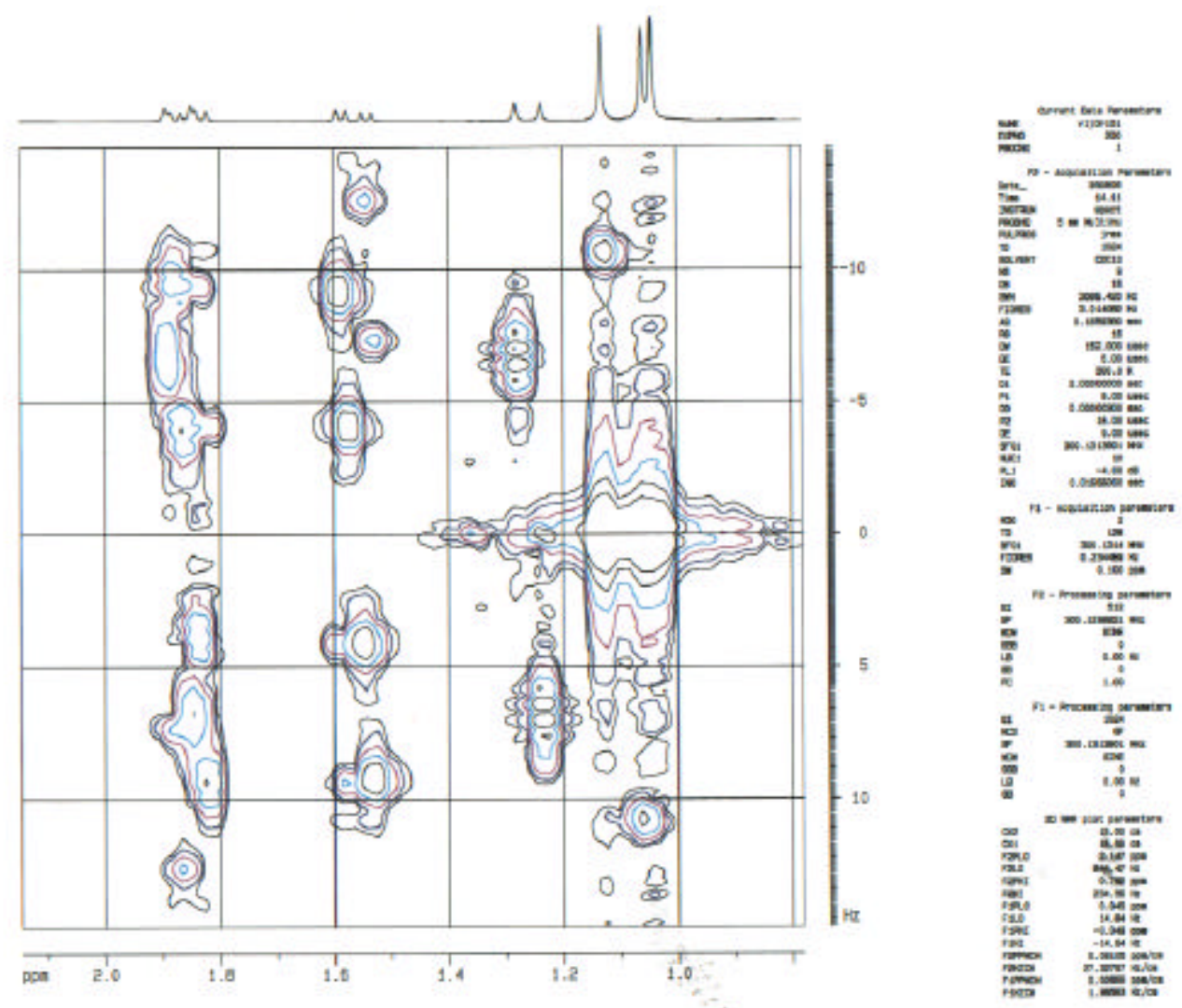


\section{Espectros NOE DIFF do composto (5a)}

$>$ irradiação na metila 6

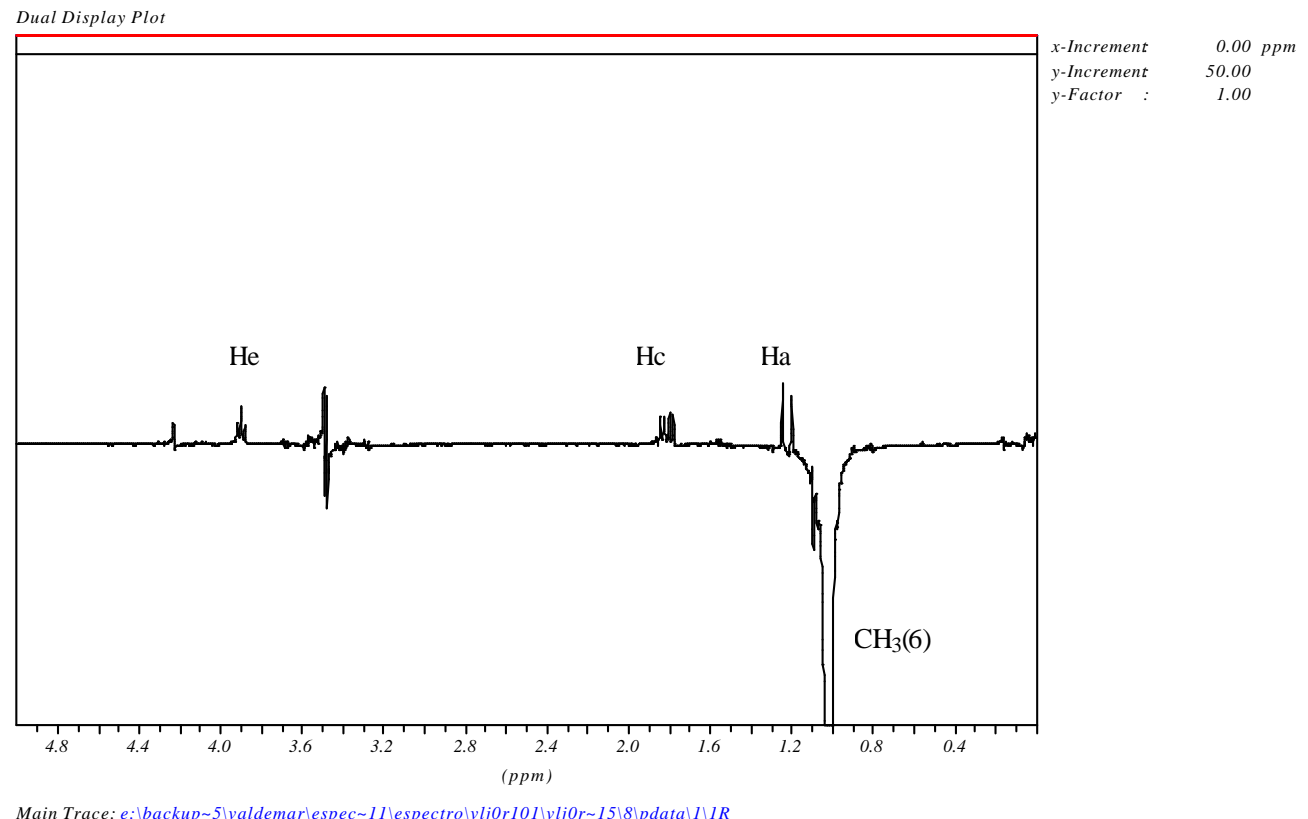

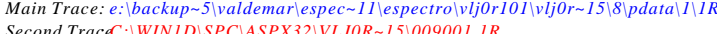

$>$ irradiação na metila 7

Dual Display Plot

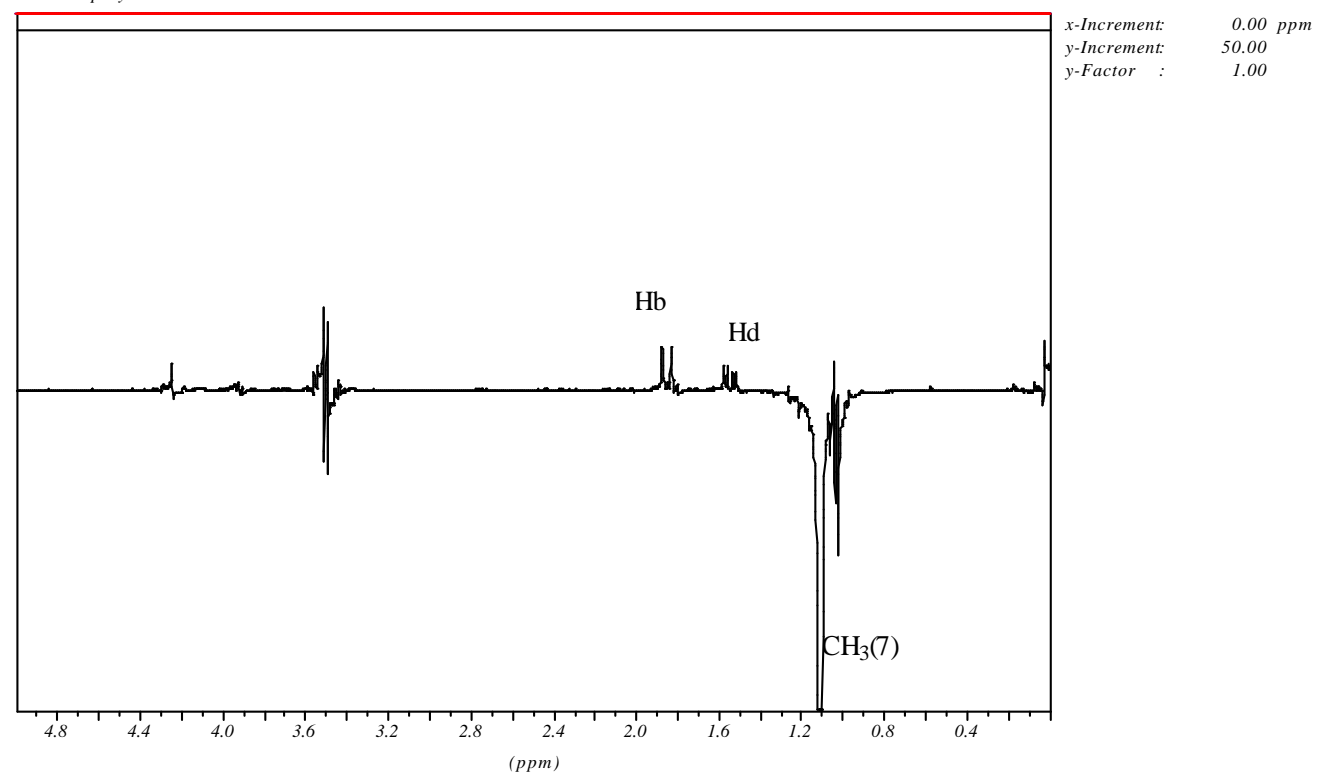

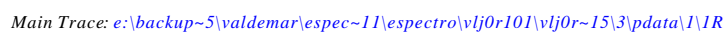

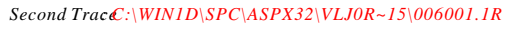


irradiação na metila 8

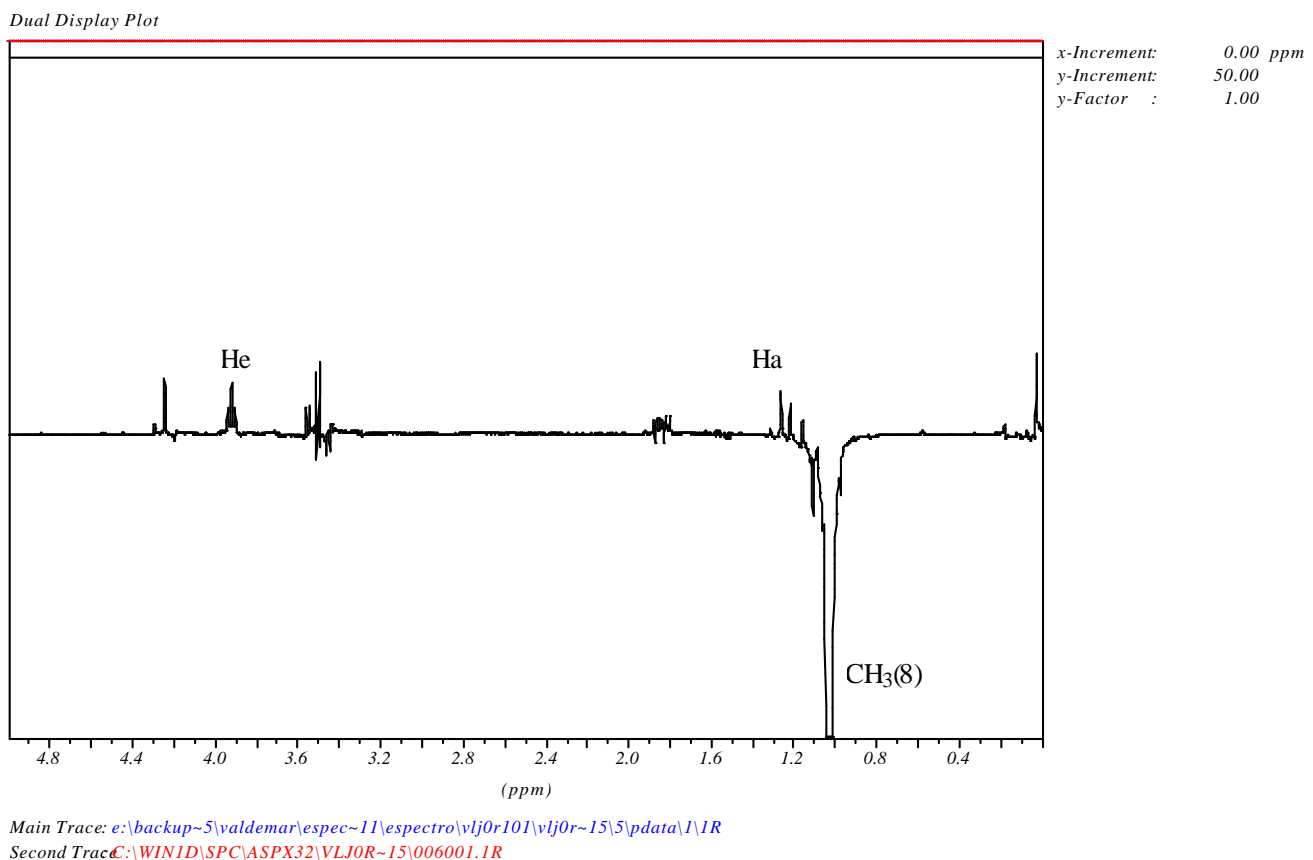

Tabela 11 - Dados espectrais de ${ }^{1} \mathrm{H}$ RMN NOE DIFF do composto 5a $\left(\mathrm{CDCl}_{3}\right)$.

\begin{tabular}{c|c|c}
\hline$\delta(\mathbf{p p m})$ Irradiado & Atribuição & Efeito NOE Observado \\
\hline 1,13 & $\mathrm{CH}_{3}(7)$ & $\mathrm{Hb}, \mathrm{Hd}$ \\
1,06 & $\mathrm{CH}_{3}(6)$ & $\mathrm{Ha}, \mathrm{Hc}, \mathrm{He}$ \\
1,04 & $\mathrm{CH}_{3}(8)$ & $\mathrm{Ha}, \mathrm{He}$ \\
\hline
\end{tabular}

Tabela 12- Dados espectrais de ${ }^{1} \mathrm{H}$ e ${ }^{13} \mathrm{C}$ RMN para o $( \pm)$ - cis - 2 - Dimetoxi-2,4,4trimetil-ciclopentanol $\left(\mathrm{CDCl}_{3}\right)$

\begin{tabular}{|c|c|c|}
\hline $\mathrm{C}-, \mathrm{H}_{-}$ & ${ }^{1} \mathrm{H}(300 \mathrm{MHz}) \delta(\mathrm{ppm})$ & ${ }^{13} \mathrm{C}(75 \mathrm{MHz})$ \\
\hline 1 & $\mathrm{He}-3,94(\mathrm{t}, 1 \mathrm{H}, \mathrm{Jec}=\mathrm{Jed}=5 \mathrm{~Hz})$ e $2,82(\mathrm{sl}, 1 \mathrm{H}, \mathrm{OH})$ & 81,64 \\
\hline 2 & $\begin{array}{c}\mathrm{Hc}-1,84(\mathrm{ddd}, 1 \mathrm{H}, \mathrm{Jcd}=13,2 ; \mathrm{Jce}=5 \text { e Jca }=1,3 \mathrm{~Hz}) \text { e Hd-1,57 } \\
(\mathrm{dd}, 1 \mathrm{H}, \mathrm{Jdc}=13,2 \text { e Jde=5 Hz) }\end{array}$ & 49,03 \\
\hline 3 & & 36,20 \\
\hline 4 & $\mathrm{Hb}-1,89(\mathrm{~d}, 1 \mathrm{H}, \mathrm{Jba}=13,3 \mathrm{~Hz})$ e Ha- $1,26(\mathrm{dd}, 1 \mathrm{H}, \mathrm{Jab}=13,3 \mathrm{e}$ & 48,40 \\
\hline 5 & $\mathrm{Jac}=1,3 \mathrm{~Hz})$ & 51,63 \\
\hline 6 & $1,06(\mathrm{~s}, 3 \mathrm{H})$ & 32,27 \\
\hline 7 & $1,13(\mathrm{~s}, 3 \mathrm{H})$ & 32,75 \\
\hline 8 & $1,04(\mathrm{~s}, 3 \mathrm{H})$ & 21,76 \\
\hline 9 & $4,27(\mathrm{~s}, 1 \mathrm{H})$ & 111,43 \\
\hline 10 & $3,51(\mathrm{~s}, 3 \mathrm{H})$ & 58,17 \\
\hline 11 & $3,53(\mathrm{~s}, 3 \mathrm{H})$ & 57,68 \\
\hline
\end{tabular}




\section{Composto (5b)}<smiles>[CH][C@@H]1C(C)(C)[C@@H](C)[C@@H](O)[C@@]1(C)C(=O)OC</smiles>

\section{Espectro de ${ }^{1} \mathrm{H}-\mathrm{RMN}$ do compost o (5b)}

$5 \mathbf{b}$

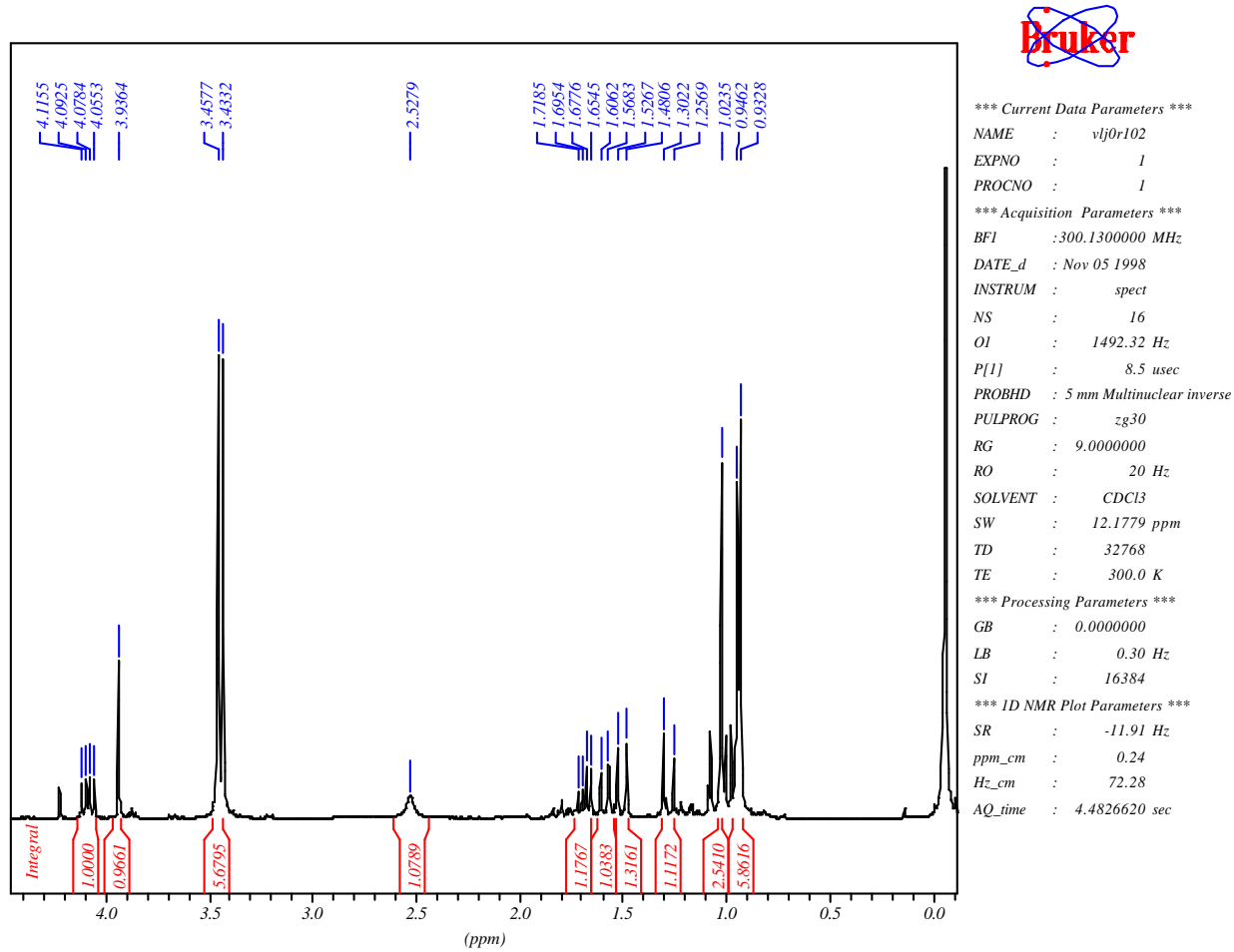

Tabela 13 - Dados espectrais de ${ }^{1} \mathrm{H}$ RMN do composto $\mathbf{5 b}\left(\mathrm{CDCl}_{3}\right)$.

\begin{tabular}{c|c|c|c}
\hline$\delta(\mathbf{p p m})$ & Atribuição & sinal & J (Hz) \\
\hline 4,08 & $\mathrm{He}$ & $\mathrm{dd}$ & Jec=11,3 e Jed=6,9 Hz \\
3,93 & 9 & $\mathrm{~s}$ & \\
3,45 & 11 & $\mathrm{~s}$ & \\
3,43 & 10 & $\mathrm{~s}$ & \\
2,52 & $\mathrm{OH}$ & $\mathrm{sl}$ & \\
1,68 & $\mathrm{Hd}$ & $\mathrm{dd}$ & $\mathrm{Jdc}=12,2 \mathrm{e} \mathrm{Jde}=6,9 \mathrm{~Hz}$ \\
1,57 & $\mathrm{Hc}$ & $\mathrm{dd}$ & $\mathrm{Jcd}=12,2 \mathrm{e} \mathrm{Jce}=11,3 \mathrm{~Hz}$ \\
1,50 & $\mathrm{Hb}$ & $\mathrm{d}$ & $\mathrm{Jba}=13,6 \mathrm{~Hz}$ \\
1,28 & $\mathrm{Ha}$ & $\mathrm{d}$ & $\mathrm{Jab}=13,6 \mathrm{~Hz}$ \\
1,02 & $\mathrm{CH}_{3}(6)$ & $\mathrm{s}$ & \\
0,94 & $\mathrm{CH}_{3}(7)$ & $\mathrm{s}$ & \\
0,93 & $\mathrm{CH}_{3}(8)$ & $\mathrm{s}$ & \\
\hline
\end{tabular}




\section{Espectro de ${ }^{13} \mathrm{C}$-RMN (BB) do composto (5b)}

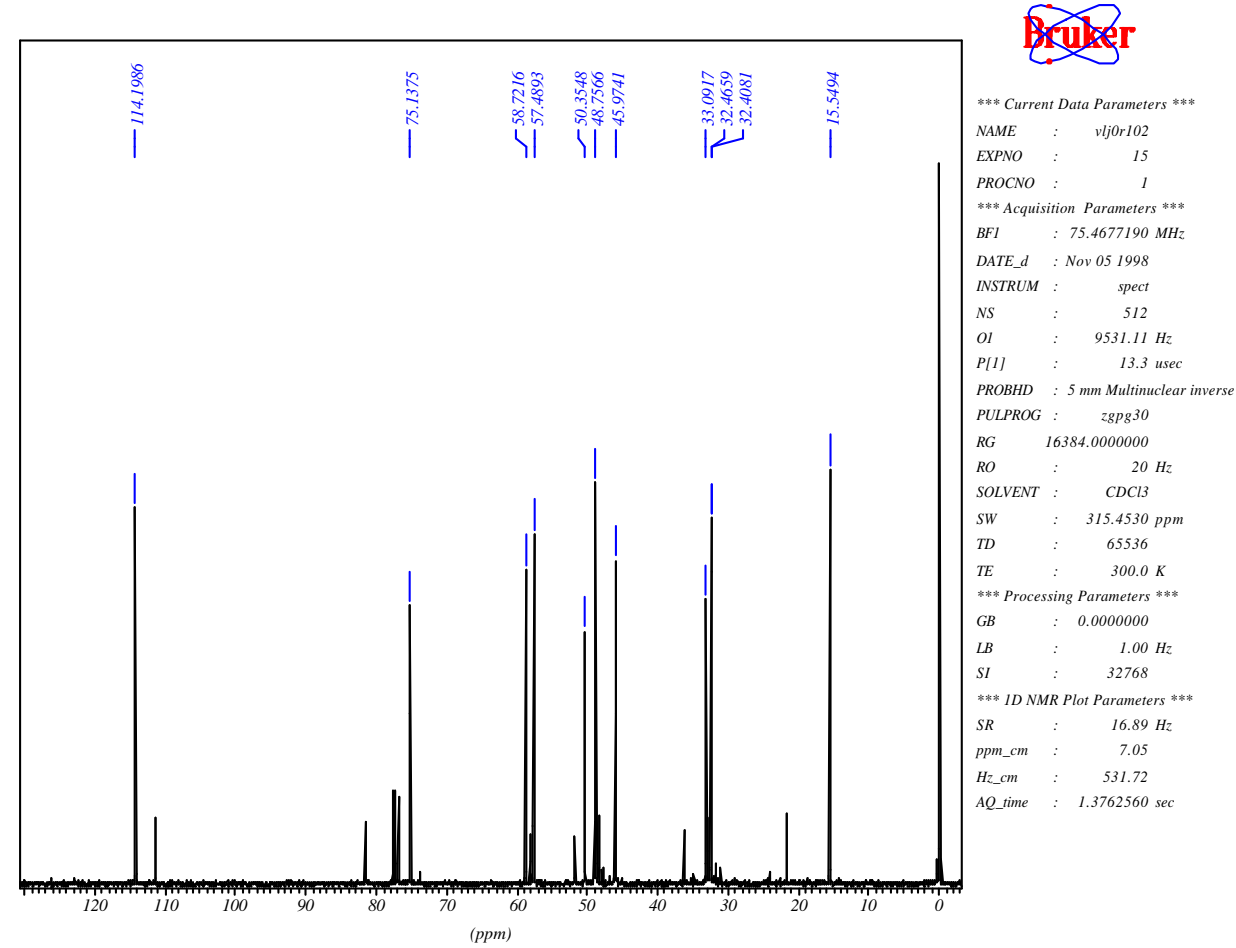

\section{Espectro de ${ }^{13}$ C-RMN (DEPT-135) do composto (5b)}

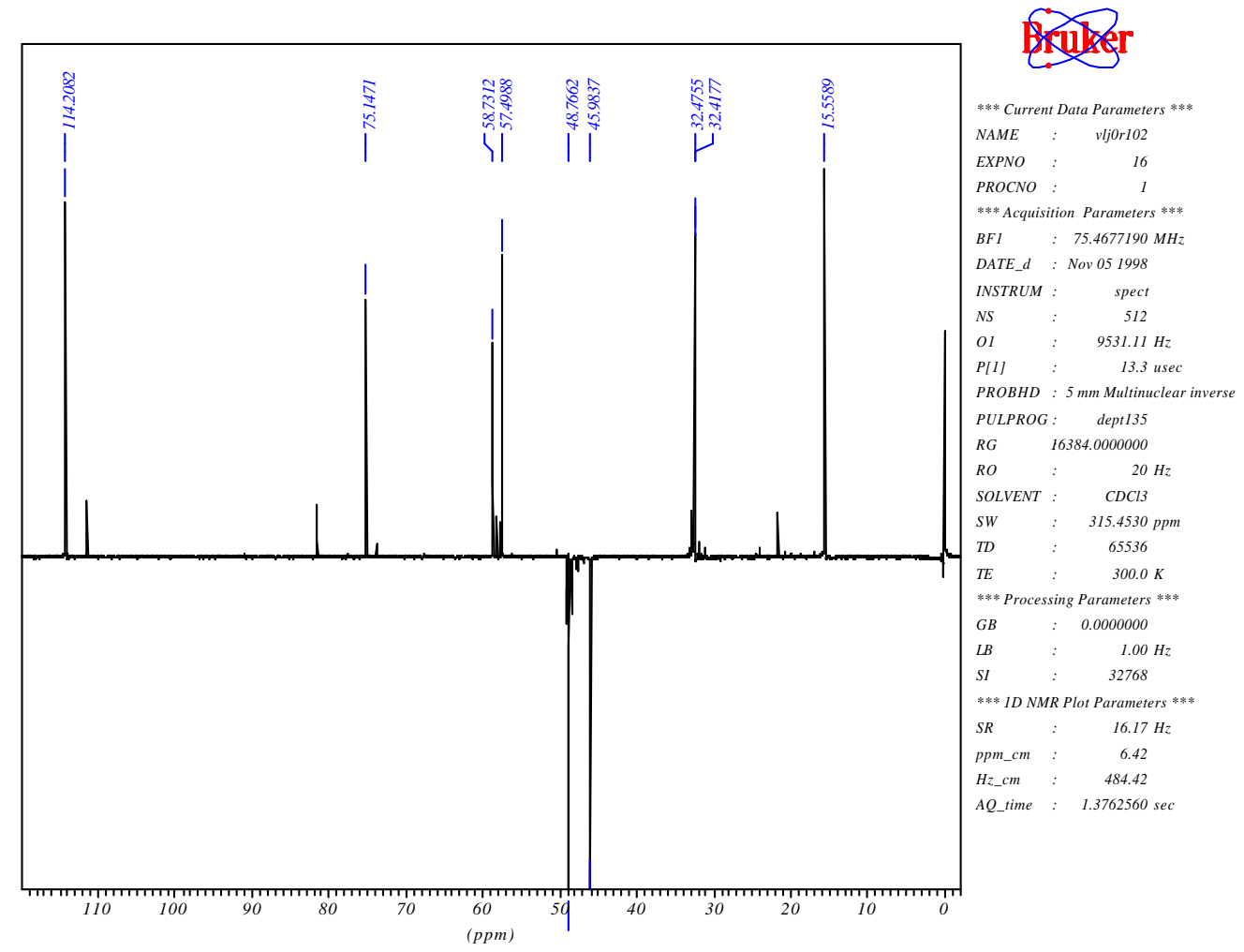




\section{Espectro HMQC (2D H/C) do composto (5b)}

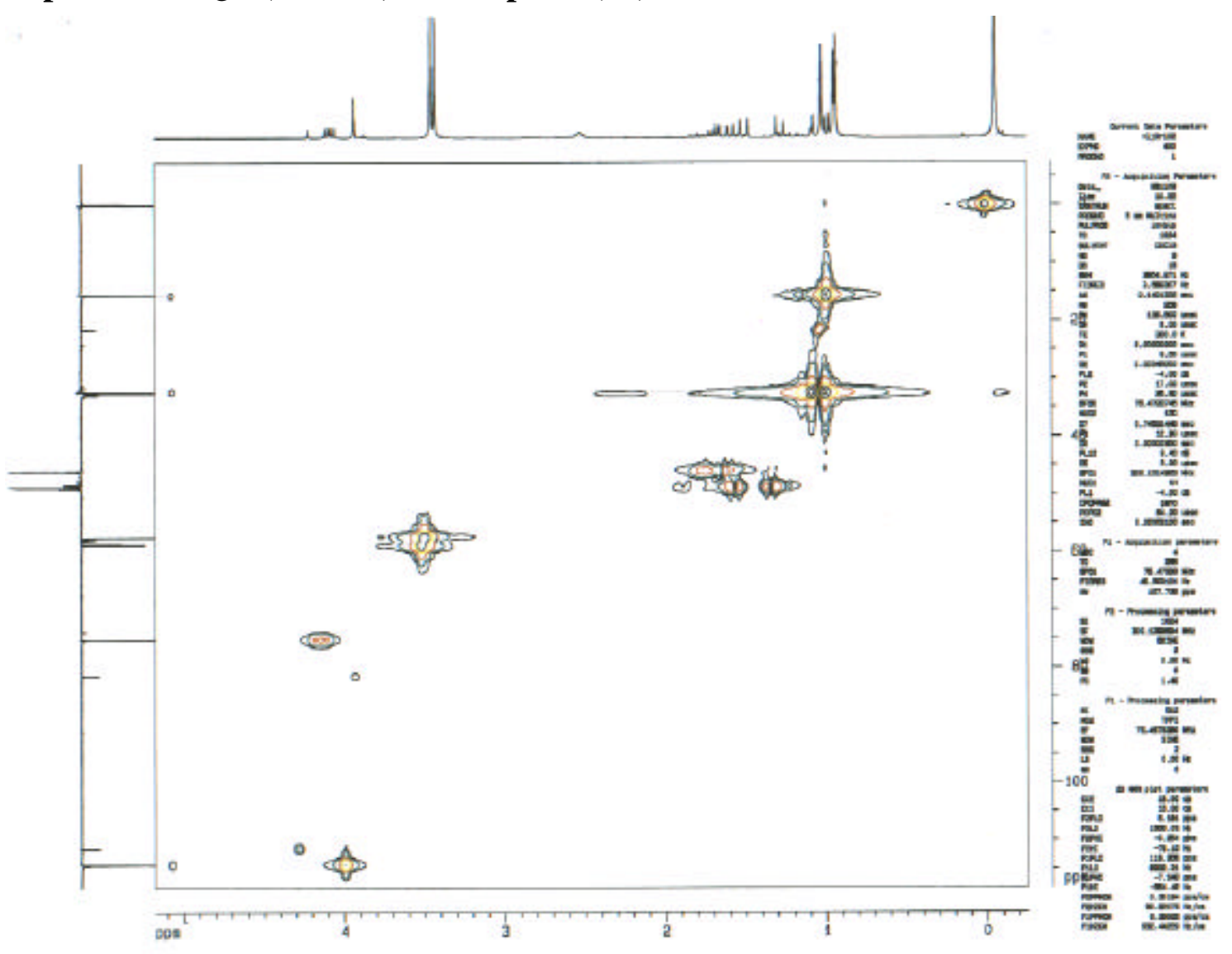

\section{Espectros NOE DIFF do composto (5b)}

irradiação na metila 6
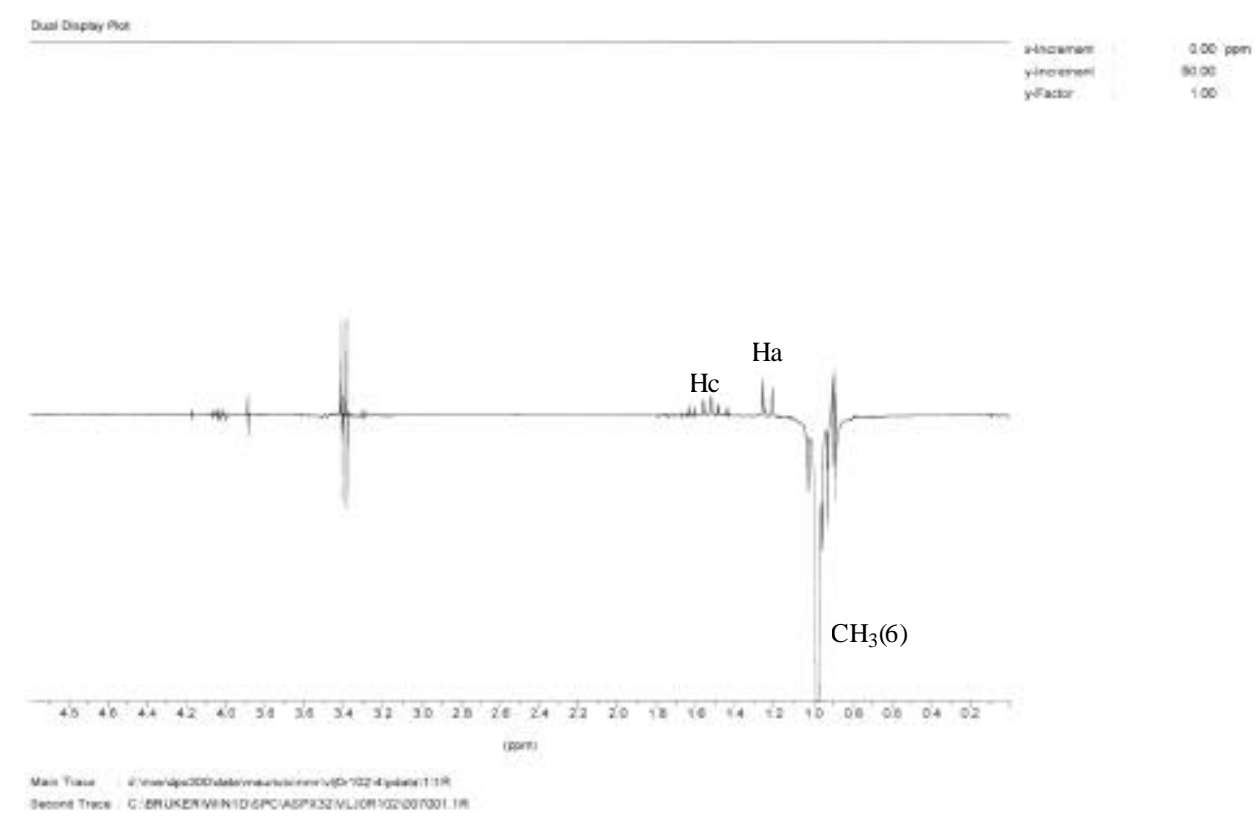
irradiação na metila 7

and Dever ate

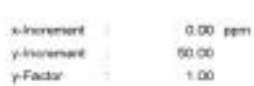

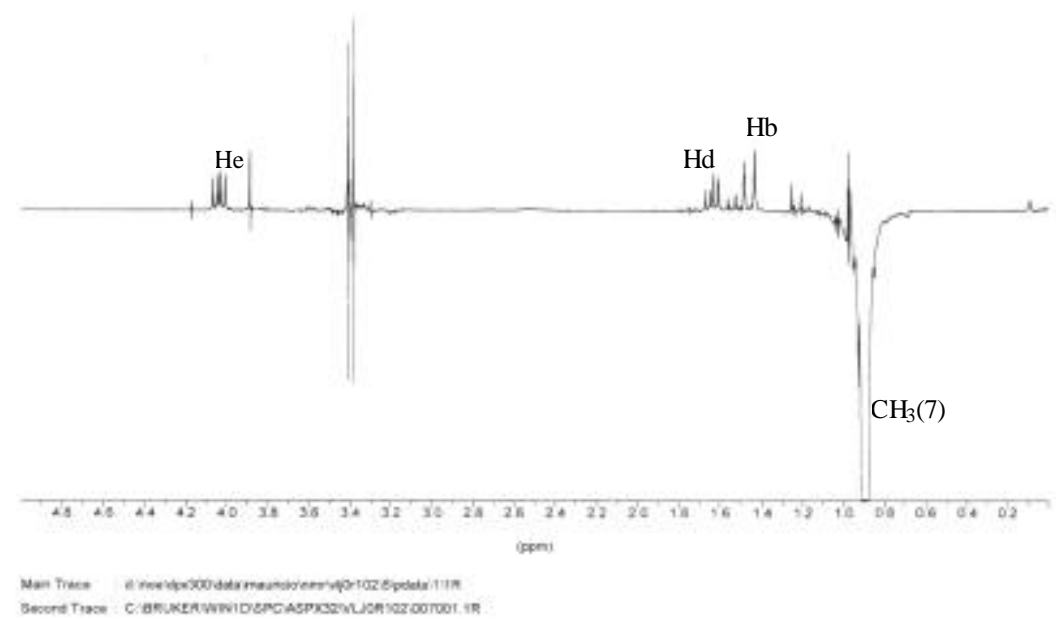

irradiação na metila 8

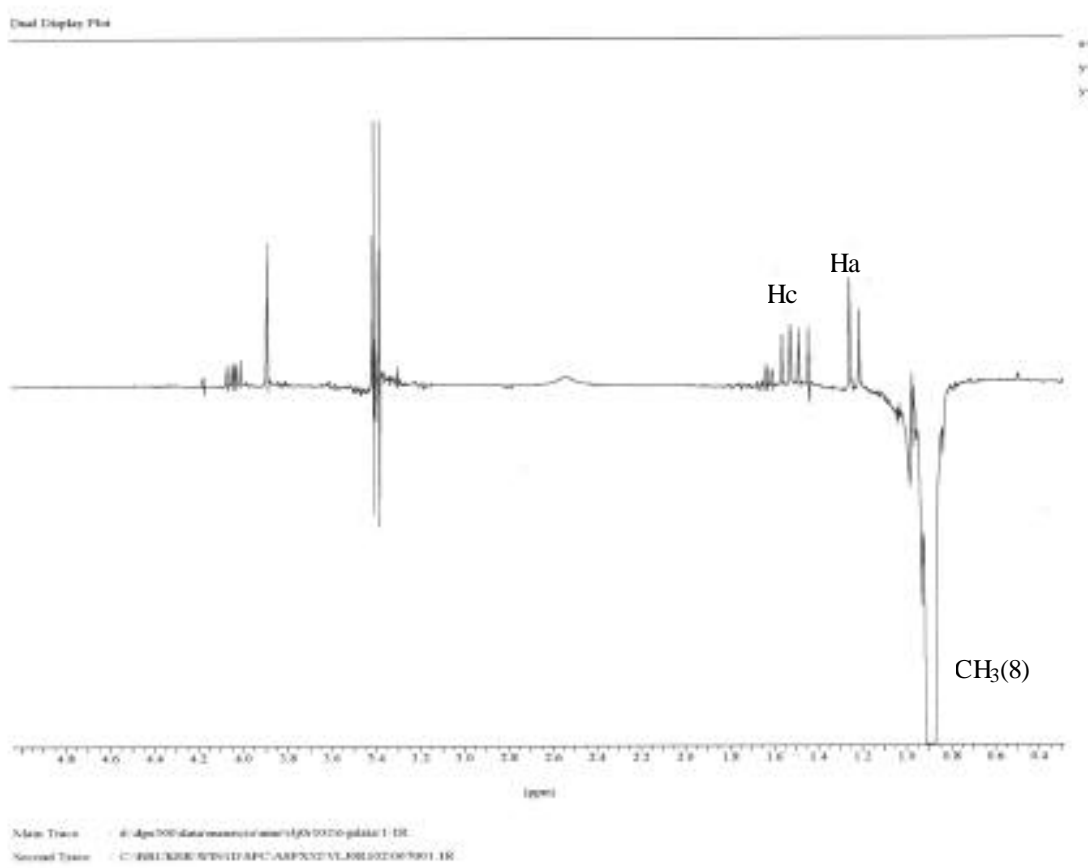


Tabela 14 - Dados espectrais de NOE DIFF do composto $\mathbf{5 b}\left(\mathrm{CDCl}_{3}\right)$.

\begin{tabular}{c|c|c}
\hline$\delta(\mathbf{p p m})$ Irradiado & Atribuição & Efeito NOE Observado \\
\hline 1,02 & $\mathrm{CH}_{3}(6)$ & $\mathrm{Ha}, \mathrm{Hc}$ \\
0,94 & $\mathrm{CH}_{3}(7)$ & $\mathrm{Hb}, \mathrm{Hd}, \mathrm{He}$ \\
0,93 & $\mathrm{CH}_{3}(8)$ & $\mathrm{Ha}, \mathrm{Hc}$ \\
\hline
\end{tabular}

Tabela 15 - Dados espectrais de ${ }^{1} \mathrm{H}$ e ${ }^{13} \mathrm{C} \mathrm{RMN} \mathrm{para} \mathrm{o}( \pm)$ - trans - 2 - Dimetoxi2,4,4-trimetil-ciclopentanol $\left(\mathrm{CDCl}_{3}\right)$

\begin{tabular}{|c|c|c|}
\hline $\mathrm{C}-, \mathrm{H}-$ & ${ }^{1} \mathrm{H}(300 \mathrm{MHz}) \delta(\mathrm{ppm})$ & ${ }^{3} \mathrm{C}(75 \mathrm{MHz})$ \\
\hline 1 & $\mathrm{He}-4,08(\mathrm{dd}, 1 \mathrm{H}, \mathrm{Jec}=11,3$ e Jed=6,9 Hz) e 2,52 (sl, 1H, OH) & 75,13 \\
\hline 2 & $\mathrm{Hd}-1,68(\mathrm{dd}, 1 \mathrm{H}, \mathrm{Jdc}=12,2$ e Jde=6,9 Hz) e Hc-1,57 (dd, 1H, & 45,97 \\
& $\mathrm{Jcd}=12,2$ e Jce $=11,3 \mathrm{~Hz})$ & \\
\hline 3 & & 36,09 \\
\hline 4 & $\mathrm{Hb}-1,50(\mathrm{~d}, 1 \mathrm{H}, \mathrm{Jba}=13,6 \mathrm{~Hz})$ e Ha-1,28 (d, 1H, Jab=13,6Hz) & 48,75 \\
\hline 5 & & 50,35 \\
\hline 6 & $1,02(\mathrm{~s}, 3 \mathrm{H})$ & 32,40 \\
\hline 7 & $0,94(\mathrm{~s}, 3 \mathrm{H})$ & 32,46 \\
\hline 8 & $0,93(\mathrm{~s}, 3 \mathrm{H})$ & 15,55 \\
\hline 9 & $3,93(\mathrm{~s}, 1 \mathrm{H})$ & 114,19 \\
\hline 10 & $3,43(\mathrm{~s}, 3 \mathrm{H})$ & 58,72 \\
\hline 11 & $3,45(\mathrm{~s}, 3 \mathrm{H})$ & 57,49 \\
\hline
\end{tabular}




\section{Composto (6a)}

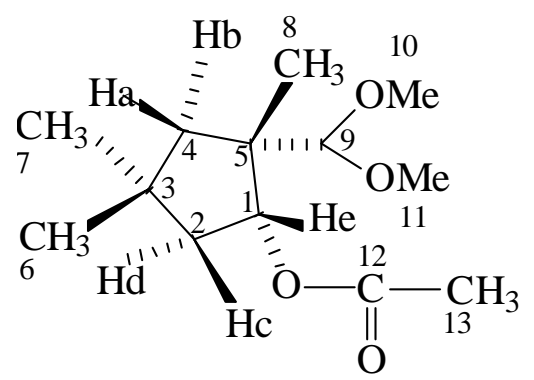

Espectro de ${ }^{1} \mathrm{H}-\mathrm{RMN}$ do composto (6a)

6a

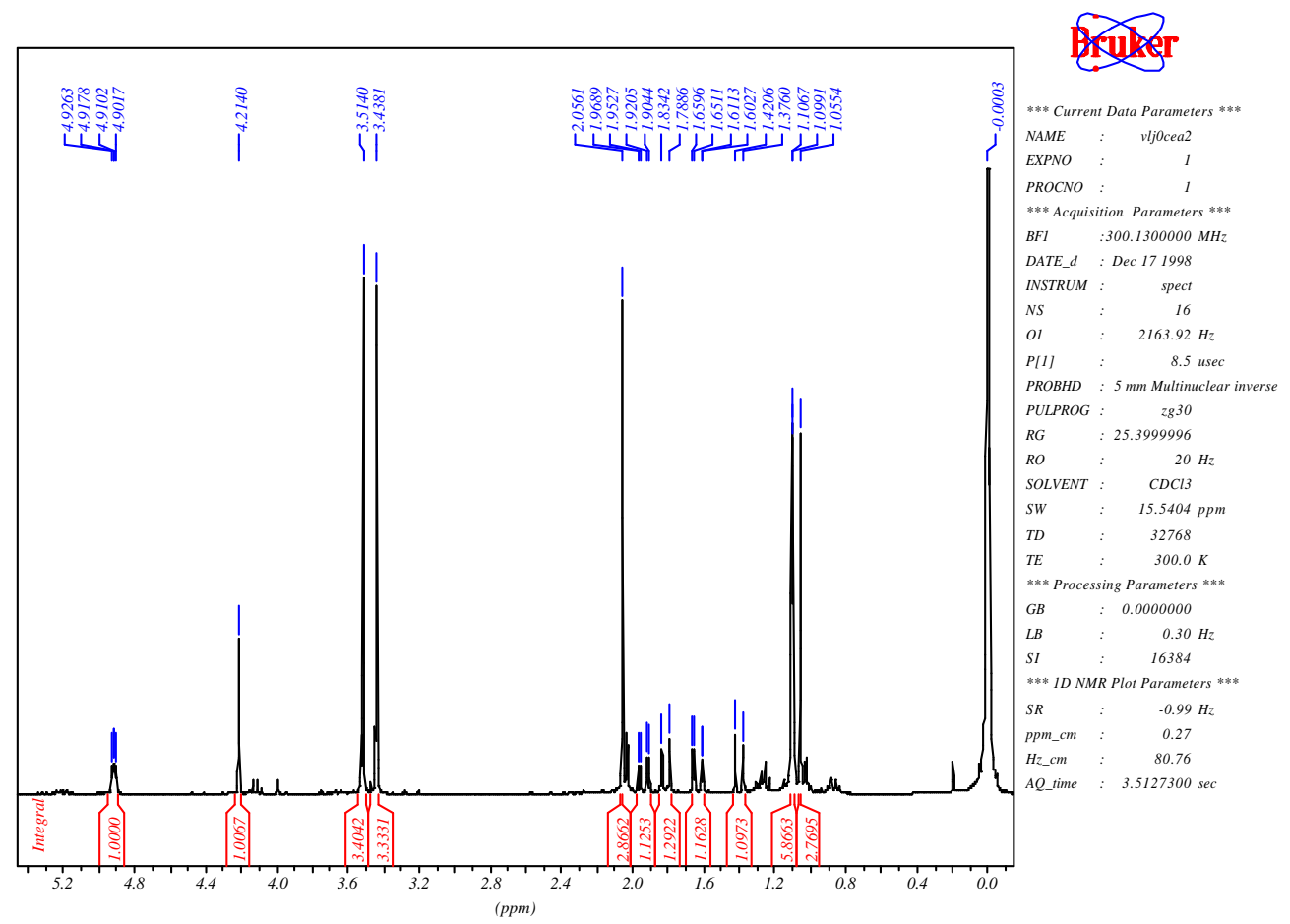

Tabela 16 - Dados espectrais de ${ }^{1} \mathrm{H}$ RMN do composto $\mathbf{6 a}\left(\mathrm{CDCl}_{3}\right)$.

\begin{tabular}{c|c|c|c}
\hline$\delta(\mathbf{p p m})$ & Atribuição & sinal & $\mathbf{J}(\mathbf{H z})$ \\
\hline 4,91 & $\mathrm{He}$ & $\mathrm{dd}$ & $\mathrm{Jec}=4,8 \mathrm{e} \mathrm{Jed}=2,5 \mathrm{~Hz}$ \\
4,21 & 9 & $\mathrm{~s}$ & \\
3,43 & 11 & $\mathrm{~s}$ & \\
3,51 & 10 & $\mathrm{~s}$ & \\
2,05 & 13 & $\mathrm{~s}$ & $\mathrm{ddd}$ \\
1,93 & $\mathrm{Hcd}=14,5 ; \mathrm{Jce}=4,8 \mathrm{e} \mathrm{Jca}=1,3 \mathrm{~Hz}$ \\
1,81 & $\mathrm{Hb}$ & $\mathrm{d}$ & $\mathrm{Jba}=13,6 \mathrm{~Hz}$ \\
1,63 & $\mathrm{Hd}$ & $\mathrm{dd}$ & $\mathrm{Jdc}=14,5 \mathrm{e} \mathrm{Jde}=2,5 \mathrm{~Hz}$ \\
1,39 & $\mathrm{Ha}$ & $\mathrm{dd}$ & $\mathrm{Jab}=13,6 \mathrm{e} \mathrm{Jac}=1,3 \mathrm{~Hz}$ \\
1,10 & $\mathrm{CH}_{3}(6)$ & $\mathrm{s}$ & \\
1,09 & $\mathrm{CH}_{3}(7)$ & $\mathrm{s}$ & \\
1,05 & $\mathrm{CH}_{3}(8)$ & $\mathrm{s}$ & \\
\hline
\end{tabular}


Espectro de ${ }^{13} \mathrm{C}-\mathrm{RMN}$ (BB) do composto (6a)

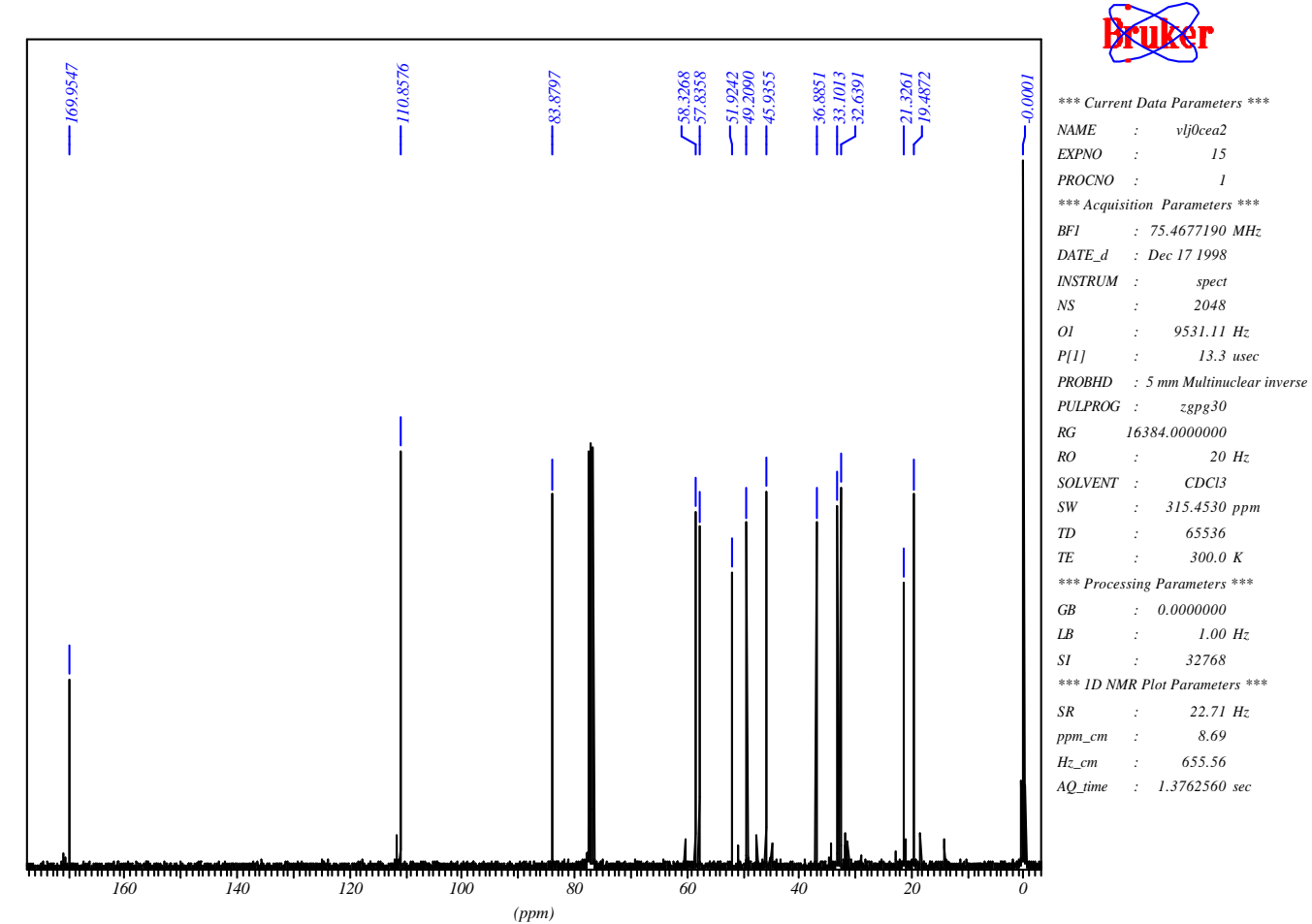

Espectro de ${ }^{13}$ C-RMN (DEPT-135) do composto (6a)

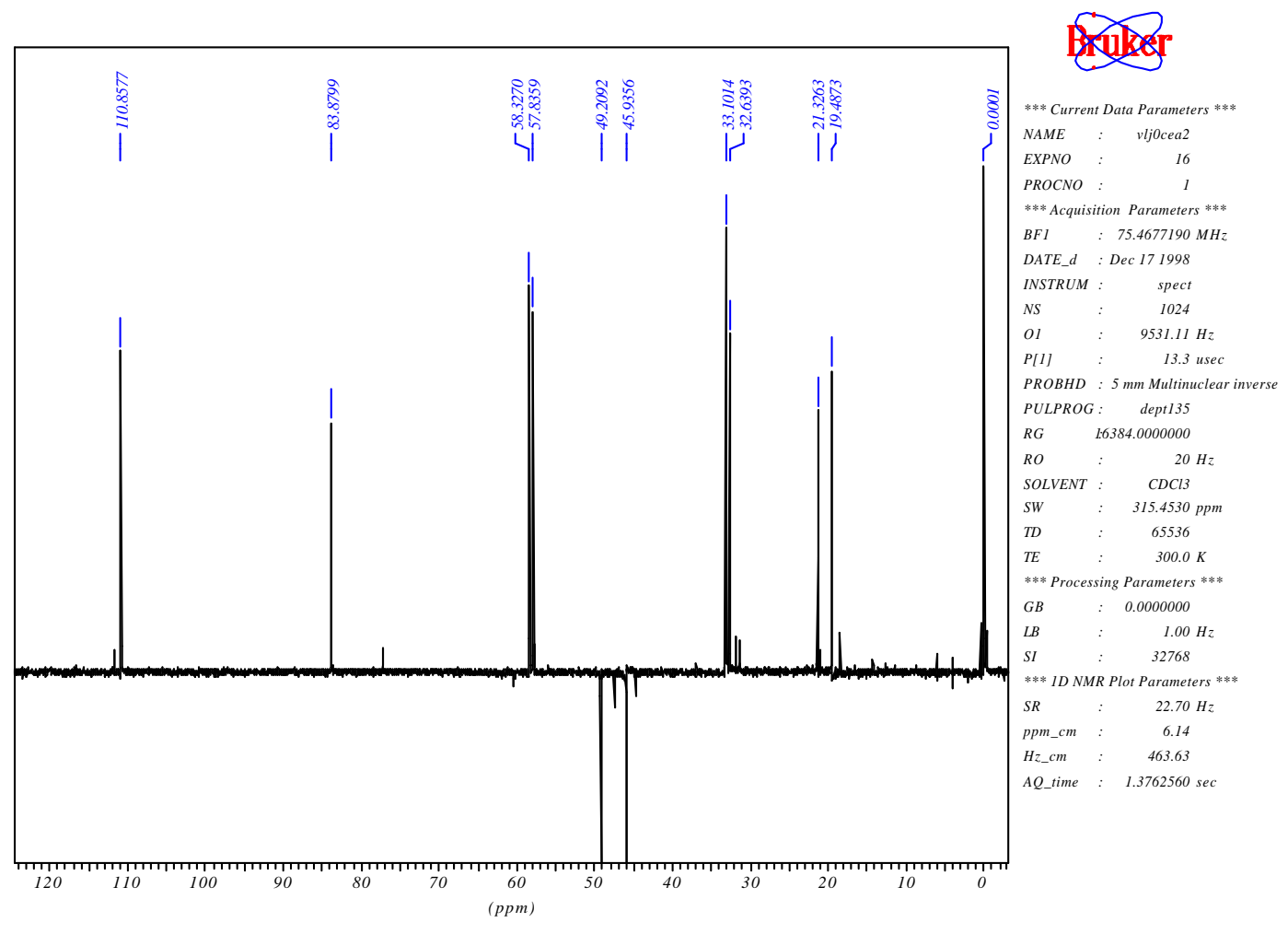




\section{Espectro HMQC (2D H/C) do composto (6a)}

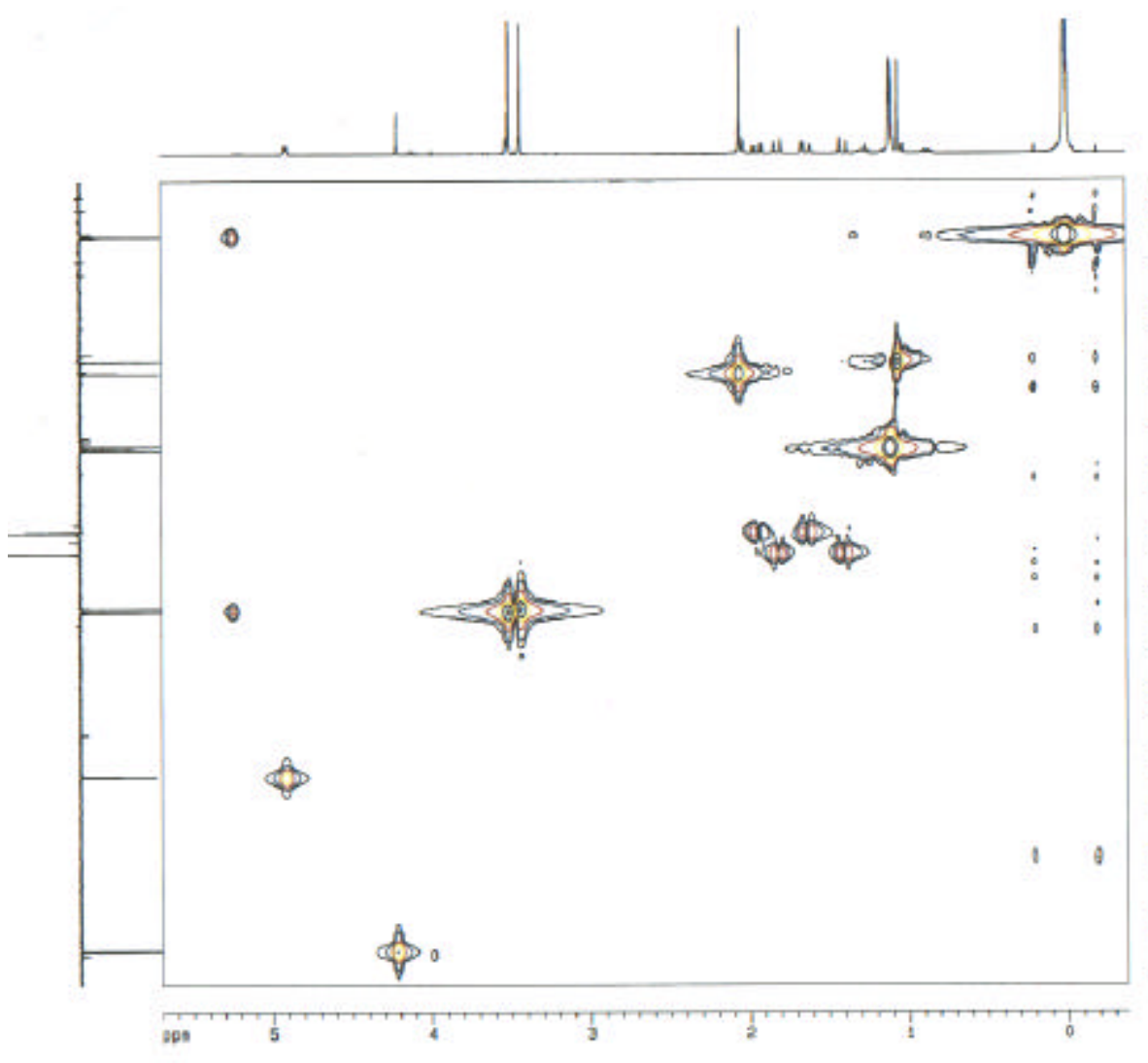

Espectro Jres do composto (6a)
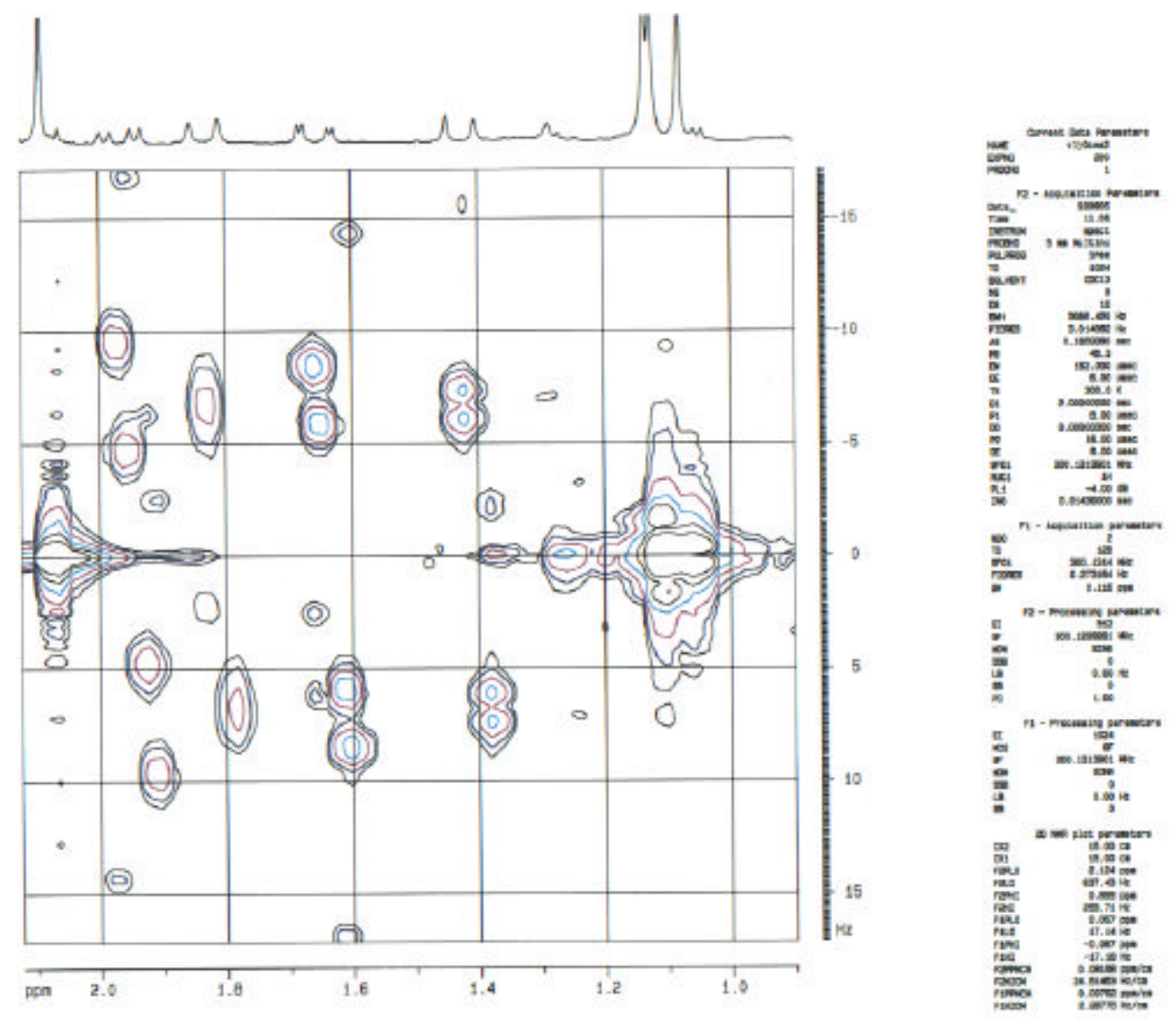


\section{Espectros NOE DIFF do composto (6a)}

$>$ irradiação na metila 6

Dual Display Plot

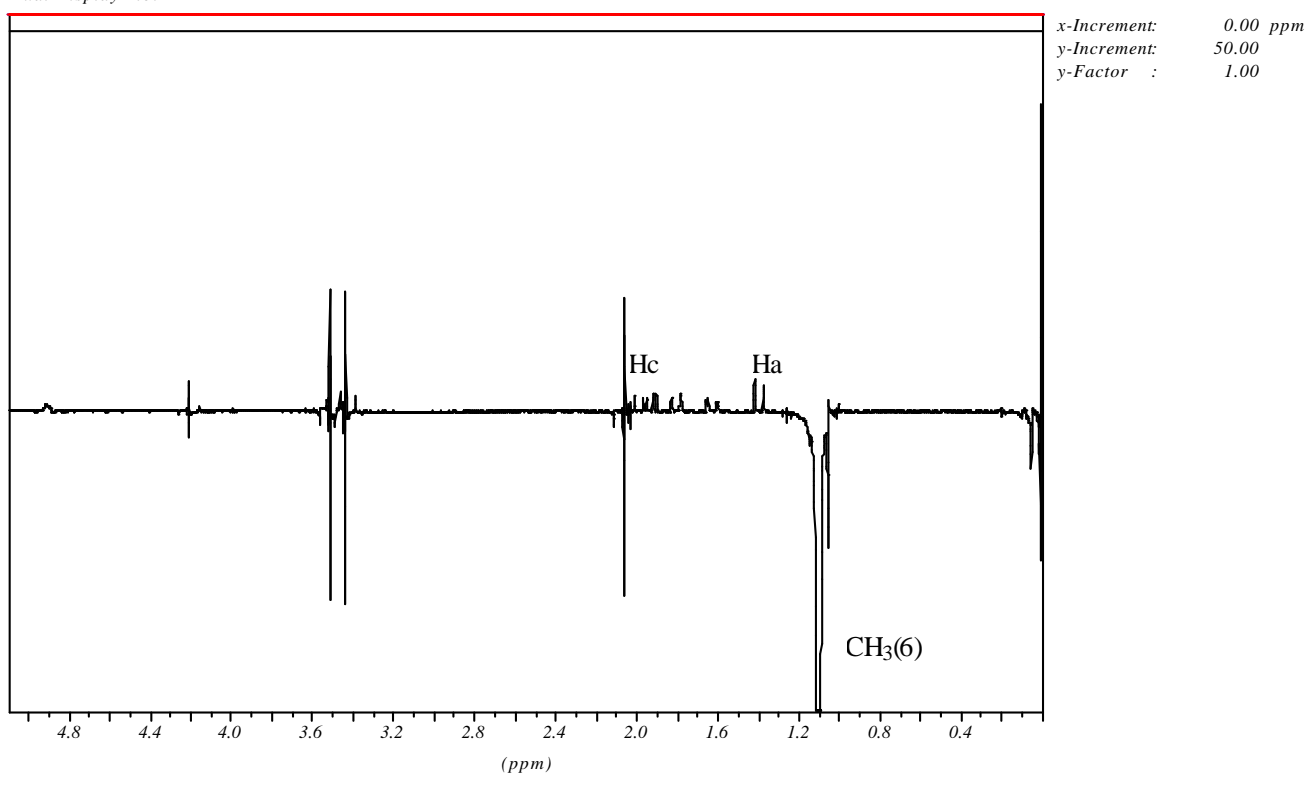

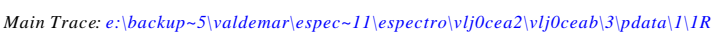
Second Trace: $\backslash$ WINID $\backslash S P C \backslash A S P X 32 \backslash V L J O C E A B \backslash 006001.1 R$

irradiação na metila 7

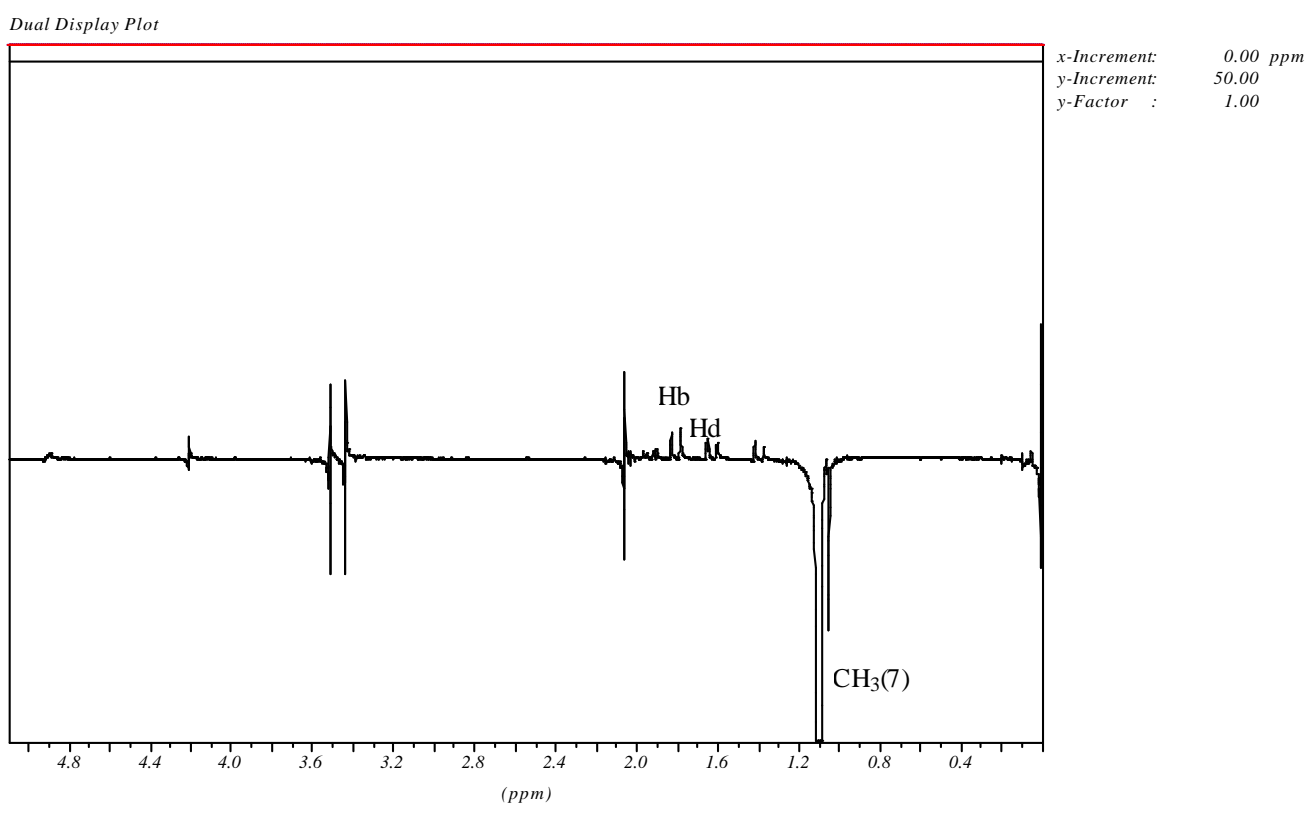

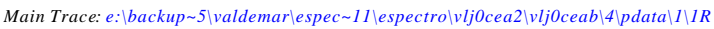

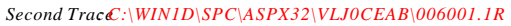


irradiação na metila 8

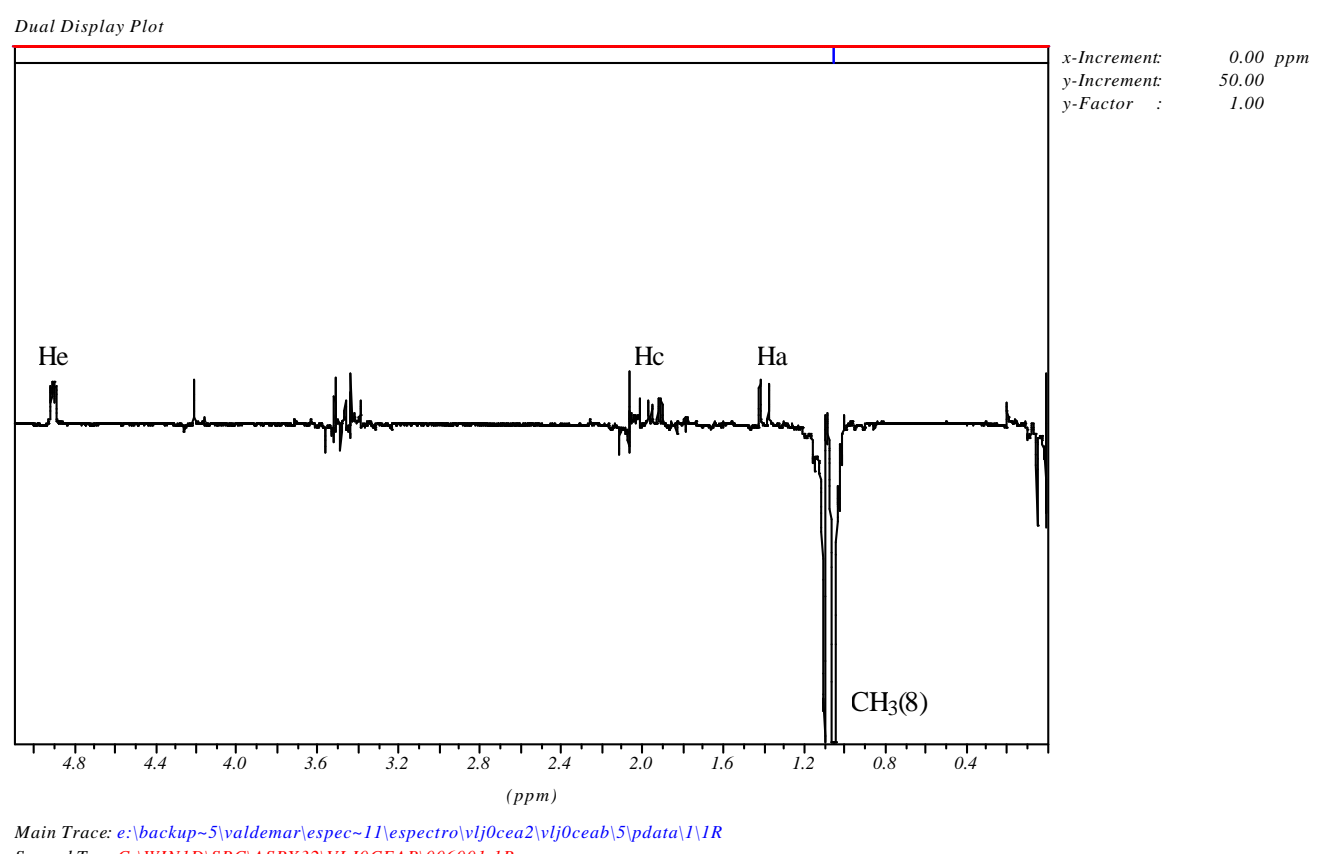

Second Trace $: \backslash W I N 1 D \backslash S P C \backslash A S P X 32 \backslash V L J O C E A B \backslash 006001.1 R$

Tabela 17 - Dados espectrais de NOE DIFF do composto $6 \mathbf{a}\left(\mathrm{CDCl}_{3}\right)$.

\begin{tabular}{c|c|c}
\hline$\delta(\mathbf{p p m})$ Irradiado & Atribuição & Efeito NOE Observado \\
\hline 1,10 & $\mathrm{CH}_{3}(6)$ & $\mathrm{Ha}, \mathrm{Hc}$ \\
1,09 & $\mathrm{CH}_{3}(7)$ & $\mathrm{Hb}, \mathrm{Hd}$ \\
1,05 & $\mathrm{CH}_{3}(8)$ & $\mathrm{Ha}, \mathrm{Hc}, \mathrm{He}$ \\
\hline
\end{tabular}

Tabela 18- Dados espectrais de ${ }^{1} \mathrm{H}$ e ${ }^{13} \mathrm{C} \mathrm{RMN}$ para o $( \pm)$ - cis - 1 - Dimetoximetil-2acetoxi - 1,4,4 - trimetil-ciclopentano $\left(\mathrm{CDCl}_{3}\right)$

\begin{tabular}{|c|c|c|}
\hline $\mathrm{C}-, \mathrm{H}-$ & ${ }^{1} \mathrm{H}(300 \mathrm{MHz}) \delta(\mathrm{ppm})$ & ${ }^{{ }^{13} \mathrm{C}}$ \\
\hline 1 & $\mathrm{He}-4,91(\mathrm{dd}, 1 \mathrm{H}, \mathrm{Jec}=4,8$ e Jed=2,5 Hz) & 83,8 \\
\hline 2 & $\begin{array}{c}\text { Hc-1,93 (ddd, } 1 \mathrm{H}, \mathrm{Jcd}=14,5 ; \mathrm{Jce}=4,8 \text { e Jca }=1,3 \mathrm{~Hz}) \text { e Hd-1,63 } \\
(\mathrm{dd}, 1 \mathrm{H}, \mathrm{Jdc}=14,5 \text { e Jde }=2,5 \mathrm{~Hz})\end{array}$ & 45,9 \\
\hline 3 & & 36,8 \\
\hline 4 & $\begin{array}{l}\mathrm{Hb}-1,81(\mathrm{~d}, 1 \mathrm{H}, \mathrm{Jba}=13,6 \mathrm{~Hz}) \text { e } \mathrm{Ha}-1,39(\mathrm{dd}, 1 \mathrm{H}, \mathrm{Jab}=13,6 \mathrm{e} \\
\qquad \mathrm{Jac}=1,3 \mathrm{~Hz})\end{array}$ & 49,2 \\
\hline 5 & & 51,9 \\
\hline 6 & $1,10(\mathrm{~s}, 3 \mathrm{H})$ & 33,1 \\
\hline 7 & $1,09(\mathrm{~s}, 3 \mathrm{H})$ & 32,6 \\
\hline 8 & $1,05(\mathrm{~s}, 3 \mathrm{H})$ & 19,4 \\
\hline 9 & $4,21(\mathrm{~s}, 1 \mathrm{H})$ & 110,8 \\
\hline 10 & $3,51(\mathrm{~s}, 3 \mathrm{H})$ & 58,3 \\
\hline 11 & $3,43(\mathrm{~s}, 3 \mathrm{H})$ & 57,8 \\
\hline
\end{tabular}




\begin{tabular}{|c|c|c|}
\hline 12 & & 169,9 \\
\hline 13 & $2,05(\mathrm{~s}, 3 \mathrm{H})$ & 21,3 \\
\hline
\end{tabular}

\section{Composto (6b)}

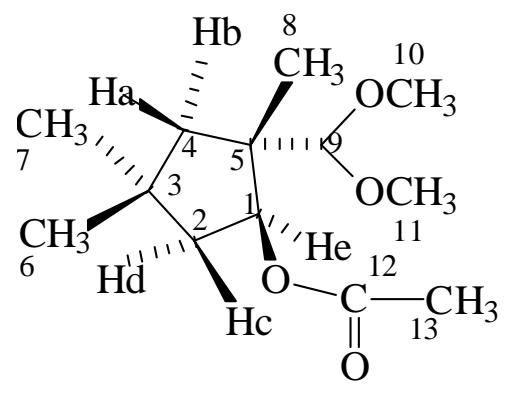

Espectro de ${ }^{1} \mathrm{H}-\mathrm{RMN}$ do composto (6b)

6b

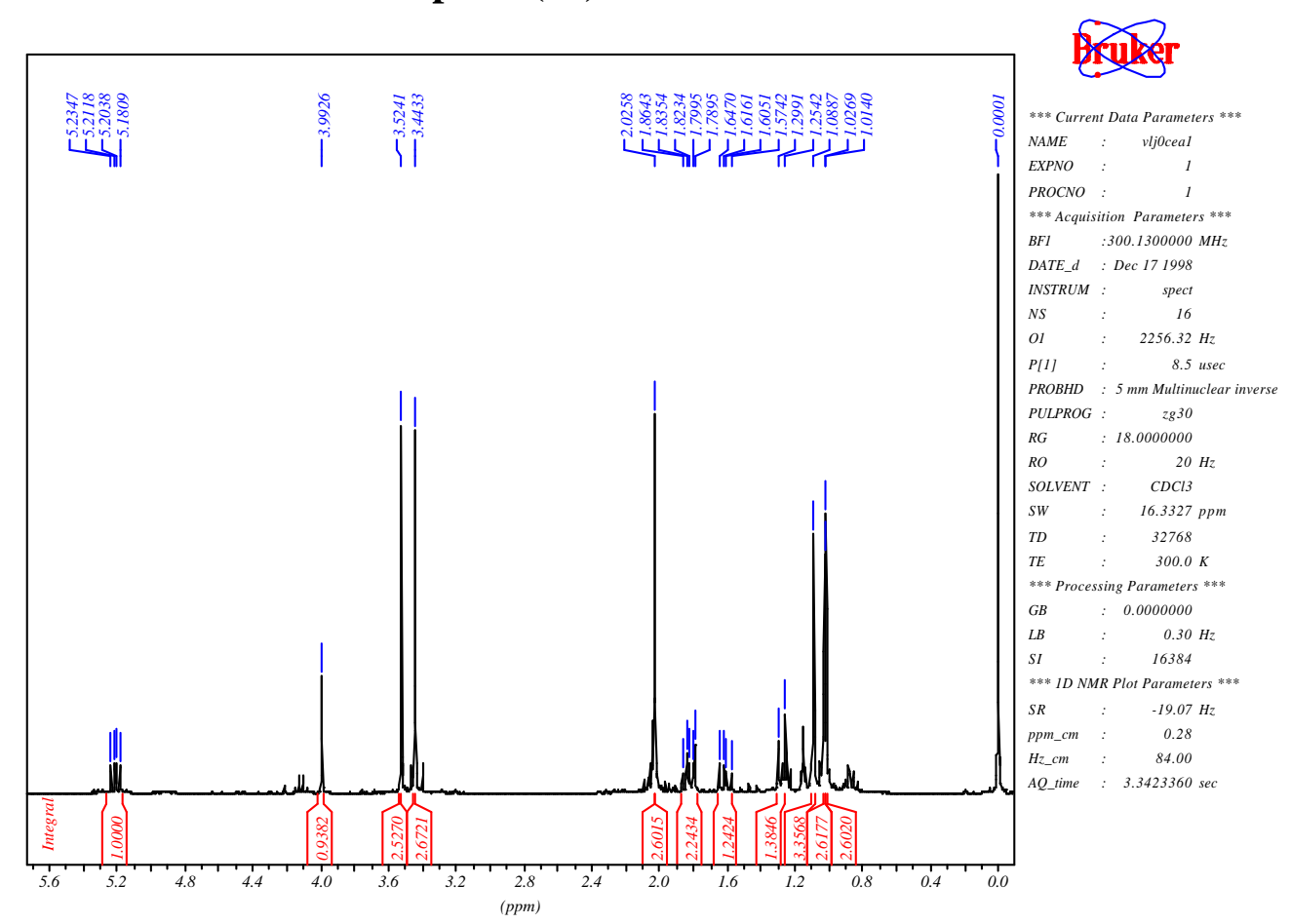

Tabela 19 - Dados espectrais de ${ }^{1} \mathrm{H}$ RMN do composto $\mathbf{6 b}\left(\mathrm{CDCl}_{3}\right)$.

\begin{tabular}{c|c|c|c}
\hline$\delta(\mathbf{p p m})$ & Atribuição & sinal & J (Hz) \\
\hline 5,2 & He & $\mathrm{dd}$ & $\mathrm{Jec}=9,3 \mathrm{e} \mathrm{Jed}=6,9 \mathrm{~Hz}$ \\
3,99 & 9 & $\mathrm{~s}$ & \\
3,52 & 10 & $\mathrm{~s}$ & \\
3,44 & 11 & $\mathrm{~s}$ & \\
2,02 & 13 & $\mathrm{~s}$ & \\
1,83 & $\mathrm{Hd}$ & $\mathrm{ddd}$ & $\mathrm{Jdc}=12,4 ; \mathrm{Jde}=6,9 \mathrm{e} \mathrm{Jdb}=1,3 \mathrm{~Hz}$ \\
1,81 & $\mathrm{Hb}$ & $\mathrm{dd}$ & $\mathrm{Jba}=13,7 \mathrm{e} \mathrm{Jbd}=1,3 \mathrm{~Hz}$ \\
1,61 & $\mathrm{Hc}$ & $\mathrm{ddd}$ & $\mathrm{Jcd}=12,4 ; \mathrm{Jce}=9,3 \mathrm{e} \mathrm{Jca}=0,5 \mathrm{~Hz}$ \\
\hline
\end{tabular}

Aplicações de Técnicas de RMN À Determinação Estrutural de Intermediários Sintéticos 


\begin{tabular}{c|c|c|l}
\hline 1,27 & $\mathrm{Ha}$ & $\mathrm{dd}$ & $\mathrm{Jab}=13,7$ e Jac $=0,5 \mathrm{~Hz}$ \\
1,08 & $\mathrm{CH}_{3}(6)$ & $\mathrm{s}$ & \\
1,02 & $\mathrm{CH}_{3}(7)$ & $\mathrm{s}$ & \\
1,01 & $\mathrm{CH}_{3}(8)$ & $\mathrm{s}$ & \\
\hline
\end{tabular}

Espectro de ${ }^{13} \mathrm{C}-\mathrm{RMN}$ (BB) do composto (6b)

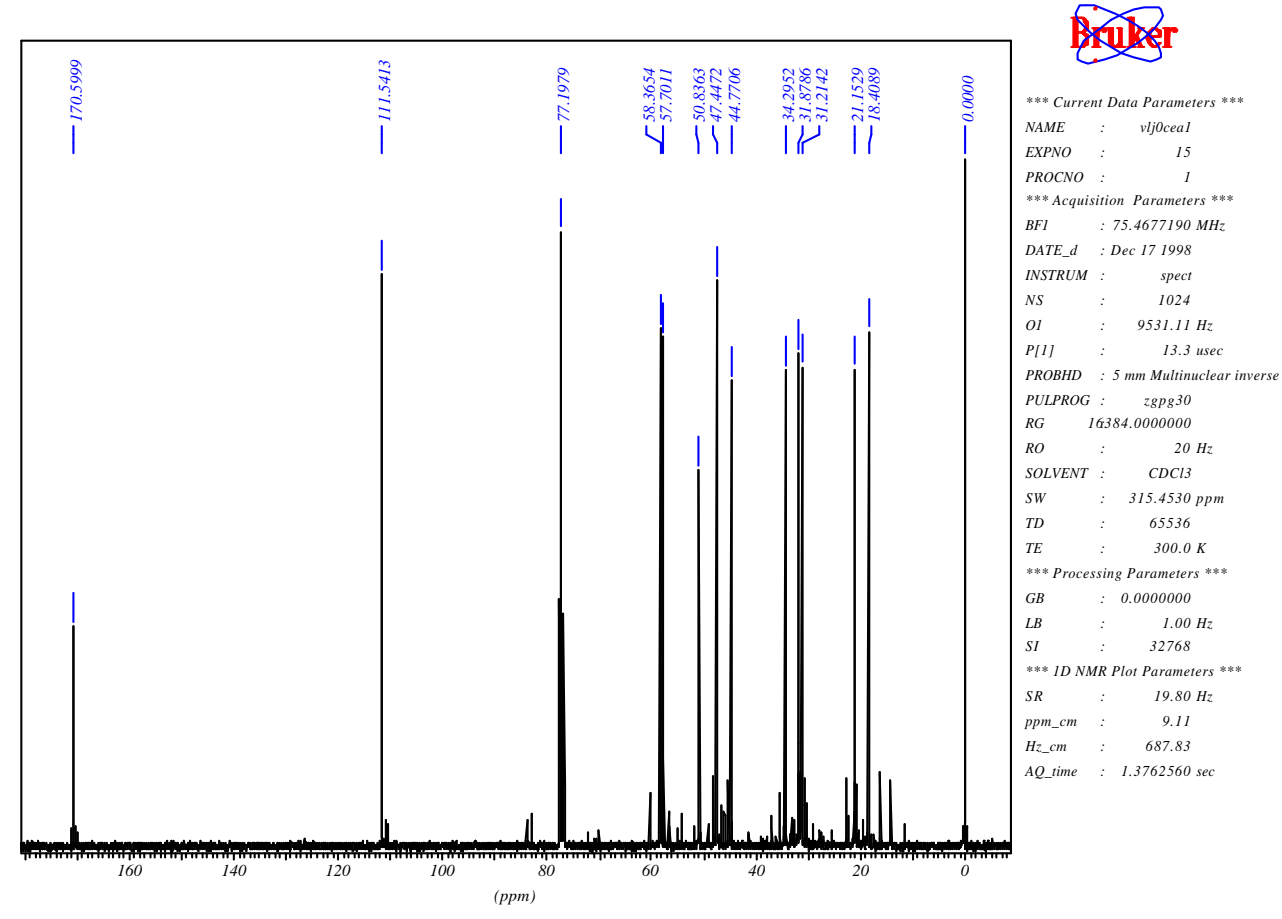

\section{Espectro de ${ }^{13} \mathrm{C}$-RMN (DEPT-135) do composto (6b)}

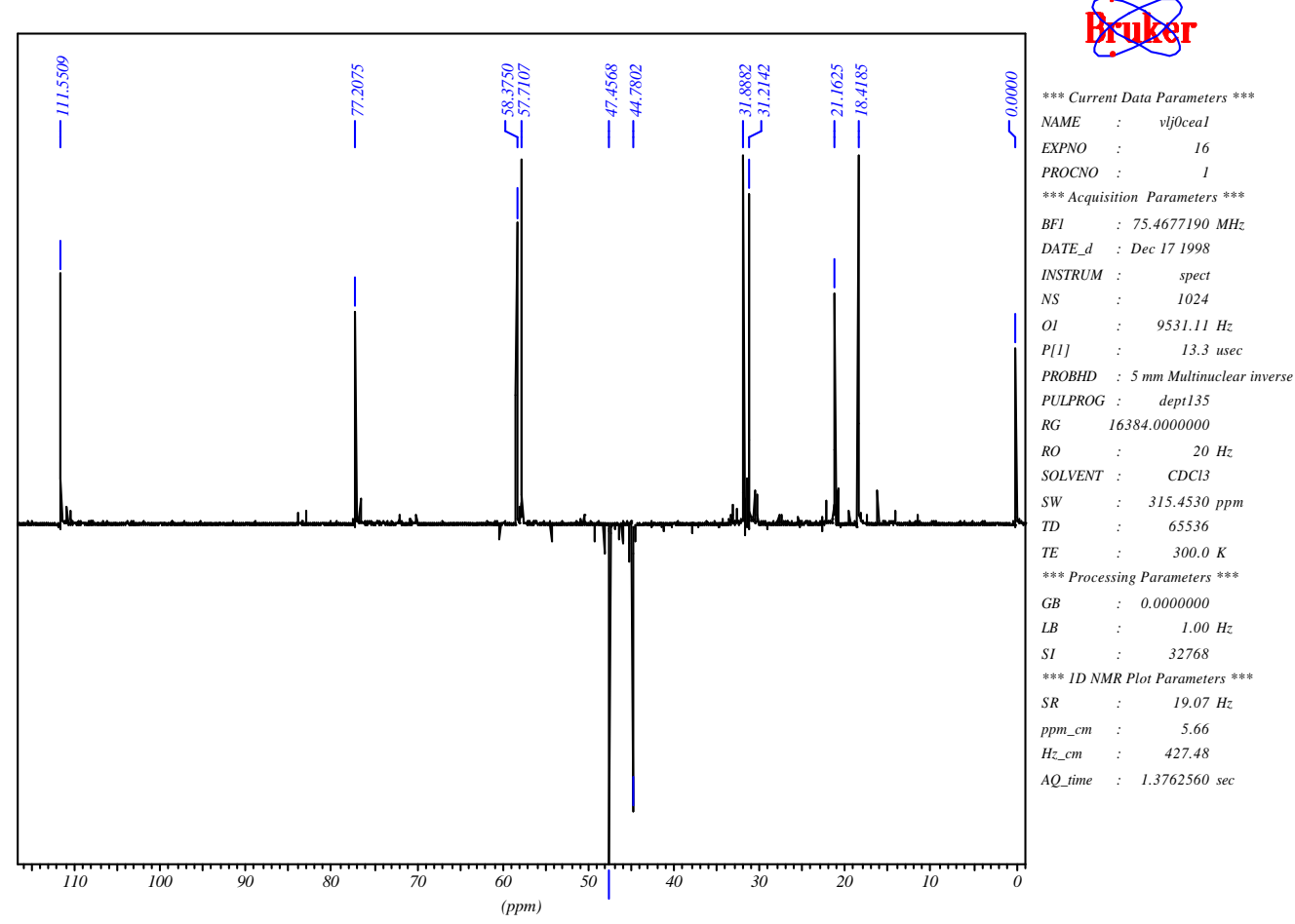




\section{Espectro HMQC (2D H/C) do composto (6b)}

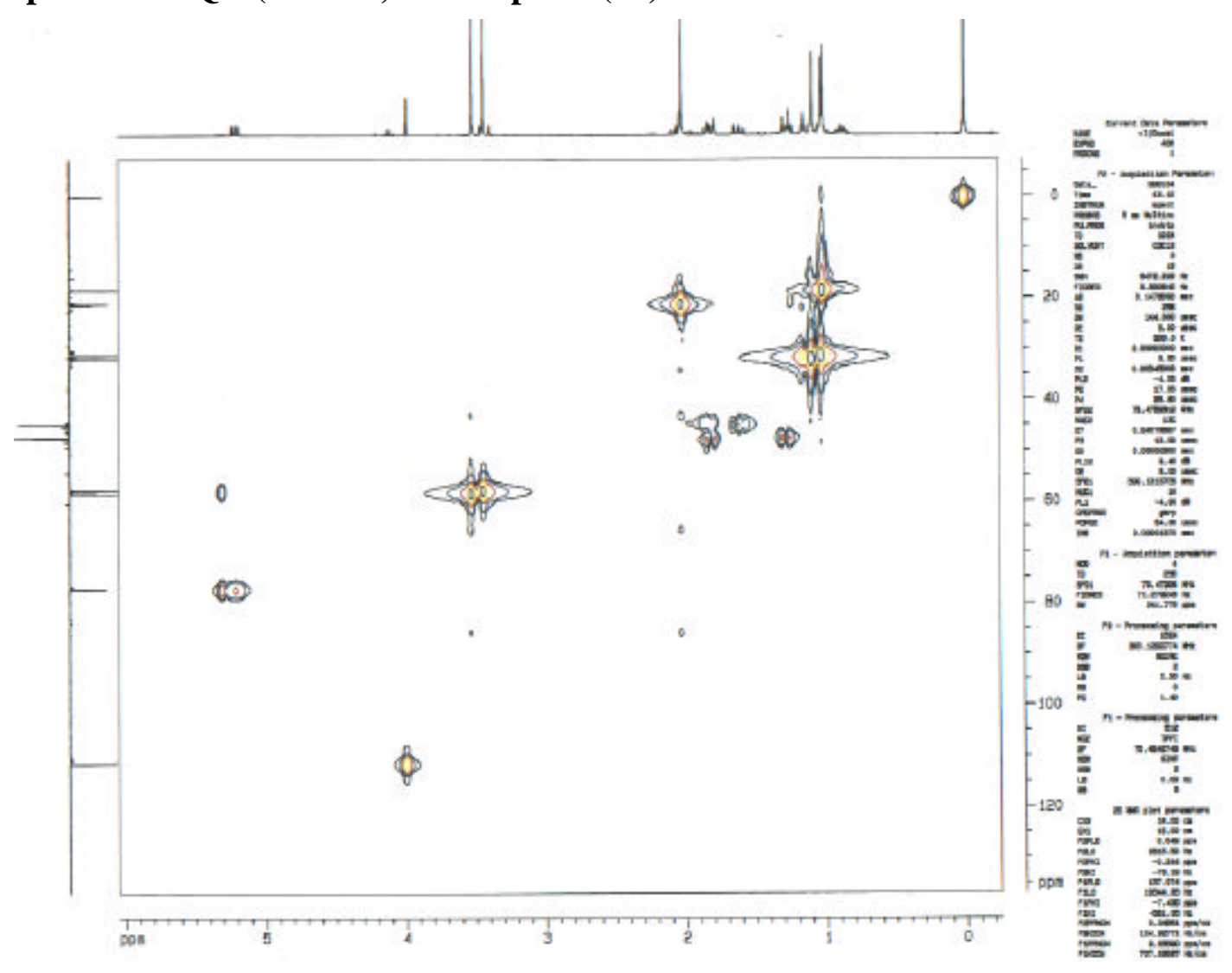

Espectro Jres do composto (6b)
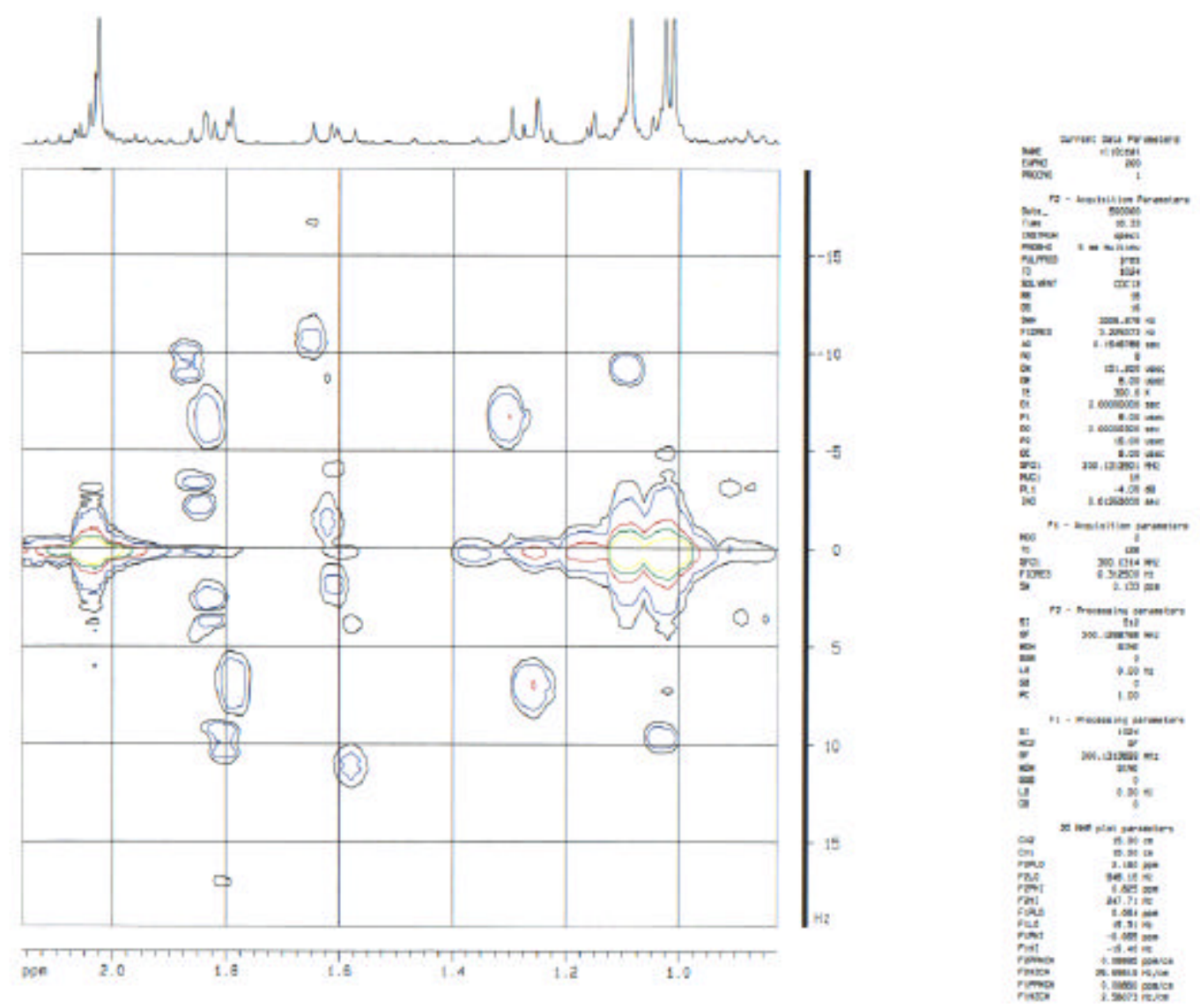


\section{Espectros NOE DIFF do composto (6b)}

$>$ irradiação na metila 6

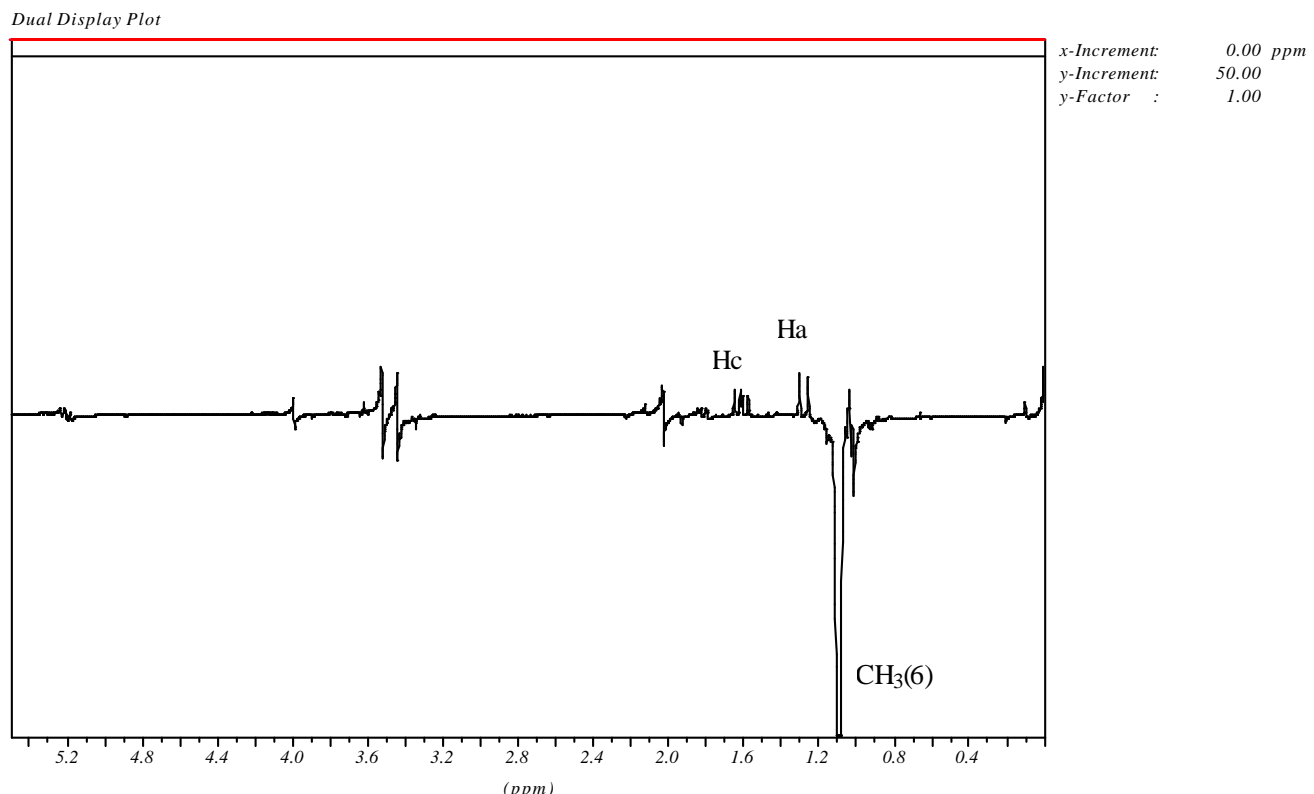

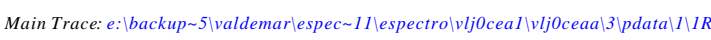
Second Trace: $\backslash$ WINID \SPC\ASPX32\VLJOCEAA\006001.1R

> irradiação na metila 7

Dual Display Plot

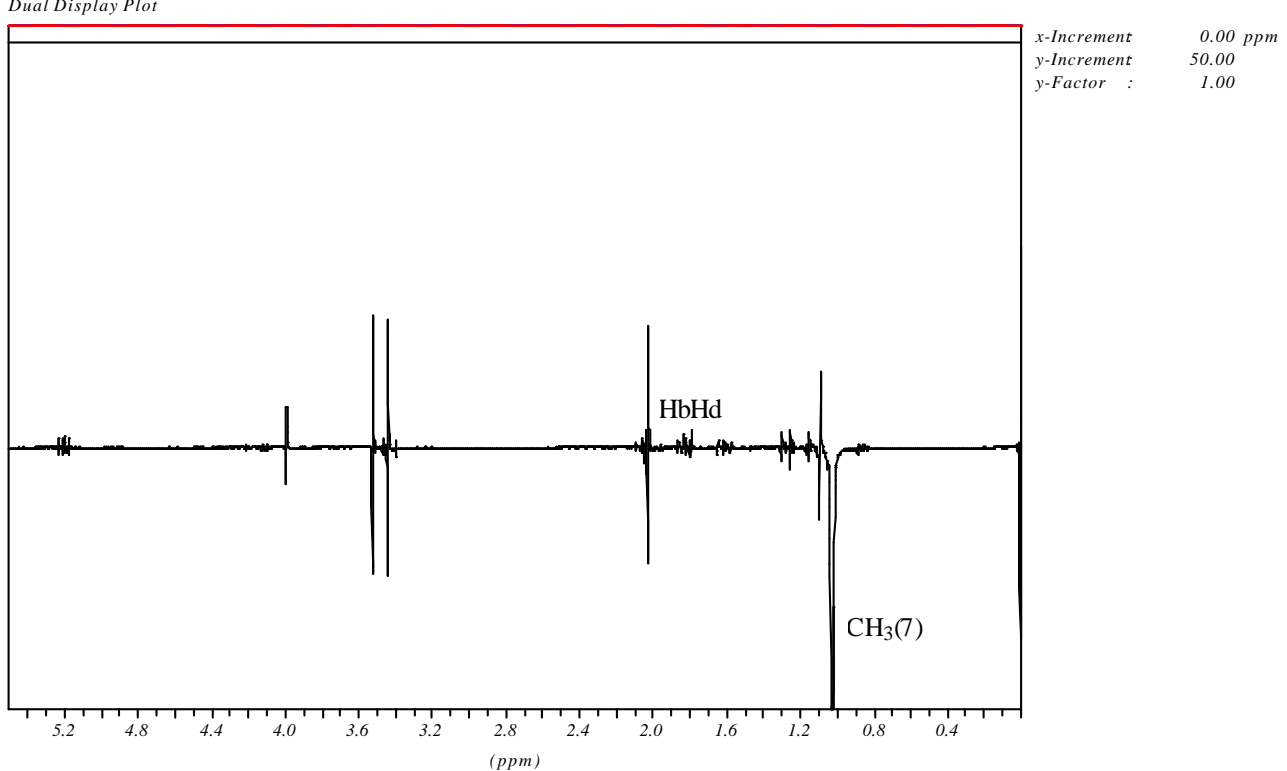

Main Trace: e:\backup $\sim 5 \backslash$ valdemarlespec $\sim 1 \backslash$ espectro\vljocea $\backslash$ vljoceaa $\backslash 4 \backslash p d a t a \backslash \backslash \backslash R$

Second Trace: $\backslash$ WINID $\backslash S P C \backslash A S P X 32 \backslash V L J O C E A A \backslash 006001.1 R$ 
irradiação na metila 8

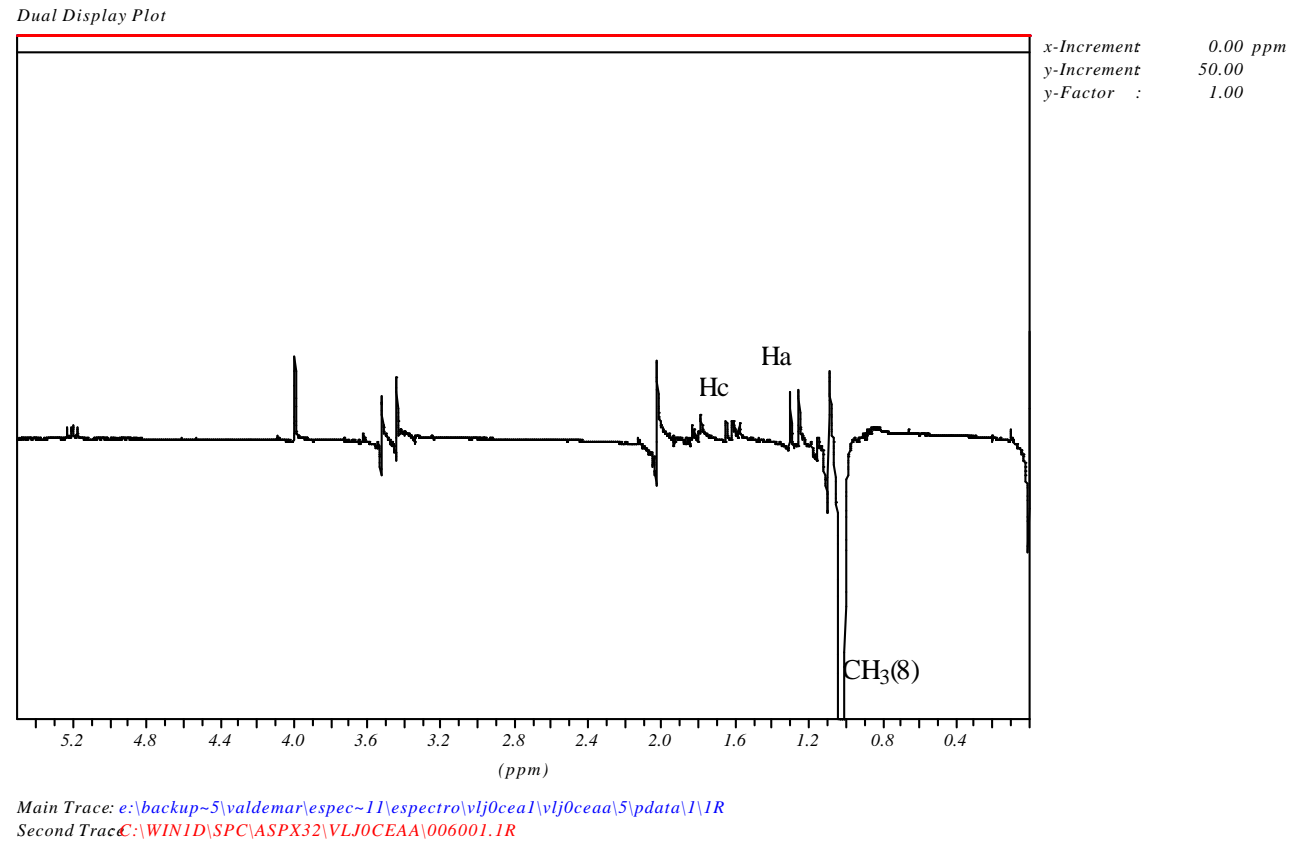

Tabela 20 - Dados espectrais de NOE DIFF do composto $\mathbf{6 b}\left(\mathrm{CDCl}_{3}\right)$.

\begin{tabular}{c|c|c}
\hline$\delta(\mathbf{p p m})$ Irradiado & Atribuição & Efeito NOE Observado \\
\hline 1,08 & & $\mathrm{Ha}, \mathrm{Hc}$ \\
1,02 & $\mathrm{CH}_{3}(6)$ & $\mathrm{Hb}, \mathrm{Hd}$ \\
1,01 & $\mathrm{CH}_{3}(7)$ & $\mathrm{Ha}, \mathrm{Hc}$ \\
\hline
\end{tabular}

Tabela 21 - Dados espectrais de ${ }^{1} \mathrm{H}$ e ${ }^{13} \mathrm{C}$ RMN para o $( \pm)$ - trans - 1 -Dimetoximetil2- acetoxi - 1,4,4 - trimetil-ciclopentano $\left(\mathrm{CDCl}_{3}\right)$

\begin{tabular}{|c|c|c|}
\hline C-, H- & ${ }^{1} \mathrm{H}(300 \mathrm{MHz}) \delta(\mathrm{ppm})$ & ${ }^{{ }^{13} \mathrm{C}}(75 \mathrm{MHz})$ \\
\hline 1 & $\mathrm{He}-5,2(\mathrm{dd}, 1 \mathrm{H}, \mathrm{Jec}=9,3$ e Jed=6,9 Hz) & 77,9 \\
\hline 2 & $\begin{array}{c}\text { Hd-1,83 (ddd, } 1 \mathrm{H}, \mathrm{Jdc}=12,4 ; \mathrm{Jde}=6,9 \text { e } \mathrm{Jdb}=1,3 \mathrm{~Hz}) \text { e Hc-1,61 } \\
(\mathrm{ddd}, 1 \mathrm{H}, \mathrm{Jcd}=12,4 ; \mathrm{Jce}=9,3 \text { e Jca }=0,5 \mathrm{~Hz})\end{array}$ & 44,8 \\
\hline 3 & & 34,3 \\
\hline 4 & $\begin{array}{c}\mathrm{Hb}-1,81(\mathrm{dd}, 1 \mathrm{H}, \mathrm{Jba}=13,7 \text { e } \mathrm{Jbd}=1,3 \mathrm{~Hz}) \text { e } \mathrm{Ha}-1,27(\mathrm{dd}, 1 \mathrm{H}, \\
\mathrm{Jab}=13,7 \text { e } \mathrm{Jac}=0,5 \mathrm{~Hz})\end{array}$ & 47,4 \\
\hline 5 & & 50,8 \\
\hline 6 & $1,08(\mathrm{~s}, 3 \mathrm{H})$ & 31,8 \\
\hline 7 & $1,02(\mathrm{~s}, 3 \mathrm{H})$ & 31,2 \\
\hline 8 & $1,01(\mathrm{~s}, 3 \mathrm{H})$ & 18,4 \\
\hline 9 & $3,99(\mathrm{~s}, 1 \mathrm{H})$ & 111,5 \\
\hline 10 & $3,52(\mathrm{~s}, 3 \mathrm{H})$ & 58,3 \\
\hline 11 & $3,44(\mathrm{~s}, 3 \mathrm{H})$ & 57,7 \\
\hline 12 & & 170,6 \\
\hline 13 & $2,02(\mathrm{~s}, 3 \mathrm{H})$ & 21,1 \\
\hline
\end{tabular}




\section{Composto (7a)}

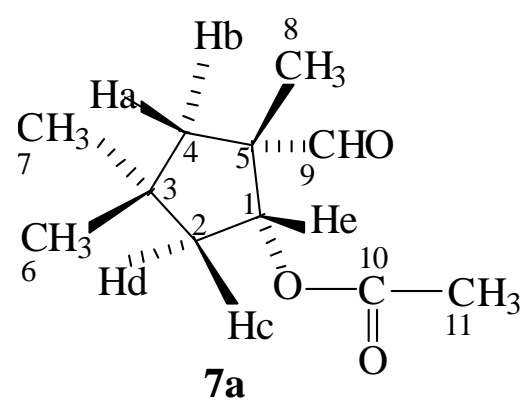

Espectro de ${ }^{1}$ H-RMN do composto (7a)

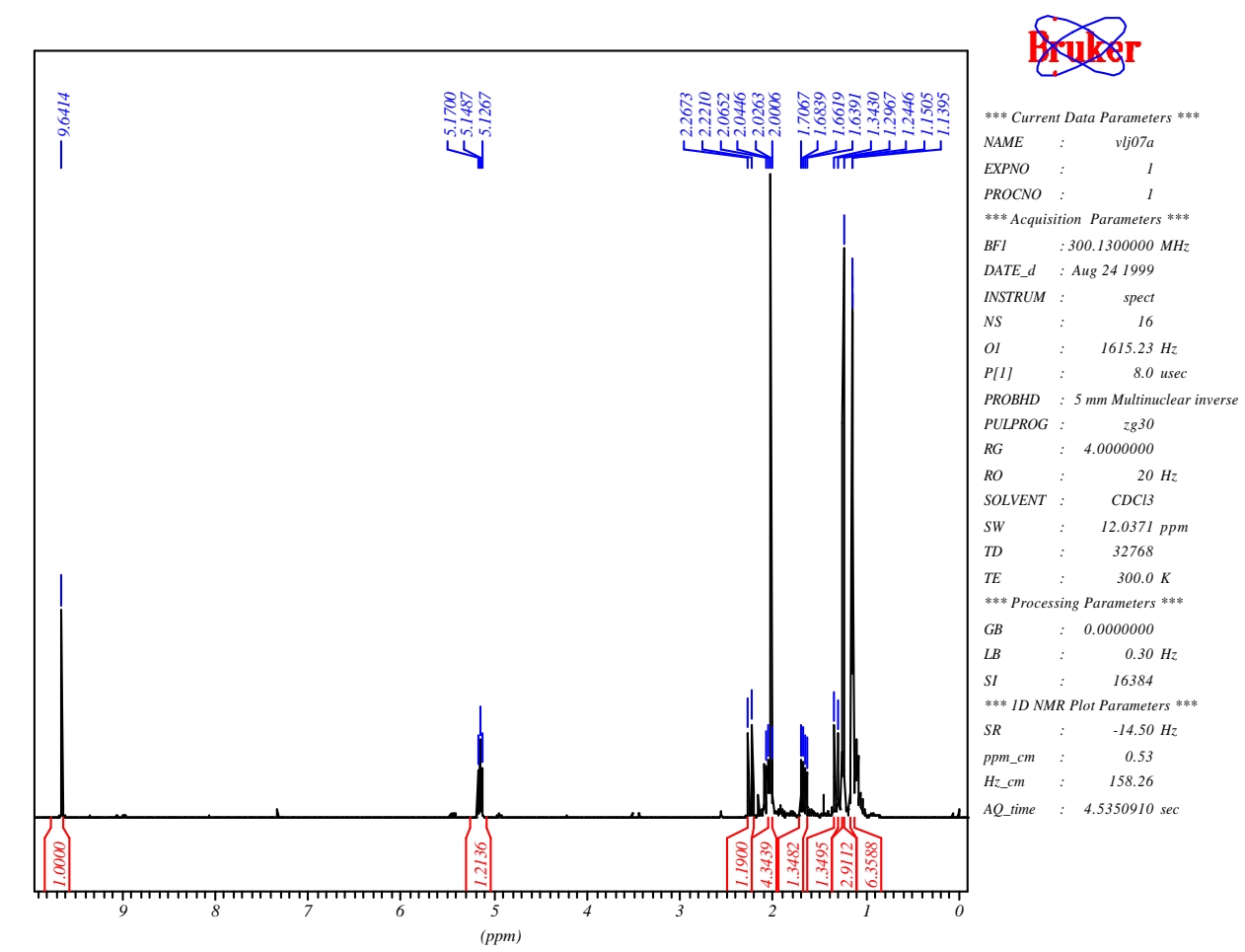

Tabela 22 - Dados espectrais de ${ }^{1} \mathrm{H}$ RMN do composto 7a $\left(\mathrm{CDCl}_{3}\right)$.

\begin{tabular}{c|l|c|c}
\hline$\delta(\mathbf{p p m})$ & Atribuição & sinal & J (Hz) \\
\hline 9,64 & $(9)$ & $\mathrm{s}$ & \\
5,15 & $\mathrm{He}$ & $\mathrm{t}$ & $\mathrm{Jec}=\mathrm{Jed}=6,5 \mathrm{~Hz}$ \\
2,24 & $\mathrm{Hb}$ & $\mathrm{d}$ & $\mathrm{Jba}=13,9 \mathrm{~Hz}$ \\
2,03 & $\mathrm{CH}_{3}(11)$ & $\mathrm{s}$ & $\mathrm{Jcd}=13,5 ; \mathrm{Jce}=6,5 \mathrm{e}$ \\
2,03 & $\mathrm{Hc}$ & $\mathrm{ddd}$ & $\mathrm{Jca}=2,6 \mathrm{~Hz}$ \\
& & $\mathrm{dd}$ & $\mathrm{Jdc}=13,5 \mathrm{e} \mathrm{Jde}=6,5 \mathrm{~Hz}$ \\
1,67 & $\mathrm{Hd}$ & $\mathrm{dd}$ & $\mathrm{Jab}=13,9 \mathrm{e} \mathrm{Jac}=2,6 \mathrm{~Hz}$ \\
1,32 & $\mathrm{Ha}$ & $\mathrm{s}$ & \\
1,24 & $\mathrm{CH}_{3}(6)$ & $\mathrm{s}$ & \\
1,15 & $\mathrm{CH}_{3}(8)$ & $\mathrm{s}$ & \\
1,13 & $\mathrm{CH}_{3}(7)$ & & \\
\hline
\end{tabular}




\section{Espectro de ${ }^{13}$ C-RMN (BB) do composto (7a)}

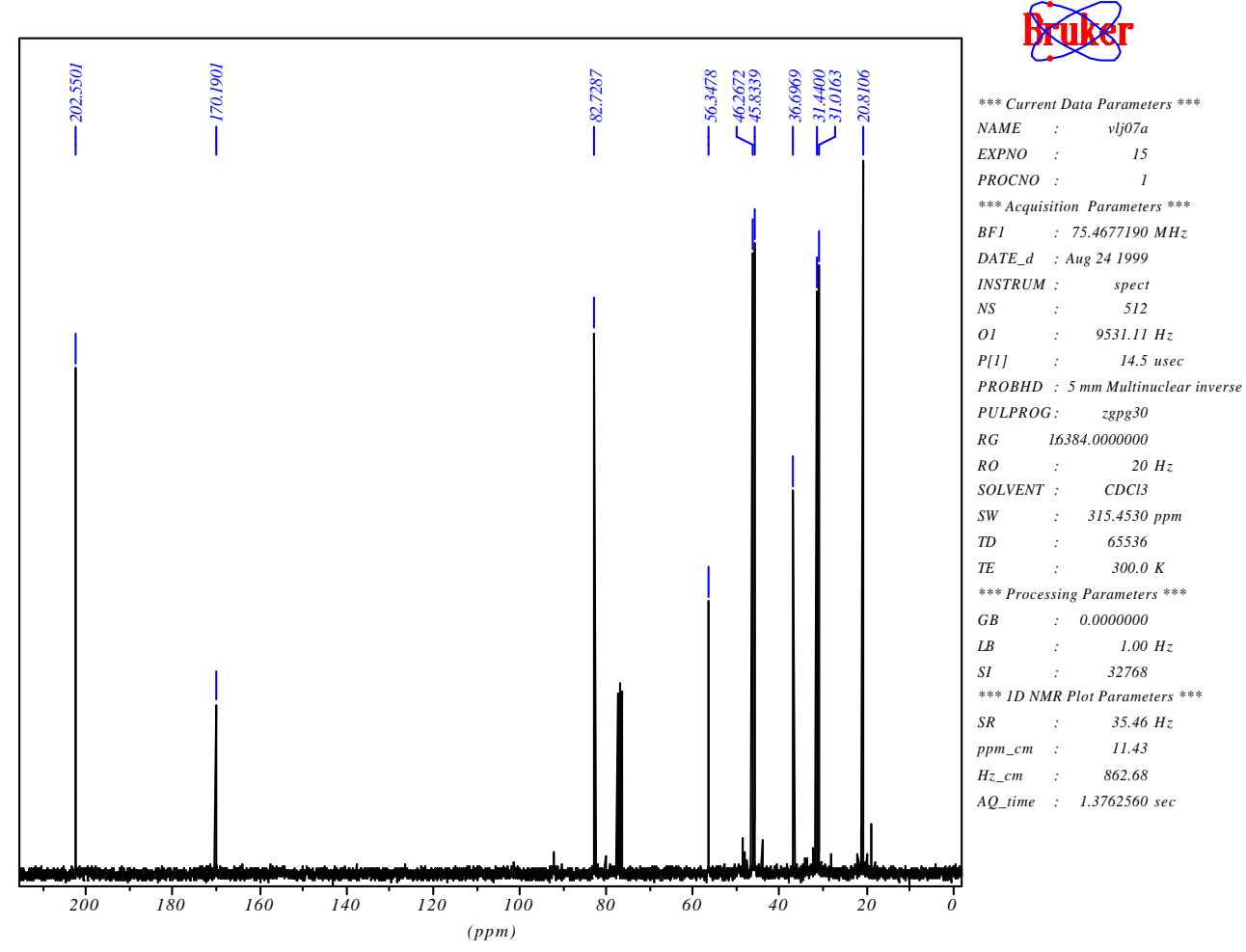

Espectro de ${ }^{13}$ C-RMN (DEPT-135) do composto (7a)

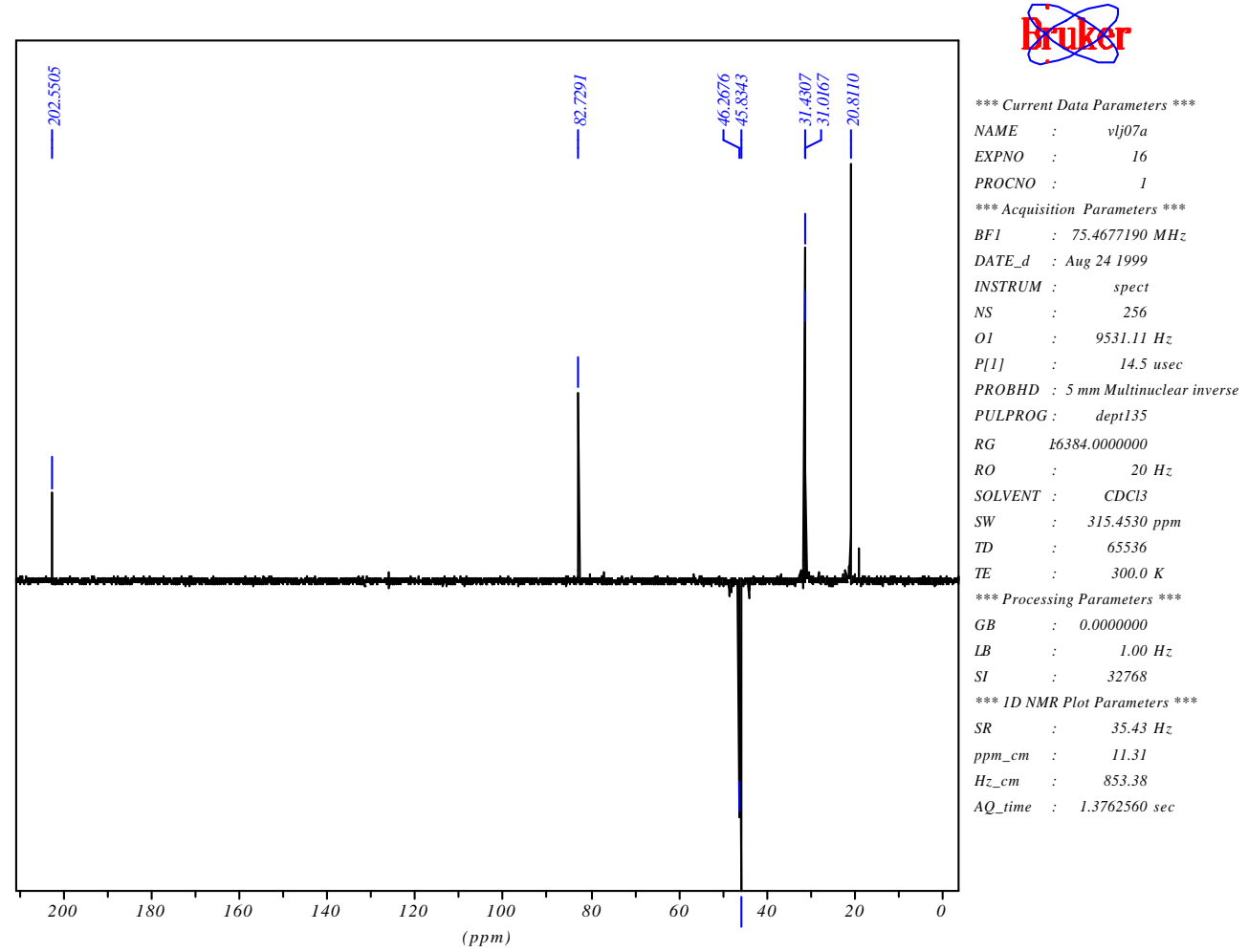




\section{Espectro HMQC (2D H/C) do composto (7a)}

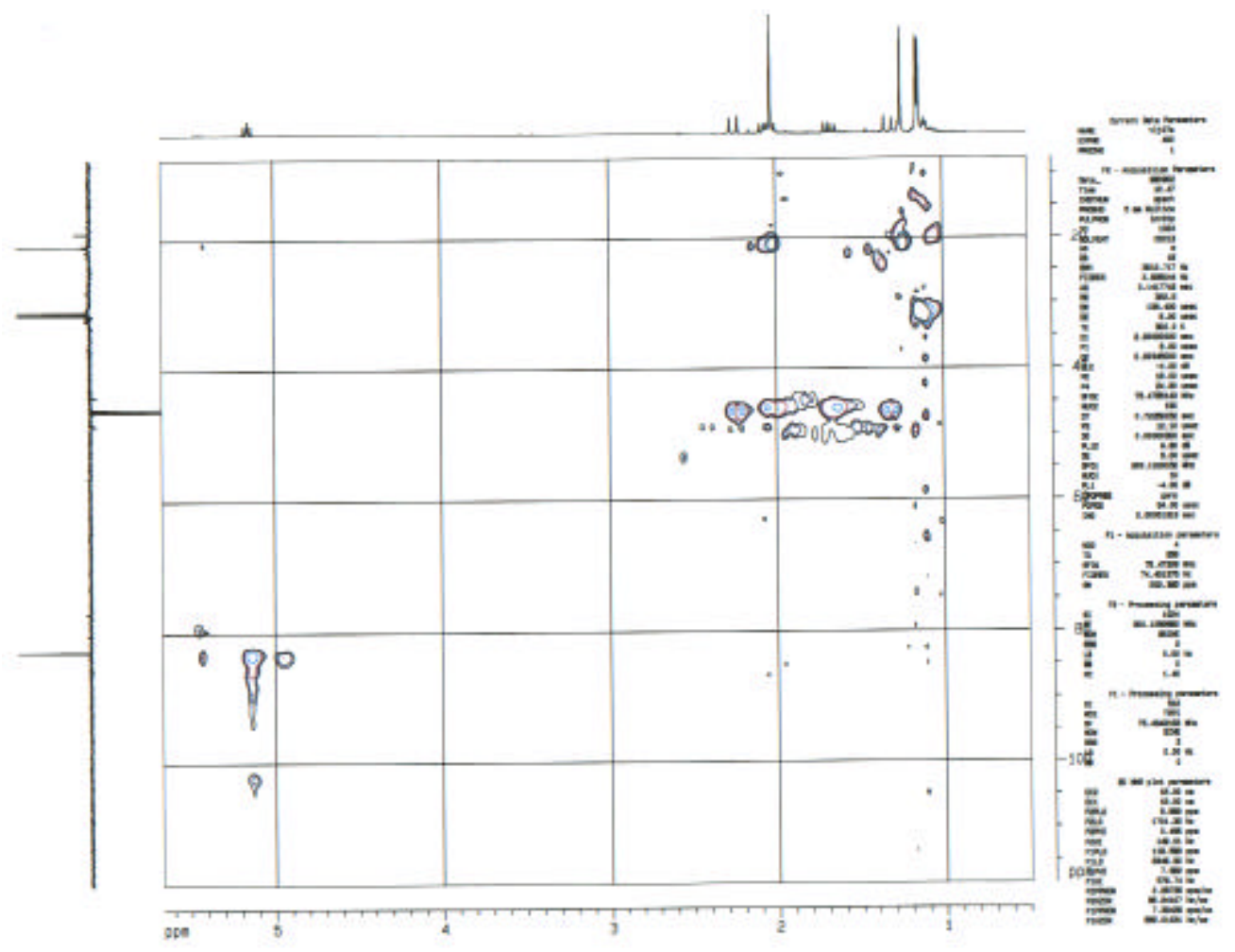

\section{Espectro Jres do composto (7a)}
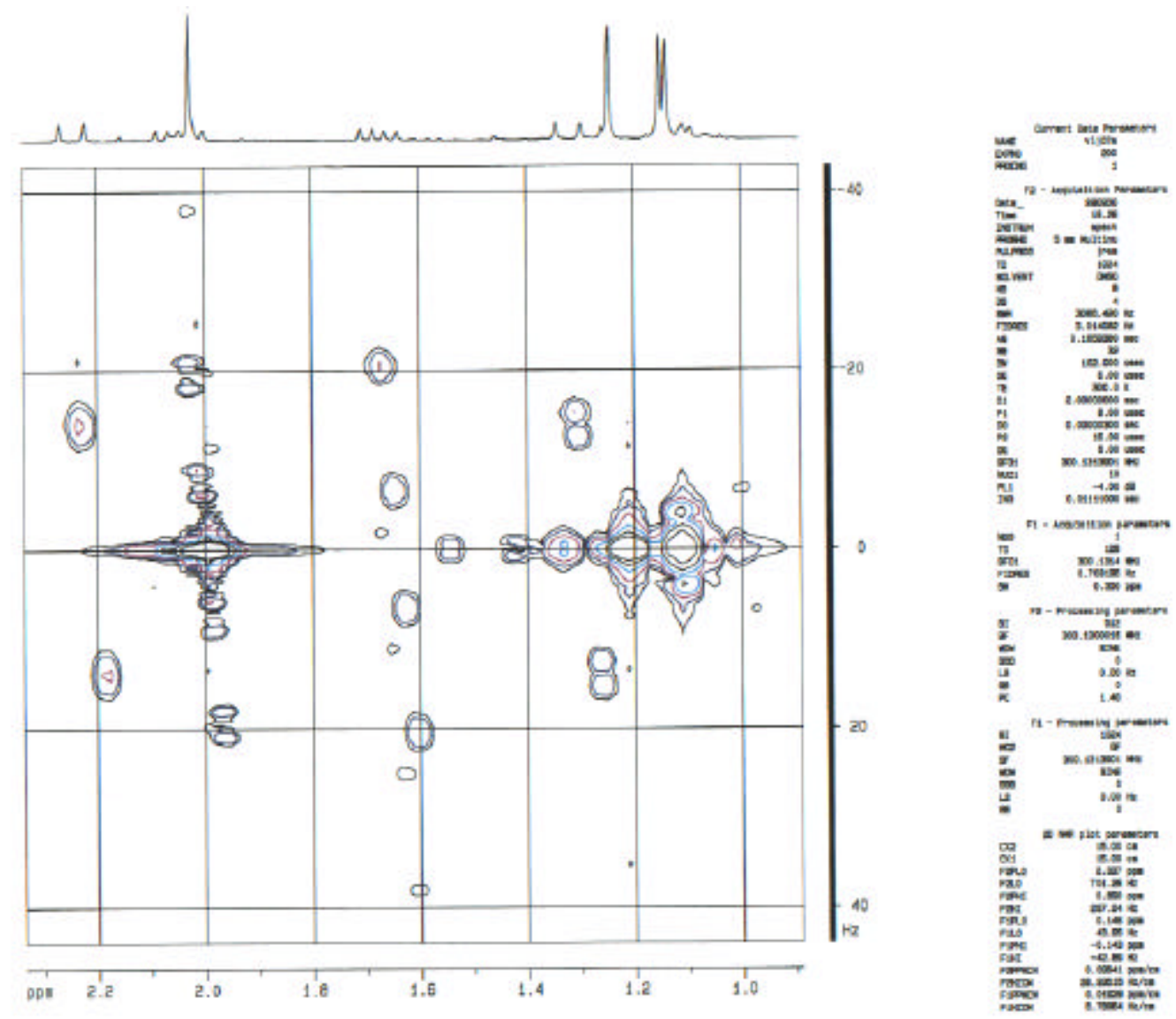


\section{Espectros NOE DIFF do composto (7a)}

$>$ irradiação na metila 6

Dual Display Plot

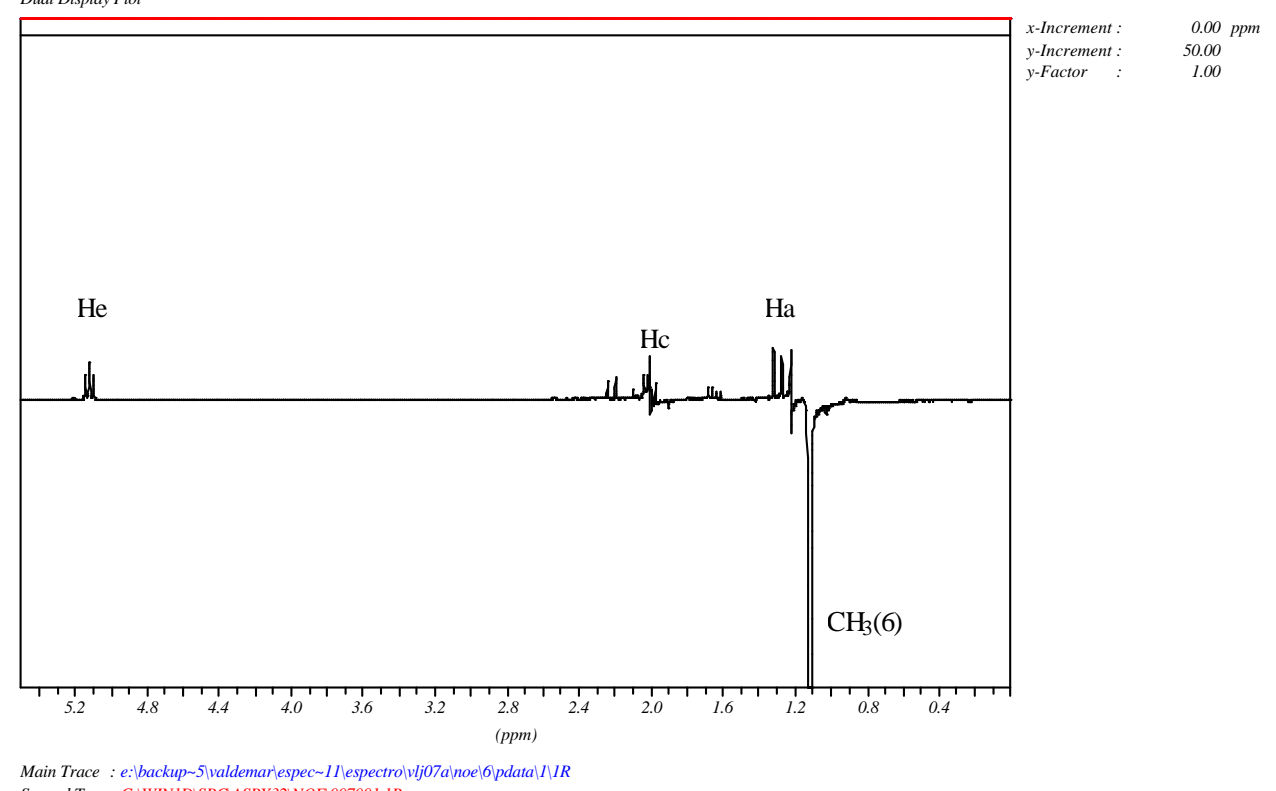

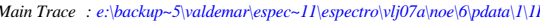

Second Trace C:IWINIDISPCASPX32WOEV007001.1R

irradiação na metila 7

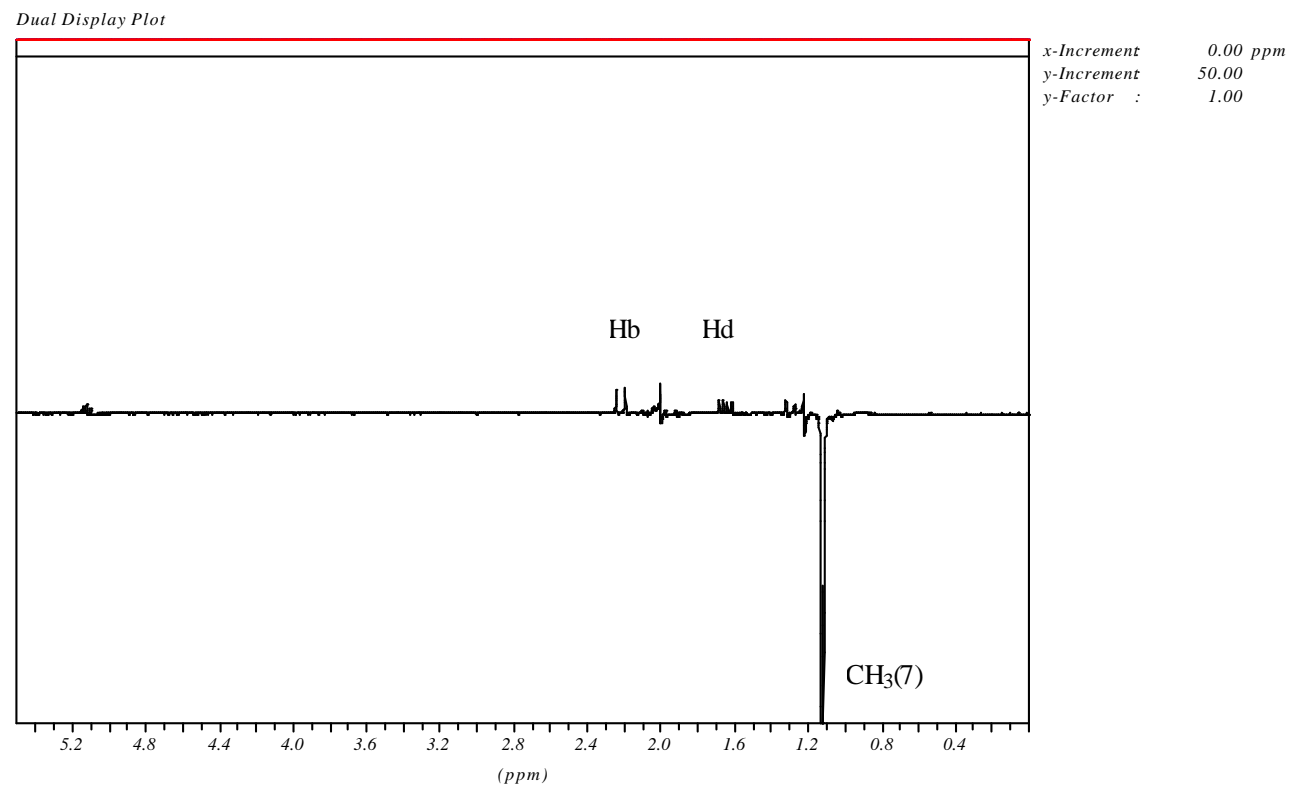

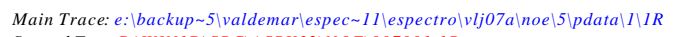

Second TraceC: $\backslash$ WINID $\backslash S P C \backslash A S P X 32 \backslash N O E \backslash 007001.1 R$

Aplicações de Técnicas de RMN À Determinação Estrutural de Intermediários Sintéticos 
irradiação a metila 8

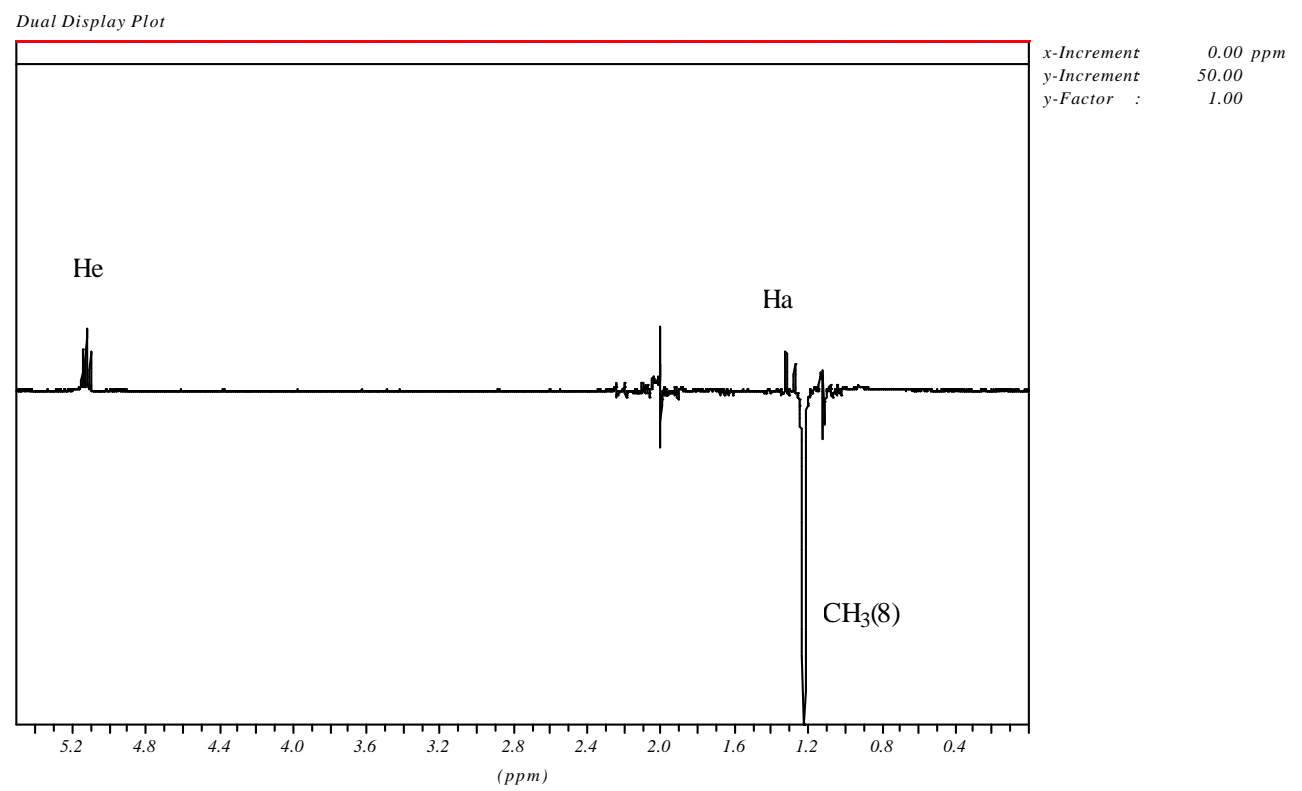

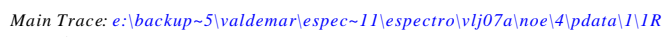

Second Trace: $\backslash W I N 1 D \backslash S P C \backslash A S P X 32 \backslash N O E \backslash 007001.1 R$

Tabela 23 - Dados espectrais de NOE DIFF do composto 7a $\left(\mathrm{CDCl}_{3}\right)$.

\begin{tabular}{c|c|c}
\hline$\delta$ (ppm)Irradiado & Atribuição & Efeito NOE Observado \\
& & \\
1,08 & $\mathrm{CH}_{3}(8)$ & $\mathrm{Ha}, \mathrm{He}$ \\
1,02 & $\mathrm{CH}_{3}(7)$ & $\mathrm{Hb}, \mathrm{Hd}$ \\
1.01 & $\mathrm{CH}_{3}(6)$ & $\mathrm{Ha}, \mathrm{Hc}, \mathrm{He}$ \\
\hline
\end{tabular}

Tabela 24 - Dados espectrais de ${ }^{1} \mathrm{H}$ e ${ }^{13} \mathrm{C}$ RMN para o $( \pm)$ - cis - 2-acetoxi - 1,4,4 trimetil-ciclopentano-1-carboxaldeído $\left(\mathrm{CDCl}_{3}\right)$

\begin{tabular}{|c|c|c|}
\hline $\mathrm{C}-, \mathrm{H}-$ & ${ }^{1} \mathrm{H}(300 \mathrm{MHz}) \delta(\mathrm{ppm})$ & ${ }^{13} \mathrm{C}(75 \mathrm{MHz})$ \\
\hline 1 & $\mathrm{He}-5,15(\mathrm{t}, 1 \mathrm{H}, \mathrm{Jec}=\mathrm{Jed}=6,5 \mathrm{~Hz})$ & 82,72 \\
\hline 2 & $\begin{array}{c}\mathrm{Hc}-2,03(\mathrm{ddd}, 1 \mathrm{H}, \mathrm{Jcd}=13,5 ; \mathrm{Jce}=6,5 \mathrm{e} \mathrm{Jca}=2,6 \mathrm{~Hz}) \text { e Hd-1,67 } \\
(\mathrm{dd}, 1 \mathrm{H}, \mathrm{Jdc}=13,5 \text { e Jde=6,5 Hz) }\end{array}$ & 45,83 \\
\hline 3 & & 36,69 \\
\hline 4 & $\mathrm{Hb}-2,24(\mathrm{~d}, 1 \mathrm{H}, \mathrm{Jba}=13,9 \mathrm{~Hz})$ e Ha-1,32 (dd, 1H, Jab= 13,9 e \\
$\mathrm{Jac}=2,6 \mathrm{~Hz})$ & 46,26 \\
\hline 5 & & 56,34 \\
\hline 6 & $1,13(\mathrm{~s}, 3 \mathrm{H})$ & 31,44 \\
\hline 7 & $1,15(\mathrm{~s}, 3 \mathrm{H})$ & 31,01 \\
\hline 8 & $1,24(\mathrm{~s}, 3 \mathrm{H})$ & 20,81 \\
\hline 9 & $9,64(\mathrm{~s}, 1 \mathrm{H})$ & 202,55 \\
\hline 10 & & 170,19 \\
\hline 11 & $2,03(\mathrm{~s}, 3 \mathrm{H})$ & 20,81 \\
\hline
\end{tabular}




\section{Composto (7b)}

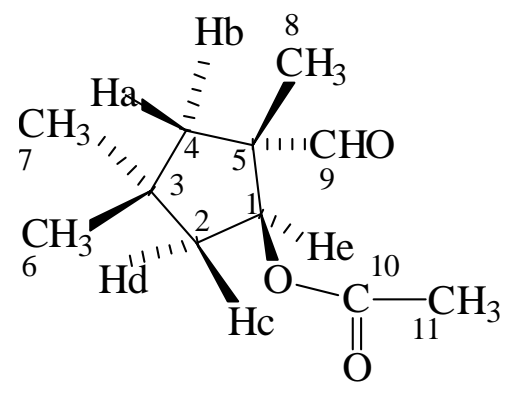

Espectro de ${ }^{1}$ H-RMN do composto $(7 b)$

7b

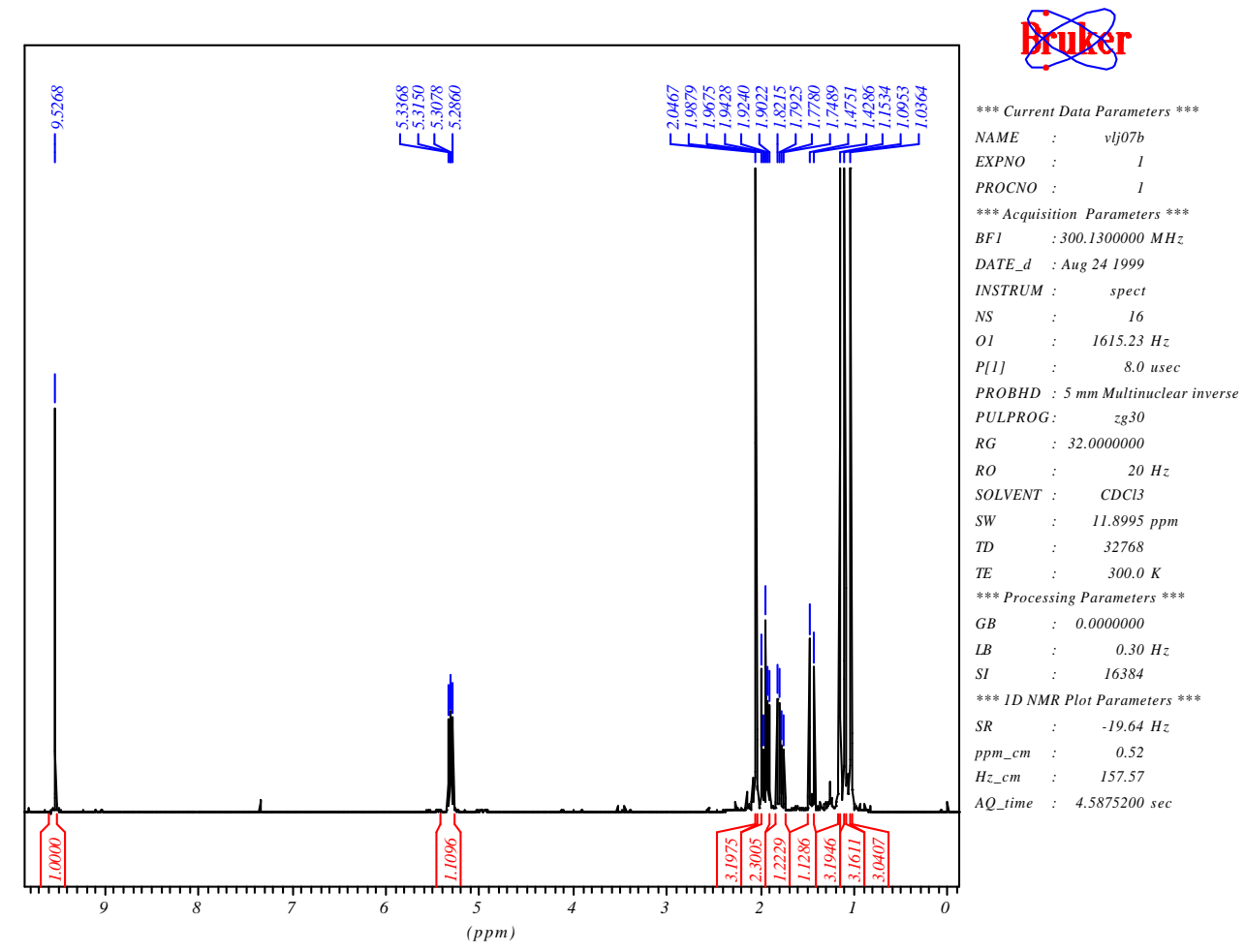

Tabela 25 - Dados espectrais de ${ }^{1} \mathrm{H}$ RMN do composto $7 \mathbf{b}\left(\mathrm{CDCl}_{3}\right)$.

\begin{tabular}{c|l|c|c}
\hline$\delta(\mathbf{p p m})$ & Atribuição & sinal & J $(\mathbf{H z})$ \\
\hline 9,52 & $(9)$ & $\mathrm{s}$ & \\
5,31 & $\mathrm{He}$ & $\mathrm{dd}$ & $\mathrm{Jec}=8,7 \mathrm{e} \mathrm{Jed}=6,5 \mathrm{~Hz}$ \\
2,04 & $\mathrm{CH}_{3}(11)$ & $\mathrm{s}$ & \\
1,97 & $\mathrm{Hb}$ & $\mathrm{dd}$ & $\mathrm{Jba}=13,9 \mathrm{e} \mathrm{Jbd}=1,2 \mathrm{~Hz}$ \\
1,93 & $\mathrm{Hd}$ & $\mathrm{ddd}$ & $\mathrm{Jdc}=13 ; \mathrm{Jde}=6,5 \mathrm{e}$ \\
& & & $\mathrm{Jdb}=1,2 \mathrm{~Hz}$ \\
1,78 & $\mathrm{Hc}$ & $\mathrm{dd}$ & $\mathrm{Jcd}=13 \mathrm{e} \mathrm{Jce}=8,7 \mathrm{~Hz}$ \\
1,45 & $\mathrm{Ha}$ & $\mathrm{d}$ & $\mathrm{Jab}=13,9 \mathrm{~Hz}$ \\
1,08 & $\mathrm{CH}_{3}(6)$ & $\mathrm{s}$ & \\
1,02 & $\mathrm{CH}_{3}(8)$ & $\mathrm{s}$ & \\
1,01 & $\mathrm{CH}_{3}(7)$ & $\mathrm{s}$ & \\
\hline
\end{tabular}




\section{Espectro de ${ }^{13}$ C-RMN (BB) do composto (7b)}

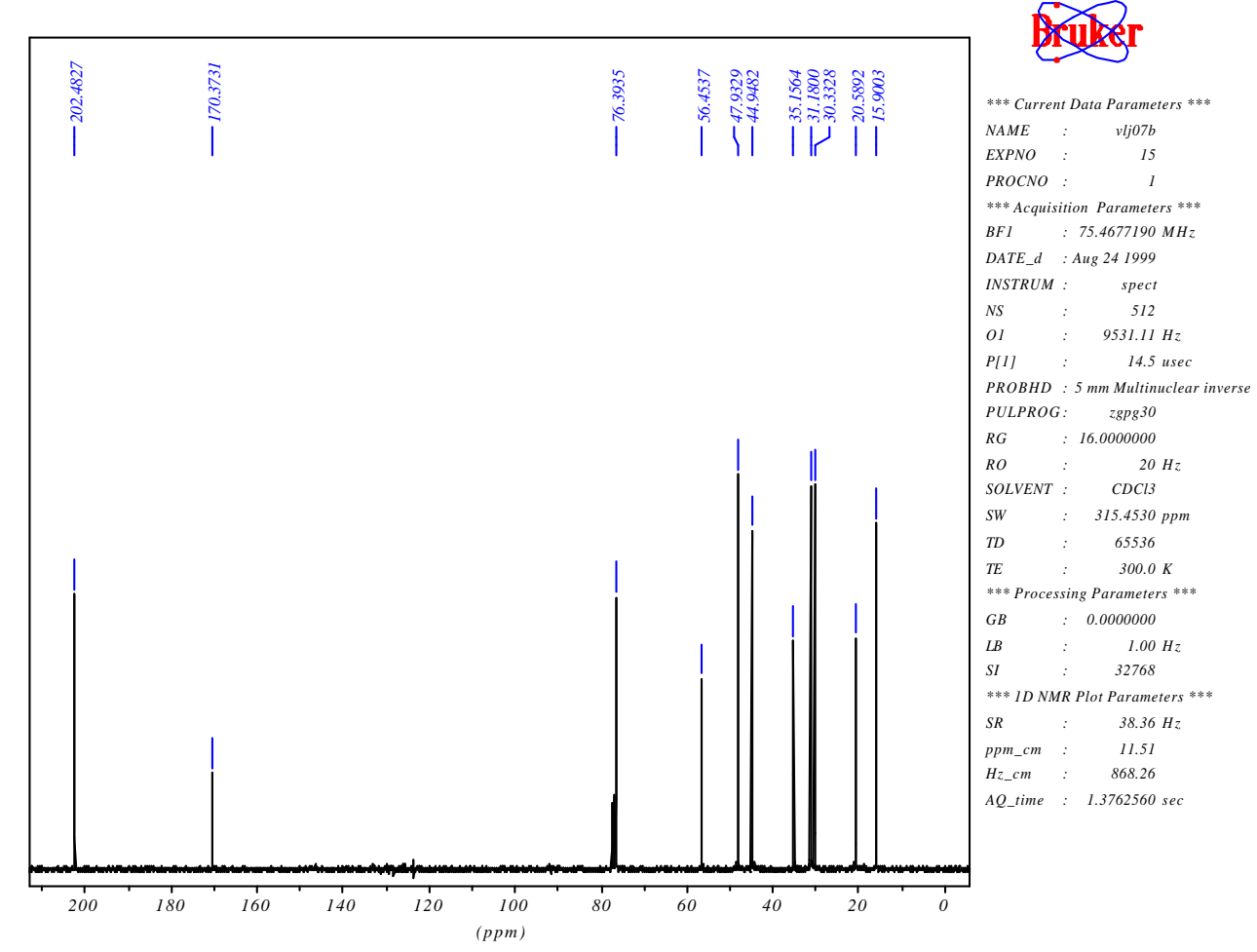

Espectro de ${ }^{13}$ C-RMN (DEPT-135) do composto (7b)

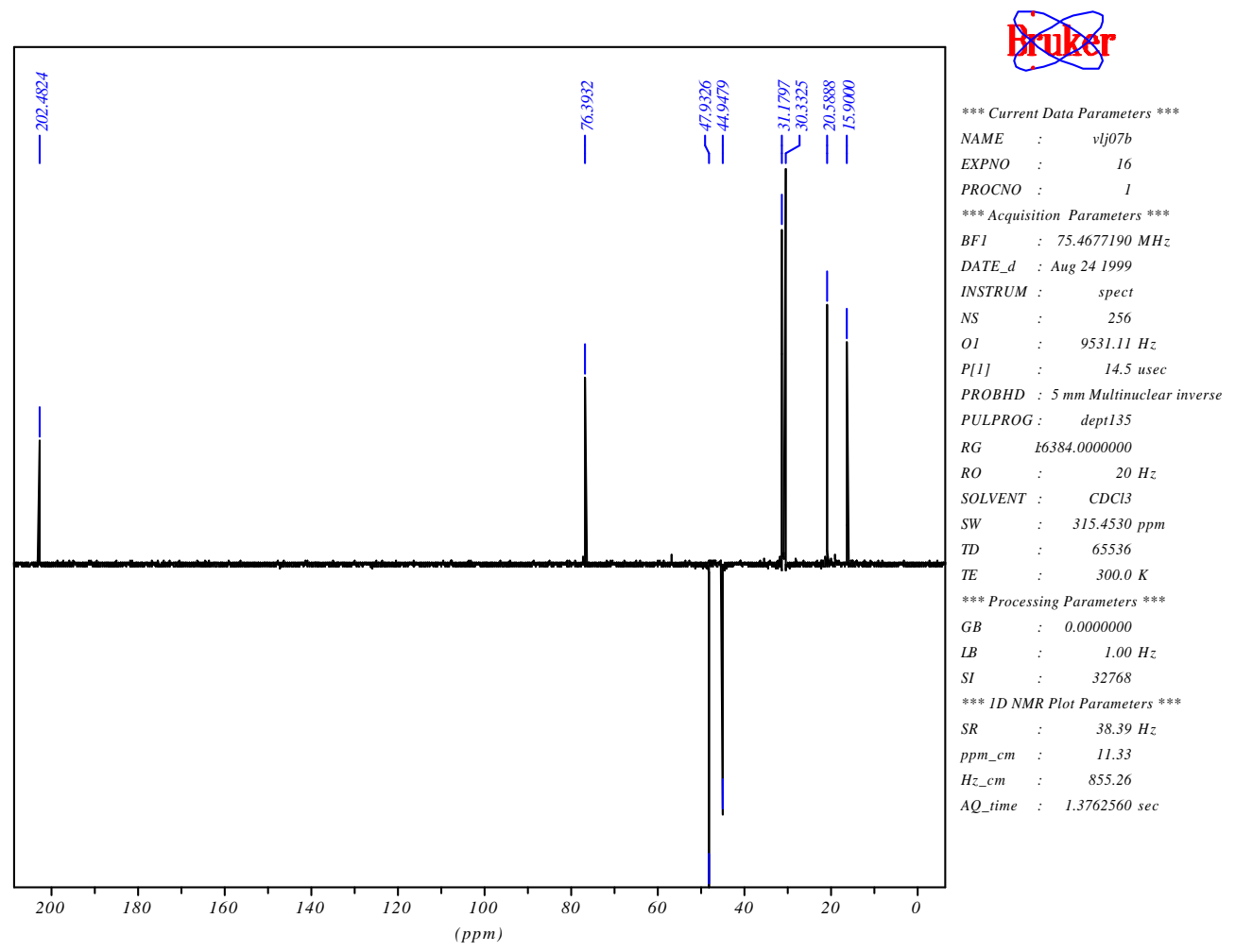




\section{Espectro HMQC (2D H/C) do composto (7b)}

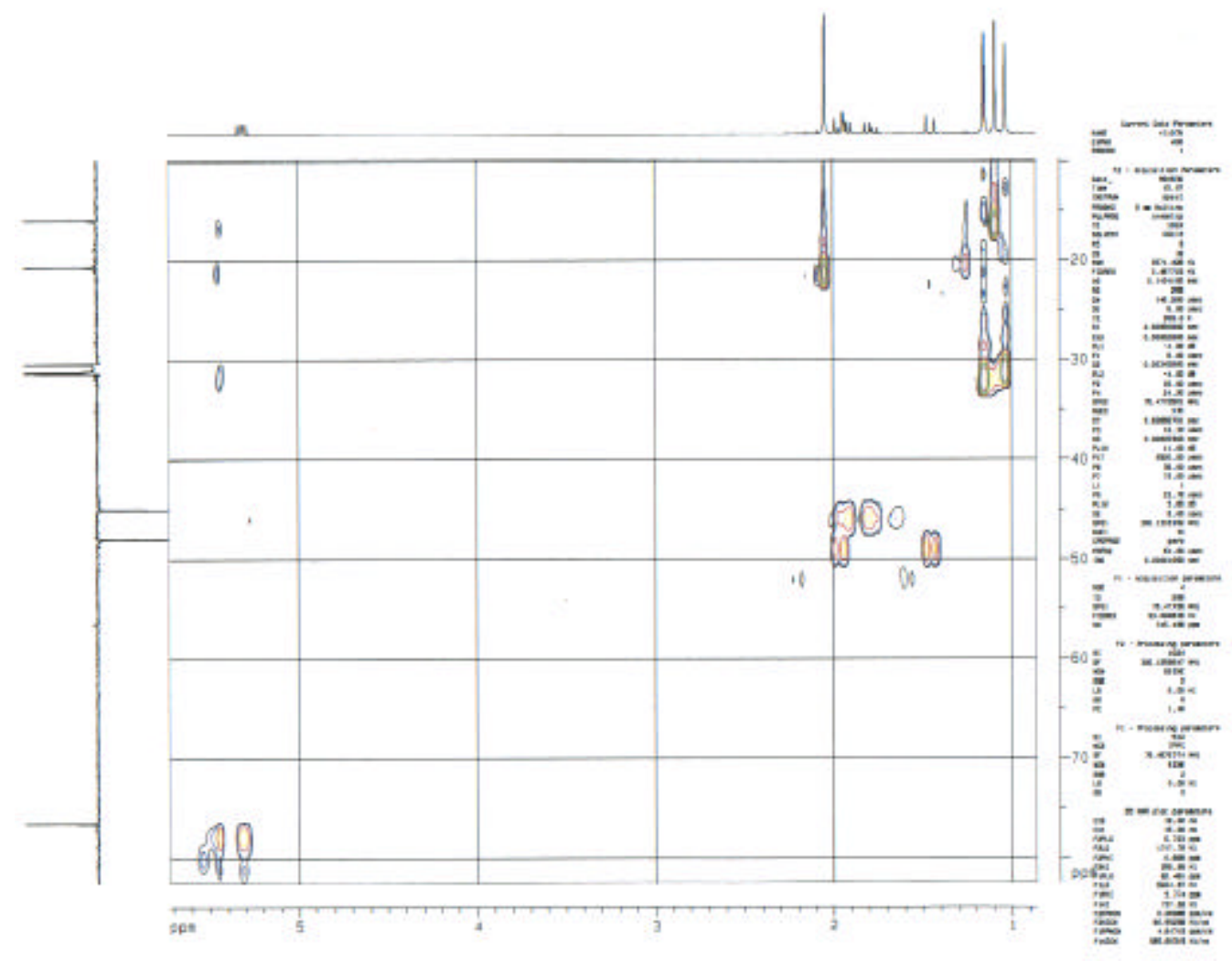

Espectro Jres do composto (7b)
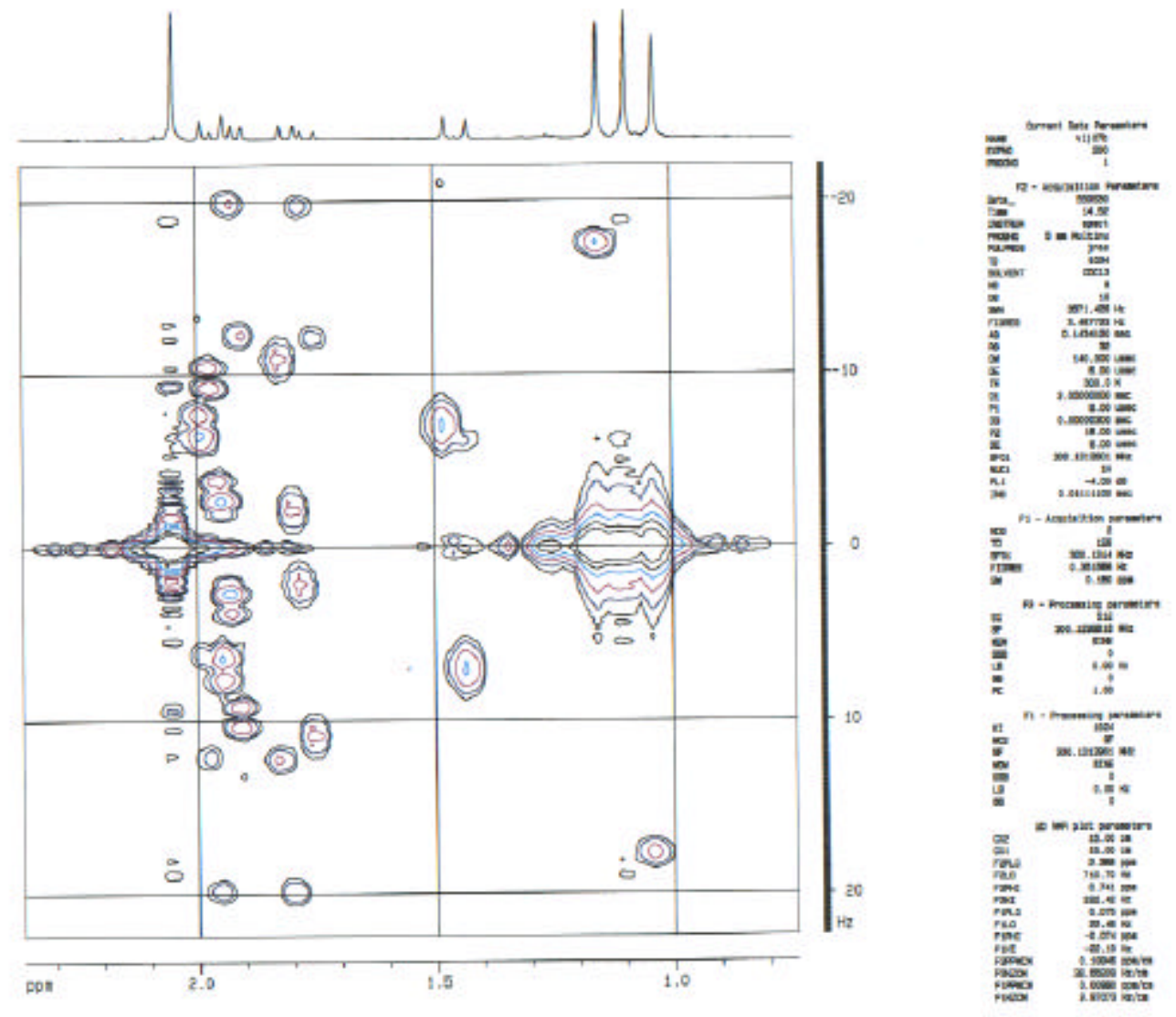


\section{Espectros NOE DIFF do composto (7b)}

$>$ irradiação na metila 6

Dual Display Plot

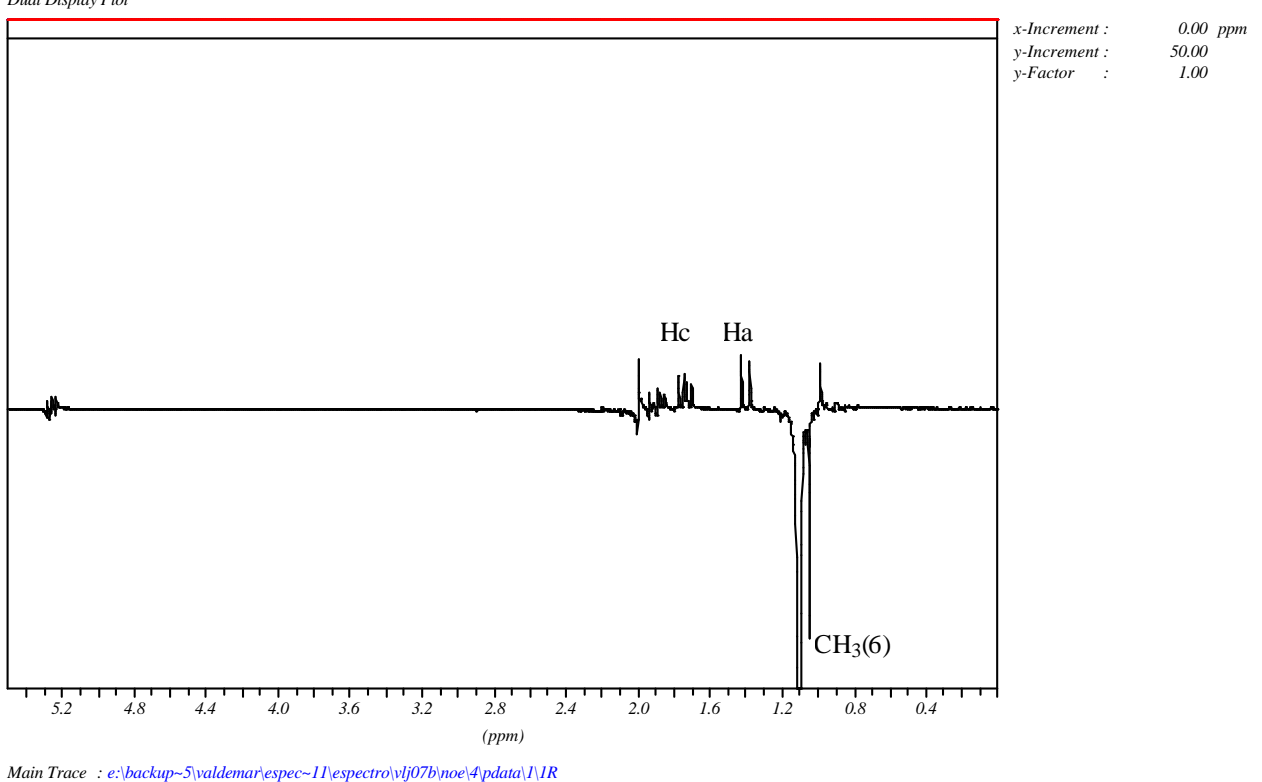

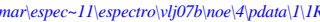

Second Trace C:IWINIDISPCASPX32WOEV007001.1R

irradiação na metila 7

Dual Display Plot

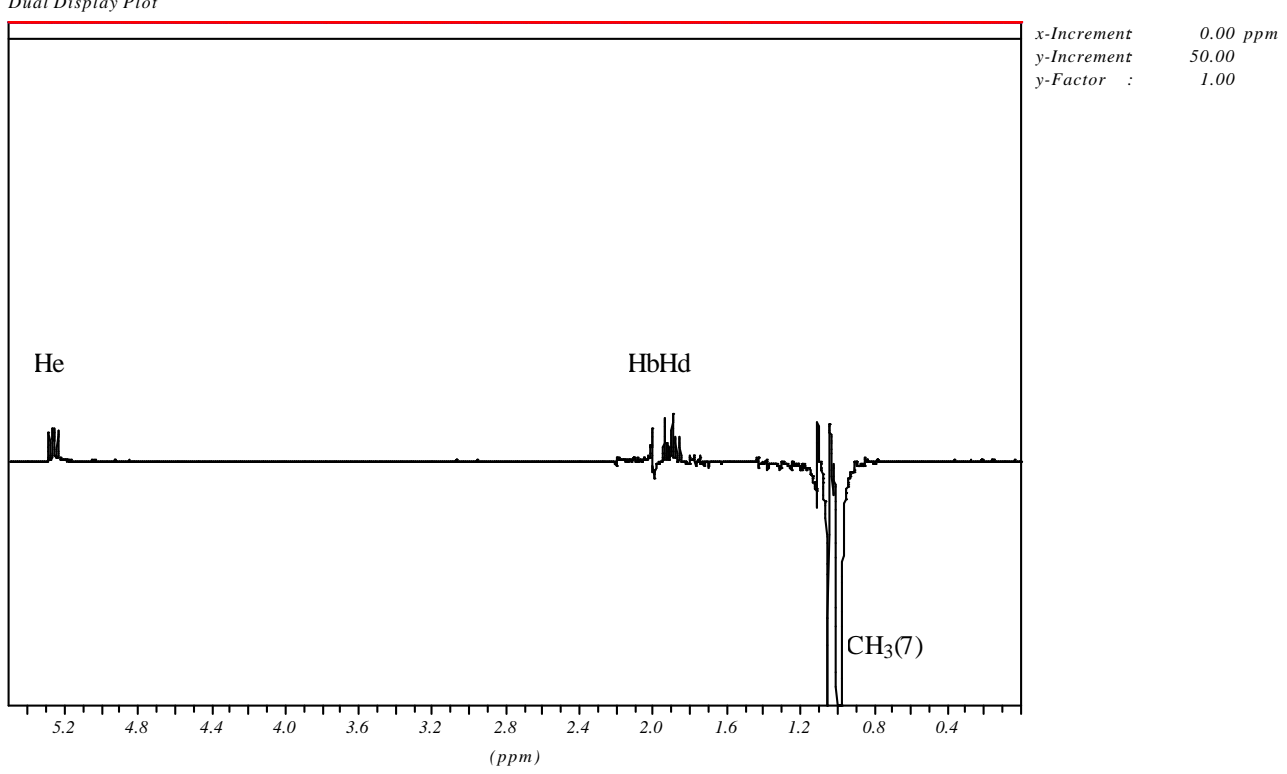

Main Trace: e:\backup $\sim 5 \backslash$ valdemarlespec $\sim 1 \backslash$ spectro $\backslash$ vlj07b\noe $6 \backslash$ pdata $\backslash \backslash \backslash R$

Second Trace: $\backslash$ WINID \SPC\ASPX32\NOE\007001.1R

Aplicações de Técnicas de RMN À Determinação Estrutural de Intermediários Sintéticos 
irradiação na metila 8

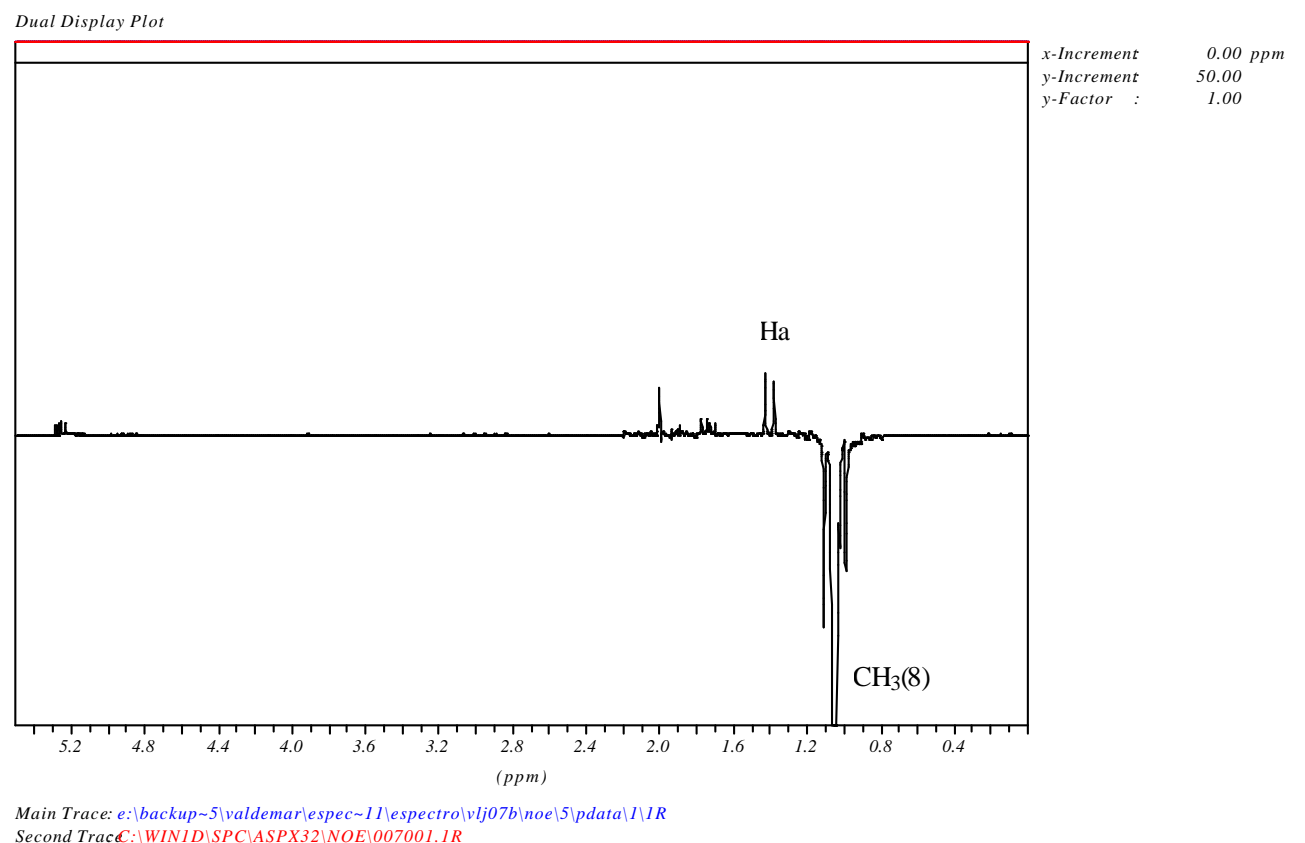

Tabela 26 - Dados espectrais de NOE DIFF do composto $7 \mathbf{b}\left(\mathrm{CDCl}_{3}\right)$.

\begin{tabular}{c|c|c}
$\delta$ (ppm)Irradiado & Atribuição & Efeito NOE Observado \\
& & \\
1,08 & $\mathrm{CH}_{3}(6)$ & $\mathrm{Ha}, \mathrm{Hc}$ \\
1,02 & $\mathrm{CH}_{3}(8)$ & $\mathrm{Ha}$ \\
1.01 & $\mathrm{CH}_{3}(7)$ & $\mathrm{Hb}, \mathrm{Hd}, \mathrm{He}$ \\
\hline
\end{tabular}

Tabela 27 - Dados espectrais de ${ }^{1} \mathrm{H}$ e ${ }^{13} \mathrm{C} \mathrm{RMN}$ para o $( \pm)$ - trans - 2-acetoxi 1,4,4 - trimetil-ciclopentano-1-carboxaldeído $\left(\mathrm{CDCl}_{3}\right)$

\begin{tabular}{|c|c|c|}
\hline $\mathrm{C}-, \mathrm{H}-$ & ${ }^{1} \mathrm{H}(300 \mathrm{MHz}) \delta(\mathrm{ppm})$ & $\begin{array}{c}{ }^{13} \mathrm{C} \\
(75 \mathrm{MHz})\end{array}$ \\
\hline 1 & $\mathrm{He}-5,31(\mathrm{dd}, 1 \mathrm{H}, \mathrm{Jec}=8,7$ e Jed=6,5 Hz $)$ & 76,39 \\
\hline 2 & $\begin{array}{c}\text { Hd-1,93 (ddd, } 1 \mathrm{H}, \mathrm{Jdc}=13 ; \mathrm{Jde}=6,5 \text { e Jdb=1,2 Hz) e Hc-1,78 } \\
(\mathrm{dd}, 1 \mathrm{H}, \mathrm{Jcd}=13 \text { e Jce }=8,7 \mathrm{~Hz})\end{array}$ & 44,94 \\
\hline 3 & & 35,15 \\
\hline 4 & $\begin{array}{c}\text { Hb-1,97 (dd, 1H, Jba=13,9 e Jbd=1,2 Hz) e Ha-1,45 (d, 1H, } \\
\text { Jab= 13,9Hz) }\end{array}$ & 47,93 \\
\hline 5 & & 56,45 \\
\hline 6 & $1,08(\mathrm{~s}, 3 \mathrm{H})$ & 31,18 \\
\hline 7 & $1,01(\mathrm{~s}, 3 \mathrm{H})$ & 30,33 \\
\hline 8 & $1,02(\mathrm{~s}, 3 \mathrm{H})$ & 15,9 \\
\hline 9 & $9,52(\mathrm{~s}, 1 \mathrm{H})$ & 200,42 \\
\hline 10 & & 170,37 \\
\hline 11 & $2,04(\mathrm{~s}, 3 \mathrm{H})$ & 20,58 \\
\hline
\end{tabular}




\section{Composto (8a)}

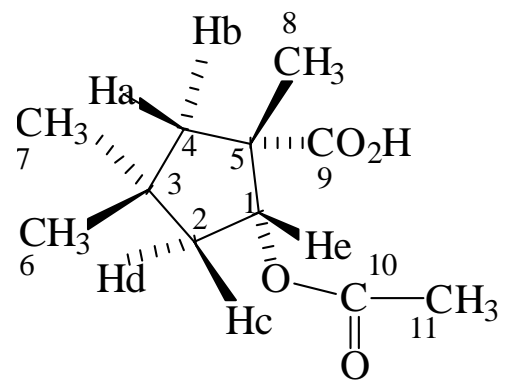

Espectro de ${ }^{1} \mathrm{H}$-RMN do composto (8a)

$8 \mathbf{a}$

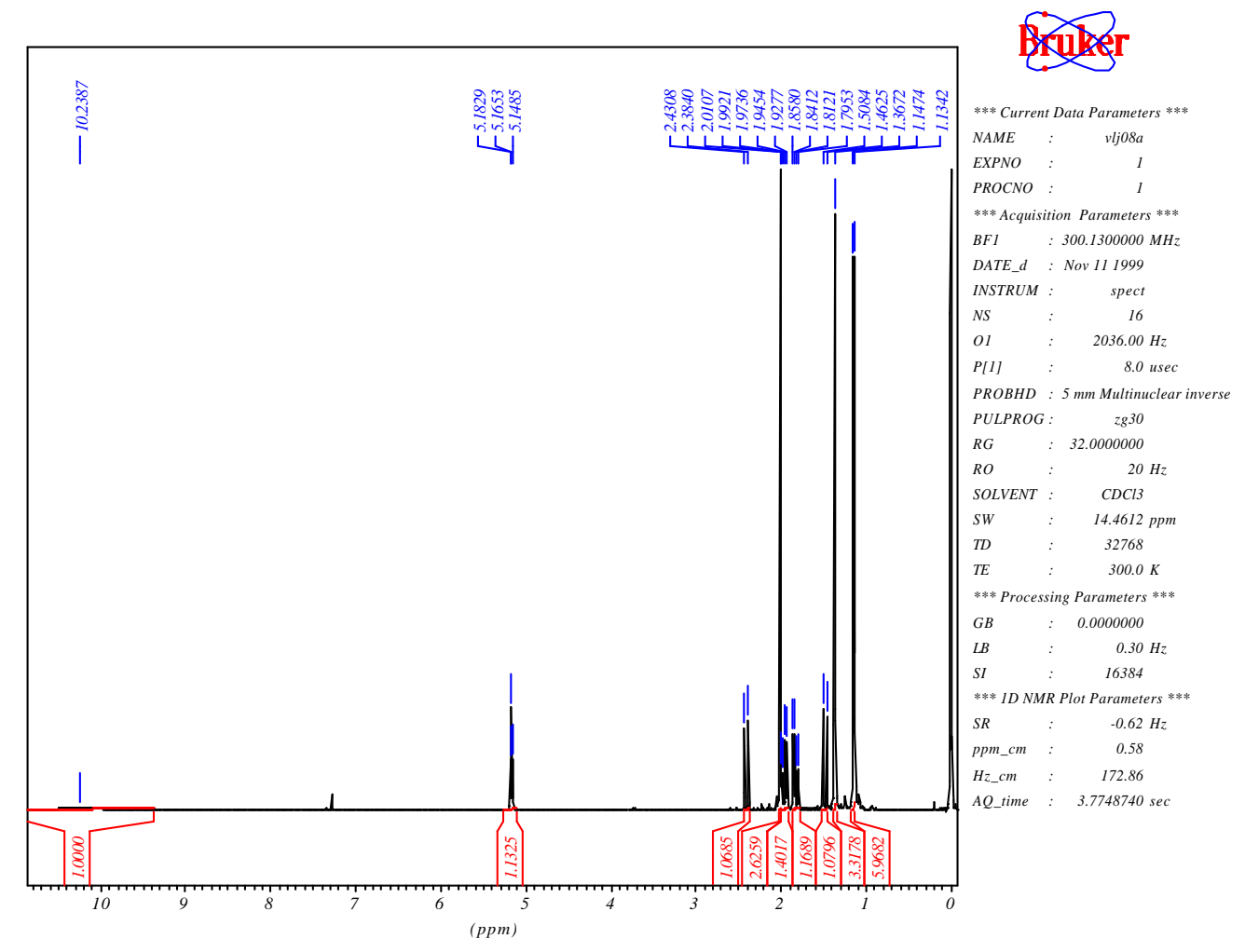

Tabela 28 - Dados espectrais de ${ }^{1} \mathrm{H}$ RMN do composto 8a $\left(\mathrm{CDCl}_{3}\right)$.

\begin{tabular}{l|l|c|c}
\hline$\delta(\mathbf{p p m})$ & Atribuição & sinal & J $(\mathbf{H z})$ \\
\hline 10,21 & $(9)$ & $\mathrm{sl}$ & \\
5,17 & $\mathrm{He}$ & $\mathrm{t}$ & $\mathrm{Jec}=\mathrm{Jed}=5,2 \mathrm{~Hz}$ \\
2,41 & $\mathrm{Hb}$ & $\mathrm{d}$ & $\mathrm{Jba}=14 \mathrm{~Hz}$ \\
2,04 & $\mathrm{CH}_{3}(11)$ & $\mathrm{s}$ & $\mathrm{Jcd}=13,8 ; \mathrm{Jce}=5,2$ e Jca $=0,6$ \\
1,96 & $\mathrm{Hc}$ & $\mathrm{ddd}$ & $\mathrm{Hz}$ \\
& & $\mathrm{dd}$ & $\mathrm{Jdc}=13,8 ; \mathrm{Jde}=5,2 \mathrm{~Hz}$ \\
1,83 & $\mathrm{Hd}$ & $\mathrm{dd}$ & $\mathrm{Jab}=14 \mathrm{e} \mathrm{Jac}=0,6 \mathrm{~Hz}$ \\
1,49 & $\mathrm{Ha}$ & $\mathrm{s}$ & \\
1,36 & $\mathrm{CH}_{3}(8)$ & $\mathrm{s}$ & \\
1,14 & $\mathrm{CH}_{3}(7)$ & $\mathrm{s}$ & \\
1,13 & $\mathrm{CH}_{3}(6)$ & & \\
\hline
\end{tabular}




\section{Espectro de ${ }^{13}$ C-RMN (BB) do composto (8a)}

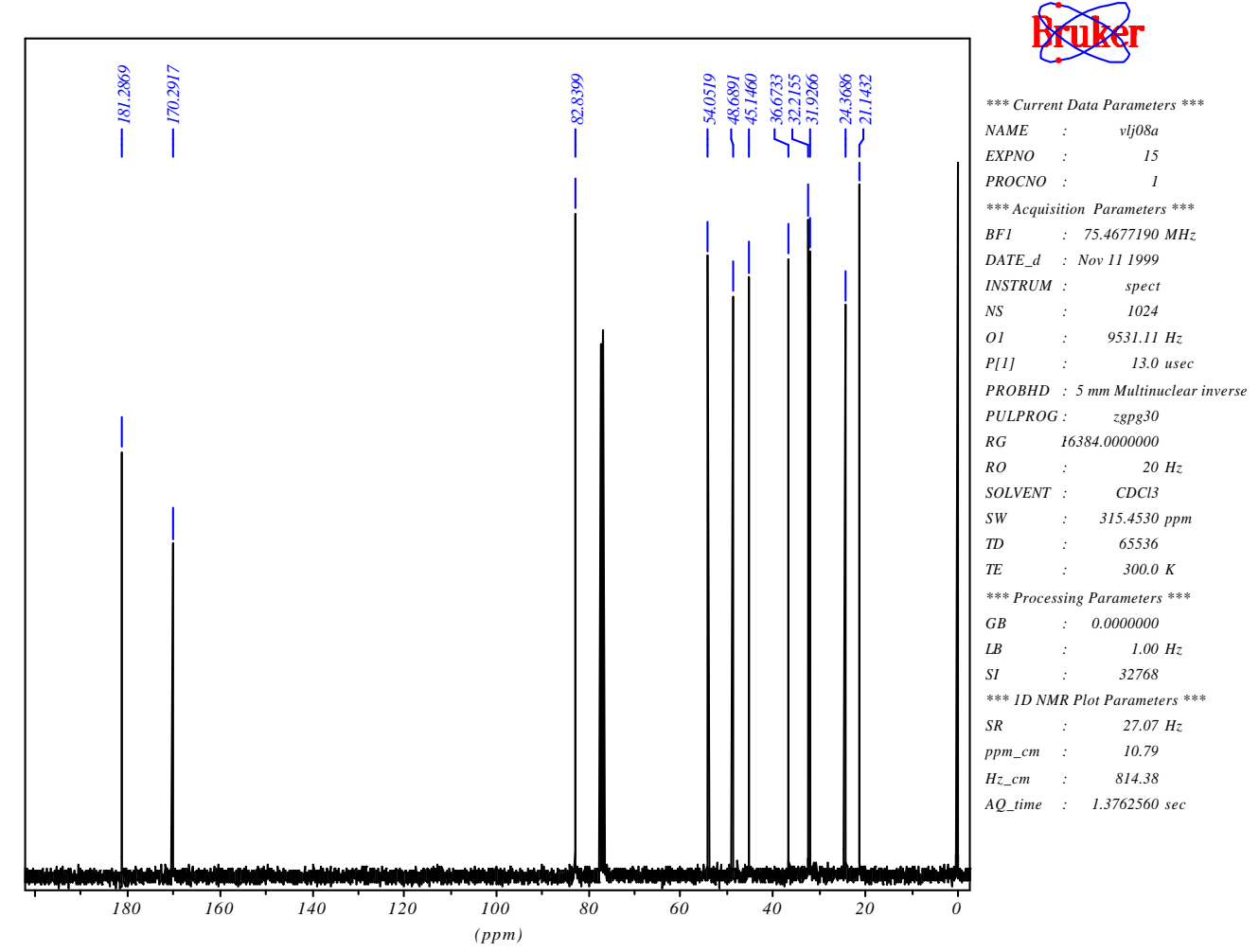

Espectro de ${ }^{13} \mathrm{C}$-RMN (DEPT-135) do composto (8a)

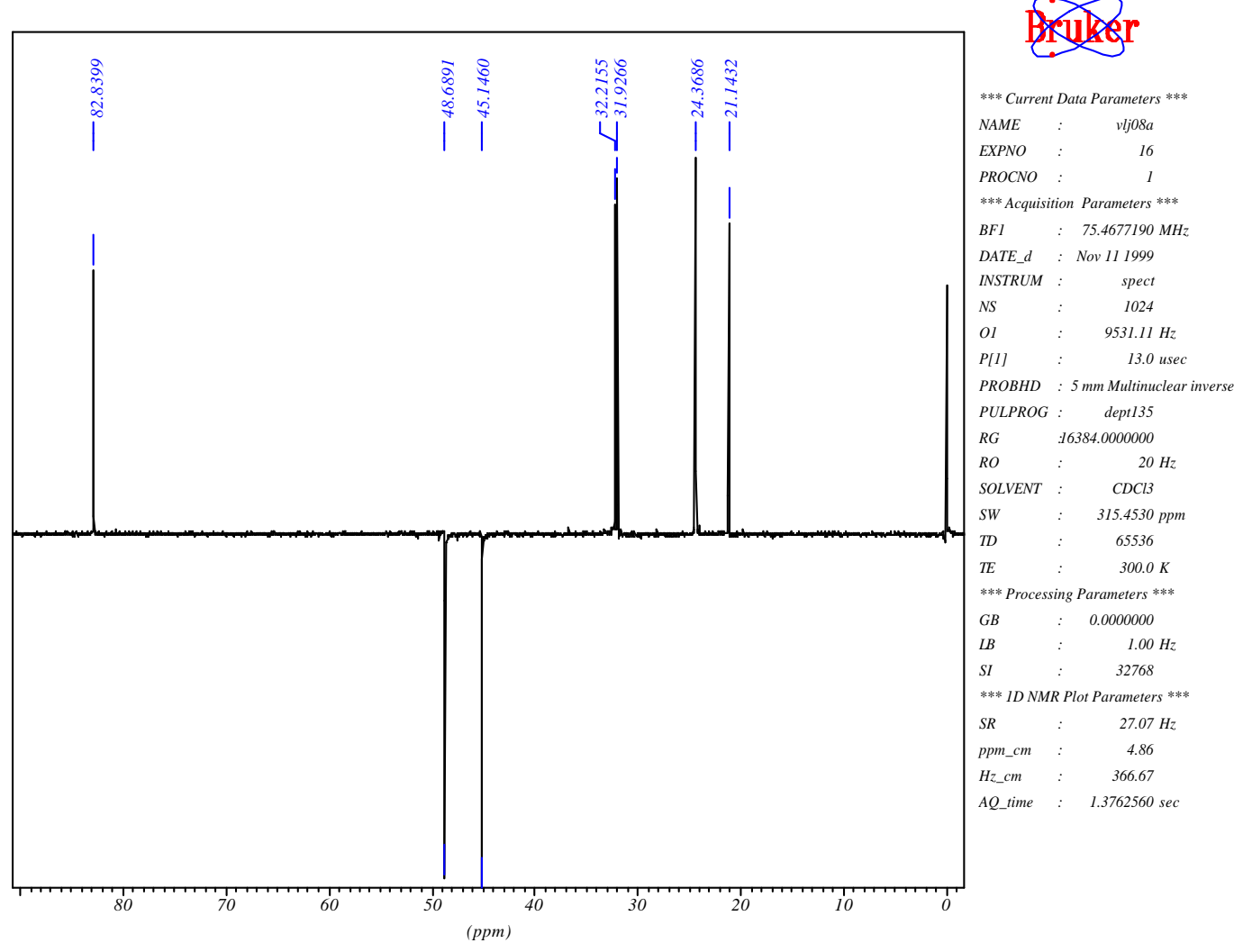




\section{Espectro HMQC (2D H/C) do composto (8a)}
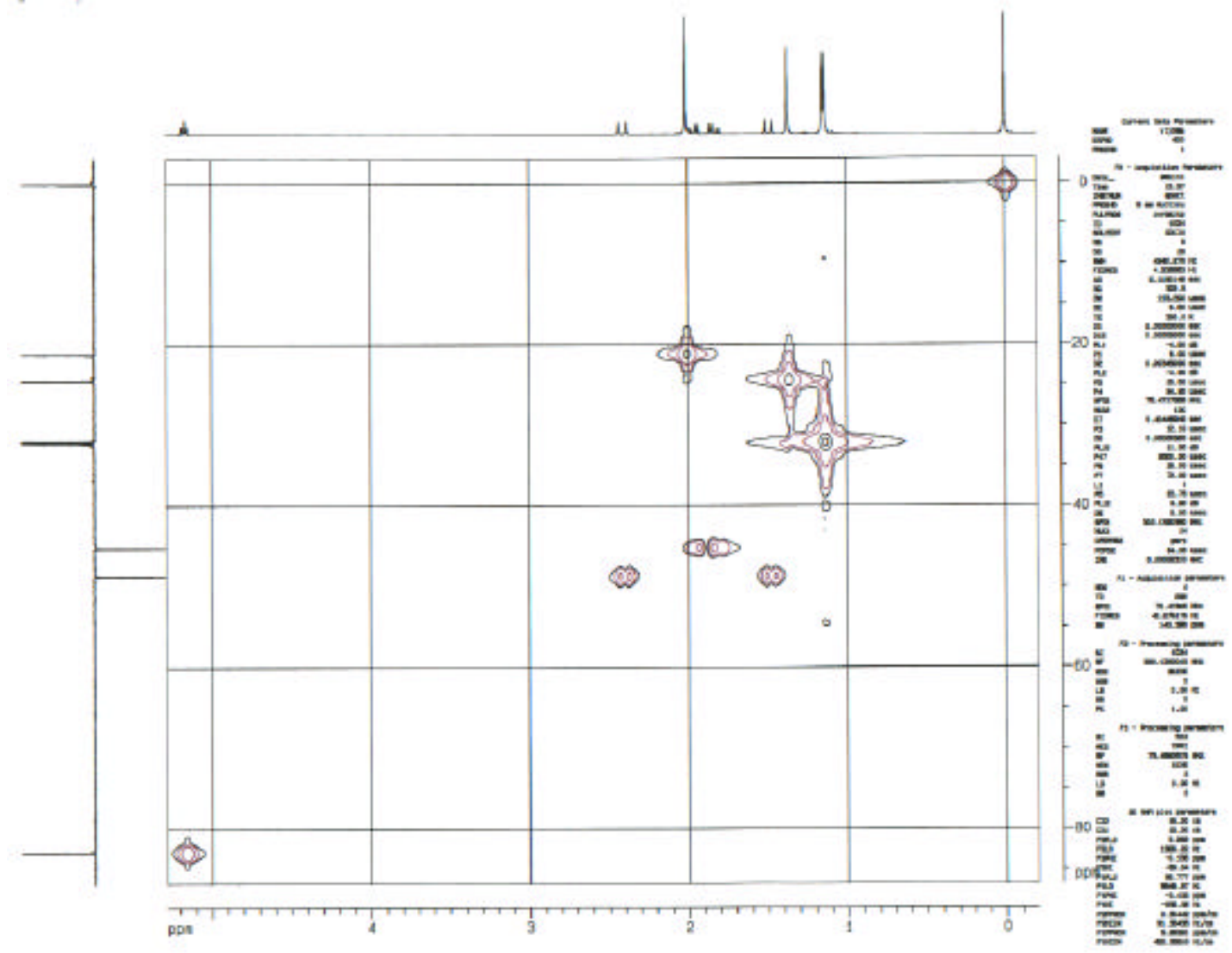

Espectro Jres do composto (8a)
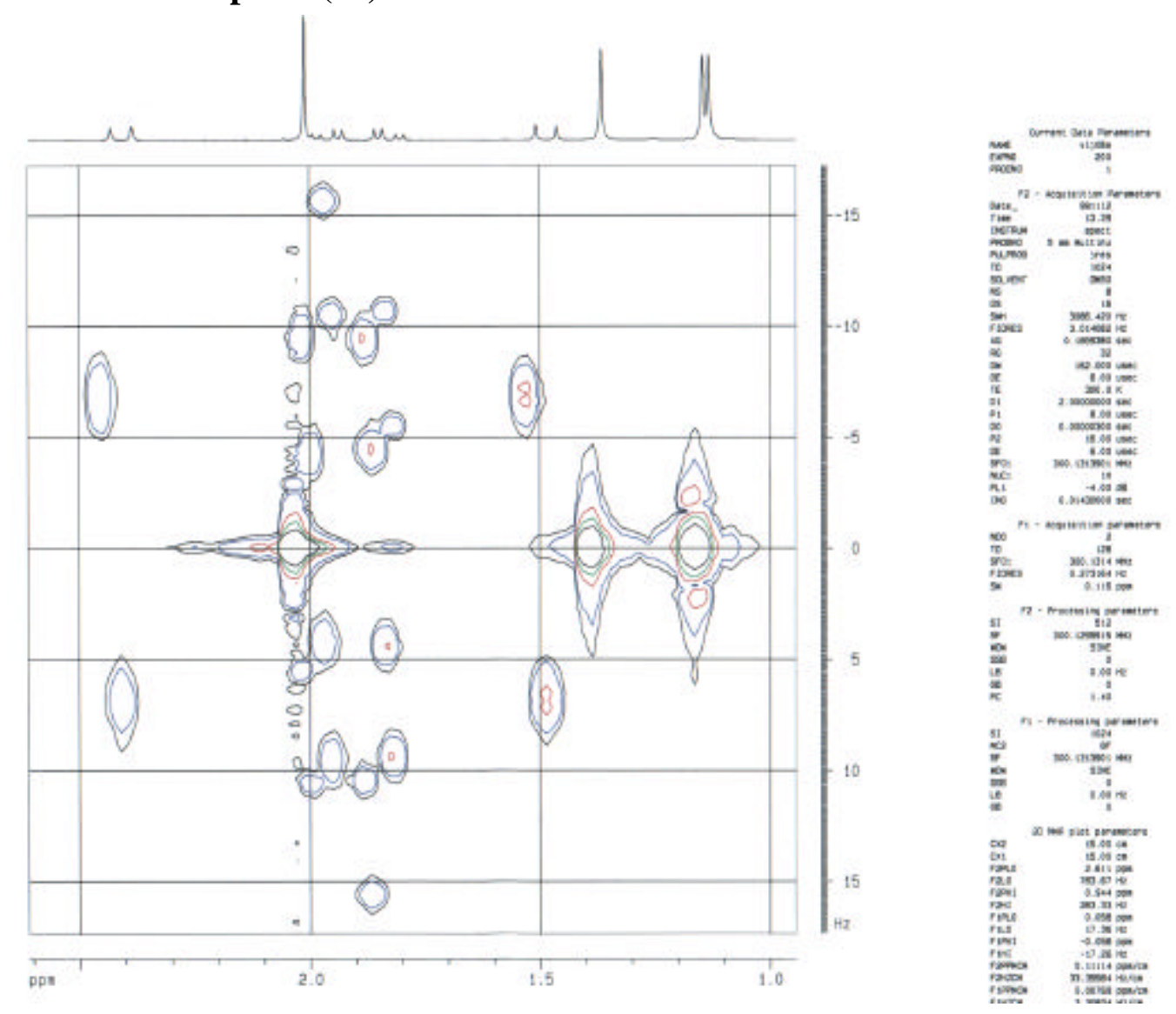


\section{Espectros NOE DIFF do composto (8a)}

$>$ irradiação na metila 6

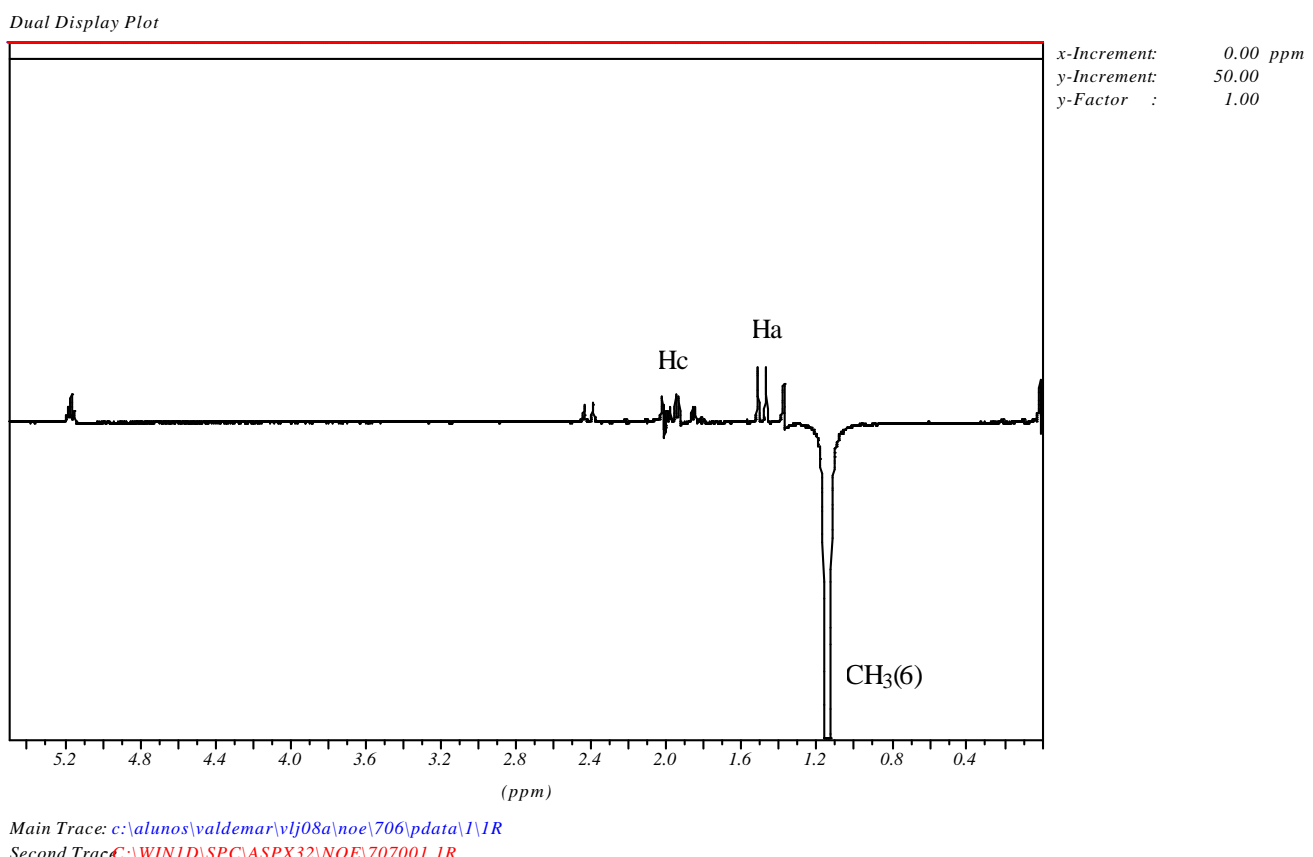

Second TracC: $I W I N I D T S P C A S P X 32 W O E T 707001$ IR

irradiação na metila 7

Dual Display Plot

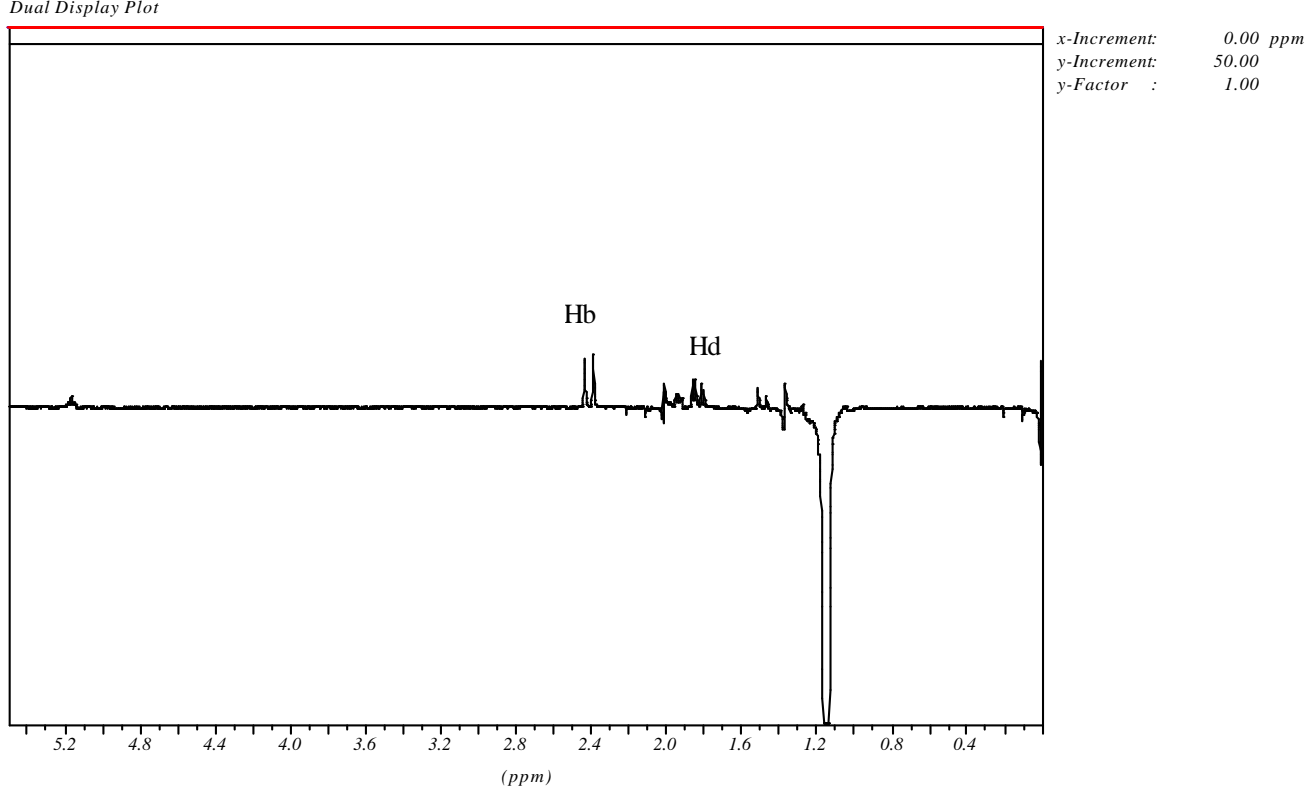

Main Trace: $c$ : \alunos\valdemar $\backslash v l j 08 a \backslash n o e \backslash 703 \backslash p d a t a \backslash \backslash \backslash \backslash R$

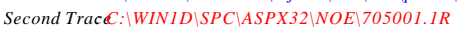


irradiação na metila 8

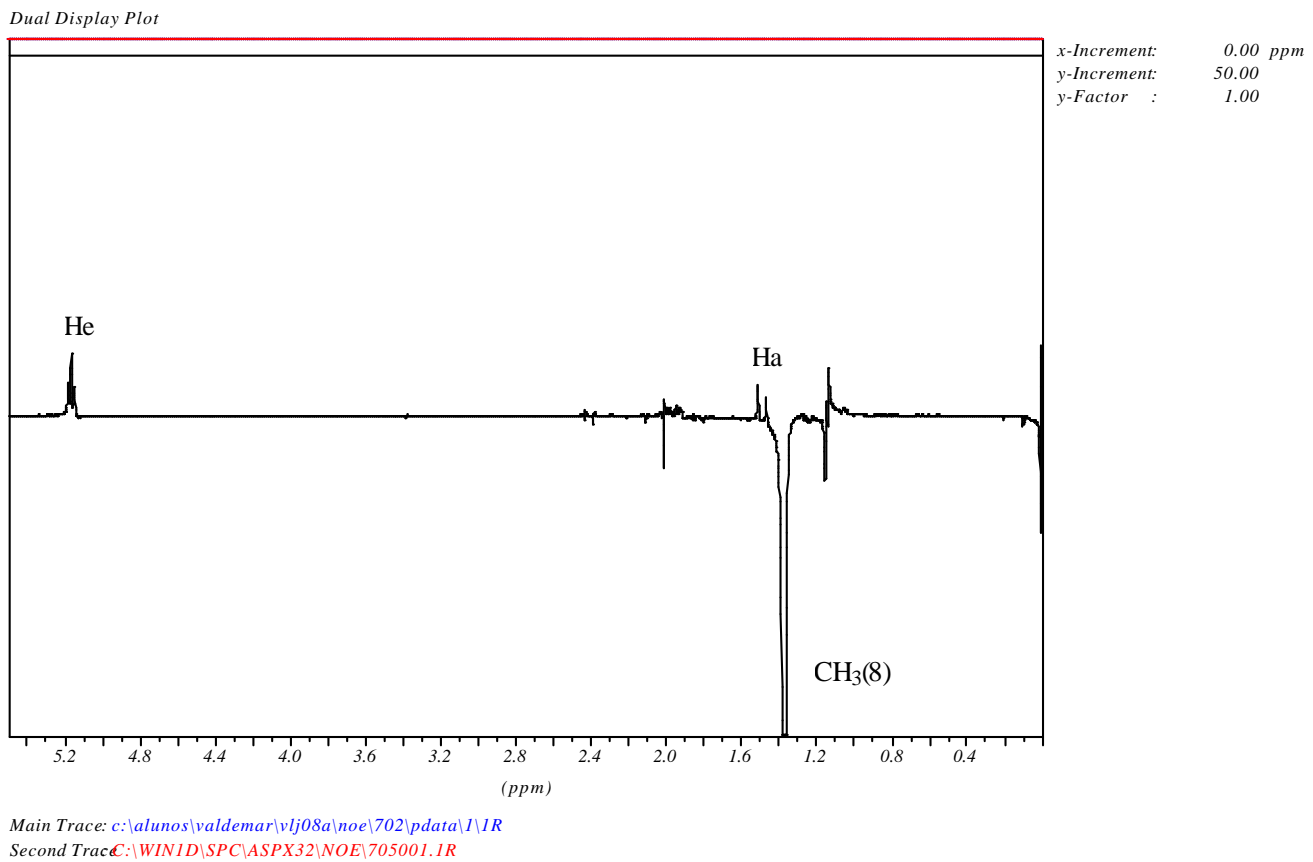

Tabela 29 - Dados espectrais de NOE DIFF do composto 8a $\left(\mathrm{CDCl}_{3}\right)$.

$\delta(\mathbf{p p m})$ Irradiado

1,36
1,14
1.13
Atribuição

$\mathrm{CH}_{3}(8)$

$\mathrm{CH}_{3}(7)$

$\mathrm{CH}_{3}(6)$

\section{Efeito NOE Observado}

$\mathrm{Ha}, \mathrm{He}$

$\mathrm{Hb}, \mathrm{Hd}$

$\mathrm{Ha}, \mathrm{Hc}$

Tabela 30 - Dados espectrais de ${ }^{1} \mathrm{H}$ e ${ }^{13} \mathrm{C} \mathrm{RMN}$ para o ( \pm ) -cis - 2-acetoxi - 1,4,4 trimetil-ciclopentano-1- ácido carboxílico $\left(\mathrm{CDCl}_{3}\right)$

\begin{tabular}{|c|c|c|}
\hline C-, H- & ${ }^{1} \mathrm{H}(300 \mathrm{MHz}) \delta(\mathrm{ppm})$ & ${ }^{13} \mathrm{C}(75 \mathrm{MHz})$ \\
\hline 1 & $\mathrm{He}-5,17(\mathrm{t}, 1 \mathrm{H}, \mathrm{Jec}=\mathrm{Jed}=5,2 \mathrm{~Hz})$ & 82,83 \\
\hline 2 & $\begin{array}{c}\text { Hc-1,96 (ddd, 1H, Jcd=13,8; Jce=5,2 e Jca }=0,6 \mathrm{~Hz}) \text { e Hd-1,83 } \\
(\mathrm{dd}, 1 \mathrm{H}, \mathrm{Jdc}=13,8 ; \mathrm{Jde}=5,2 \mathrm{~Hz})\end{array}$ & 45,14 \\
\hline 3 & & 36,67 \\
\hline 4 & $\begin{array}{l}\mathrm{Hb}-2,41(\mathrm{~d}, 1 \mathrm{H}, \mathrm{Jba}=14 \mathrm{~Hz}) \text { e Ha-1,49 }(\mathrm{dd}, 1 \mathrm{H}, \mathrm{Jab}=14 \mathrm{e} \\
\mathrm{Jac}=0,6 \mathrm{~Hz})\end{array}$ & 48,68 \\
\hline 5 & & 54,05 \\
\hline 6 & $1,13(\mathrm{~s}, 3 \mathrm{H})$ & $32,21 *$ \\
\hline 7 & $1,14(\mathrm{~s}, 3 \mathrm{H})$ & $31,92 *$ \\
\hline 8 & $1,36(\mathrm{~s}, 3 \mathrm{H})$ & 24,36 \\
\hline 9 & $10,21(\mathrm{sl}, 1 \mathrm{H}, \mathrm{OH})$ & 181,28 \\
\hline 10 & & 170,29 \\
\hline 11 & $2,01(\mathrm{~s}, 3 \mathrm{H})$ & 21,14 \\
\hline
\end{tabular}

*Podem estar invertidos 


\section{Composto (8b)}

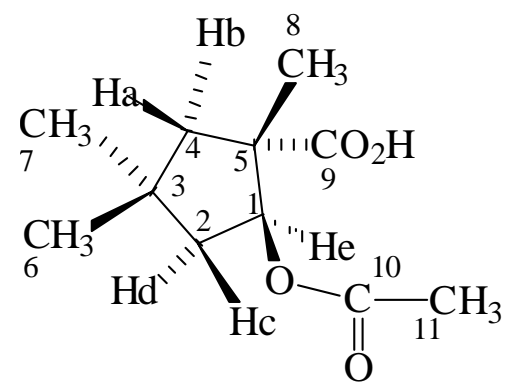

\section{Espectro de ${ }^{1} \mathrm{H}$-RMN do composto $(8 \mathrm{~b})$}

\section{$8 b$}

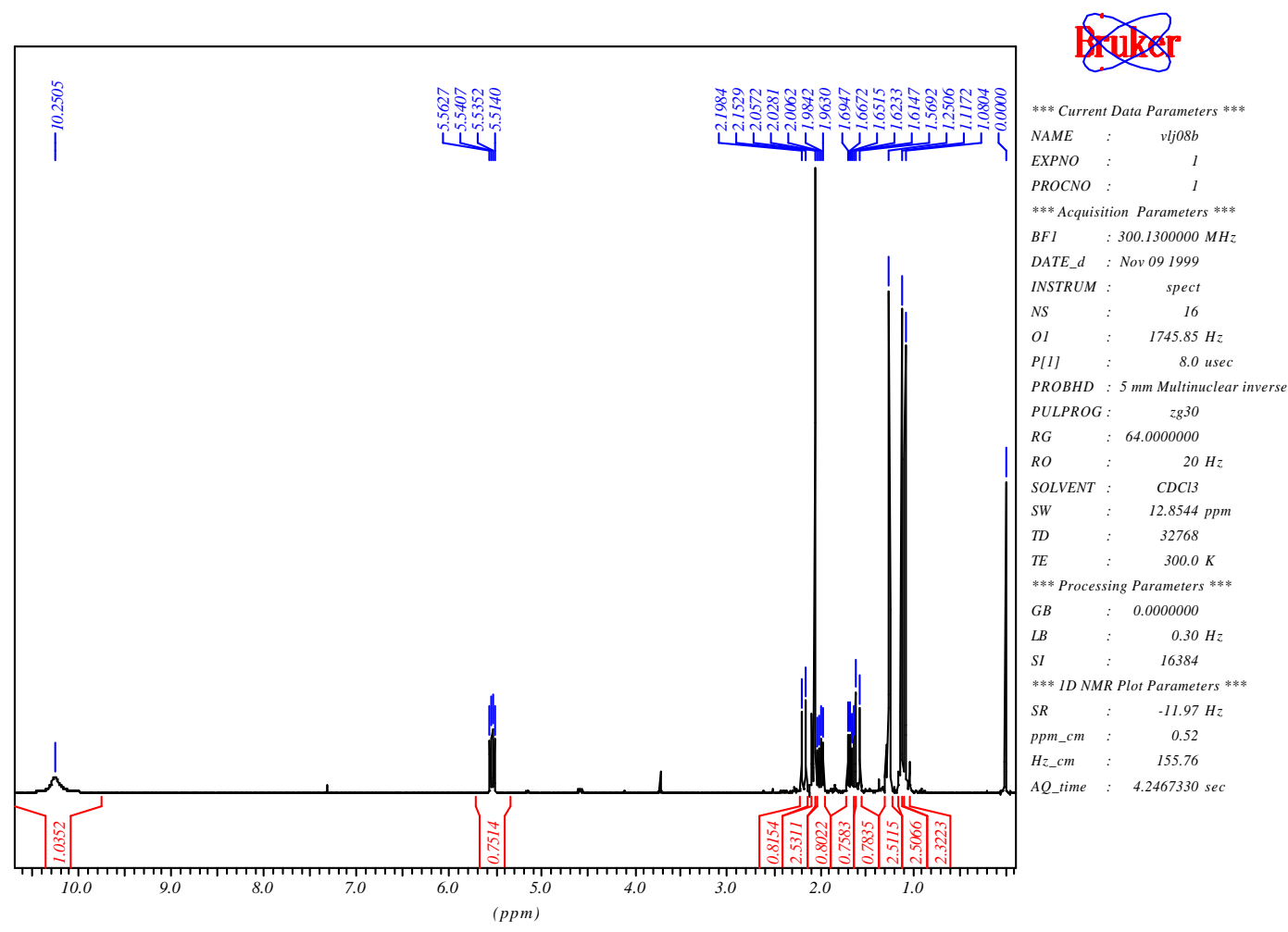

Tabela 31 - Dados espectrais de ${ }^{1} \mathrm{H}$ RMN do composto $\mathbf{8 b}\left(\mathrm{CDCl}_{3}\right)$.

\begin{tabular}{l|l|l|l}
\hline$\delta(\mathbf{p p m})$ & Atribuição & sinal & J (Hz) \\
\hline 10,25 & $(9)$ & $\mathrm{sl}$ & \\
5,54 & $\mathrm{He}$ & $\mathrm{dd}$ & $\mathrm{Jec}=8,1 \mathrm{e} \mathrm{Jed}=6,6 \mathrm{~Hz}$ \\
2,17 & $\mathrm{Hb}$ & $\mathrm{dd}$ & $\mathrm{Jba}=13,6 \mathrm{e} \mathrm{Jbd}=2,6 \mathrm{~Hz}$ \\
2,05 & $\mathrm{CH}_{3}(11)$ & $\mathrm{s}$ & \\
1,99 & $\mathrm{Hd}$ & $\mathrm{ddd}$ & $\mathrm{Jdc}=13,1 ; \mathrm{Jde}=6,6 \mathrm{e}$ \\
& & & $\mathrm{Jdb}=2,6 \mathrm{~Hz}$ \\
1,66 & $\mathrm{Hc}$ & $\mathrm{dd}$ & $\mathrm{Jcd}=13,1 \mathrm{e} \mathrm{Jce}=8,1 \mathrm{~Hz}$ \\
1,59 & $\mathrm{Ha}$ & $\mathrm{dd}$ & $\mathrm{Jab}=13,6 \mathrm{~Hz}$ \\
1,25 & $\mathrm{CH}_{3}(8)$ & $\mathrm{s}$ & \\
1,11 & $\mathrm{CH}_{3}(6)$ & $\mathrm{s}$ & \\
1,08 & $\mathrm{CH}_{3}(7)$ & $\mathrm{s}$ & \\
\hline
\end{tabular}




\section{Espectro de ${ }^{13}$ C-RMN (BB) do composto (8b)}

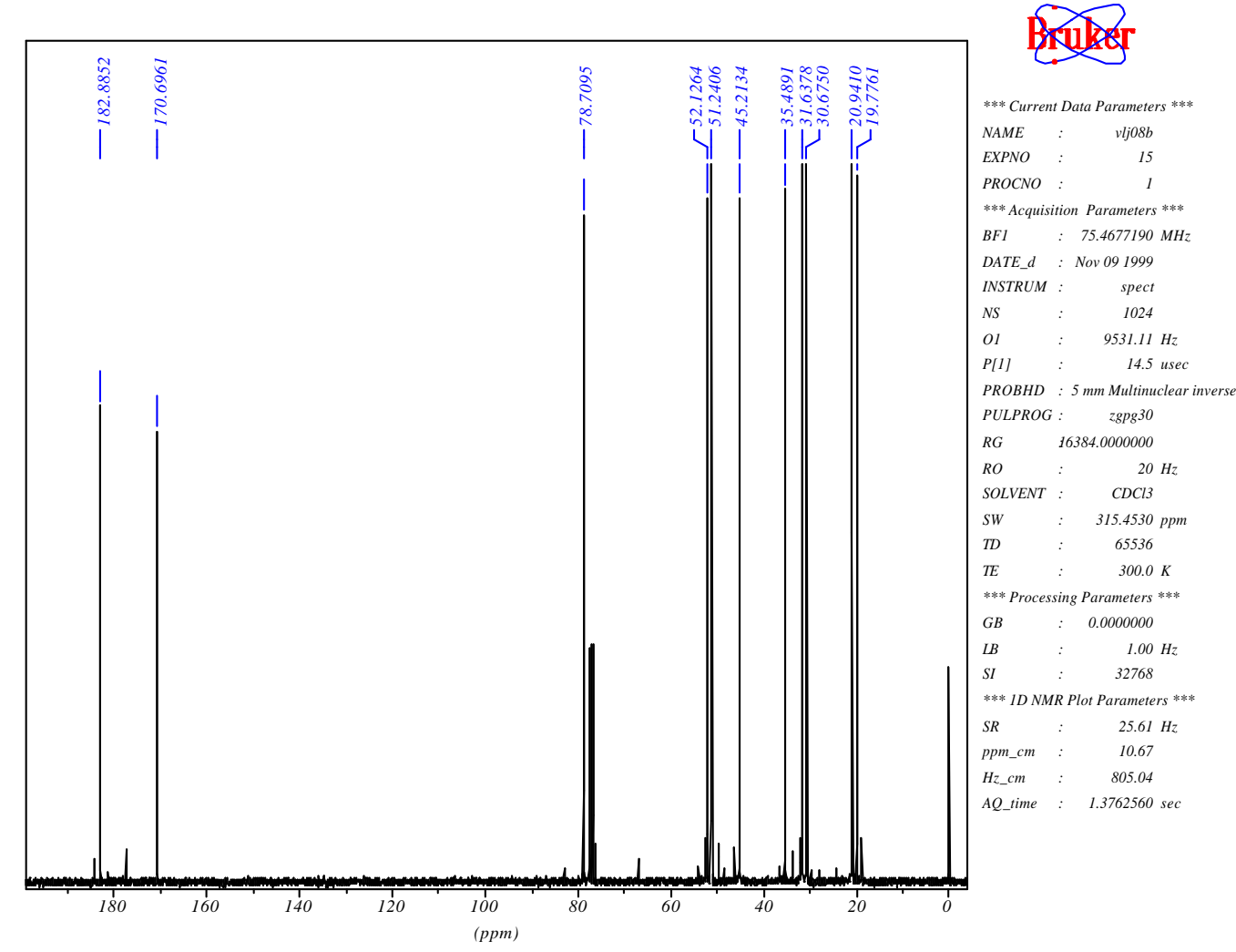

Espectro de ${ }^{13}$ C-RMN (DEPT-135) do composto (8b)

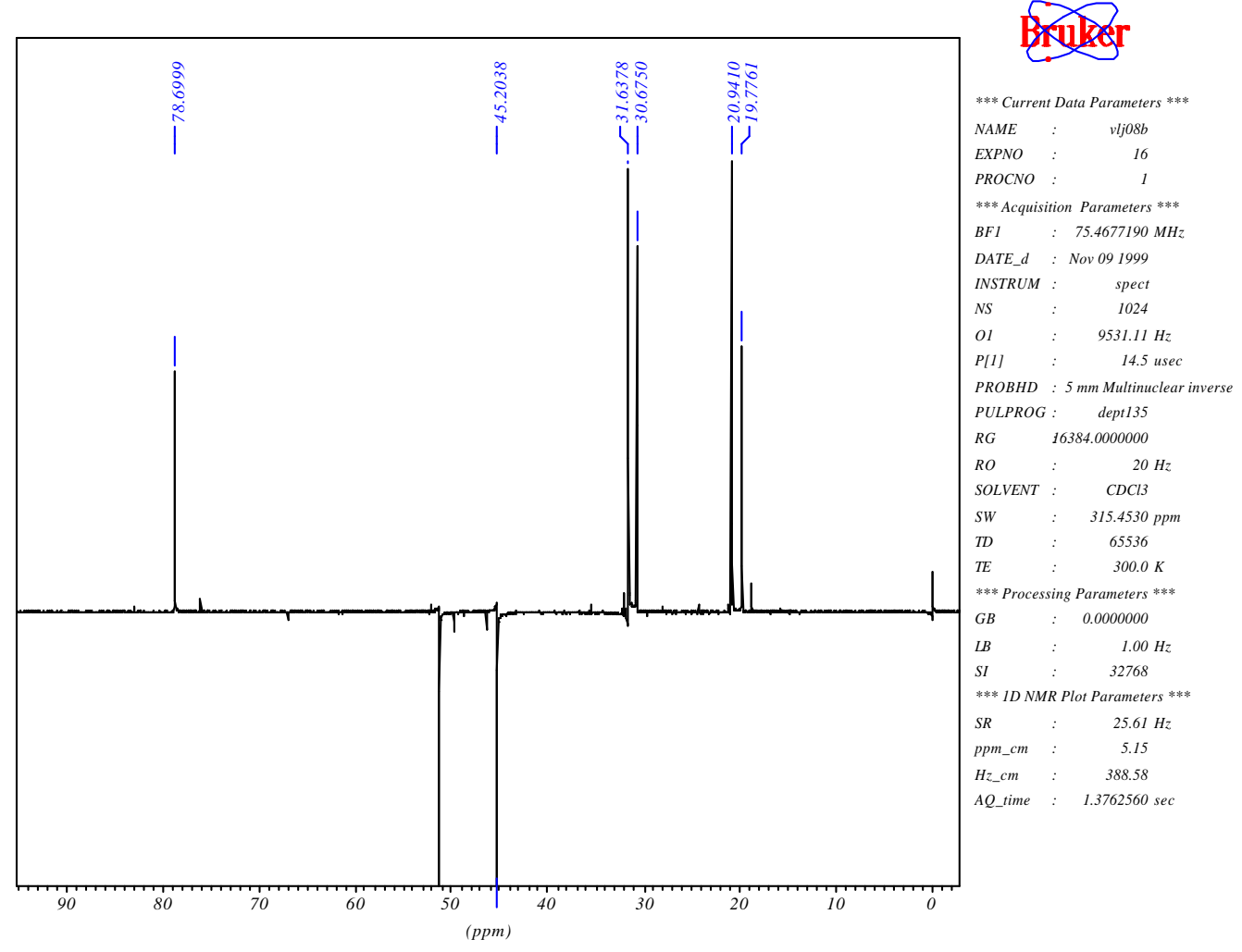


Espectro HMQC (2D H/C) do composto (8b)

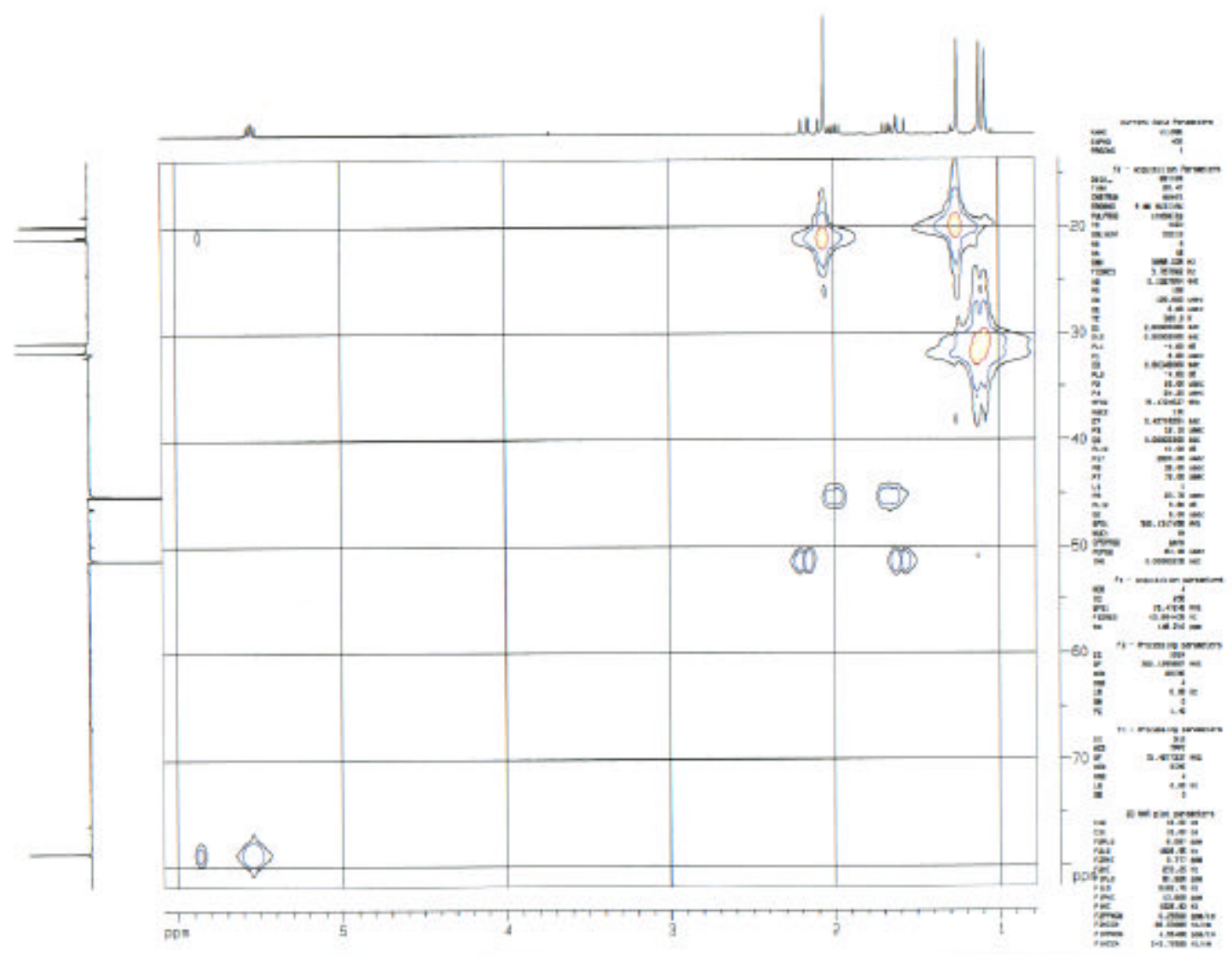

Espectro Jres do composto (8b)
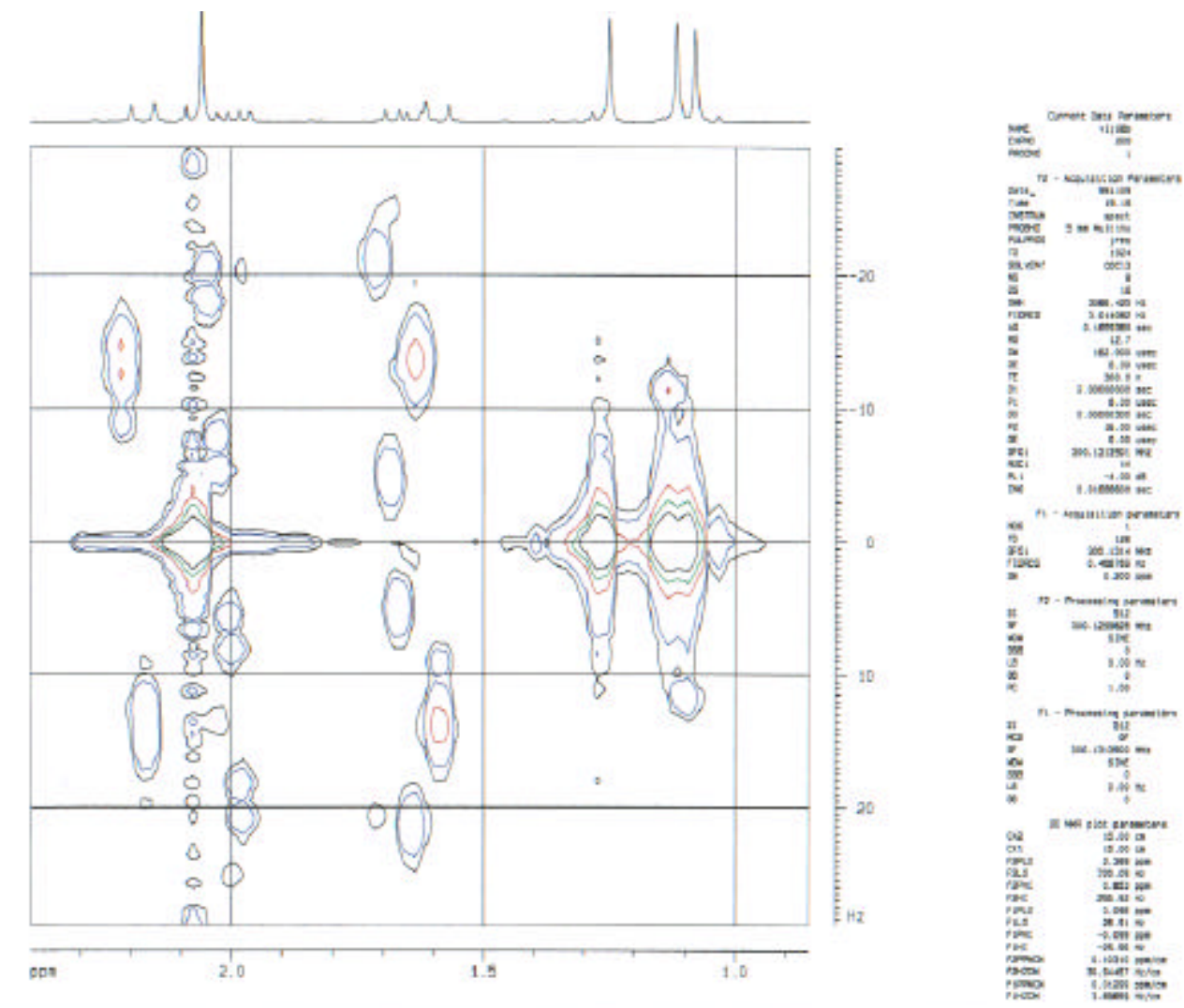


\section{Espectros NOE DIFF do composto (8b)}

$>$ irradiação na metila 6

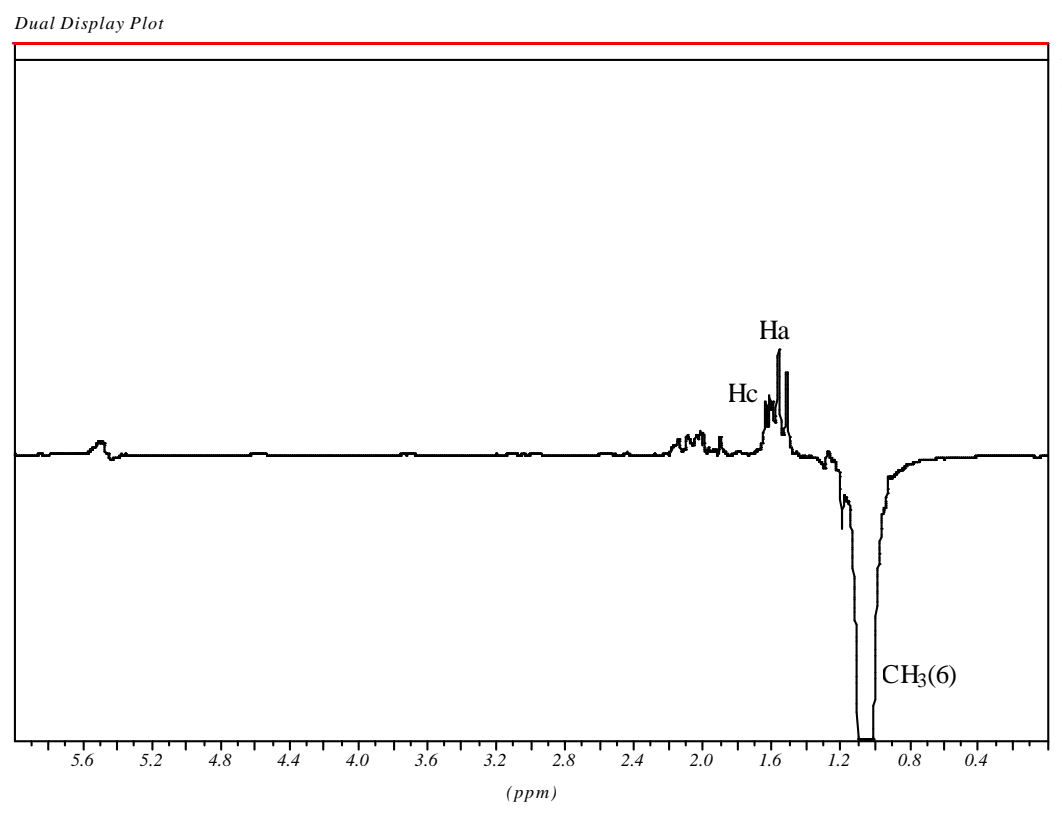

Main Trace: c:\alunos\valdemar $702 \backslash p d a t a \backslash \backslash \backslash R$

Second TracC: $\backslash W I N 1 D \backslash S P C \backslash A S P X 32 \backslash V A L D E M A R \backslash 704001.1 R$

$x$-Increment: $\quad 0.00 \mathrm{ppm}$

y-Increment: $\quad 50.00$

irradiação na metila 7

Dual Display Plot

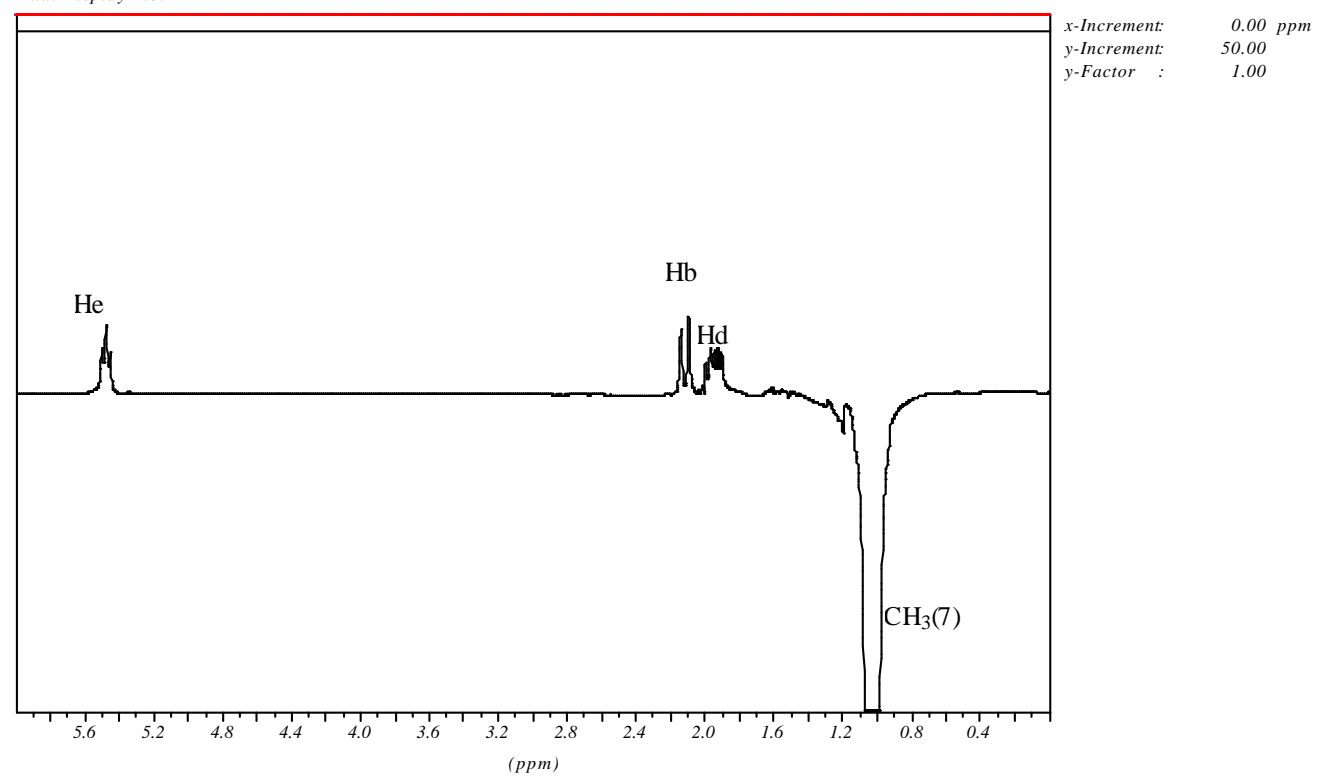

Main Trace: c: \alunos\valdemar $\backslash v l j 08 b \backslash$ noe $\backslash 703 \backslash$ data $\backslash \backslash \backslash I R$

Second Trace: $\backslash$ WINID SSPC\ASPX32\NOE\704001.1R 
irradiação na metila 8

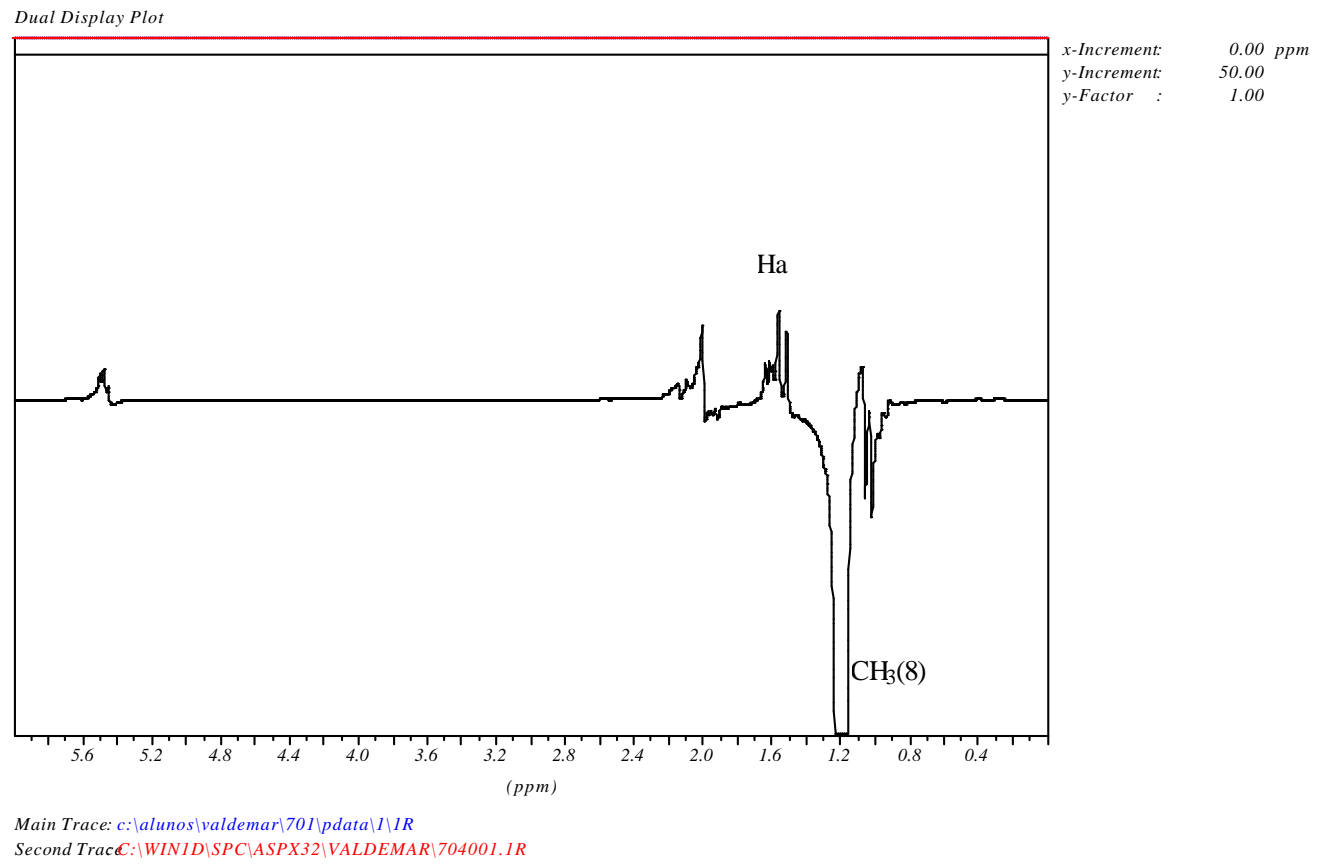

Tabela 32 - Dados espectrais de NOE DIFF do composto $\mathbf{8 b}\left(\mathrm{CDCl}_{3}\right)$.

\begin{tabular}{c|c|c}
\hline$\delta$ (ppm)Irradiado & Atribuição & Efeito NOE Observado \\
& & \\
1,25 & $\mathrm{CH}_{3}(8)$ & $\mathrm{Ha}$ \\
1,11 & $\mathrm{CH}_{3}(6)$ & $\mathrm{Ha}, \mathrm{Hc}$ \\
1,08 & $\mathrm{CH}_{3}(7)$ & $\mathrm{Hb}, \mathrm{Hd}, \mathrm{He}$ \\
\hline
\end{tabular}

Tabela 33 - Dados espectrais de ${ }^{1} \mathrm{H}$ e ${ }^{13} \mathrm{C}$ RMN para o ( \pm ) -trans - 2-acetoxi - 1,4,4 trimetil-ciclopentano-1- ácido carboxílico $\left(\mathrm{CDCl}_{3}\right)$

\begin{tabular}{|c|c|c|}
\hline $\mathrm{C}-_{-}, \mathrm{H}-$ & ${ }^{1} \mathrm{H}(300 \mathrm{MHz}) \delta(\mathrm{ppm})$ & ${ }^{13} \mathrm{C}(75 \mathrm{MHz})$ \\
\hline 1 & $\mathrm{He}-5,54(\mathrm{dd}, 1 \mathrm{H}, \mathrm{Jec}=8,1$ e Jed $=6,6 \mathrm{~Hz})$ & 78,69 \\
\hline 2 & $\begin{array}{c}\text { Hd-1,99 (ddd, } 1 \mathrm{H}, \mathrm{Jdc}=13,1 ; \mathrm{Jde}=6,6 \text { e Jdb=2,6 Hz) e Hc- } \\
1,66(\mathrm{dd}, 1 \mathrm{H}, \mathrm{Jcd}=13,1 \text { e Jce }=8,1 \mathrm{~Hz})\end{array}$ & 45,20 \\
\hline 3 & & 35,48 \\
\hline 4 & $\begin{array}{c}\mathrm{Hb}-2,17(\mathrm{dd}, 1 \mathrm{H}, \mathrm{Jba}=13,6 \text { e Jbd=2,6 Hz) e Ha-1,59 (d, 1H, } \\
\text { Jab= 13,6Hz) }\end{array}$ & 51,24 \\
\hline 5 & & 52,12 \\
\hline 6 & $1,11(\mathrm{~s}, 3 \mathrm{H})$ & $31,63^{*}$ \\
\hline 7 & $1,08(\mathrm{~s}, 3 \mathrm{H})$ & $30,67 *$ \\
\hline 8 & $1,25(\mathrm{~s}, 3 \mathrm{H})$ & 19,77 \\
\hline 9 & $10,25(\mathrm{sl}, 1 \mathrm{H}, \mathrm{OH})$ & 182,88 \\
\hline 10 & & 170,69 \\
\hline 11 & $2,05(\mathrm{~s}, 3 \mathrm{H})$ & 20,94 \\
\hline
\end{tabular}

*Podem estar invertidos 


\section{Composto (9a)}

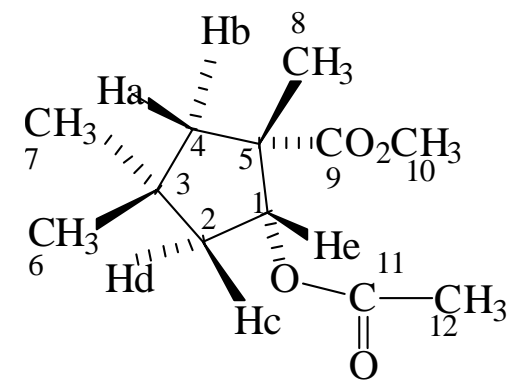

Espectro de ${ }^{1} \mathrm{H}-\mathrm{RMN}$ do composto (9a)

$9 a$

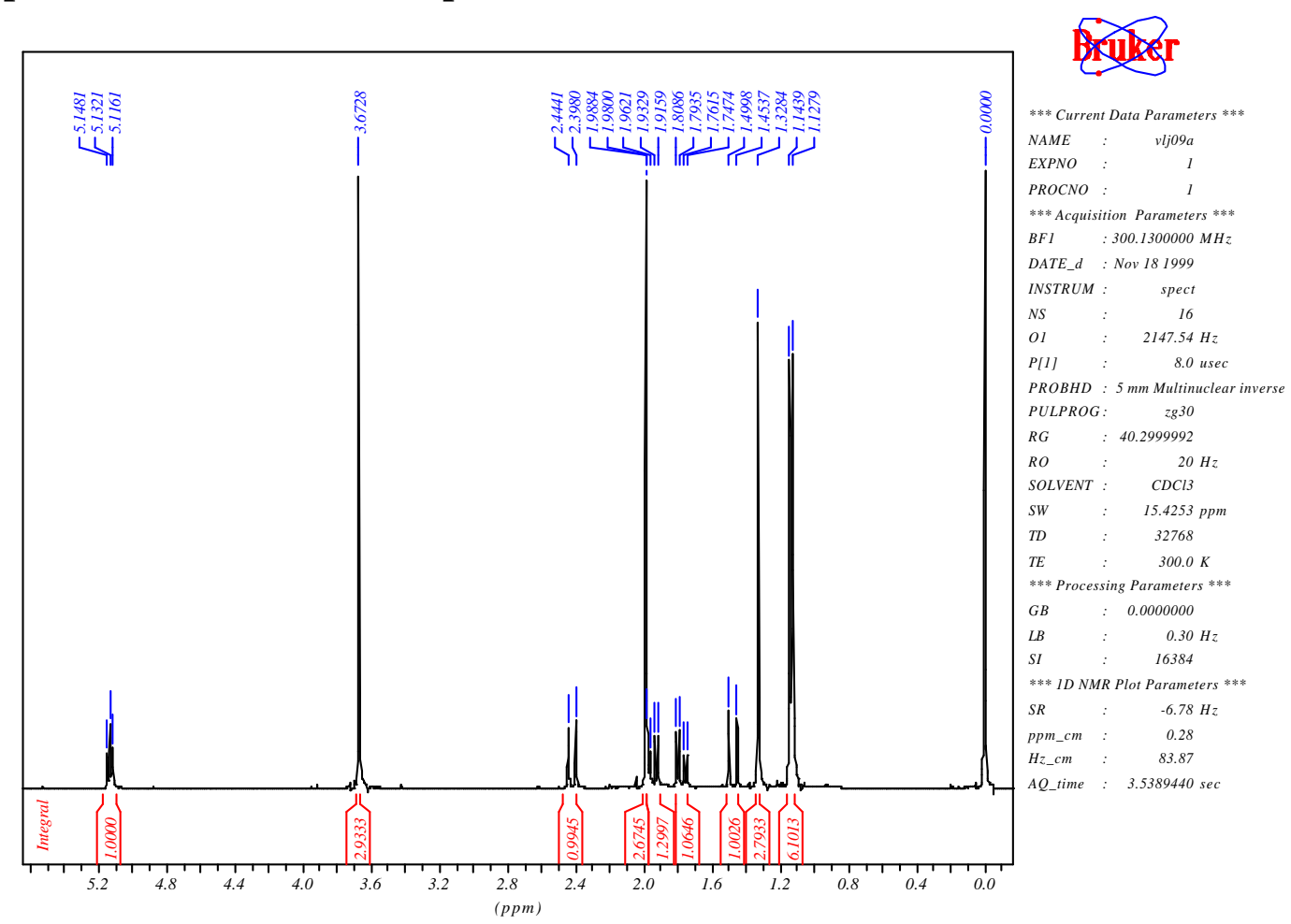

Tabela 34 - Dados espectrais de ${ }^{1} \mathrm{H}$ RMN do composto 9a $\left(\mathrm{CDCl}_{3}\right)$.

\begin{tabular}{c|l|c|c}
\hline$\delta(\mathbf{p p m})$ & Atribuição & sinal & J $(\mathbf{H z})$ \\
\hline 5,13 & $\mathrm{He}$ & $\mathrm{t}$ & $\mathrm{Jec}=\mathrm{Jed}=5 \mathrm{~Hz}$ \\
3,67 & $\mathrm{OCH}_{3}(10)$ & $\mathrm{s}$ & $\mathrm{Jba}=13,8 \mathrm{~Hz}$ \\
2,42 & $\mathrm{Hb}$ & $\mathrm{d}$ & \\
1,98 & $\mathrm{CH}_{3}(12)$ & $\mathrm{s}$ & $\mathrm{ddd}$ \\
1,95 & $\mathrm{Hc}$ & & $\mathrm{Jcd}=14 ; \mathrm{Jce}=5$ e Jca $=0,7$ \\
& & $\mathrm{dd}$ & $\mathrm{Hzd}=14 \mathrm{e} \mathrm{Jde}=5 \mathrm{~Hz}$ \\
1,78 & $\mathrm{Hd}$ & $\mathrm{dd}$ & $\mathrm{Jab}=13,8$ e Jac $=0,7 \mathrm{~Hz}$ \\
1,47 & $\mathrm{Ha}$ & $\mathrm{s}$ & \\
1,32 & $\mathrm{CH}_{3}(8)$ & $\mathrm{s}$ & \\
1,14 & $\mathrm{CH}_{3}(7)$ & $\mathrm{s}$ & \\
1,12 & $\mathrm{CH}_{3}(6)$ & & \\
\hline
\end{tabular}




\section{Espectro de ${ }^{13} \mathrm{C}-\mathrm{RMN}$ (BB) do composto (9a)}

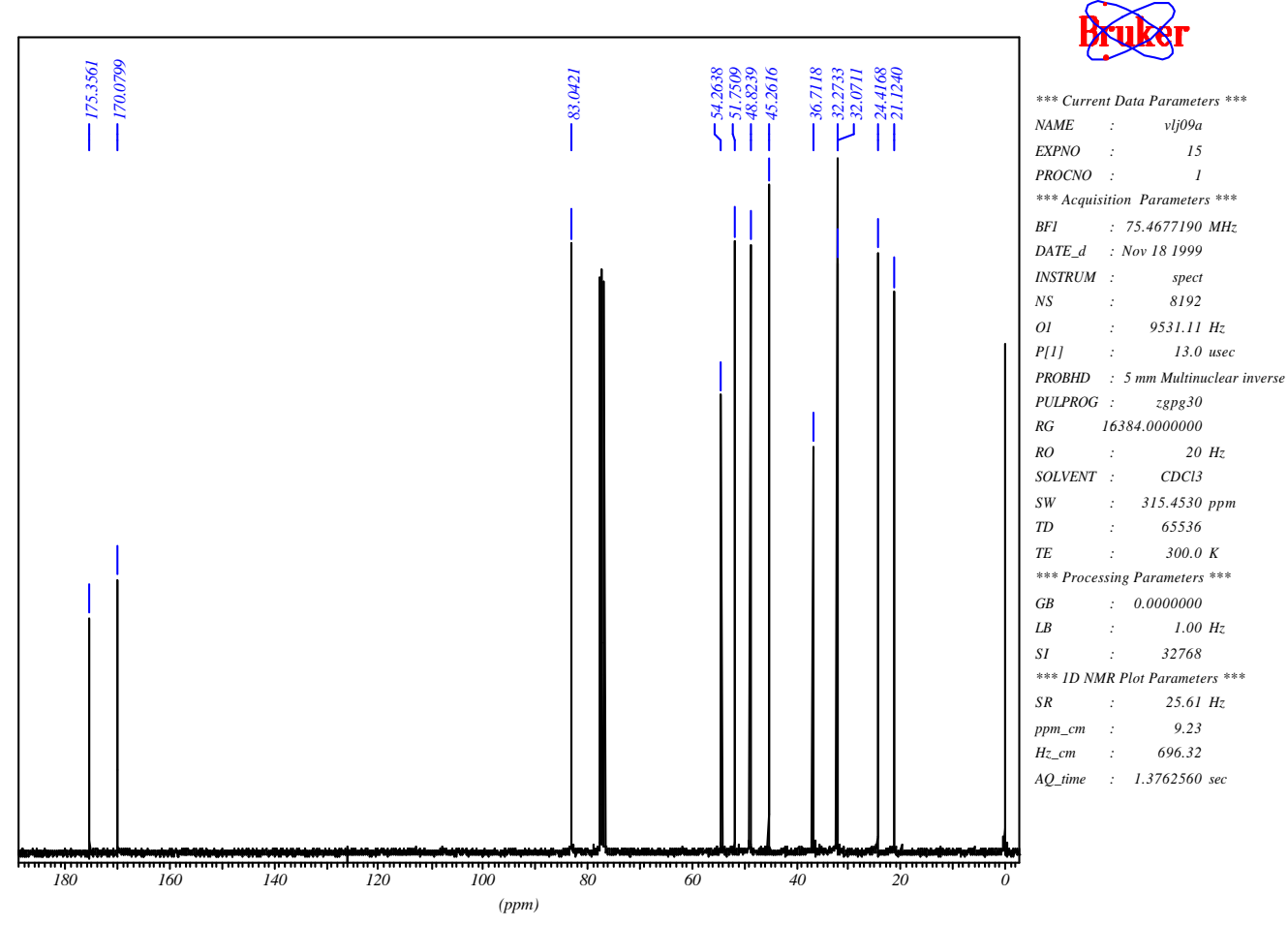

Espectro de ${ }^{13}$ C-RMN (DEPT-135) do composto (9a)

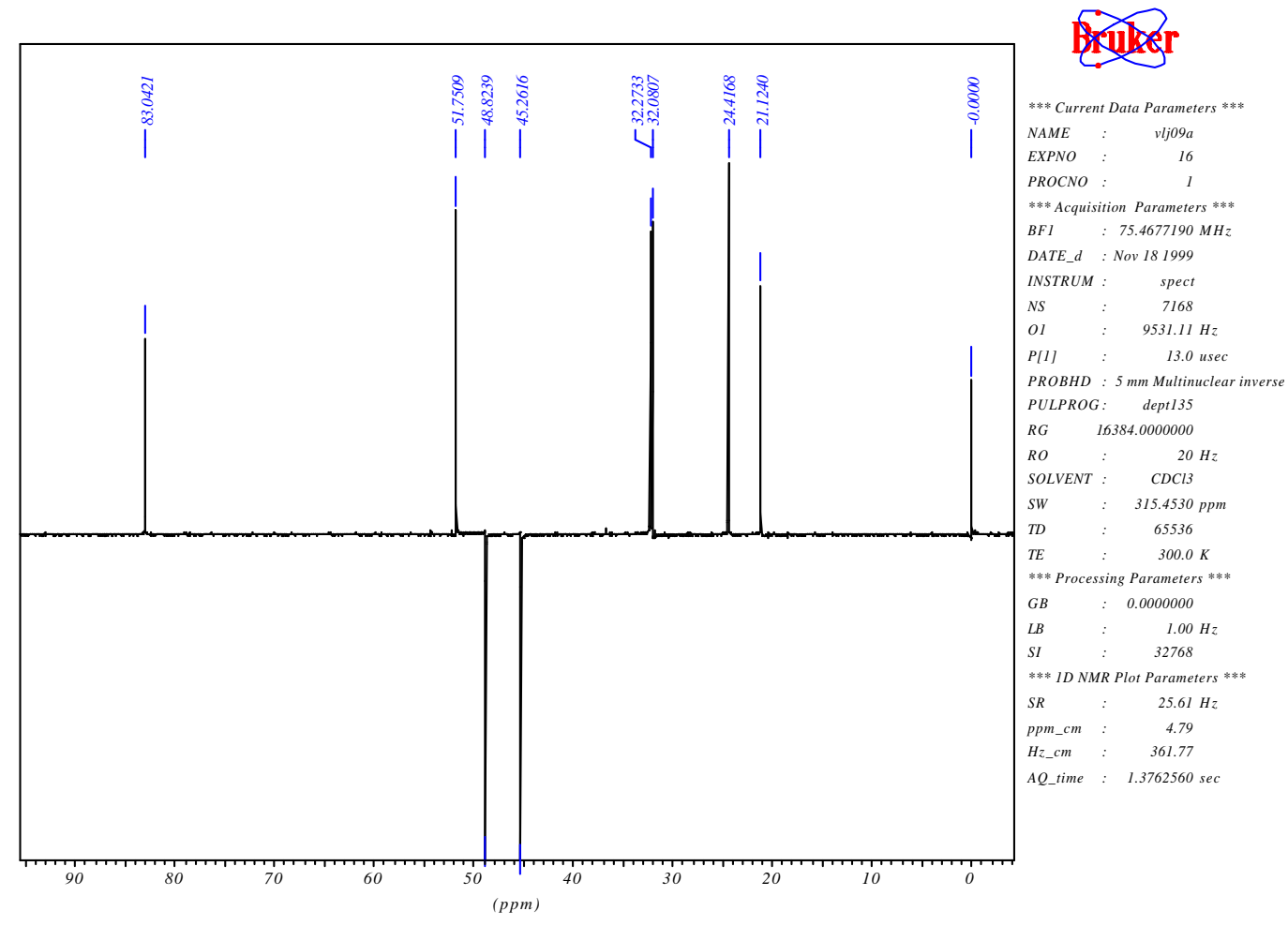




\section{Espectro HMQC (2D H/C) do composto (9a)}
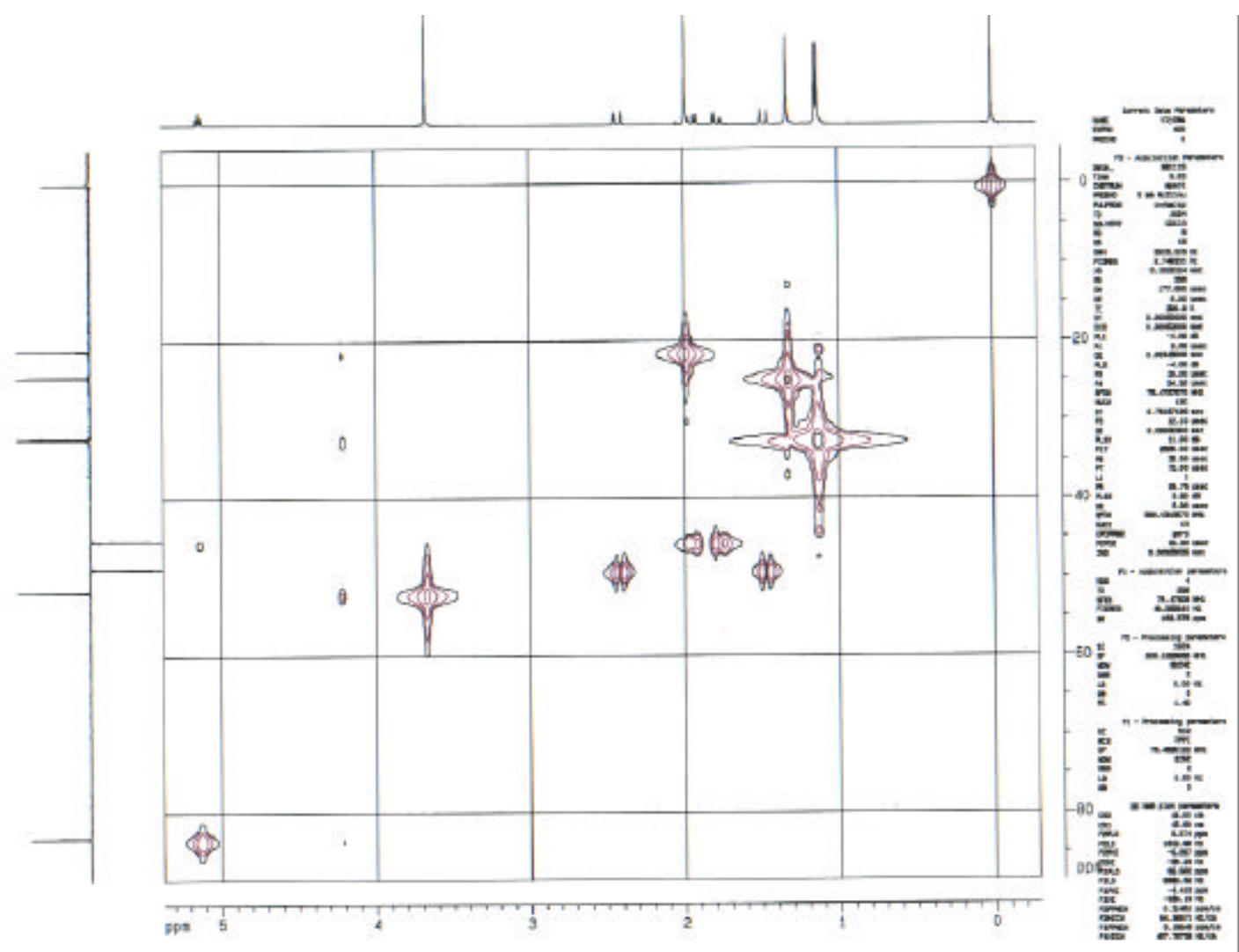

\section{Espectro Jres do composto (9a)}
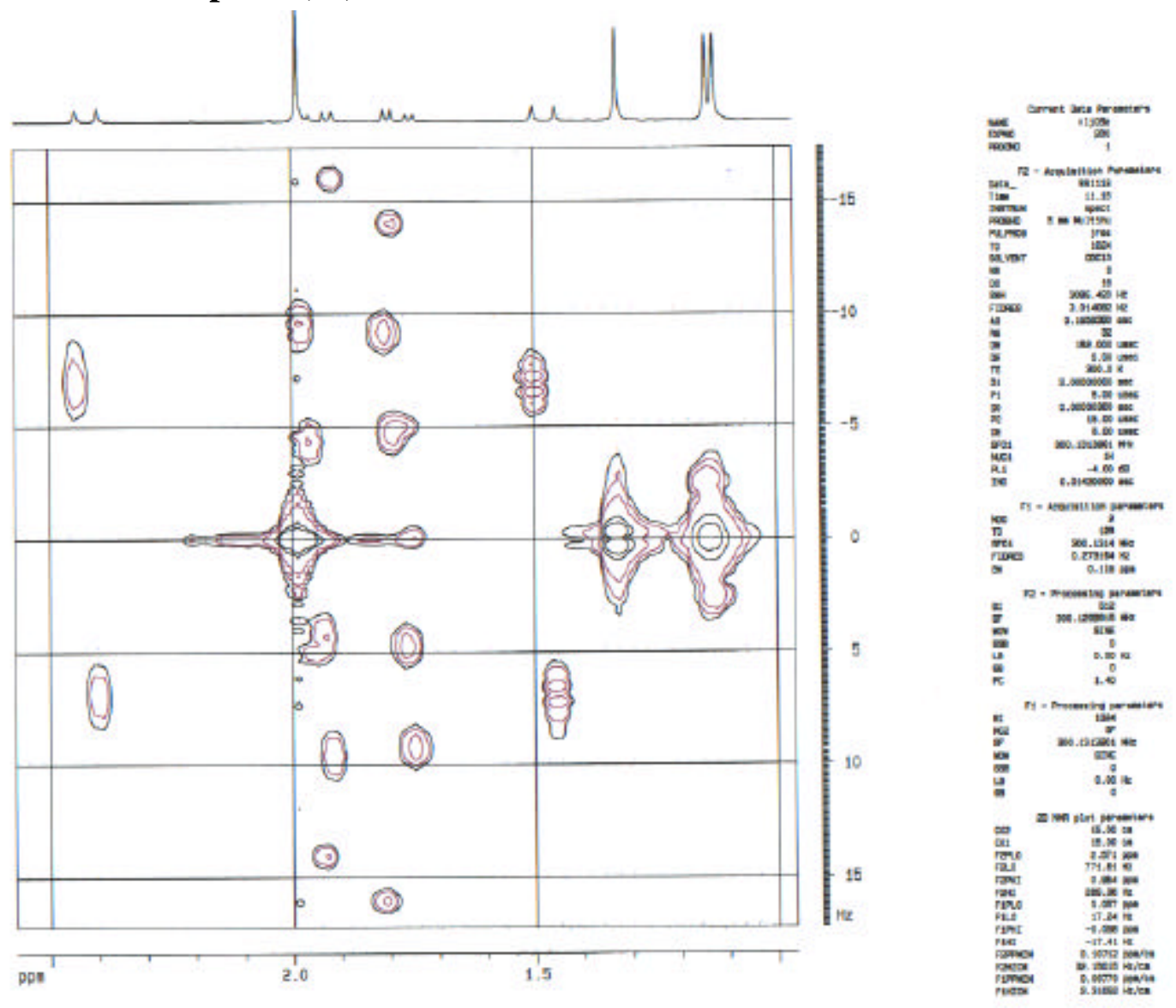


\section{Espectros NOE DIFF do composto (9a)}

$>$ irradiação na metila 6

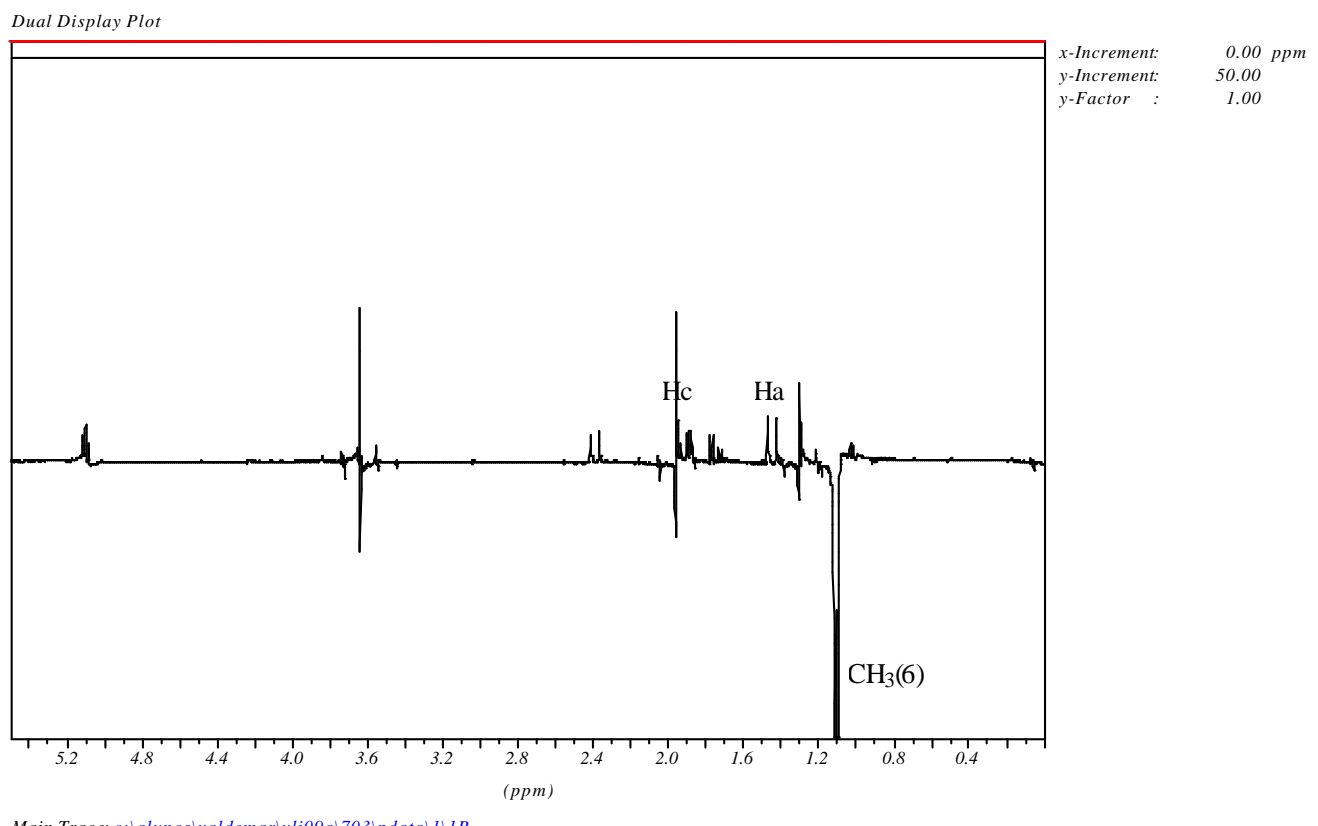

Main Trace: $c: \backslash a l u n o s \backslash$ valdemar $\backslash v l j 09 a \backslash 703 \backslash p d a t a \backslash \backslash \backslash R$ Second Trace: $\backslash W I N I D \backslash S P C \backslash A S P X 32 \backslash V L J 09 A \backslash 704001.1 R$

irradiação na metila 7

Dual Display Plot

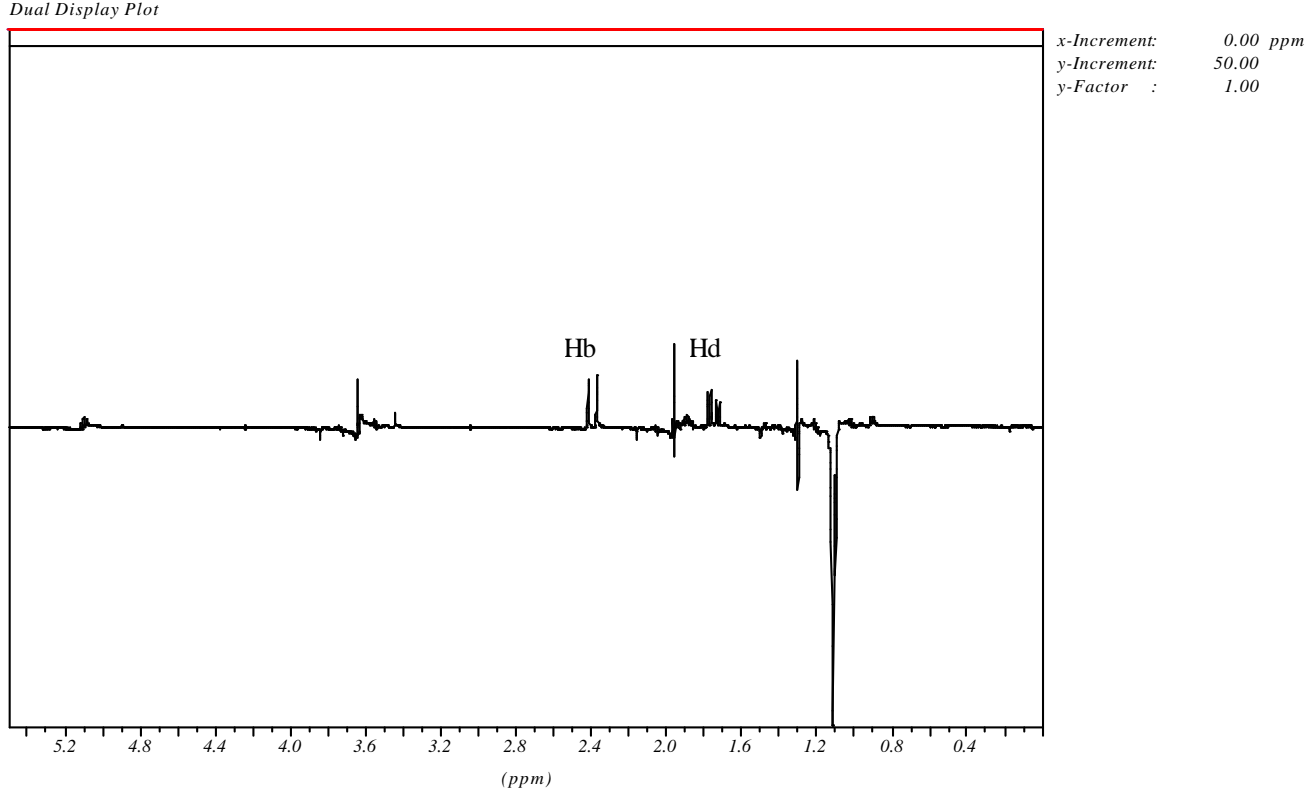

Main Trace: $c$ : $\backslash a$ alunos $\backslash$ valdemar $\backslash v l j 09 a \backslash 702 \backslash p d a t a \backslash I \backslash I R$ Second Trace: $\backslash$ WINID $\backslash S P C \backslash A S P X 32 \backslash V L J 09 A \backslash 704001.1 R$ 
irradiação na metila 8

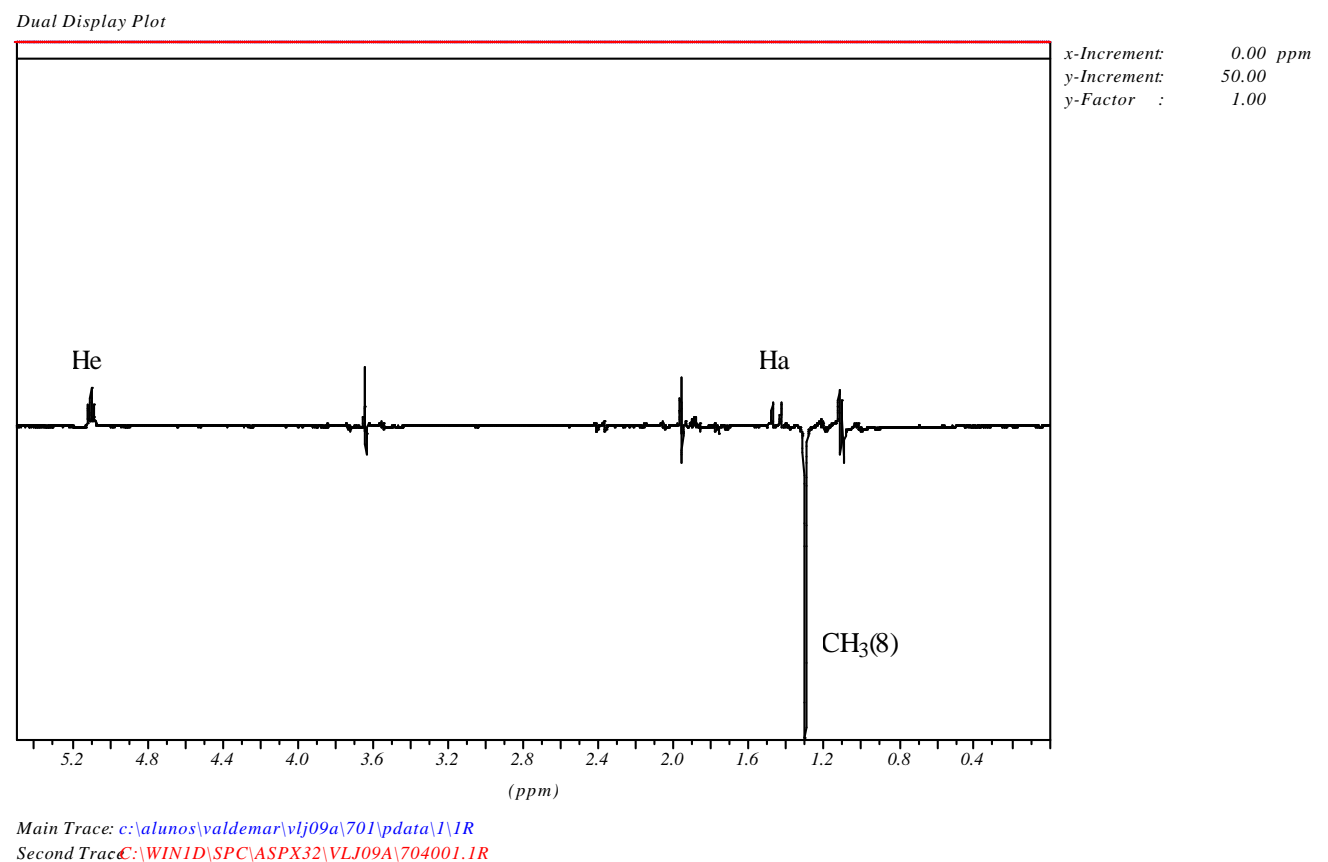

Tabela 35 - Dados espectrais de NOE DIFF do composto 9a $\left(\mathrm{CDCl}_{3}\right)$.

\begin{tabular}{c|c|c}
$\delta$ (ppm)Irradiado & Atribuição & Efeito NOE Observado \\
& & \\
1,32 & $\mathrm{CH}_{3}(8)$ & $\mathrm{Ha}, \mathrm{He}$ \\
1,14 & $\mathrm{CH}_{3}(7)$ & $\mathrm{Hb}, \mathrm{Hd}$ \\
1,12 & $\mathrm{CH}_{3}(6)$ & $\mathrm{Ha}, \mathrm{Hc}$ \\
\hline
\end{tabular}

Tabela 36 - Dados espectrais de ${ }^{1} \mathrm{H}$ e ${ }^{13} \mathrm{C}$ RMN para o $( \pm)$-cis-2-acetoxi-1,4,4trimetil-ciclopentano-1-carboxilato de metila $\left(\mathrm{CDCl}_{3}\right)$

\begin{tabular}{|c|c|c|}
\hline $\mathrm{C}-, \mathrm{H}-$ & ${ }^{1} \mathrm{H}(300 \mathrm{MHz}) \delta(\mathrm{ppm})$ & ${ }^{13} \mathrm{C}(75 \mathrm{MHz})$ \\
\hline 1 & $\mathrm{He}-5,13(\mathrm{t}, 1 \mathrm{H}, \mathrm{Jec}=\mathrm{Jed}=5 \mathrm{~Hz})$ & 83,04 \\
\hline 2 & $\begin{array}{c}\mathrm{Hc}-1,95(\mathrm{Hdd}, \mathrm{Jcd}=14 ; \mathrm{Jce}=5 \text { e Jca }=0,7 \mathrm{~Hz}) \text { e } \mathrm{Hd}-1,78 \\
(\mathrm{dd}, 1 \mathrm{H}, \mathrm{Jdc}=14 \text { e Jde=5Hz) }\end{array}$ & 45,26 \\
\hline 3 & & 36,71 \\
\hline 4 & $\begin{array}{c}\mathrm{Hb}-2,42(\mathrm{~d}, 1 \mathrm{H}, \mathrm{Jba}=13,8 \mathrm{~Hz}) \mathrm{e} \mathrm{Ha}-1,47(\mathrm{dd}, 1 \mathrm{H}, \mathrm{Jab}=13,8 \mathrm{e} \\
\mathrm{Jac}=0,7 \mathrm{~Hz})\end{array}$ & 48,82 \\
\hline 5 & & 54,26 \\
\hline 6 & $1,12(\mathrm{~s}, 3 \mathrm{H})$ & $32,27^{*}$ \\
\hline 7 & $1,14(\mathrm{~s}, 3 \mathrm{H})$ & $32,07^{*}$ \\
\hline 8 & $1,32(\mathrm{~s}, 3 \mathrm{H})$ & 24,41 \\
\hline 9 & & 175,35 \\
\hline 10 & $3,67(\mathrm{~s}, 3 \mathrm{H})$ & 51,75 \\
\hline 11 & & 170,07 \\
\hline 12 & $1,98(\mathrm{~s}, 3 \mathrm{H})$ & 21,12 \\
\hline
\end{tabular}

*Podem estar invertidos 


\section{Composto (9b)}

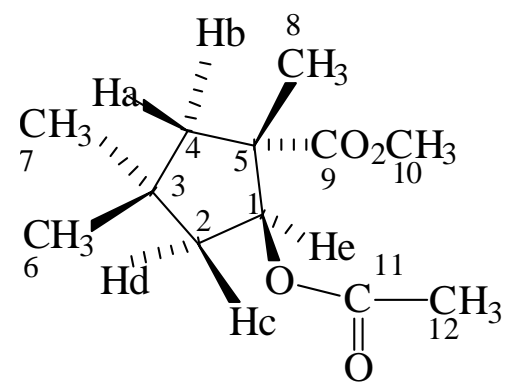

\section{Espectro de ${ }^{1} \mathrm{H}-\mathrm{RMN}$ do composto $(9 \mathrm{~b})$}

\section{9b}

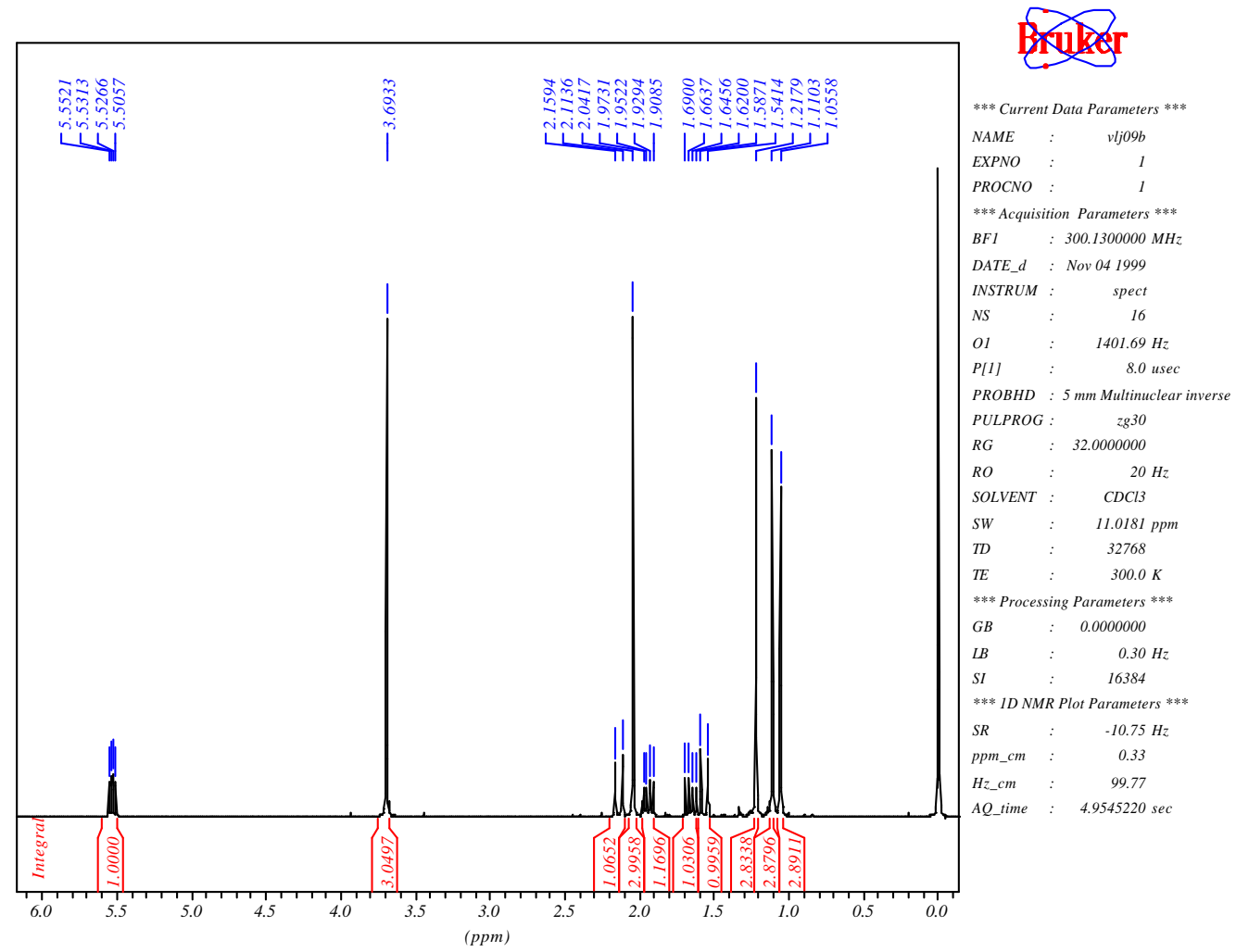

Tabela 37 - Dados espectrais de ${ }^{1} \mathrm{H}$ RMN do composto $9 \mathbf{b}\left(\mathrm{CDCl}_{3}\right)$.

\begin{tabular}{c|l|l|c}
\hline$\delta(\mathbf{p p m})$ & Atribuição & sinal & J (Hz) \\
\hline 5,53 & $\mathrm{He}$ & $\mathrm{dd}$ & $\mathrm{Jec}=7,6$ e Jed $=6,3 \mathrm{~Hz}$ \\
3,69 & $\mathrm{OCH}_{3}(10)$ & $\mathrm{s}$ & \\
2,14 & $\mathrm{Hb}$ & $\mathrm{dd}$ & $\mathrm{Jba}=13,7$ e Jbd=1,4 Hz \\
2,04 & $\mathrm{CH}_{3}(12)$ & $\mathrm{s}$ & $\mathrm{Jdc}=13,2 ; \mathrm{Jde}=6,3$ e Jdb $=1,4$ \\
1,94 & $\mathrm{Hd}$ & $\mathrm{ddd}$ & $\mathrm{Hz}$ \\
& & & $\mathrm{Jcd}=13,2 ; \mathrm{Jce}=7,6 \mathrm{~Hz}$ \\
1,65 & $\mathrm{Hc}$ & $\mathrm{dd}$ & $\mathrm{Jab}=13,7 \mathrm{~Hz}$ \\
1,56 & $\mathrm{Ha}$ & $\mathrm{d}$ & \\
1,36 & $\mathrm{CH}_{3}(8)$ & $\mathrm{s}$ & $\mathrm{s}$ \\
1,14 & $\mathrm{CH}_{3}(7)$ & $\mathrm{s}$ & \\
1,13 & $\mathrm{CH}_{3}(6)$ & &
\end{tabular}




\section{Espectro de ${ }^{13}$ C-RMN (BB) do composto (9b)}

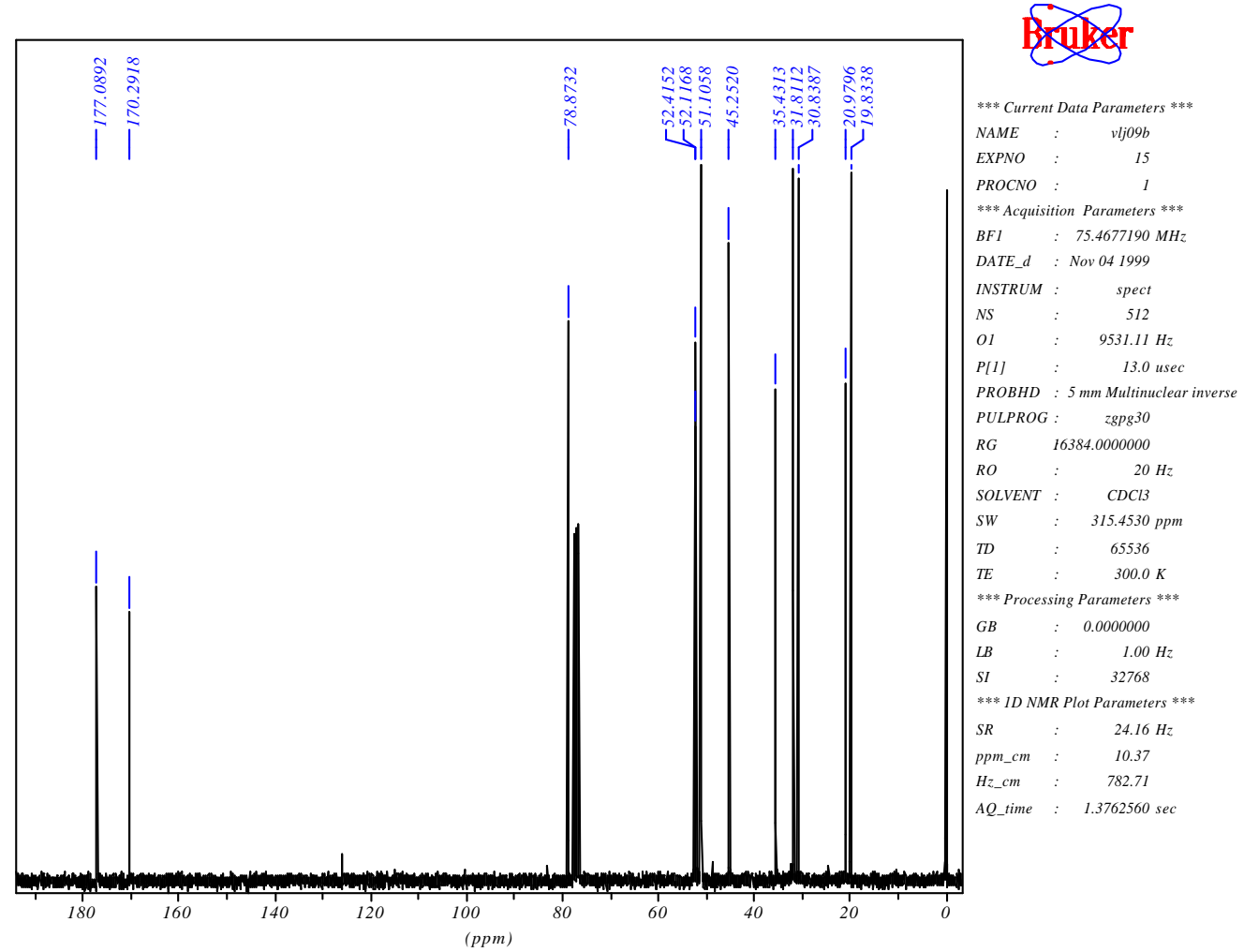

\section{Espectro de ${ }^{13} \mathrm{C}$-RMN (DEPT-135) do composto (9b)}

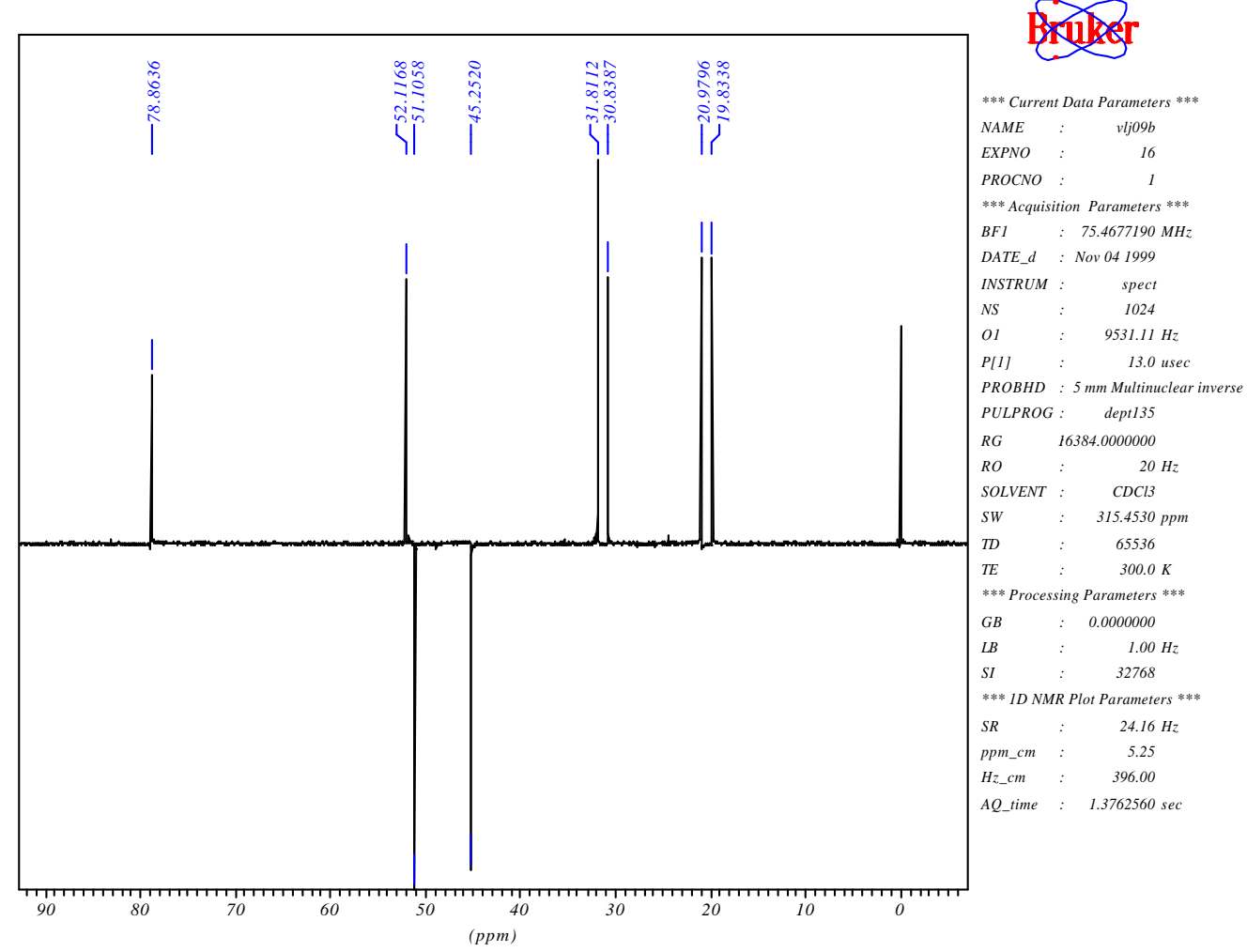




\section{Espectro HMQC (2D H/C) do composto (9b)}

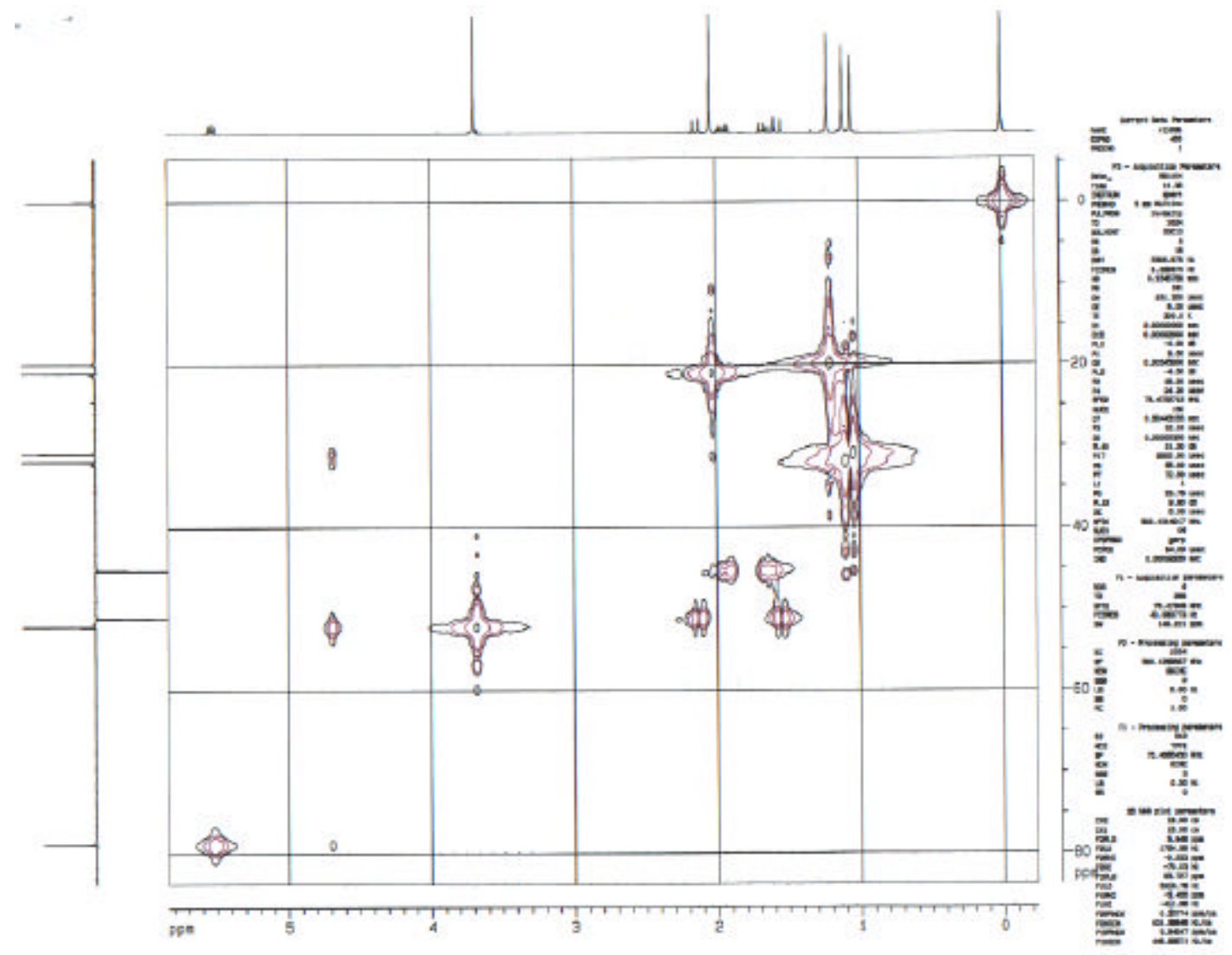

Espectro Jres do composto (9b)
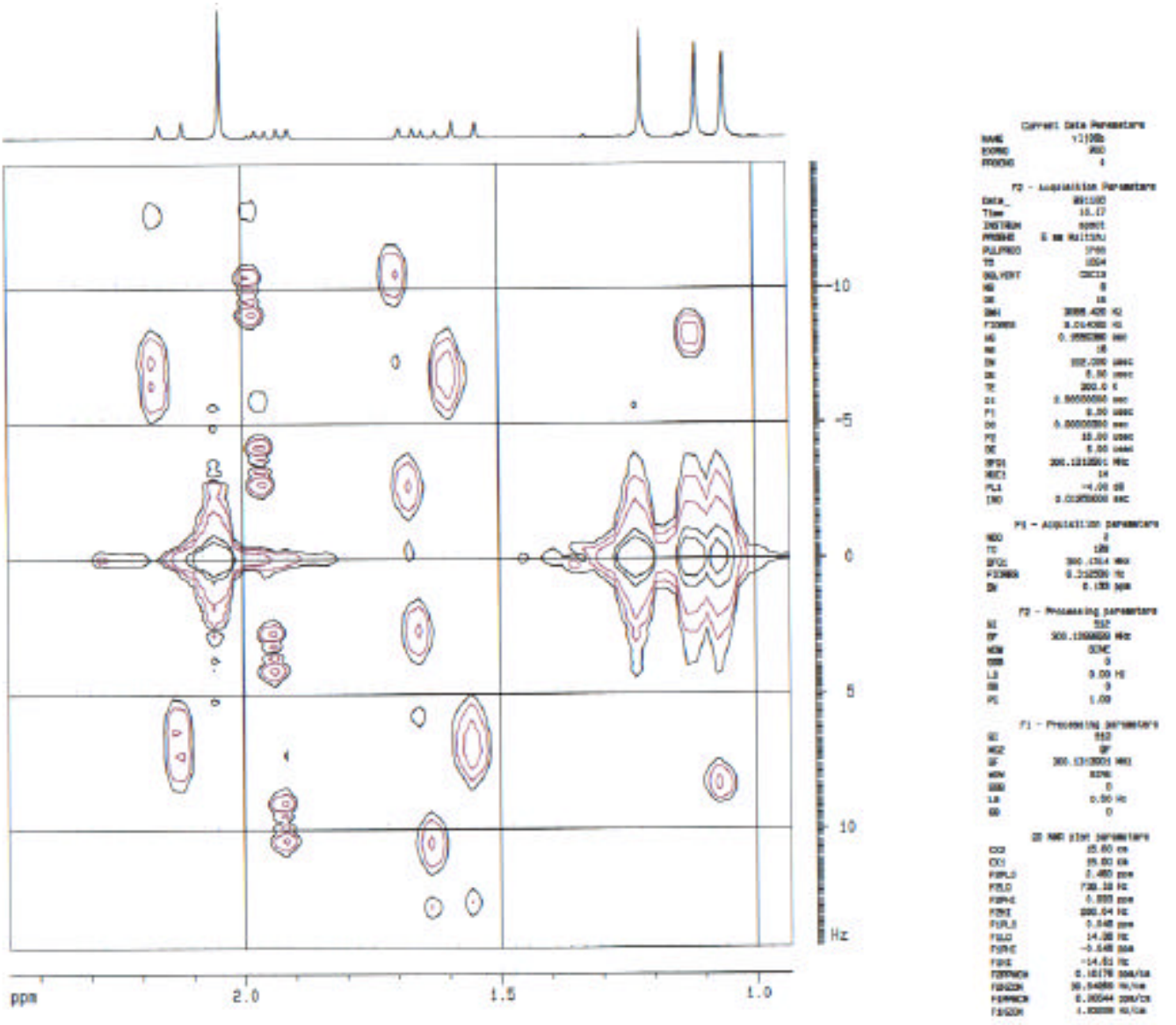


\section{Espectros NOE DIFF do composto (9b)}

$>$ irradiação na metila

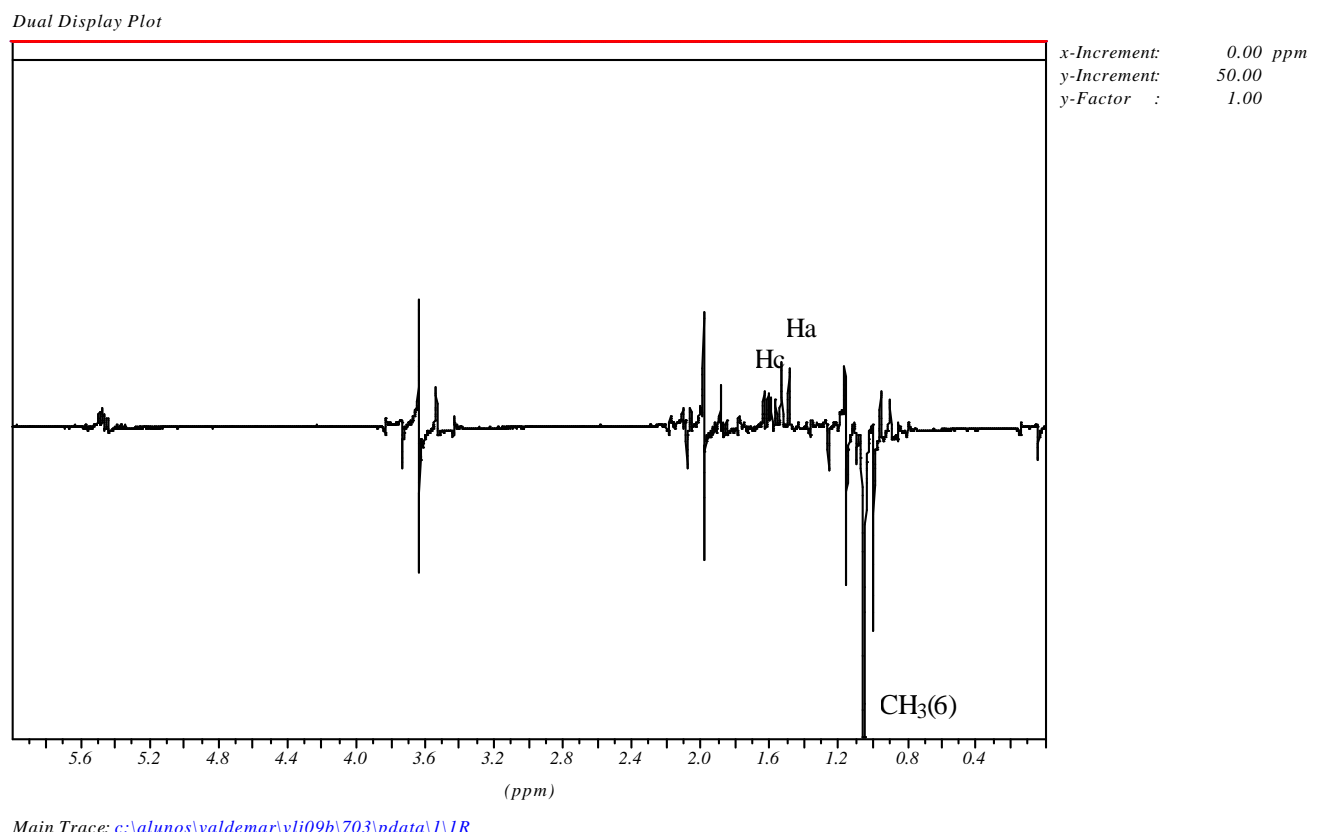

irradiação na metila 7

Dual Display Plot

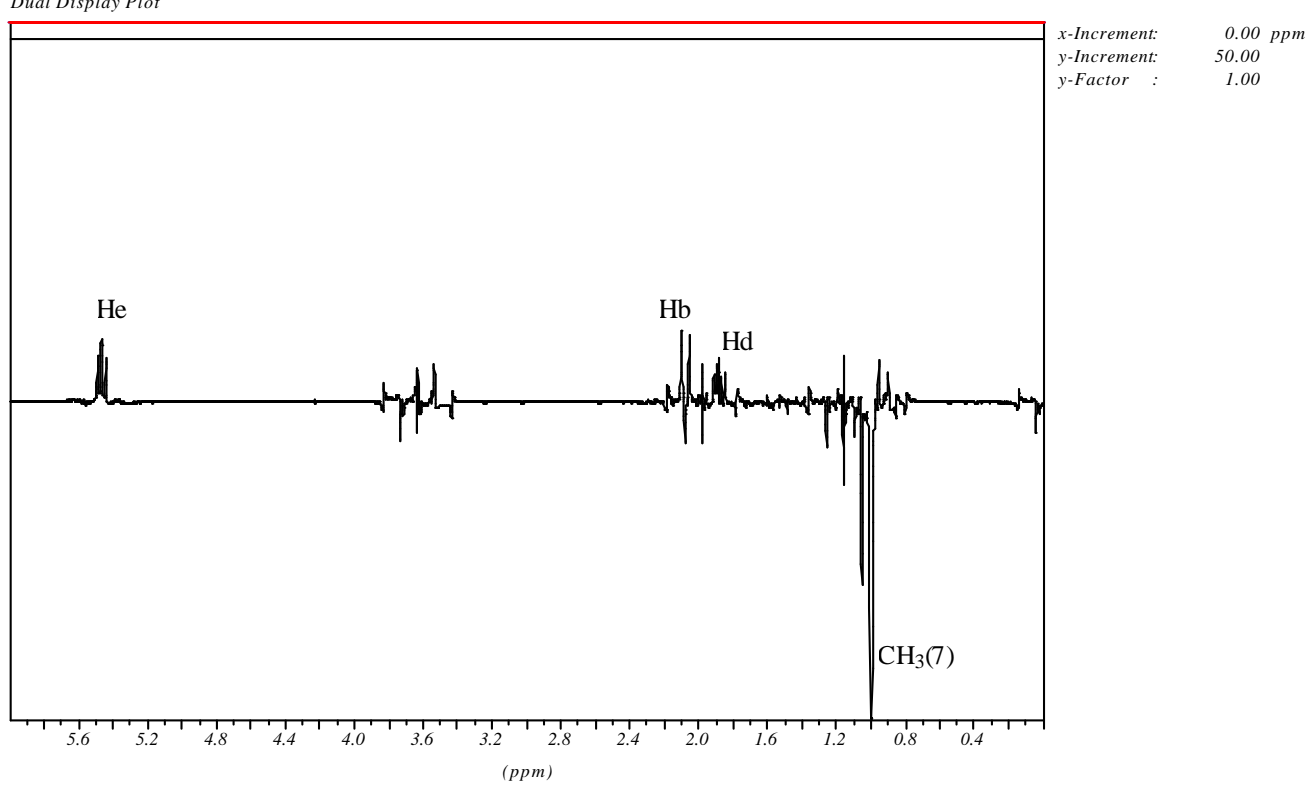

Main Trace: $c: \backslash a$ alunos $\backslash$ valdemar $\backslash v l j 09 b \backslash 704 \backslash p d a t a \backslash I \backslash I R$

Second Trace: $\backslash W I N I D \backslash S P C \backslash A S P X 32 \backslash V L J 09 B \backslash 705001.1 R$ 
irradiação na metila 8

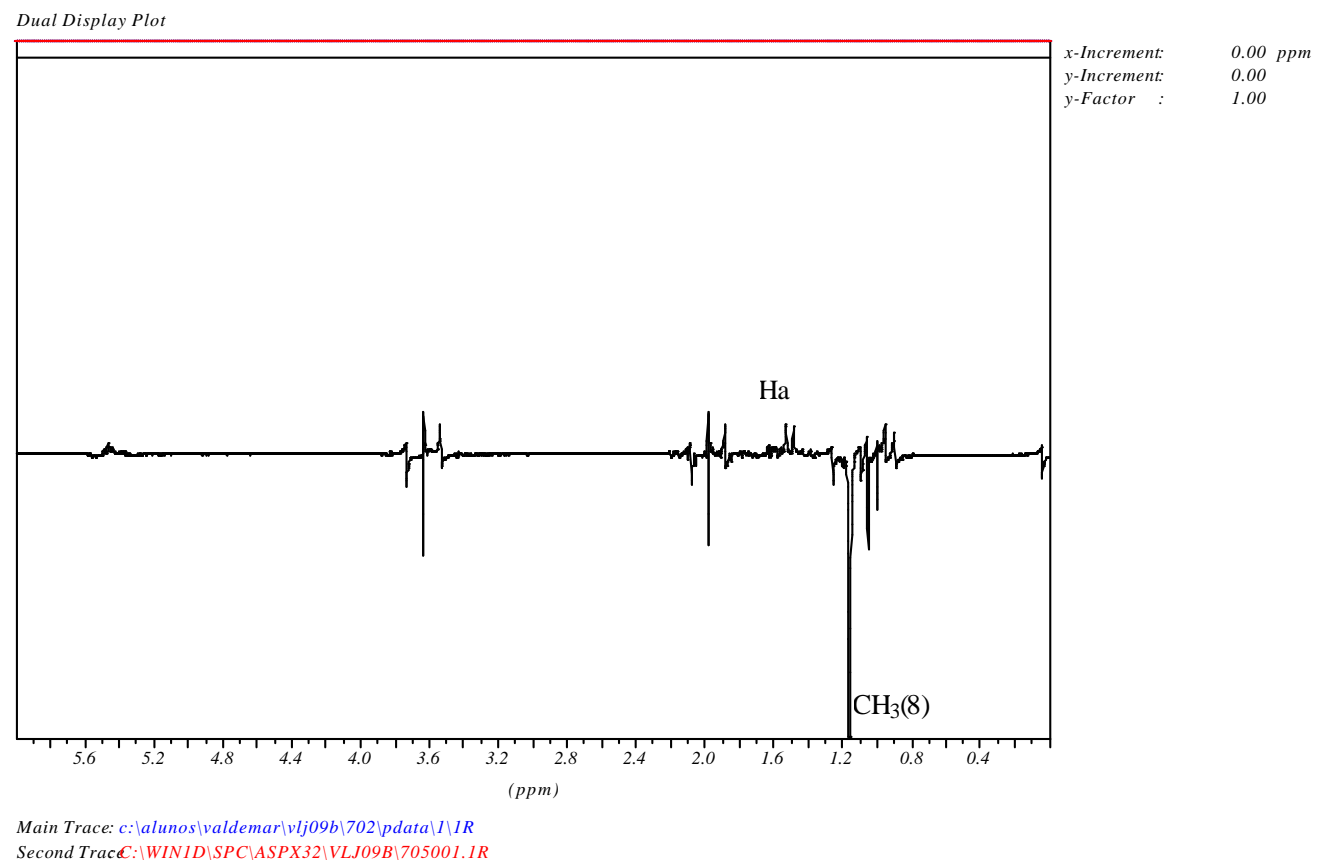

Tabela 38 - Dados espectrais de NOE DIFF do composto $\mathbf{9 b}\left(\mathrm{CDCl}_{3}\right)$.

\begin{tabular}{c|c|c}
$\delta$ (ppm)Irradiado & Atribuição & Efeito NOE Observado \\
1,21 & $\mathrm{CH}_{3}(8)$ & $\mathrm{Ha}$ \\
1,11 & $\mathrm{CH}_{3}(6)$ & $\mathrm{Ha}, \mathrm{Hc}$ \\
1,05 & $\mathrm{CH}_{3}(7)$ & $\mathrm{Hb}, \mathrm{Hd}, \mathrm{He}$ \\
\hline
\end{tabular}

Tabela 39 - Dados espectrais de ${ }^{1} \mathrm{H}$ e ${ }^{13} \mathrm{C}$ RMN para o (士)-trans-2-acetoxi-1,4,4trimetil-ciclopentano-1-carboxilato de metila $\left(\mathrm{CDCl}_{3}\right)$

\begin{tabular}{|c|c|c|}
\hline $\mathrm{C}-, \mathrm{H}-$ & ${ }^{1} \mathrm{H}(300 \mathrm{MHz}) \delta(\mathrm{ppm})$ & ${ }^{13} \mathrm{C}(75 \mathrm{MHz})$ \\
\hline 1 & $\mathrm{He}-5,53(\mathrm{dd}, 1 \mathrm{H}, \mathrm{Jec}=7,6$ e Jed=6,3 Hz) & 78,87 \\
\hline 2 & $\begin{array}{c}\mathrm{Hd}-1,94(\mathrm{ddd}, 1 \mathrm{H}, \mathrm{Jdc}=13,2 ; \mathrm{Jde}=6,3 \mathrm{e} \mathrm{Jdb}=1,4 \mathrm{~Hz}) \text { e Hc- } \\
1,65(\mathrm{dd}, 1 \mathrm{H}, \mathrm{Jcd}=13,2 ; \mathrm{Jce}=7,6 \mathrm{~Hz})\end{array}$ & 45,25 \\
\hline 3 & & 35,43 \\
\hline 4 & $\mathrm{Hb}-2,14(\mathrm{dd}, 1 \mathrm{H}, \mathrm{Jba}=13,7 \mathrm{e} \mathrm{Jbd}=1,4 \mathrm{~Hz})$ e Ha-1,56 (d, 1H, \\
& $\mathrm{Jab}=13,7 \mathrm{~Hz})$ & 51,10 \\
\hline 5 & & 52,41 \\
\hline 6 & $1,11(\mathrm{~s}, 3 \mathrm{H})$ & 31,81 \\
\hline 7 & $1,05(\mathrm{~s}, 3 \mathrm{H})$ & 30,83 \\
\hline 8 & $1,21(\mathrm{~s}, 3 \mathrm{H})$ & 19,83 \\
\hline 9 & & 52,11 \\
\hline 10 & $3,69(\mathrm{~s}, 3 \mathrm{H})$ & 170,29 \\
\hline 11 & & 20,97 \\
\hline 12 & $2,04(\mathrm{~s}, 3 \mathrm{H})$ & \\
\hline
\end{tabular}




\section{Composto (10a)}

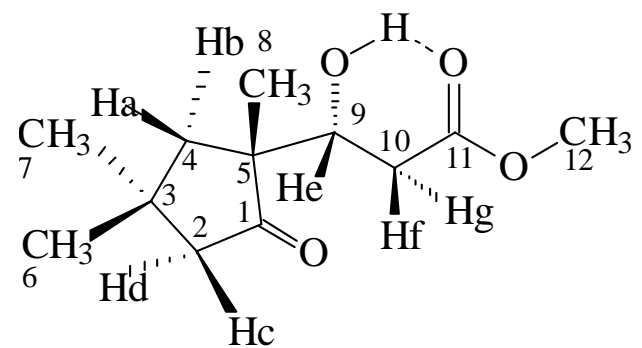

$10 a$

Espectro de ${ }^{1}$ H-RMN do composto (10a)

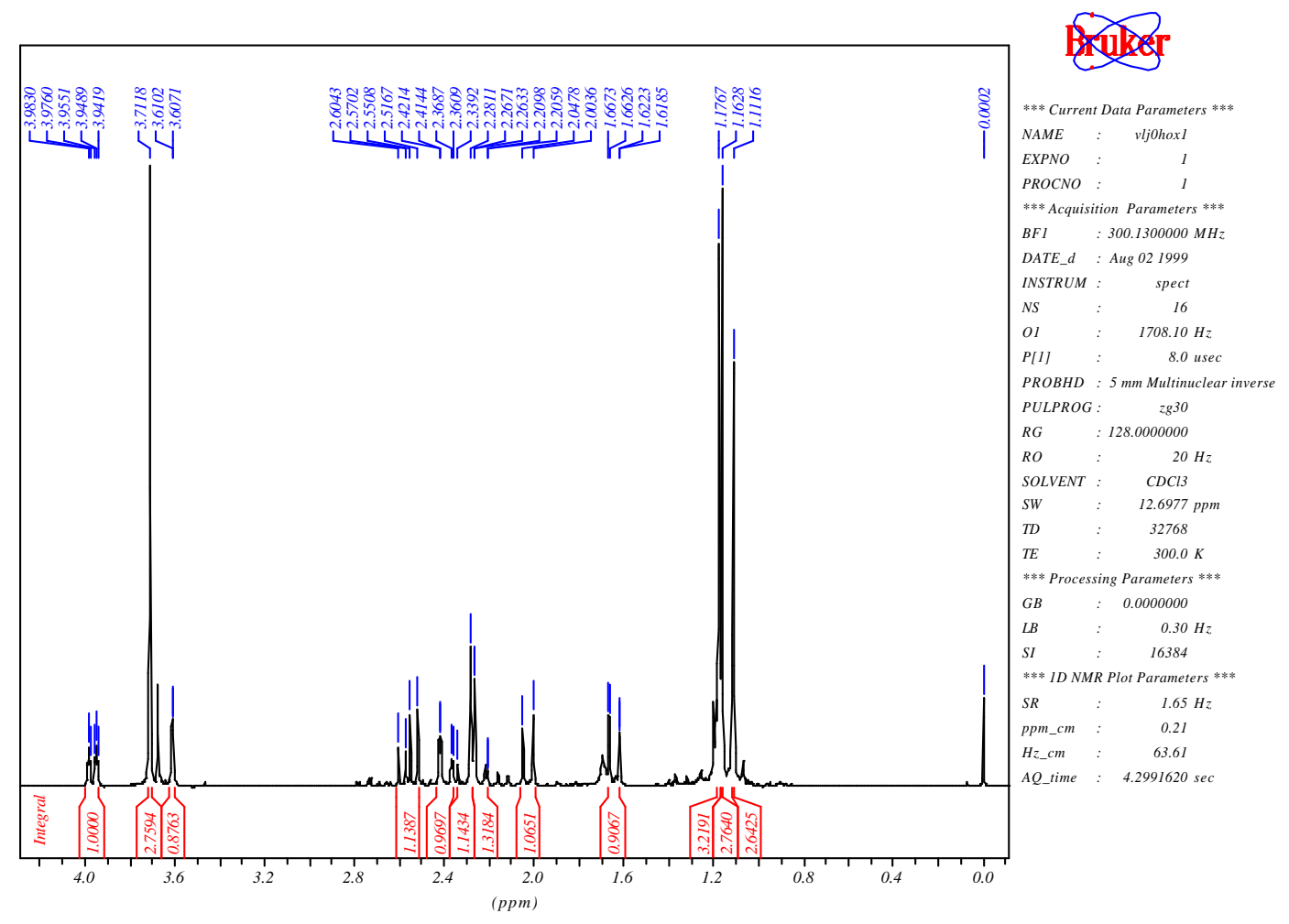

Tabela 40 - Dados espectrais de ${ }^{1} \mathrm{H}$ RMN do composto $\mathbf{1 0 a}\left(\mathrm{CDCl}_{3}\right)$.

\begin{tabular}{c|l|c|c}
\hline$\delta$ (ppm) & Atribuição & sinal & J (Hz) \\
\hline 3,96 & $\mathrm{He}$ & $\mathrm{dt}$ & $\mathrm{Jeg}=10,2 ; \mathrm{Jef}=\mathrm{Je}-\mathrm{OH}=2,1$ \\
& & & $\mathrm{~Hz}$ \\
3,71 & $\mathrm{OCH}_{3}(12)$ & $\mathrm{s}$ & $\mathrm{J}$ \\
3,06 & $\mathrm{OH}$ & $\mathrm{d}$ & $\mathrm{J}-\mathrm{e}=2,1 \mathrm{~Hz}$ \\
2,56 & $\mathrm{Hg}$ & $\mathrm{dd}$ & $\mathrm{Jgf}=16 \mathrm{e} \mathrm{Jge}=10,2 \mathrm{~Hz}$ \\
2,39 & $\mathrm{Hf}$ & $\mathrm{d}$ & $\mathrm{Jfg}=16 \mathrm{e} \mathrm{Jfe}=2,1 \mathrm{~Hz}$ \\
2,31 & $\mathrm{Hd}$ & $\mathrm{dd}$ & $\mathrm{Jdc}=17,4 \mathrm{~Hz}$ \\
2,23 & $\mathrm{Hc}$ & $\mathrm{d}$ & $\mathrm{Jba}=13,3 \mathrm{Jca}=1,2 \mathrm{~Hz}$ \\
2,02 & $\mathrm{Hb}$ & $\mathrm{dd}$ & $\mathrm{Jab}=13,3 \mathrm{e} \mathrm{Jac}=1,2 \mathrm{~Hz}$ \\
1,64 & $\mathrm{Ha}$ & $\mathrm{s}$ & \\
1,17 & $\mathrm{CH}_{3}(7)$ & $\mathrm{s}$ & \\
1,16 & $\mathrm{CH}_{3}(8)$ & $\mathrm{s}$ & \\
1,11 & $\mathrm{CH}_{3}(6)$ & &
\end{tabular}


Espectro de ${ }^{13} \mathrm{C}-\mathrm{RMN}$ (BB) do composto (10a)

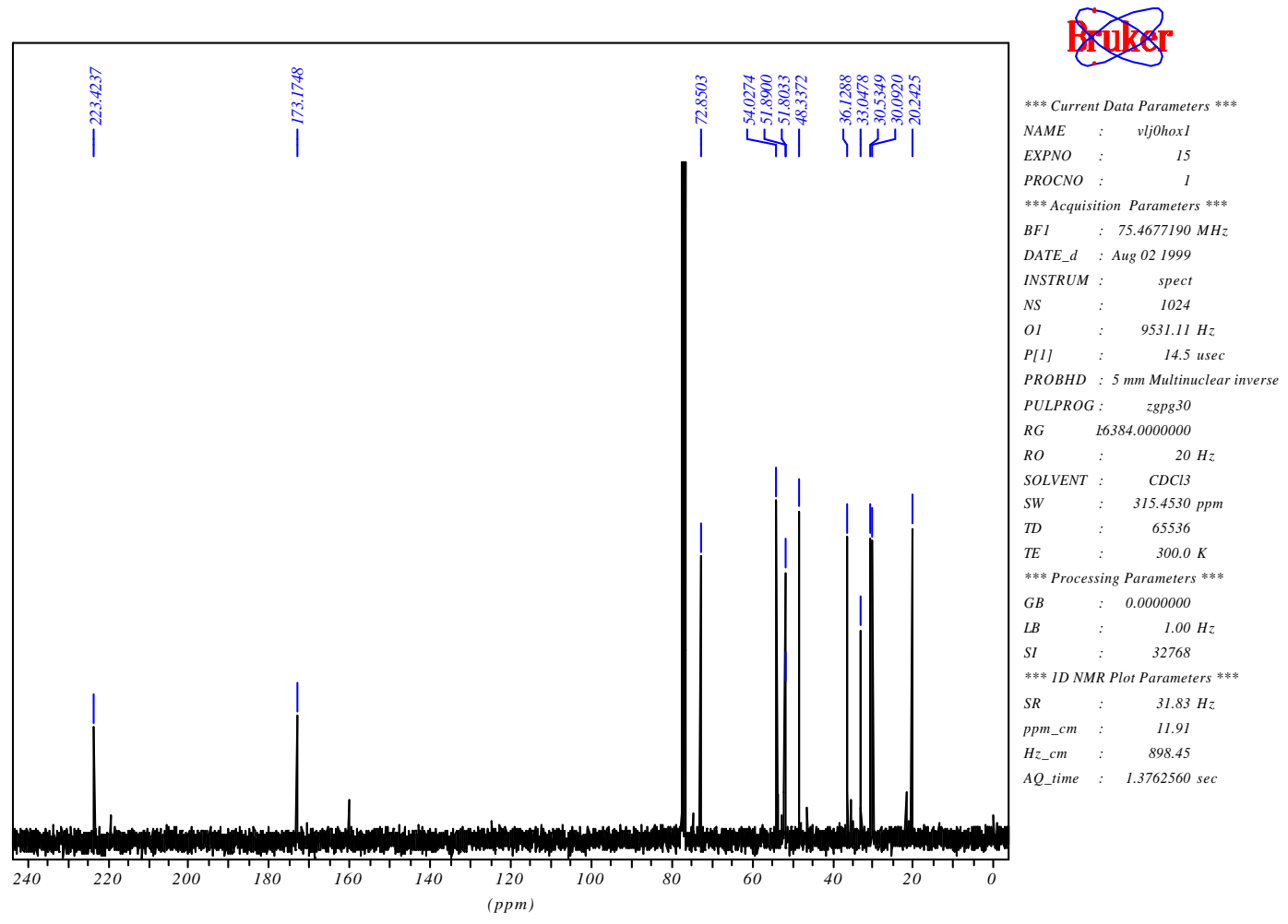

Espectro de ${ }^{13} \mathrm{C}-\mathrm{RMN}$ (DEPT-135) do composto (10a)

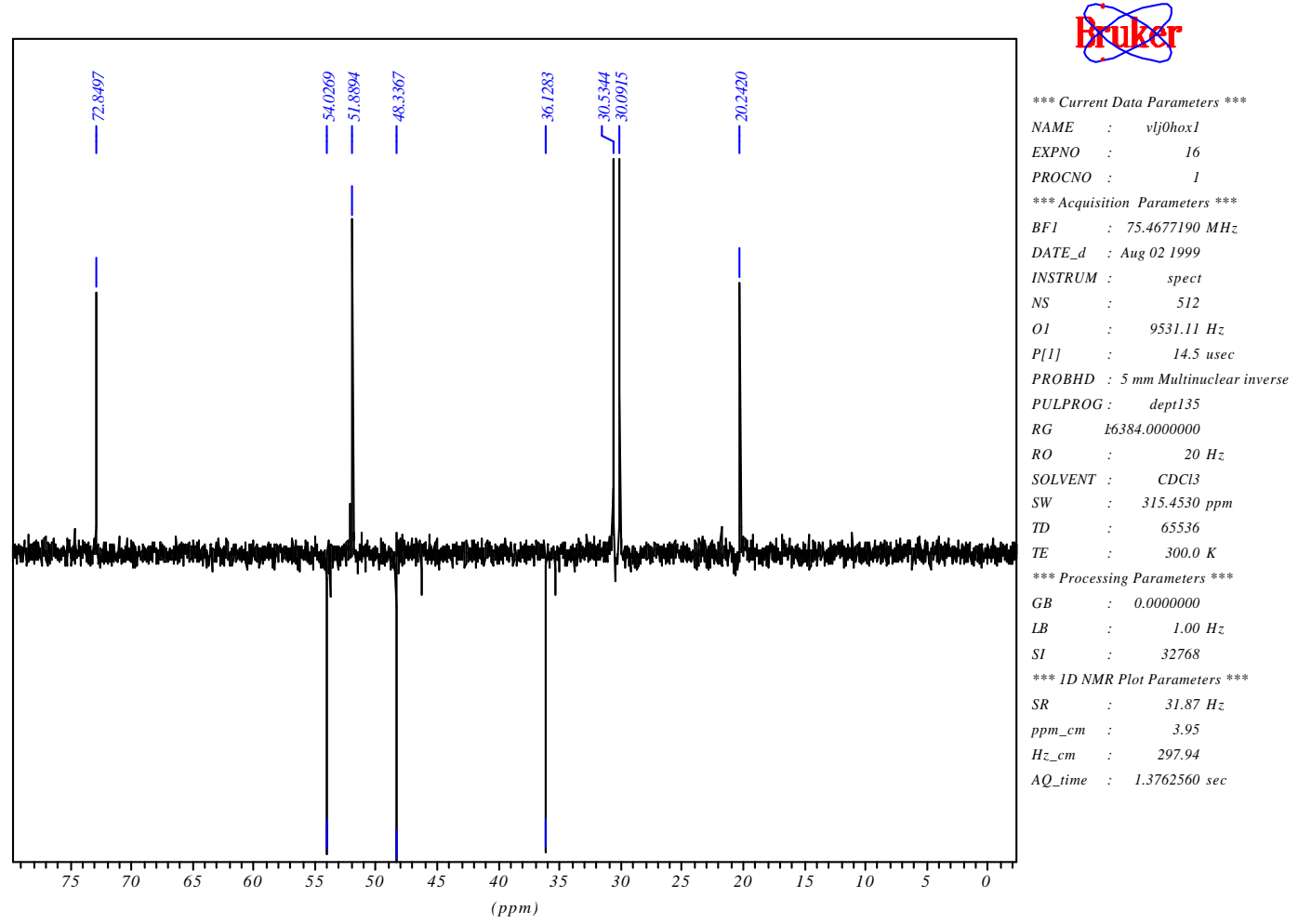




\section{Espectro HMQC (2D H/C) do composto (10a)}

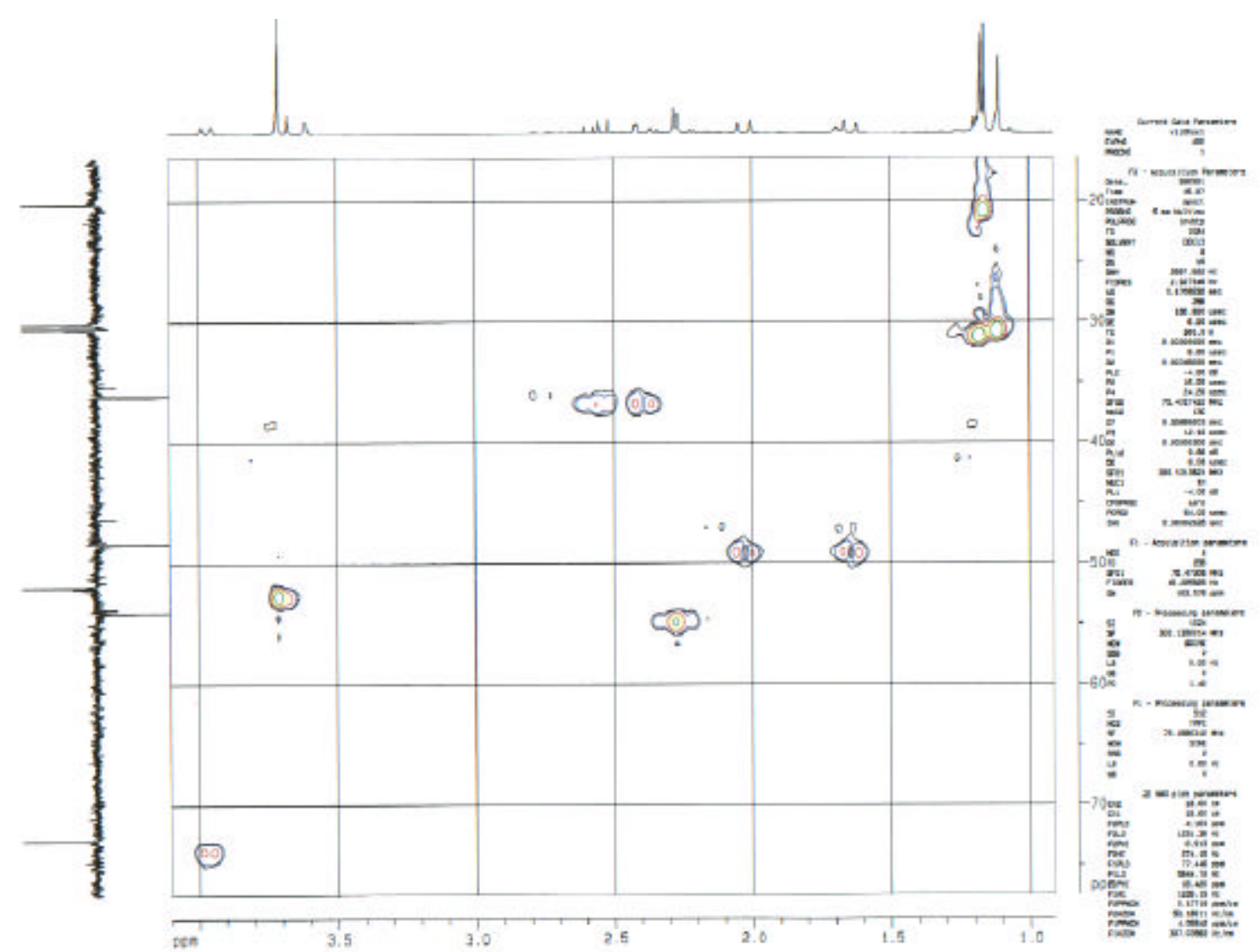

Espectr os NOE DIFF do composto (10a)

$>$ irradiação na metila 6

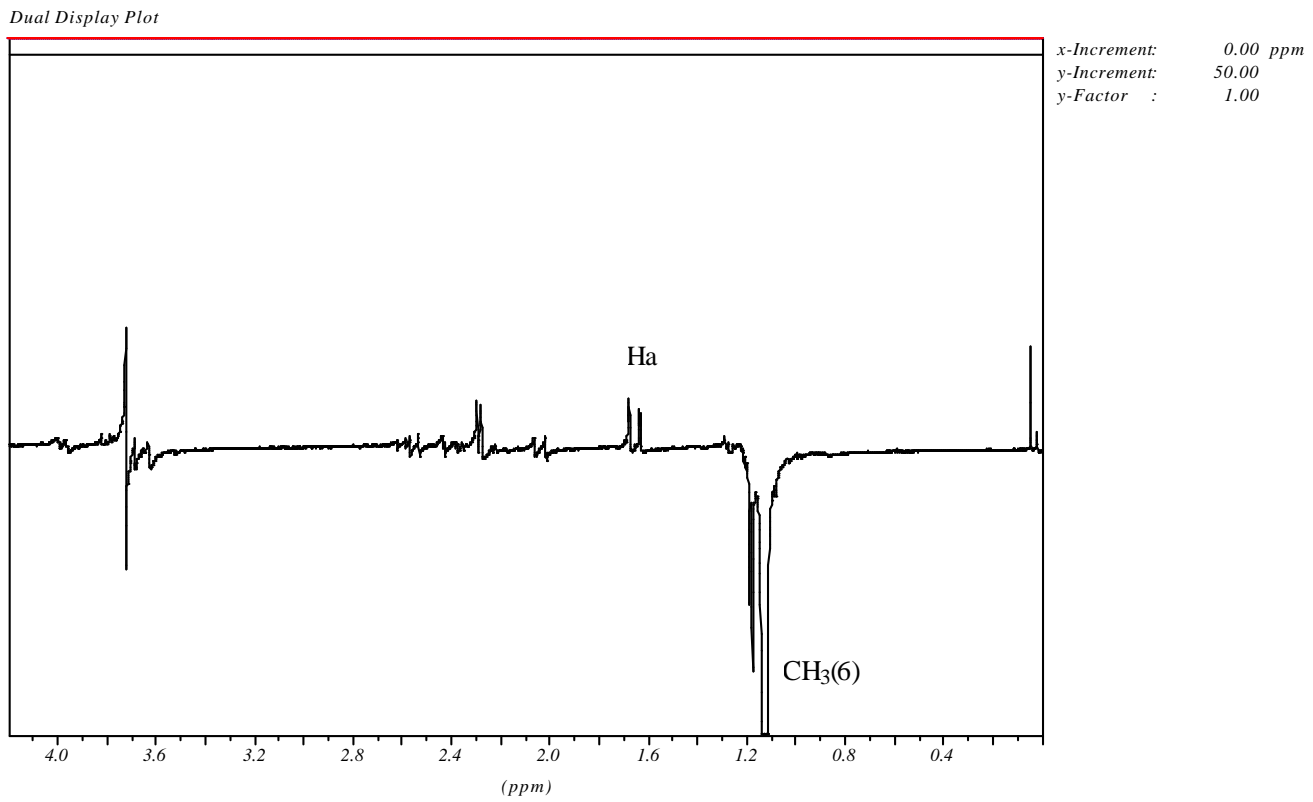

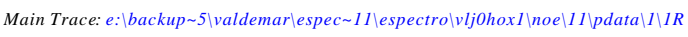

Second Trace: $\backslash$ WINID \SPC\ASPX32\NOE\012001.1R 
irradiação na metila 7

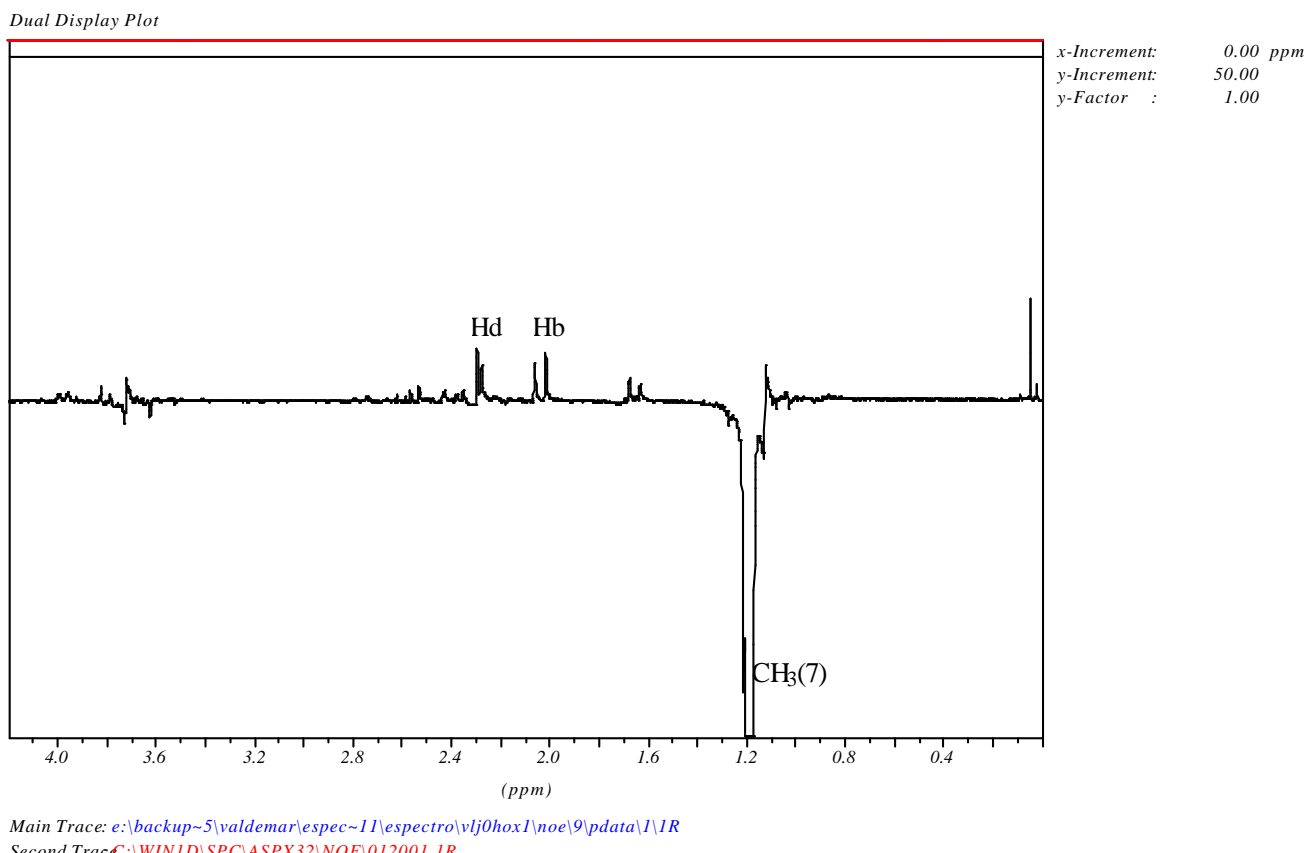

Second Trace: $\backslash$ WINID $\backslash S P C \backslash A S P X 32 \backslash N O E \backslash 012001.1 R$

irradiação na metila 8

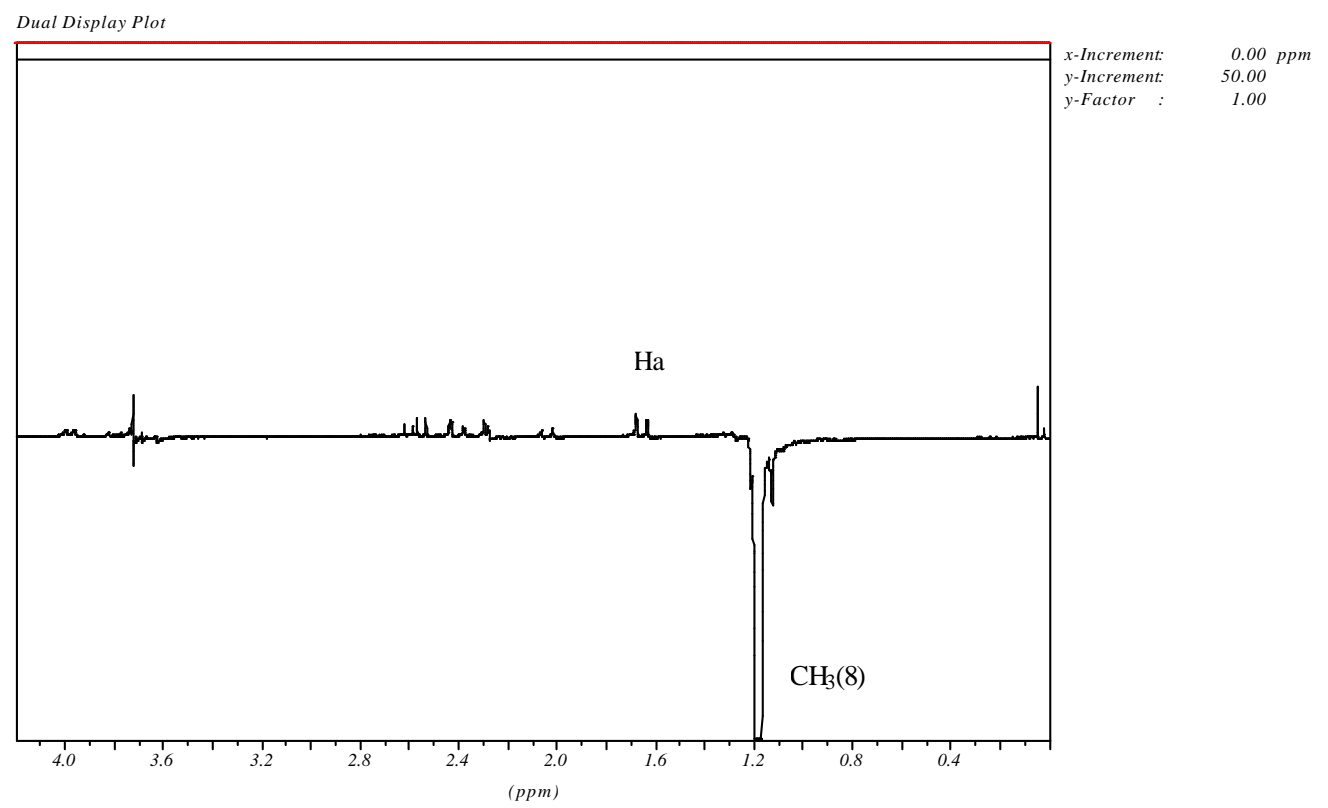

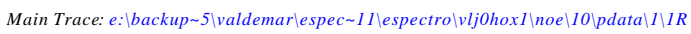

Second TraceC: $\backslash W I N 1 D \backslash S P C \backslash A S P X 32 \backslash N O E \backslash 012001.1 R$ 
Tabela 41 - Dados espectrais de NOE DIFF do composto 10a $\left(\mathrm{CDCl}_{3}\right)$.

\begin{tabular}{c|c|c}
\hline$\delta(\mathbf{p p m})$ Irradiado & Atribuição & Efeito NOE Observado \\
\hline 1,17 & & $\mathrm{Hb}, \mathrm{Hd}$ \\
1,16 & $\mathrm{CH}_{3}(7)$ & $\mathrm{Ha}$ \\
1,11 & $\mathrm{CH}_{3}(8)$ & $\mathrm{Ha}, \mathrm{Hc}$ \\
\hline
\end{tabular}

Tabela 42 - Dados espectrais de ${ }^{1} \mathrm{H}$ e ${ }^{13} \mathrm{C}$ RMN para o rel-[3 $\left.\alpha, 1^{\prime} \beta\right]-( \pm)$ - 3 -Hidroxi3-(1',4',4'-trimetil-2'-oxo-ciclopentil)-propanoato de metila $\left(\mathrm{CDCl}_{3}\right)$

\begin{tabular}{|c|c|c|}
\hline C-, H- & ${ }^{1} \mathrm{H}(300 \mathrm{MHz}) \delta(\mathrm{ppm})$ & ${ }^{13} \mathrm{C}(75 \mathrm{MHz})$ \\
\hline 1 & & 223,42 \\
\hline 2 & $\begin{array}{l}\mathrm{Hd}-2,31(\mathrm{~d}, 1 \mathrm{H}, \mathrm{Jdc}=17,4 \mathrm{~Hz}) \text { e Hc-2,23 (dd, 1H, Jcd=17,4 e } \\
\text { Jca }=1,2 \mathrm{~Hz})\end{array}$ & 54,02 \\
\hline 3 & & 33,04 \\
\hline 4 & $\begin{array}{l}\mathrm{Hb}-2,02(\mathrm{~d}, 1 \mathrm{H}, \mathrm{Jba}=13,3 \mathrm{~Hz}) \text { e } \mathrm{Ha}-1,64(\mathrm{dd}, 1 \mathrm{H}, \mathrm{Jab}=13,3 \mathrm{e} \\
\mathrm{Jac}=1,2 \mathrm{~Hz})\end{array}$ & 48,33 \\
\hline 5 & & 51,80 \\
\hline 6 & $1,11(\mathrm{~s}, 3 \mathrm{H})$ & 30,09 \\
\hline 7 & $1,17(\mathrm{~s}, 3 \mathrm{H})$ & 30,53 \\
\hline 8 & $1,16(\mathrm{~s}, 3 \mathrm{H})$ & 20,24 \\
\hline 9 & $\begin{array}{c}\mathrm{He}-3,96(\mathrm{dt}, 1 \mathrm{H}, \mathrm{Jeg}=10,2 ; \mathrm{Jef}=\mathrm{Je}-\mathrm{OH}=2,1 \mathrm{~Hz}) \text { e } 3,06(\mathrm{~d}, 1 \mathrm{H}, \\
\mathrm{OH}, \mathrm{JOH}-\mathrm{e}=2,1 \mathrm{~Hz})\end{array}$ & 72,85 \\
\hline $10 *$ & $\begin{array}{c}\mathrm{Hg}-2,56(\mathrm{dd}, 1 \mathrm{H}, \mathrm{Jgf}=16 \text { e Jge }=10,2 \mathrm{~Hz}) \text { e Hf-2,39 (dd, 1H, } \\
\text { Jfg=16 e Jfe }=2,1 \mathrm{~Hz})\end{array}$ & 36,12 \\
\hline 11 & & 173,17 \\
\hline 12 & $3,71(\mathrm{~s}, 3 \mathrm{H})$ & 51,89 \\
\hline
\end{tabular}

* A atribuição dos sinais $\mathrm{Hg}$ e $\mathrm{Hf}$ foi feita baseado em estudos conformacionais realizados no programa PC Model 7. 


\section{Composto (10b)}

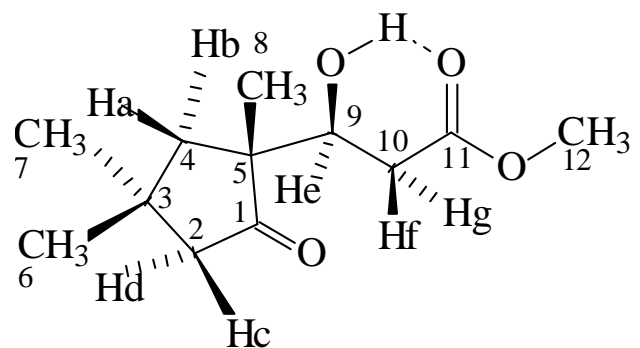

10b

\section{Espectro de ${ }^{1} \mathrm{H}$-RMN do composto (10b)}

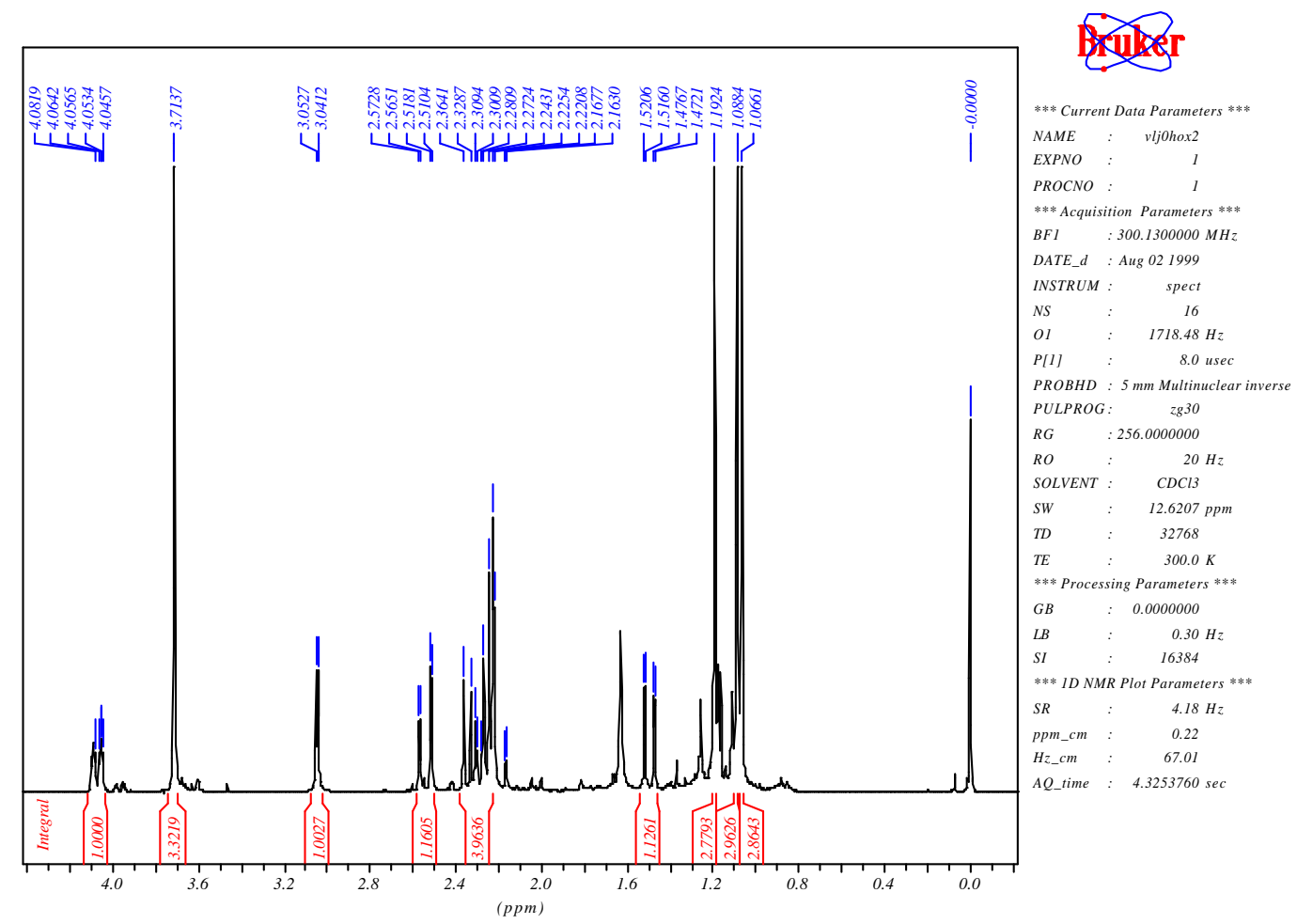

Tabela 43 - Dados espectrais de ${ }^{1} \mathrm{H}$ RMN do composto $\mathbf{1 0 b}\left(\mathrm{CDCl}_{3}\right)$.

\begin{tabular}{|c|c|c|c|}
\hline$\delta($ ppm) & Atribuição & sinal & $\overline{\mathrm{J}(\mathbf{H z})}$ \\
\hline $\begin{array}{c}4,07 \\
3,71 \\
3,05 \\
2,54 \\
2,32 \\
2,27 \\
2,24 \\
2,19 \\
1,5 \\
1,19 \\
1,08 \\
1,06 \\
\end{array}$ & $\begin{array}{l}\mathrm{He} \\
\mathrm{OCH}_{3}(12) \\
\mathrm{OH} \\
\mathrm{Hg} \\
\mathrm{Hf} \\
\mathrm{Hd} \\
\mathrm{Hb} \\
\mathrm{Hc} \\
\mathrm{Ha} \\
\mathrm{CH}_{3}(7) \\
\mathrm{CH}_{3}(6) \\
\mathrm{CH}_{3}(8) \\
\end{array}$ & $\begin{array}{l}\mathrm{ddd} \\
\mathrm{s} \\
\mathrm{d} \\
\mathrm{dd} \\
\mathrm{dd} \\
\mathrm{d} \\
\mathrm{d} \\
\mathrm{dd} \\
\mathrm{dd} \\
\mathrm{s} \\
\mathrm{s} \\
\mathrm{s} \\
\end{array}$ & $\begin{array}{c}\text { Jef }=10,7 ; \text { Je-OH }=3,1 \text { e Jeg }=2,3 \\
\\
\text { JoH-e }=3,1 \mathrm{~Hz} \\
\text { Jgf }=16,5 \text { e Jeg }=2,3 \mathrm{~Hz} \\
\mathrm{Jfg}=16,5 \text { e Jfe }=10,7 \mathrm{~Hz} \\
\mathrm{Jdc}=17,3 \mathrm{~Hz} \\
\mathrm{Jba}=13,2 \mathrm{~Hz} \\
\mathrm{Jcd}=17,3 \text { e Jca }=1,4 \mathrm{~Hz} \\
\mathrm{Jab}=13,2 \text { e Jac }=1,4 \mathrm{~Hz}\end{array}$ \\
\hline
\end{tabular}


Espectro de ${ }^{13} \mathrm{C}-\mathrm{RMN}$ (BB) do composto (10b)

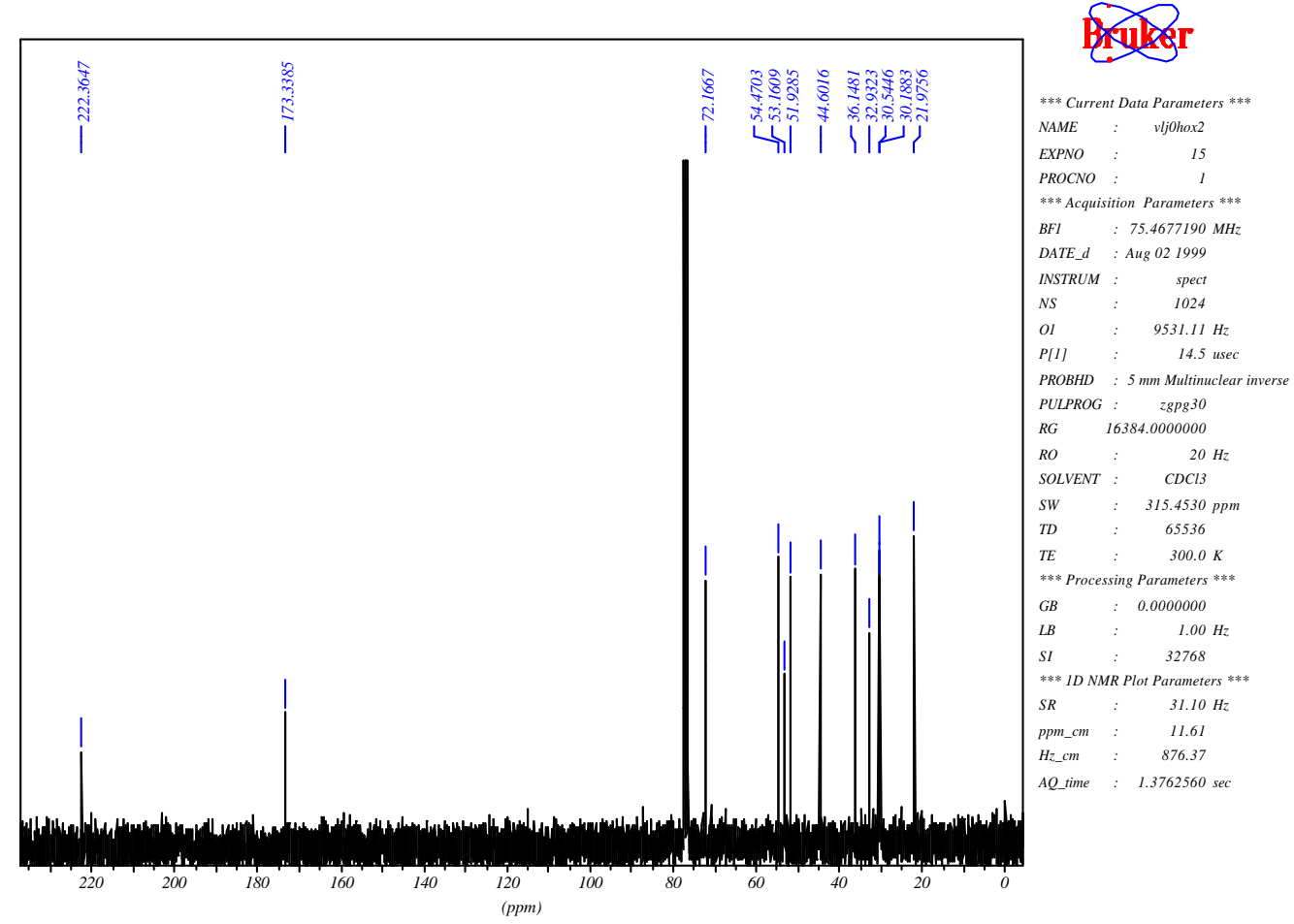

Espectro de ${ }^{13} \mathrm{C}$-RMN (DEPT-135) do composto (10b)

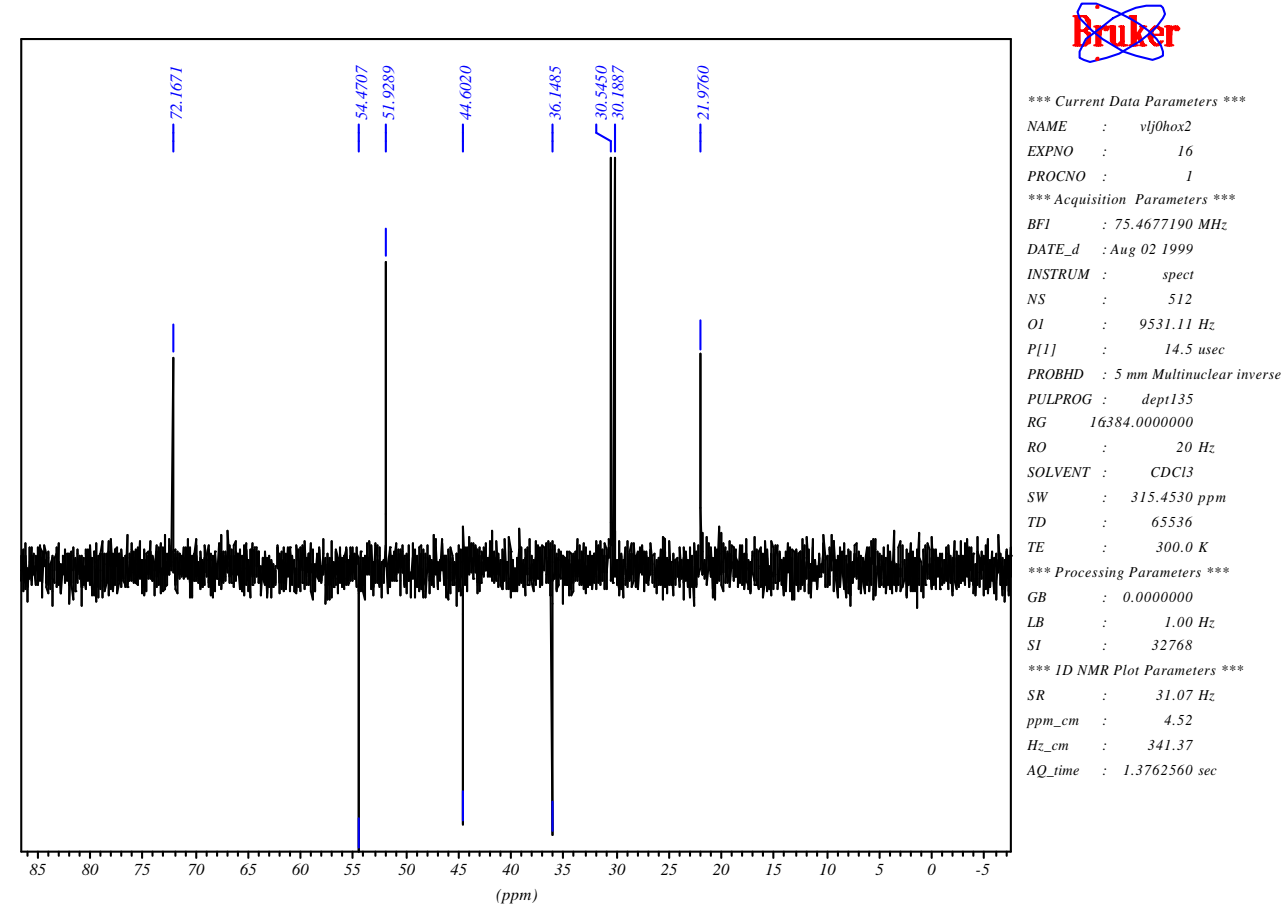


Espectro HMQC (2D H/C) do com posto (10b)

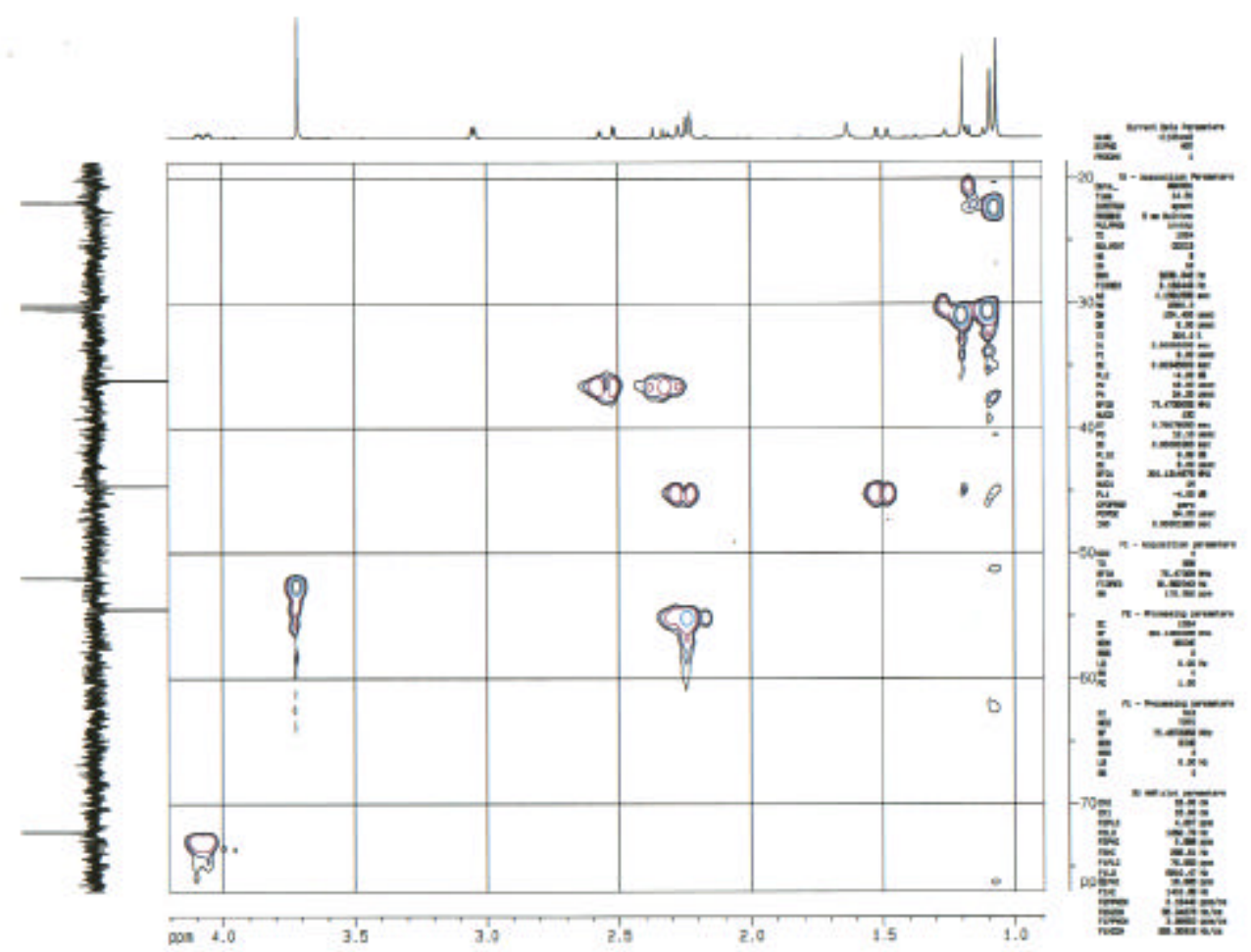

Espectros NOE DIFF do composto (10b)

$>$ irradiação na metila 6

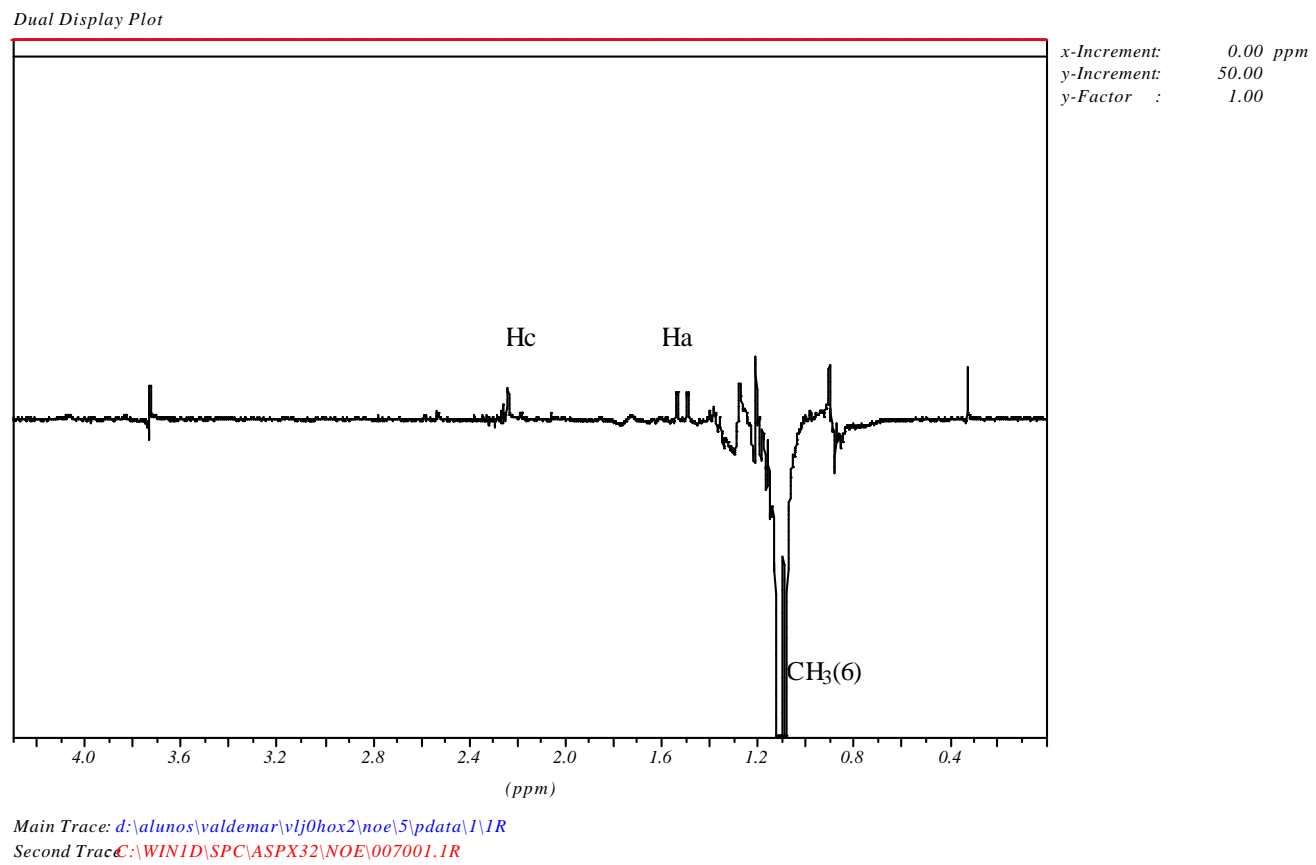


irradiação na metila 7

Dual Display Plot

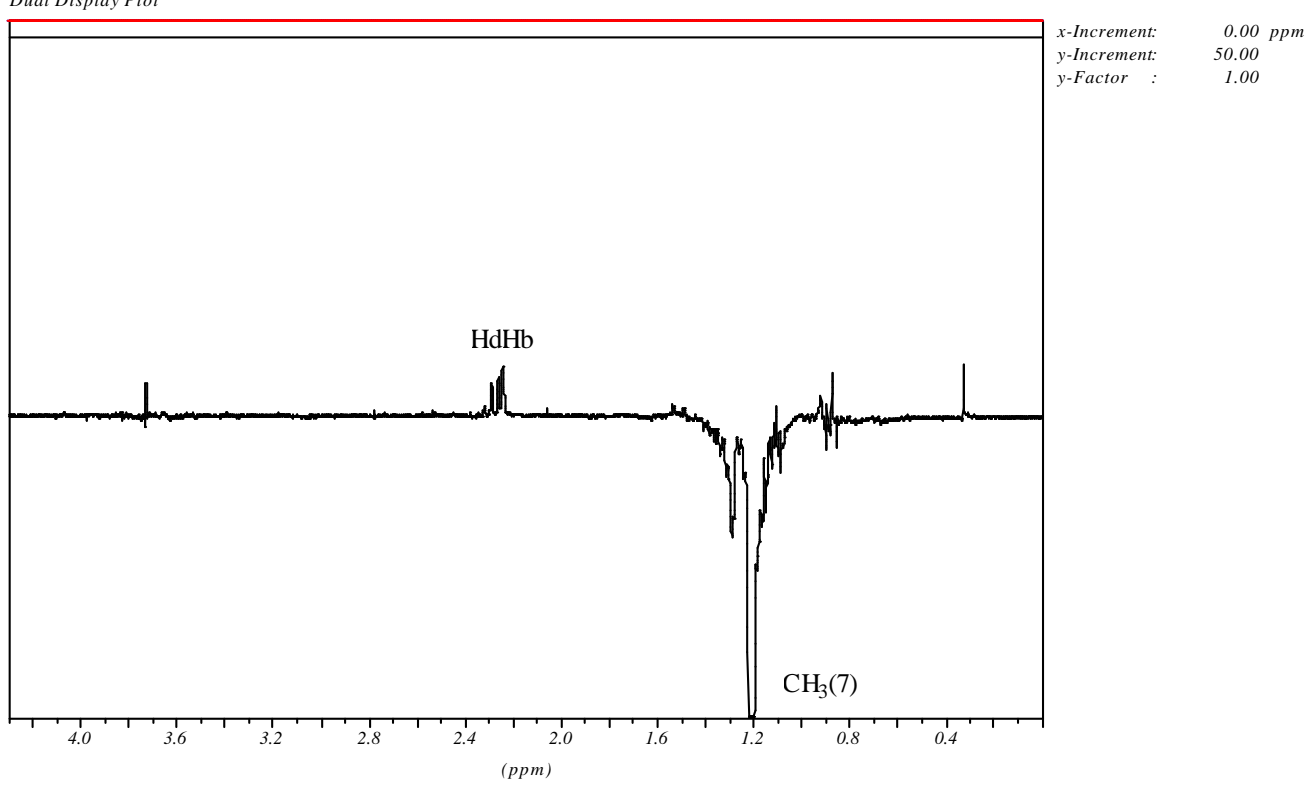

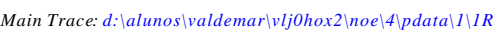

Second Trace: $\backslash$ WINID \SPC\ASPX32\NOE\007001.1R

irradiação na metila 8

Dual Display Plot

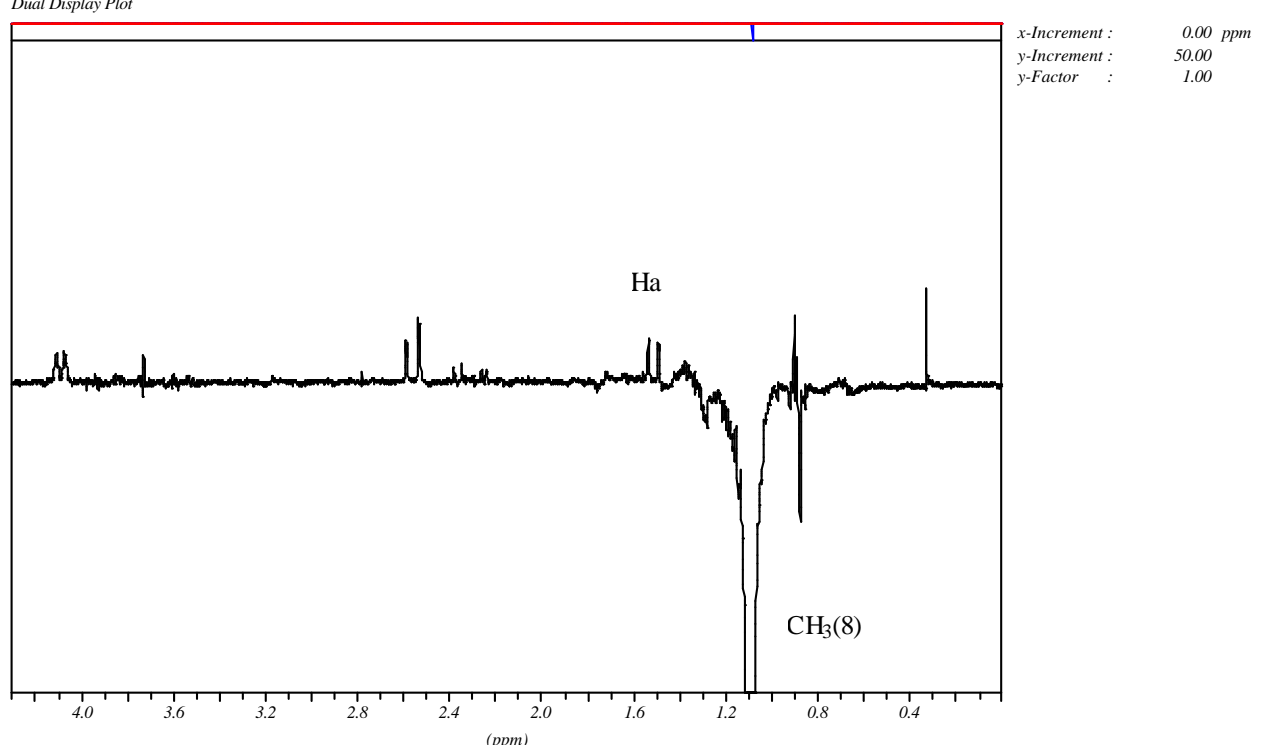

Main Trace : d:Valunossvaldemarvijohoxz VuoeloydatalVIR

Second Trace C:WWINIDISPCASPX32WOEV007001.IR 
Tabela 44 - Dados espectrais de NOE DIFF do composto $\mathbf{1 0 b}\left(\mathrm{CDCl}_{3}\right)$.

\begin{tabular}{c|c|c}
\hline$\delta(\mathbf{p p m})$ Irradiado & Atribuição & Efeito NOE Observado \\
& & $\mathrm{Hb}, \mathrm{Hd}$ \\
1,19 & $\mathrm{CH}_{3}(7)$ & $\mathrm{Ha}, \mathrm{Hc}$ \\
1,08 & $\mathrm{CH}_{3}(6)$ & $\mathrm{Ha}$ \\
\hline
\end{tabular}

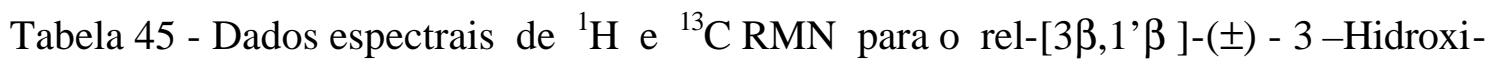
3-(1',4',4'-trimetil-2'-oxo-ciclopentil)-propanoato de metila $\left(\mathrm{CDCl}_{3}\right)$

\begin{tabular}{|c|c|c|}
\hline $\mathrm{C}-, \mathrm{H}_{-}$ & ${ }^{1} \mathrm{H}(300 \mathrm{MHz}) \delta(\mathrm{ppm})$ & $\begin{array}{c}{ }^{13} \mathrm{C} \\
(75 \mathrm{MHz})\end{array}$ \\
\hline 1 & & 222,36 \\
\hline 2 & $\begin{array}{c}\mathrm{Hd}-2,27(\mathrm{~d}, 1 \mathrm{H}, \mathrm{Jdc}=17,3 \mathrm{~Hz}) \text { e } \mathrm{Hc}-2,19(\mathrm{dd}, 1 \mathrm{H}, \mathrm{Jcd}=17,3 \mathrm{e} \\
\text { Jca }=1,4 \mathrm{~Hz})\end{array}$ & 54,47 \\
\hline 3 & & 32,93 \\
\hline 4 & $\begin{array}{c}\mathrm{Hb}-2,24(\mathrm{~d}, 1 \mathrm{H}, \mathrm{Jba}=13,2 \mathrm{~Hz}) \text { e Ha-1,5 (dd, } 1 \mathrm{H}, \mathrm{Jab}=13,2 \text { e Jac } \\
=1,4 \mathrm{~Hz})\end{array}$ & 44,60 \\
\hline 5 & & 53,16 \\
\hline 6 & $1,08(\mathrm{~s}, 3 \mathrm{H})$ & 30,18 \\
\hline 7 & $1,19(\mathrm{~s}, 3 \mathrm{H})$ & 30,54 \\
\hline 8 & $1,06(\mathrm{~s}, 3 \mathrm{H})$ & 21,97 \\
\hline 9 & $\begin{array}{c}\mathrm{He}-4,07(\mathrm{ddd}, 1 \mathrm{H}, \mathrm{Jef}=10,7 ; \mathrm{Je}-\mathrm{OH}=3,1 \text { e Jeg=2,3) e } 3,05(\mathrm{~d}, \\
1 \mathrm{H}, \mathrm{OH}, \text { Jон-e=3,1Hz) }\end{array}$ & 72,16 \\
\hline $10 *$ & $\begin{array}{c}\mathrm{Hg}-2,54(\mathrm{dd}, 1 \mathrm{H}, \mathrm{Jgf}=16,5 \text { e Jeg=2,3Hz) e Hf-2,32 (dd, 1H, } \\
\text { Jfg=16,5 e Jfe=10,7Hz) }\end{array}$ & 36,14 \\
\hline 11 & & 173,33 \\
\hline 12 & $3,71(\mathrm{~s}, 3 \mathrm{H})$ & 51,92 \\
\hline
\end{tabular}

* A atribuição dos sinais $\mathrm{Hg}$ e $\mathrm{Hf}$ foi feita baseado em estudos conformacionais realizados no programa PC Model 7.

Além dos compostos acima, também foram analisados os compostos 11, 12 ,13, preparados em nosso laboratório, ${ }^{26,27}$ e que possuem acoplamento a longa distância ${ }^{4} \mathrm{~J}_{\mathrm{HH}}$. 


\section{Composto (11)}

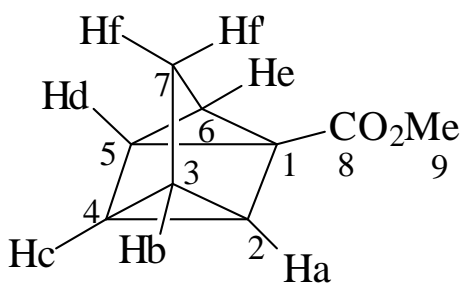

11

\section{Espectro de ${ }^{1} \mathrm{H}$-RMN do composto (11)}

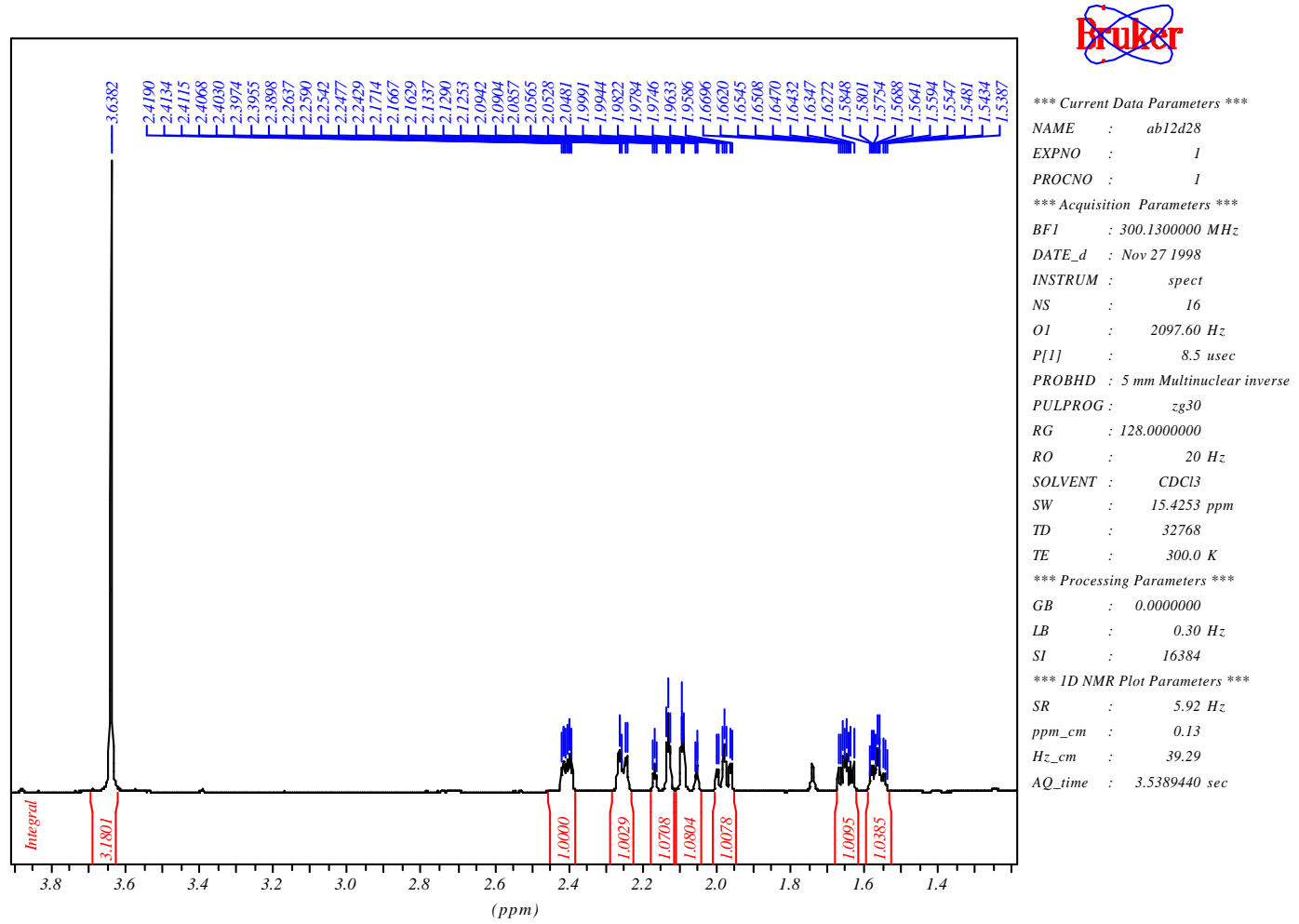

Tabela 46 - Dados espectrais de ${ }^{1} \mathrm{H}$ RMN do composto $11\left(\mathrm{CDCl}_{3}\right)$.

\begin{tabular}{c|c|c|c}
\hline$\delta(\mathbf{p p m})$ & Atribuição & Sinal & J (Hz) \\
\hline 2,4 & $\mathrm{Hd}$ & $\mathrm{ddd}$ & Jde $=4,9 ; \mathrm{Jdc}=2,3$ e Jda $=1,5 \mathrm{~Hz}$ \\
2,25 & $\mathrm{He}$ & $\mathrm{dt}$ & $\mathrm{Jed}=4,9 ; \mathrm{Jef}=\mathrm{Jef}^{\prime}=1,4 \mathrm{~Hz}$ \\
2,16 & $\mathrm{Hf}$ & $\mathrm{dt}$ & $\mathrm{Jff}{ }^{\prime}=11,3 ; \mathrm{Jfb}=\mathrm{Jfe}=1,4 \mathrm{~Hz}$ \\
2,13 & $\mathrm{Hf}^{\prime}$ & $\mathrm{dt}$ & $\mathrm{Jf} \mathrm{f}^{\prime}=11,3 ; \mathrm{Jf} f^{\prime} \mathrm{b}=\mathrm{Jf} \mathbf{e}^{\prime}=1,4 \mathrm{~Hz}$ \\
1,97 & $\mathrm{Ha}$ & $\mathrm{ddd}$ & $\mathrm{Jab}=6,3 ; \mathrm{Jac}=4,9 \mathrm{e} \mathrm{Jad}=1,5 \mathrm{~Hz}$ \\
1,65 & $\mathrm{Hc}$ & $\mathrm{ddd}$ & $\mathrm{Jcb}=4,9 ; \mathrm{Jca}=4,9$ e Jcd $=2,3 \mathrm{~Hz}$ \\
1,56 & $\mathrm{Hb}$ & $\mathrm{ddt}$ & $\mathrm{Jba}=6,3 ; \mathrm{Jbc}=4,9 ; \mathrm{Jbf}=\mathrm{Jbf}=1,4 \mathrm{~Hz}$ \\
3,65 & $9\left(\mathrm{OCH}_{3}\right)$ & $\mathrm{s}$ & \\
\hline
\end{tabular}




\section{Espectro de ${ }^{13}$ C-RMN (BB) do composto (11)}

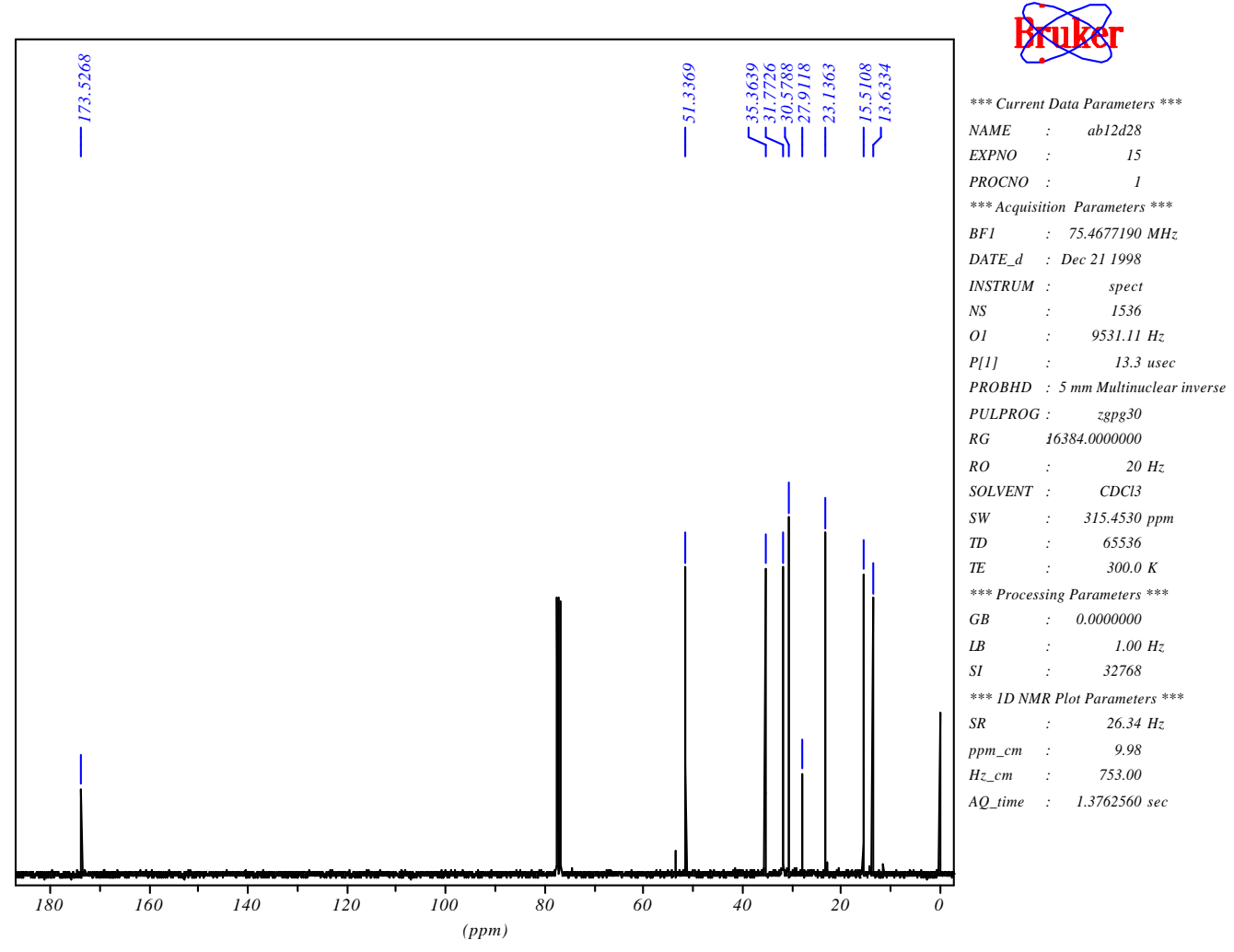

Espectro de ${ }^{13}$ C-RMN (DEPT-135) do composto (11)

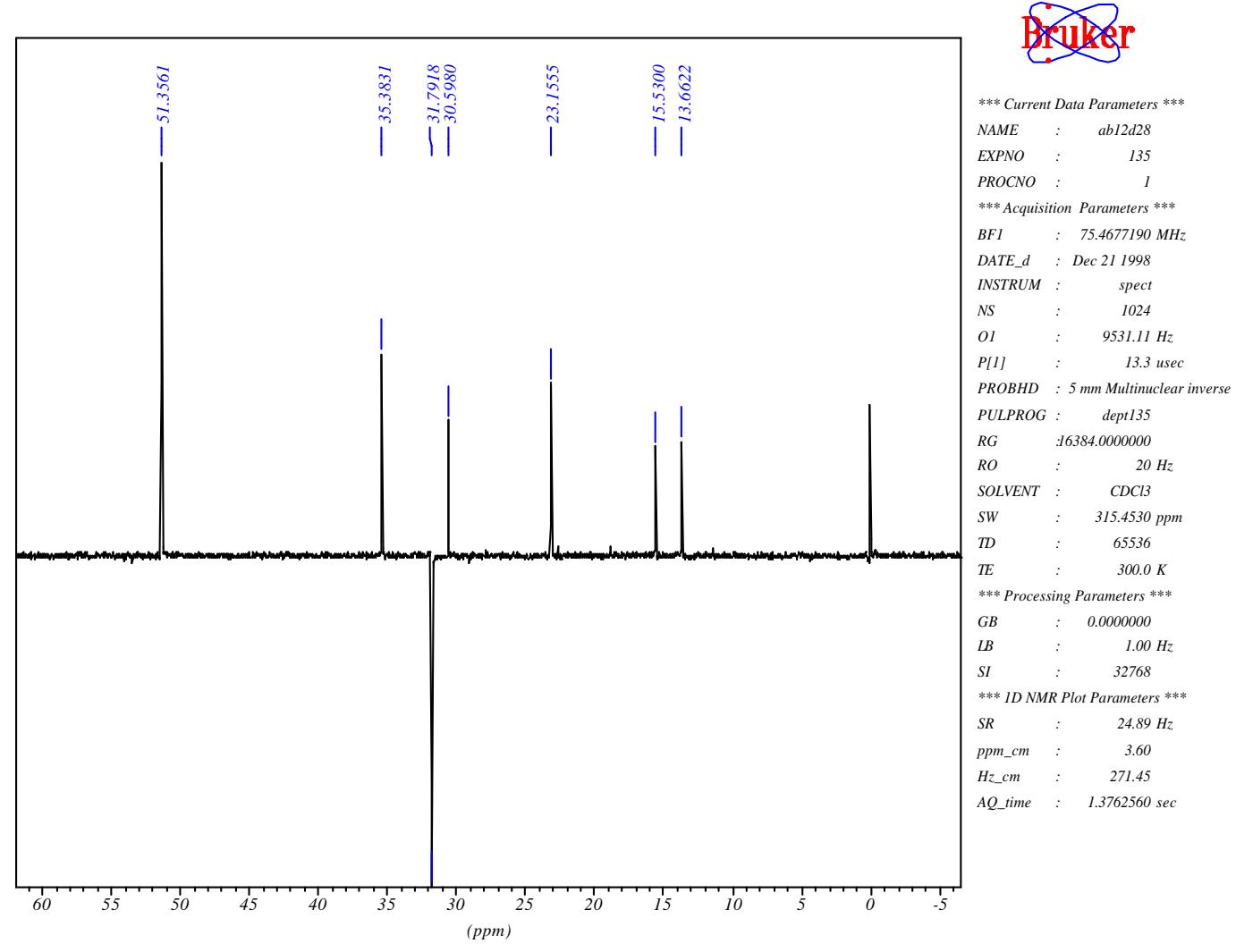


Tabela 47 - Dados espectrais de ${ }^{1} \mathrm{H}$ e ${ }^{13} \mathrm{C} \mathrm{RMN}$ para o Derivado de Quadriciclano $\left(\mathrm{CDCl}_{3}\right)$

\begin{tabular}{|c|c|c|}
\hline $\mathrm{C}-, \mathrm{H}-$ & ${ }^{1} \mathrm{H}(300 \mathrm{MHz}) \delta(\mathrm{ppm})$ & ${ }^{{ }^{13} \mathrm{C}}$ \\
\hline 1 & & 27,91 \\
\hline 2 & $1,97(\mathrm{ddd}, 1 \mathrm{H}, \mathrm{Jab}=6,3 ; \mathrm{Jac}=4,9$ e $\mathrm{Jad}=1,5 \mathrm{~Hz})$ & 30,57 \\
\hline 3 & $1,56\left(\mathrm{ddt}, 1 \mathrm{H}, \mathrm{Jba}=6,3 ; \mathrm{Jbc}=4,9 ; \mathrm{Jbf}=\mathrm{Jbf} \mathrm{f}^{\prime}=1,4 \mathrm{~Hz}\right)$ & 13,63 \\
\hline 4 & $1,65(\mathrm{ddd}, 1 \mathrm{H}, \mathrm{Jcb}=4,9 ; \mathrm{Jca}=4,9$ e Jcd $=2,3 \mathrm{~Hz})$ & 15,51 \\
\hline 5 & $2,4(\mathrm{ddd}, 1 \mathrm{H}, \mathrm{Jde}=4,9 ; \mathrm{Jdc}=2,3$ e Jda $=1,5 \mathrm{~Hz})$ & 35,36 \\
\hline 6 & $2,25\left(\mathrm{dt}, 1 \mathrm{H}, \mathrm{Jed}=4,9 ; \mathrm{Jef}=\mathrm{Jef}^{\prime}=1,4 \mathrm{~Hz}\right)$ & 23,13 \\
\hline 7 & $\begin{array}{c}\text { Hf-2,16(dt, 1H, Jff' }=11,3 ; \mathrm{Jfb}=\mathrm{Jfe}=1,4 \mathrm{~Hz}) \mathrm{Hf} f^{\prime}-2,13(\mathrm{dt}, 1 \mathrm{H}, \\
\left.\text { Jf'f }=11,3 ; \mathrm{Jf}{ }^{\prime} b=\mathrm{Jf}^{\prime} \mathrm{e}^{-}=1,4 \mathrm{~Hz}\right)\end{array}$ & 31,77 \\
\hline 8 & & 173,52 \\
\hline 9 & $3,65(\mathrm{~s}, 3 \mathrm{H})$ & 51,33 \\
\hline
\end{tabular}




\section{Composto (12)}

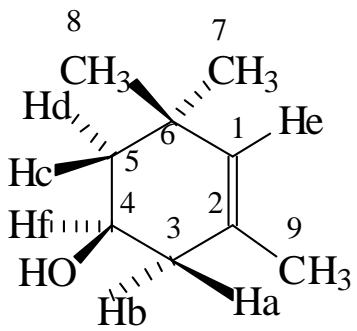

Espectro de ${ }^{1}$ H-RMN do composto (12)

12

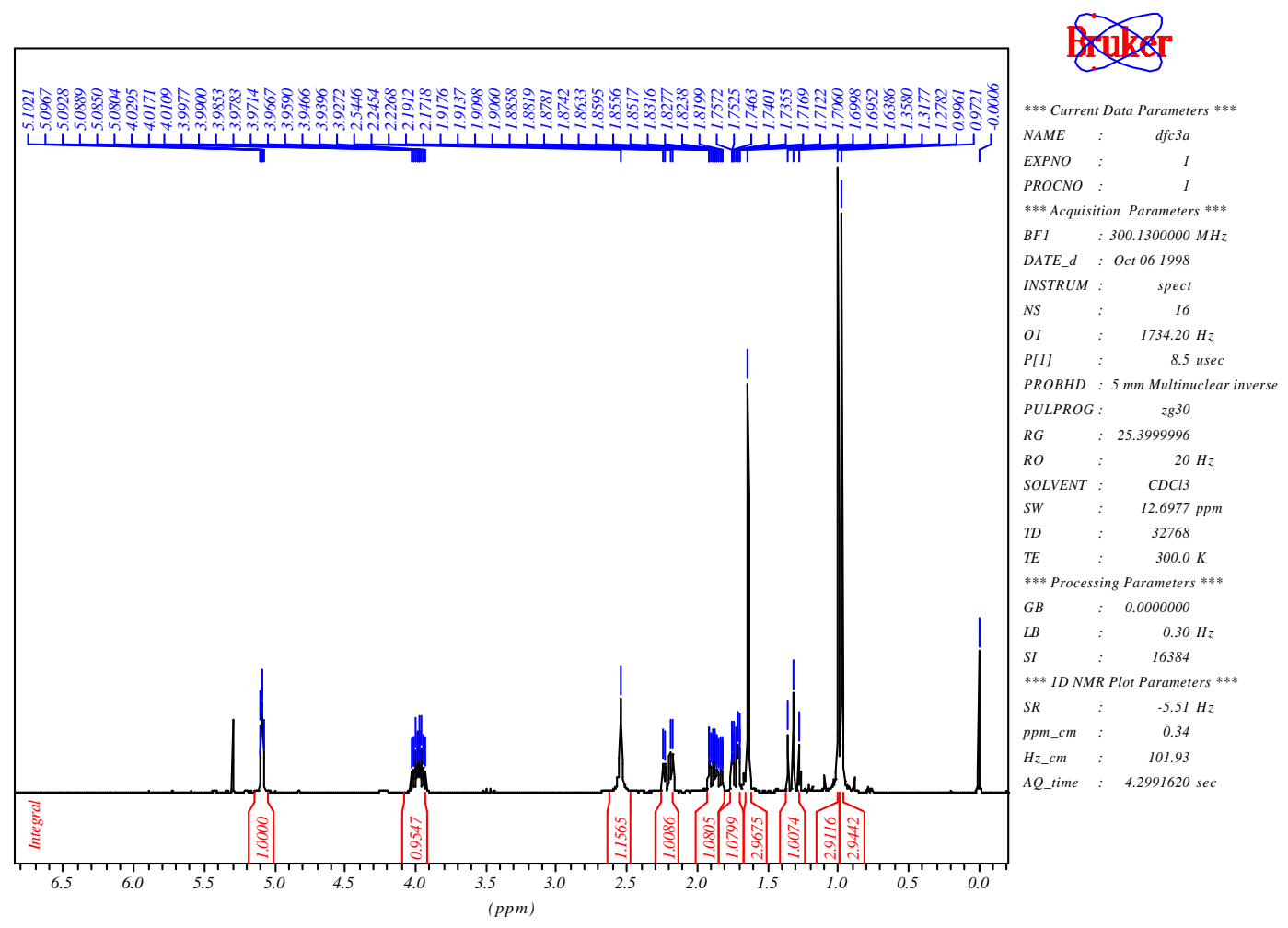

Tabela 48 - Dados espectrais de ${ }^{1} \mathrm{H}$ RMN do composto $12\left(\mathrm{CDCl}_{3}\right)$.

\begin{tabular}{l|c|c|c}
\hline$\delta(\mathbf{p p m})$ & Atribuição & Sinal & J $(\mathbf{H z})$ \\
\hline 5,1 & $\mathrm{He}$ & $\mathrm{dt}$ & $\mathrm{Jea}=2,4 \mathrm{e} \mathrm{Jeb}=\mathrm{Jed}=1,3 \mathrm{~Hz}$ \\
3,97 & $\mathrm{Hf}$ & $\mathrm{dddd}$ & $\mathrm{Jfc}=12,1 ; \mathrm{Jfa}=9,5 ; \mathrm{Jfb}=5,7 ;$ \\
& $\mathrm{Jfd}=3,7 \mathrm{~Hz}$ \\
2,54 & $\mathrm{OH}$ & $\mathrm{sl}$ & \\
2,20 & $\mathrm{Hb}$ & $\mathrm{ddt}$ & $\mathrm{Jba}=16,2 ; \mathrm{Jbf}=5,7 ; \mathrm{Jbe}=\mathrm{Jbd}=1,3$ \\
& & & $\mathrm{~Hz}$ \\
1,86 & $\mathrm{Ha}$ & $\mathrm{ddd}$ & $\mathrm{Jab}=16,2 ; \mathrm{Jaf}=9,5 \mathrm{e} \mathrm{Jae}=2,4 \mathrm{~Hz}$ \\
1,72 & $\mathrm{Hd}$ & $\mathrm{ddt}$ & $\mathrm{Jdc}=12 ; \mathrm{Jdf}=3,7 \mathrm{e} \mathrm{Jde}=\mathrm{Jdb}=1,3 \mathrm{~Hz}$ \\
1,63 & $\mathrm{CH}_{3}(9)$ & $\mathrm{s}$ & $\mathrm{Jcd}=\mathrm{Jcf}=12 \mathrm{~Hz}$ \\
1,31 & $\mathrm{Hc}$ & $\mathrm{t}$ & \\
0,99 & $\mathrm{CH}_{3}(8)$ & $\mathrm{s}$ & $\mathrm{CH}_{3}(7)$
\end{tabular}




\section{Espectro de ${ }^{13}$ C-RMN (BB) do composto (12)}

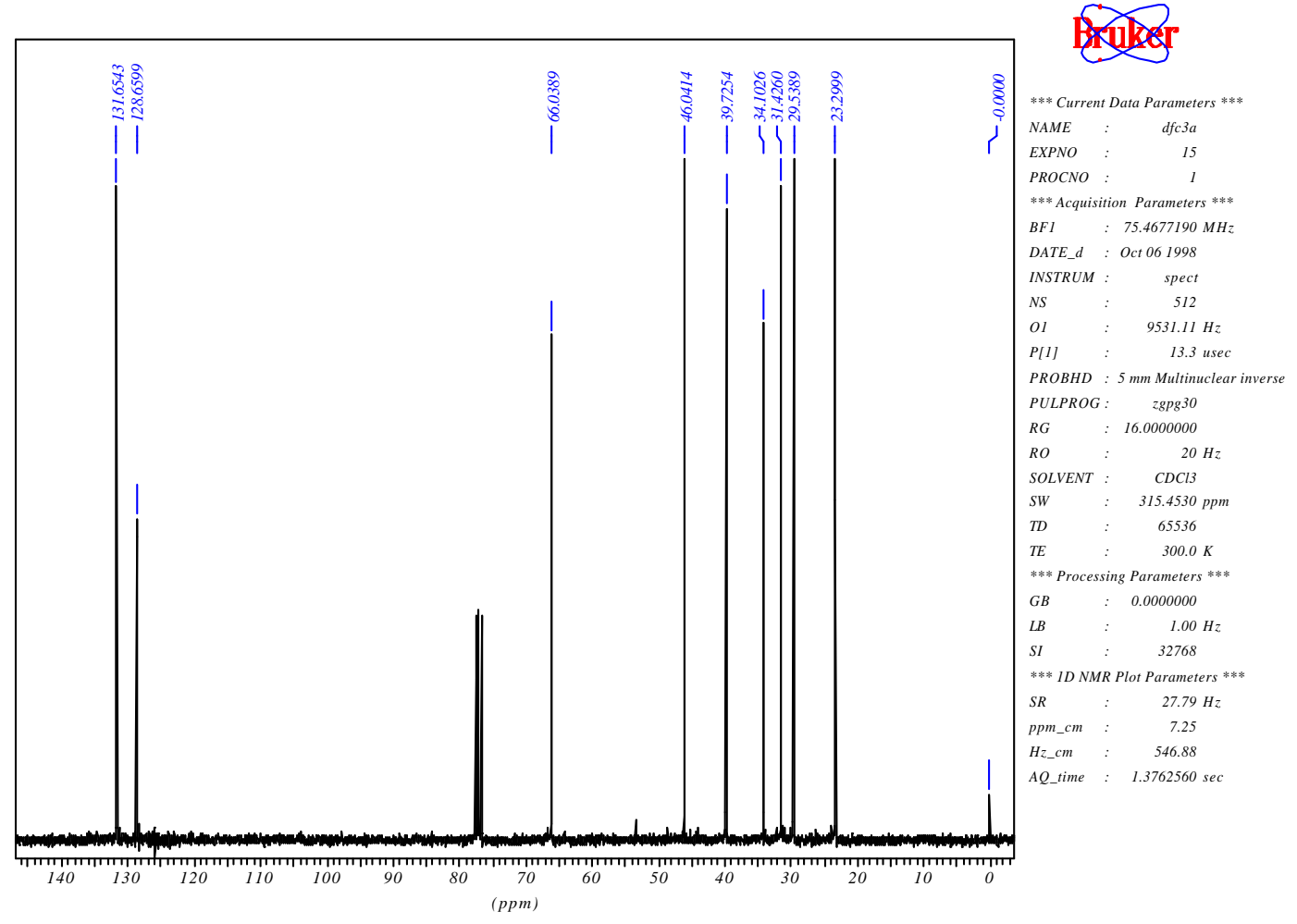

Espectro de ${ }^{13}$ C-RMN (DEPT-135) do composto (12)

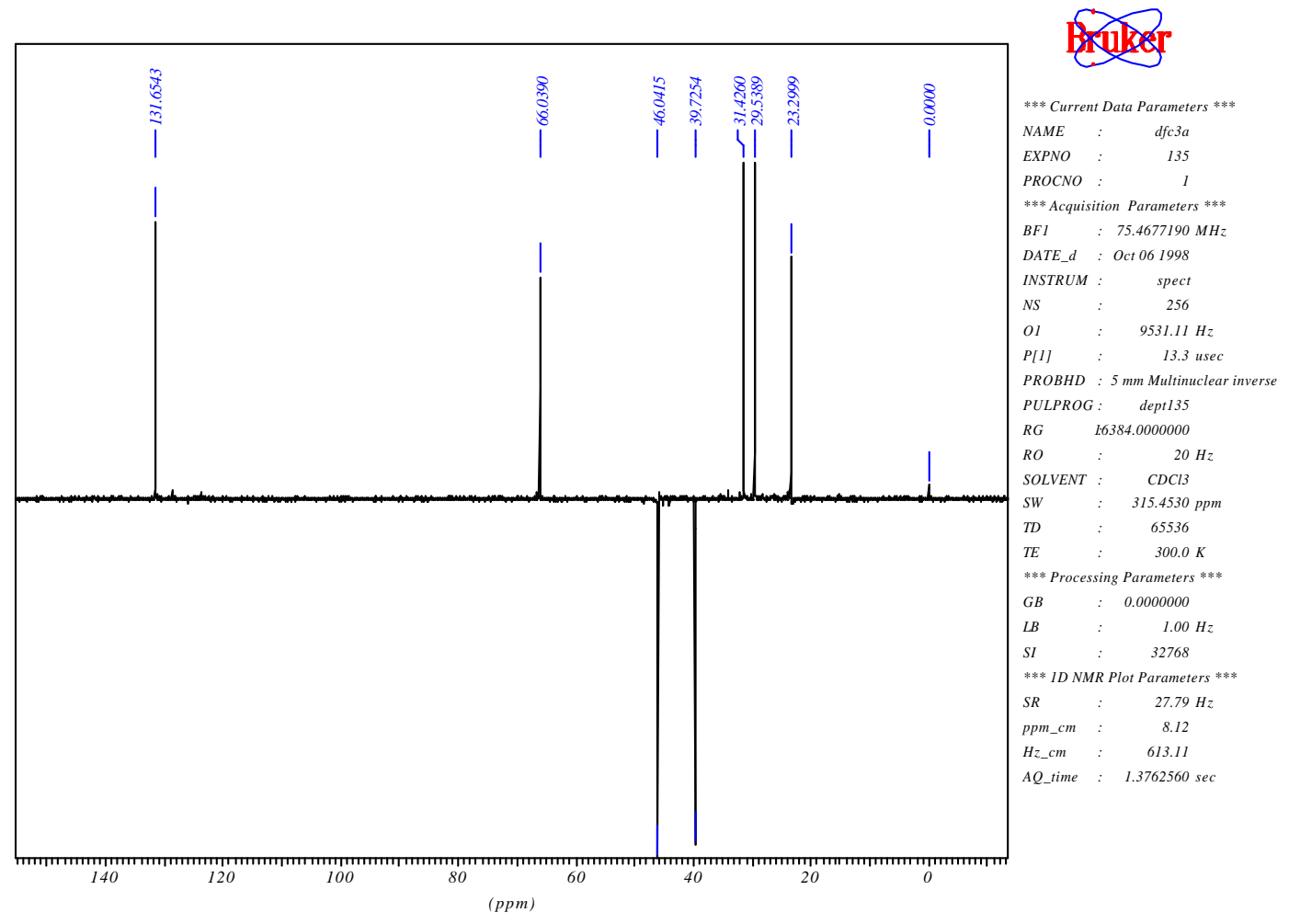


Espectro HMQC (2D H/C) do composto (12)

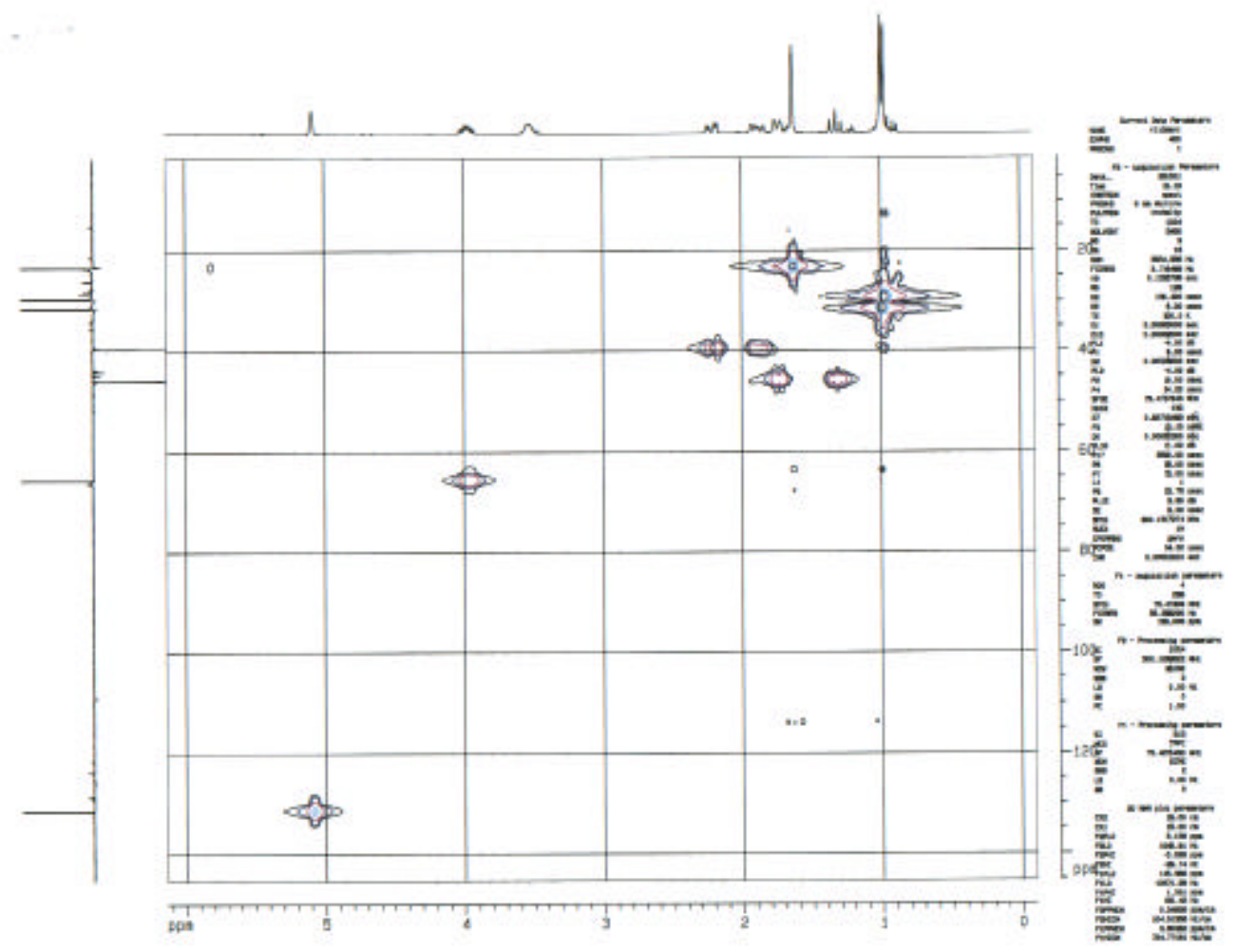

\section{Espectros NOE DIFF do composto (12)}

$>$ irradiação na metila 7

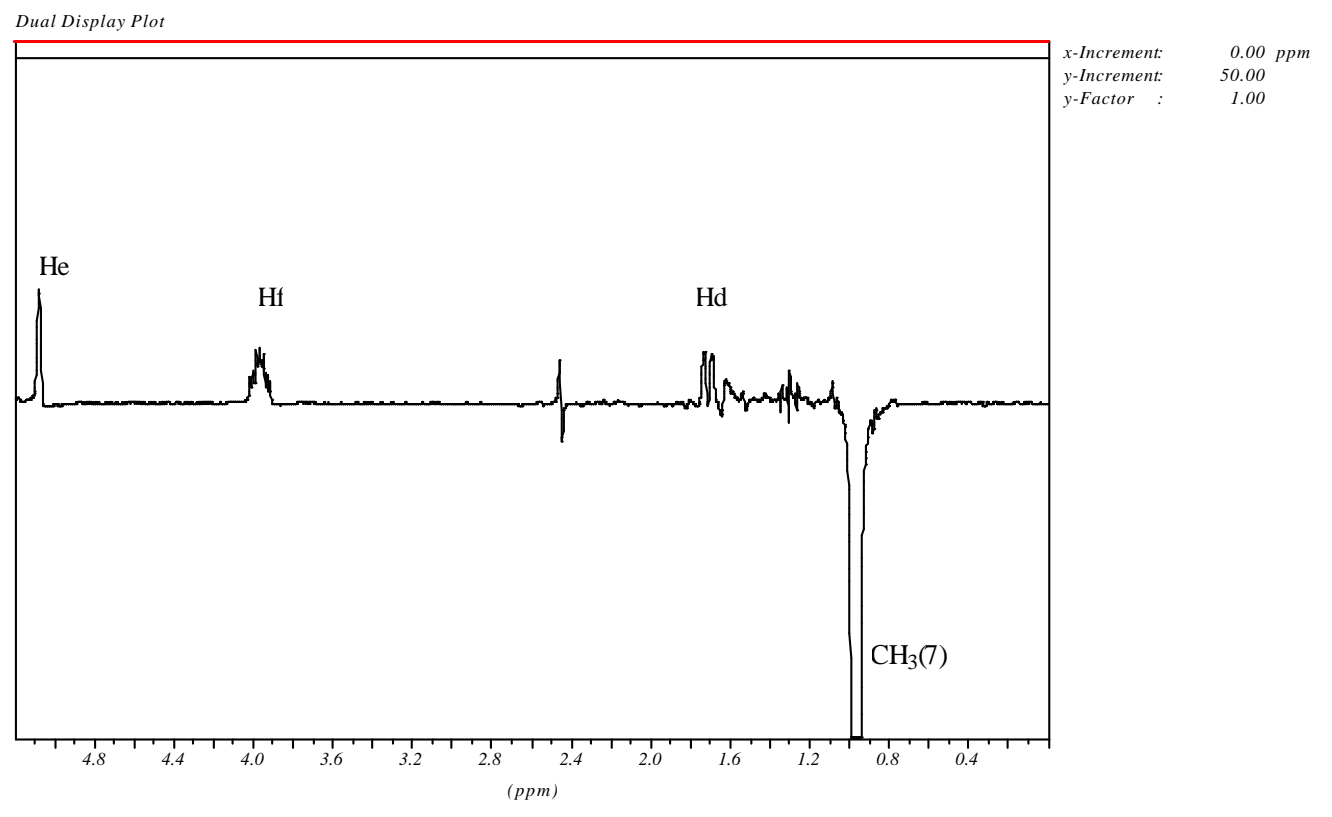

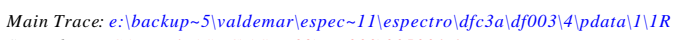
Second Trace: $\backslash$ WINID $\backslash S P C \backslash A S P X 32 \backslash D F 003 \backslash 005001.1 R$ 
irradiação na metila 8

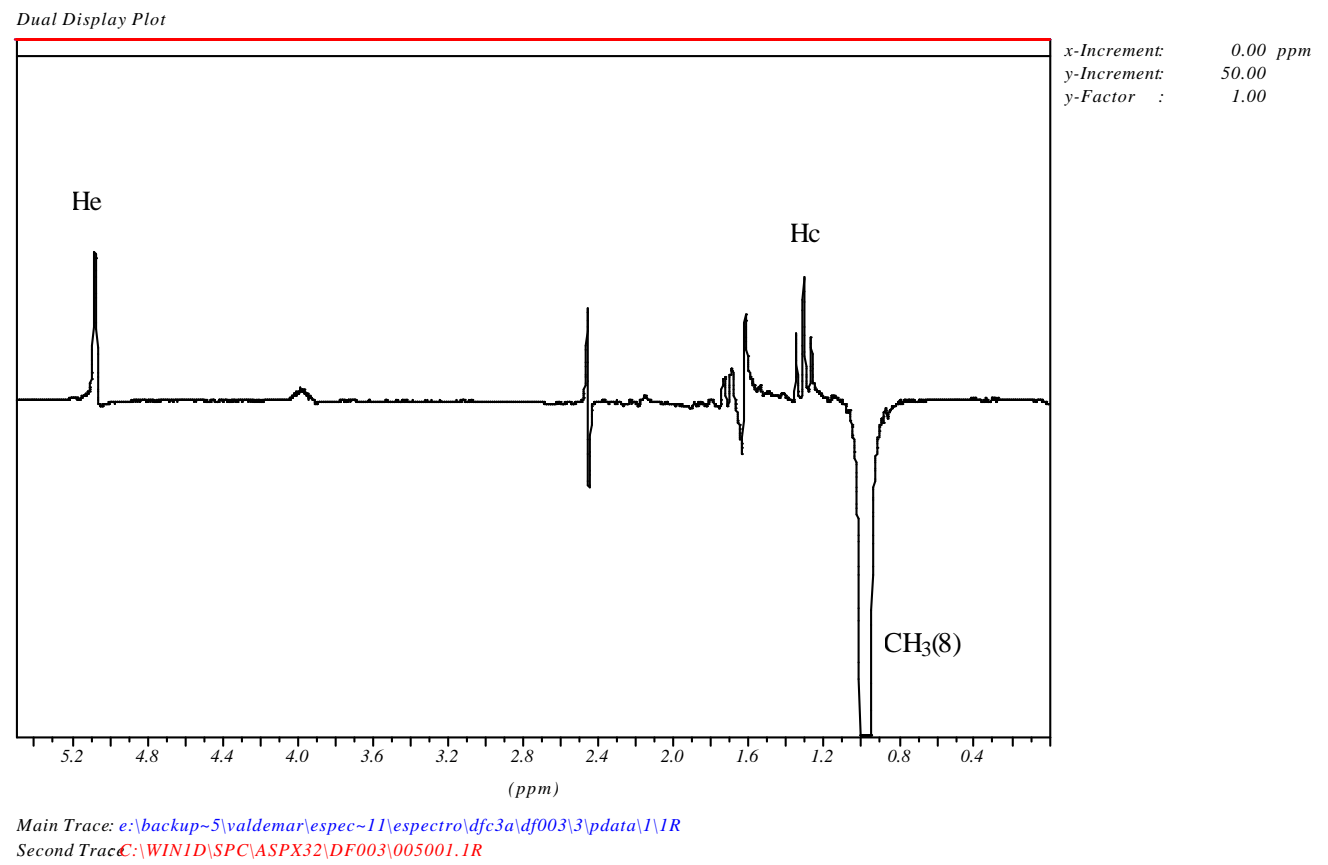

Tabela 49 - Dados espectrais de NOE DIFF do composto $12\left(\mathrm{CDCl}_{3}\right)$.

\begin{tabular}{c|c|c}
\hline$\delta$ (ppm)Irradiado & Atribuição & Efeito NOE Observado \\
1,63 & $\mathrm{CH}_{3}(9)$ & $\mathrm{Ha}, \mathrm{He}$ \\
0,99 & $\mathrm{CH}_{3}(8)$ & $\mathrm{Hc}, \mathrm{He}$ \\
0,97 & $\mathrm{CH}_{3}(7)$ & $\mathrm{Hd}, \mathrm{He}, \mathrm{Hf}$ \\
\hline
\end{tabular}

Tabela 50 - Dados espectrais de ${ }^{1} \mathrm{H}$ e ${ }^{13} \mathrm{C}$ RMN para o $( \pm)$ - 3,5,5-trimetil-ciclohex3-en-1-ol $\left(\mathrm{CDCl}_{3}\right)$

\begin{tabular}{|c|c|c|}
\hline $\mathrm{C}-, \mathrm{H}-$ & ${ }^{1} \mathrm{H}(300 \mathrm{MHz}) \delta(\mathrm{ppm})$ & ${ }^{13} \mathrm{C}(75 \mathrm{MHz})$ \\
\hline 1 & $\mathrm{He}-5,1(\mathrm{dt}, 1 \mathrm{H}, \mathrm{Jea}=2,4 \mathrm{e} \mathrm{Jeb}=\mathrm{Jed}=1,3 \mathrm{~Hz})$ & 131,65 \\
\hline 2 & & 128,65 \\
\hline 3 & $\begin{array}{c}\mathrm{Hb}-2,20(\mathrm{ddt}, 1 \mathrm{H}, \mathrm{Jba}=16,5 ; \mathrm{Jbf}=5,7 ; \mathrm{Jbe}=\mathrm{Jbd}=1,3 \mathrm{~Hz}) \text { e Ha- } \\
1,86(\mathrm{ddd}, 1 \mathrm{H}, \mathrm{Jab}=16,2 ; \mathrm{Jaf}=9,5 \mathrm{e} \mathrm{Jae}=2,4 \mathrm{~Hz})\end{array}$ & 39,72 \\
\hline 4 & $\begin{array}{c}\mathrm{Hf}-3,97(\mathrm{dddd}, 1 \mathrm{H}, \mathrm{Jfc}=12,1 ; \mathrm{Jfa}=9,5 ; \mathrm{Jfb}=5,7 ; \mathrm{Jfd}=3,7 \mathrm{~Hz}) \mathrm{e} \\
2,54(\mathrm{sl}, 1 \mathrm{H}, \mathrm{OH})\end{array}$ & 66,03 \\
\hline 5 & $\begin{array}{c}\mathrm{Hd}-1,72(\mathrm{ddt}, 1 \mathrm{H}, \mathrm{Jdc}=12 ; \mathrm{Jdf}=3,7 \text { e Jde=Jdb=1,3 Hz) e Hc- } \\
1,31(\mathrm{t}, 1 \mathrm{H}, \mathrm{Jcd}=\mathrm{Jcf}=12 \mathrm{~Hz})\end{array}$ & 46,04 \\
\hline 6 & $0,97(\mathrm{~s}, 3 \mathrm{H})$ & 34,10 \\
\hline 7 & $0,99(\mathrm{~s}, 3 \mathrm{H})$ & 29,53 \\
\hline 8 & $1,63(\mathrm{~s}, 3 \mathrm{H})$ & 31,42 \\
\hline 9 & & 23,29 \\
\hline
\end{tabular}




\section{Composto (13)}

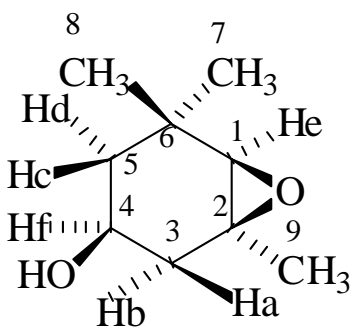

13

Espectro de ${ }^{1} \mathrm{H}-\mathrm{RMN}$ do composto (13)

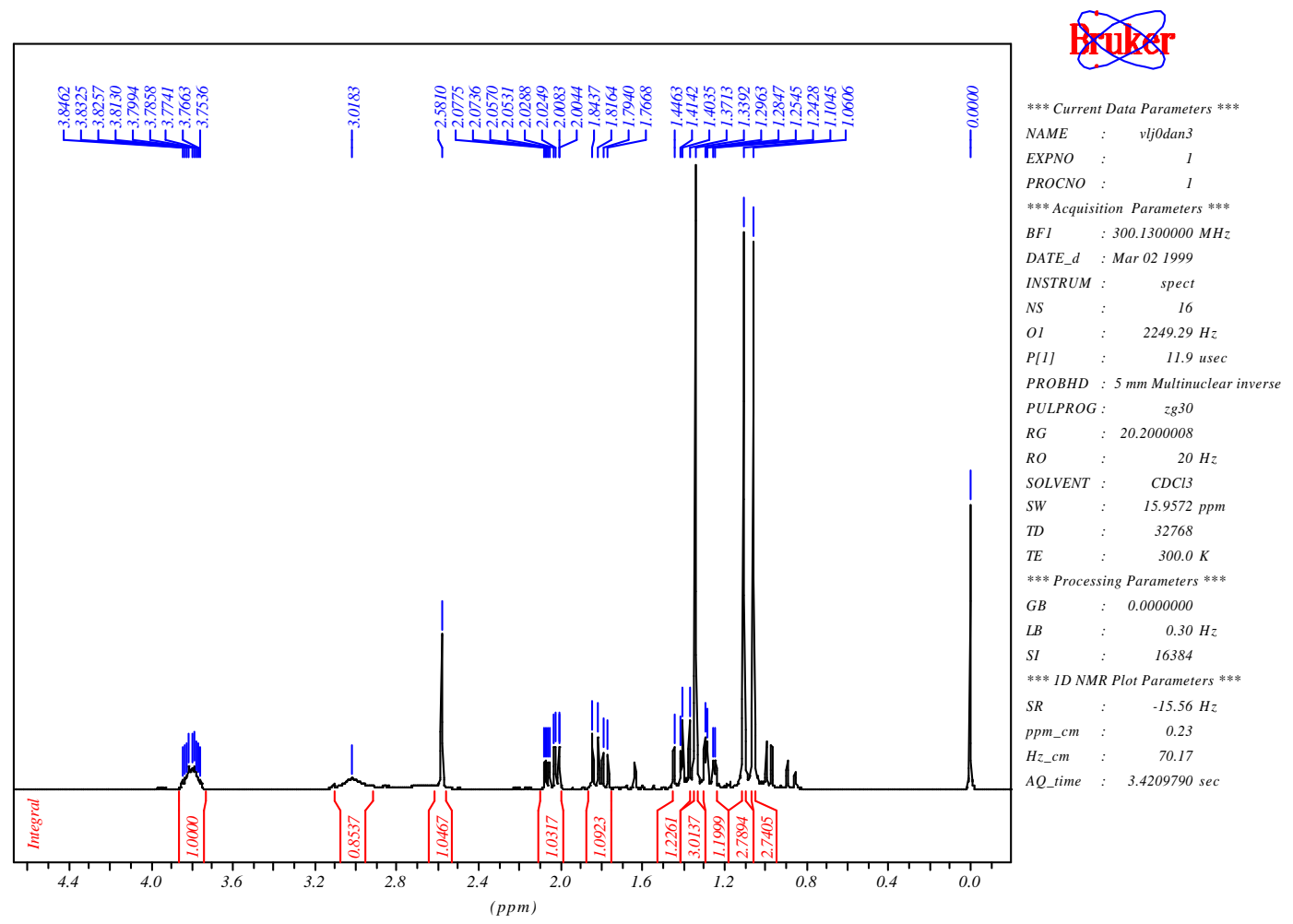

Tabela 51 - Dados espectrais de ${ }^{1} \mathrm{H}$ RMN do composto $13\left(\mathrm{CDCl}_{3}\right)$.

\begin{tabular}{|c|c|c|c|}
\hline$\delta(\mathbf{p p m})$ & Atribuição & Sinal & $\mathbf{J}(\mathbf{H z})$ \\
\hline 3,81 & Hf & dddd & $\begin{array}{c}\mathrm{Jfc}=9,7 ; \mathrm{Jfa}=8,2 ; \mathrm{Jfb}=6,1 \mathrm{e} \\
\mathrm{Jfd}=3,8 \mathrm{~Hz}\end{array}$ \\
\hline 3,02 & $\mathrm{OH}$ & sl & \\
\hline 2,6 & $\mathrm{He}$ & $\mathrm{s}$ & \\
\hline 1,83 & $\mathrm{Hb}$ & ddd & $\mathrm{Jba}=14,7 ; \mathrm{Jbf}=6,1$ e $\mathrm{Jbd}=1,4 \mathrm{~Hz}$ \\
\hline 1,80 & $\mathrm{Ha}$ & ddd & $\mathrm{Jab}=14,7 ; \mathrm{Jaf}=8,2$ e $\mathrm{Jad}=0,9 \mathrm{~Hz}$ \\
\hline 1,4 & $\mathrm{Hc}$ & dd & $\mathrm{Jcd}=12,8$ e Jcf $=9,6 \mathrm{~Hz}$ \\
\hline 1,34 & $\mathrm{CH}_{3}(9)$ & $\mathrm{s}$ & \\
\hline 1,26 & $\mathrm{Hd}$ & dddd & $\begin{array}{c}\mathrm{Jdc}=12,8 ; \mathrm{Jdf}=3,8 ; \mathrm{Jdb}=1,4 \mathrm{e} \\
\mathrm{Jda}=0,9 \mathrm{~Hz}\end{array}$ \\
\hline 1,10 & $\mathrm{CH}_{3}(8)$ & $\mathrm{S}$ & \\
\hline 1,06 & $\mathrm{CH}_{3}(7)$ & $\mathrm{s}$ & \\
\hline
\end{tabular}




\section{Espectro de ${ }^{13}$ C-RMN (BB) do composto (13)}

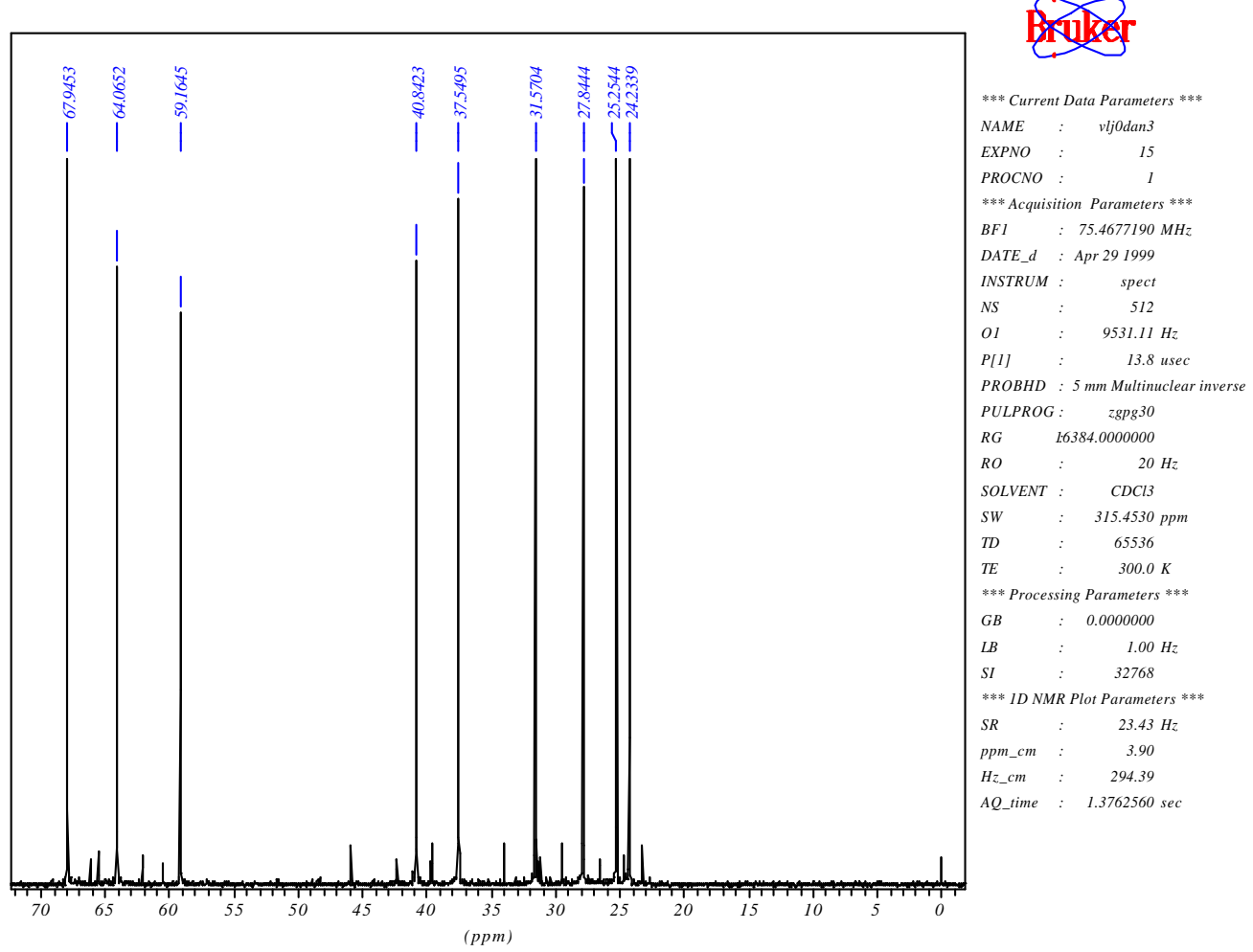

\section{Espectro de ${ }^{13}$ C-RMN (DEPT-135) do composto (13)}

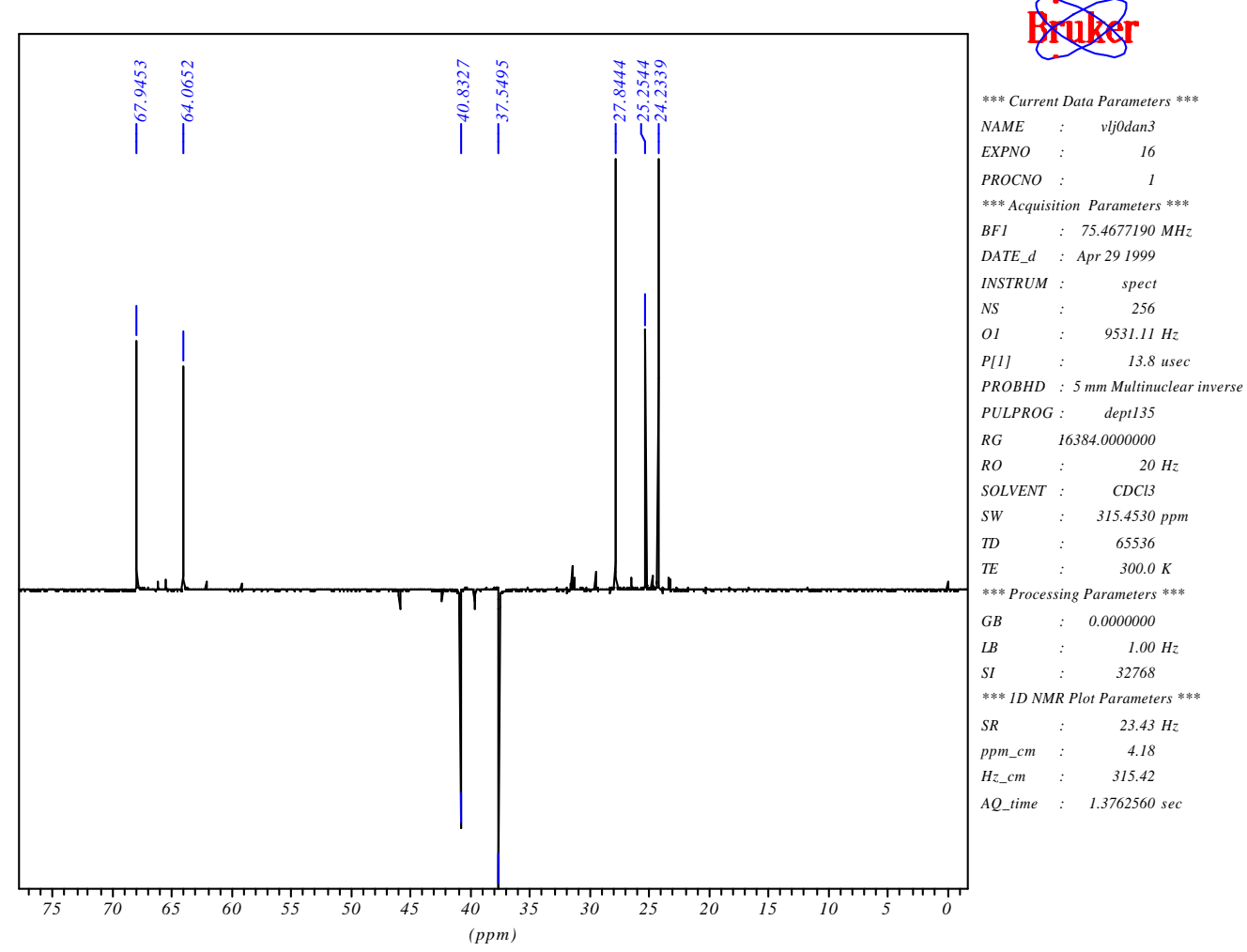




\section{Espectro HMQC (2D H/C) do composto (13)}

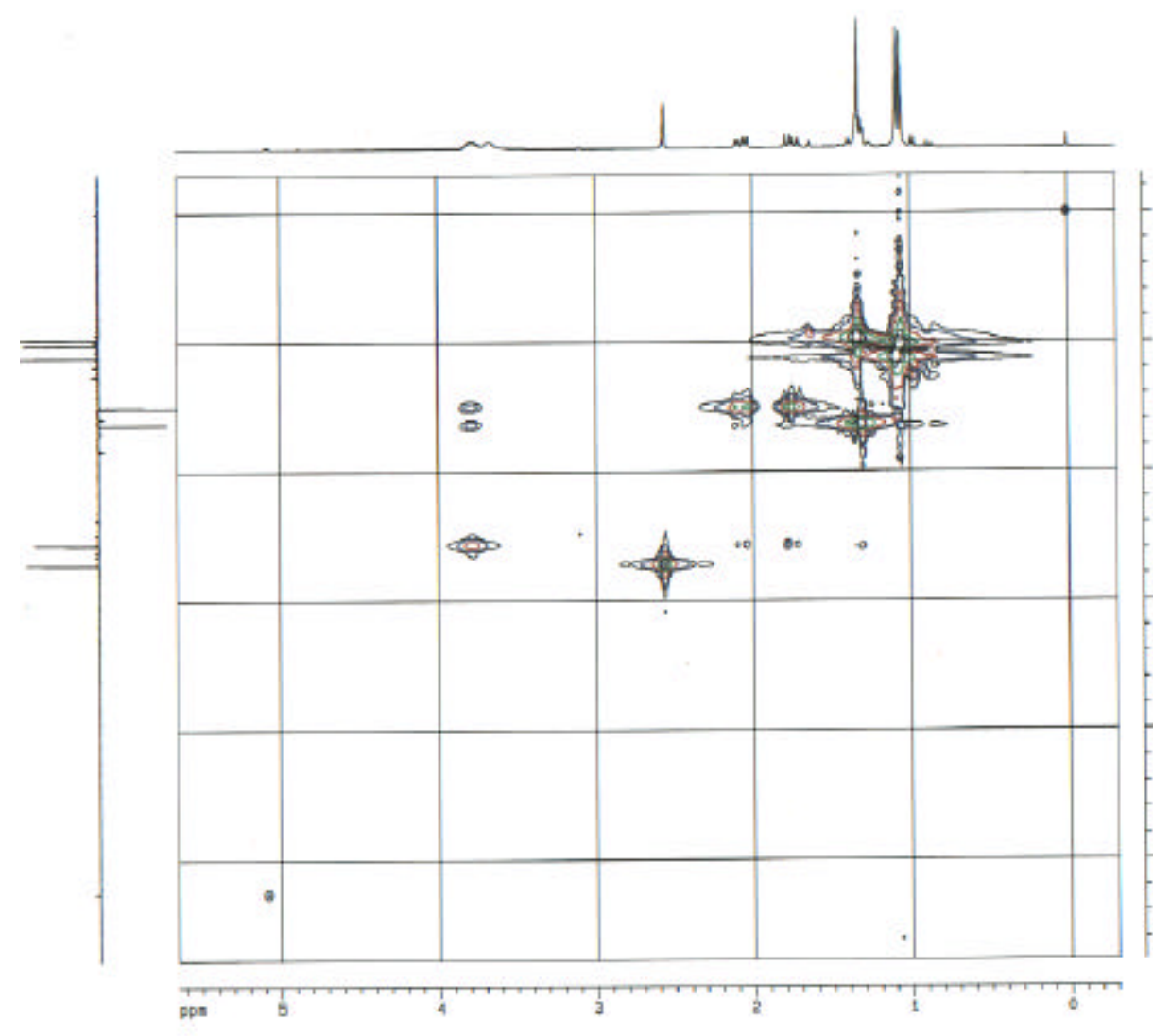

\section{Espectros NOE DIFF do composto (13)}

irradiação na metila 7

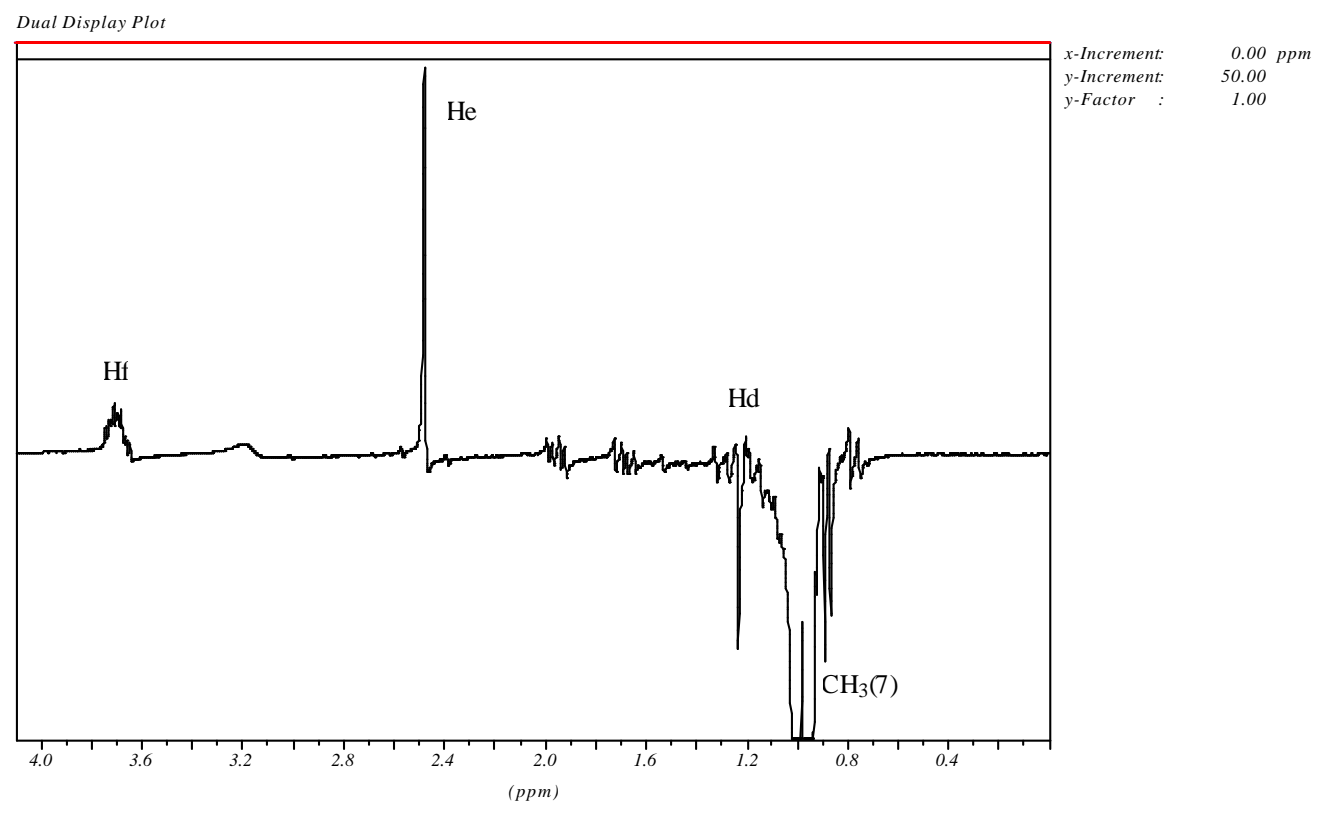

Main Trace: $d: \backslash a l u n o s \backslash v a l d e m a r \backslash v l j 0 d a n 3 \backslash$ noe $\backslash 705 \backslash p d a t a \backslash \backslash \backslash I R$ 
irradiação na metila 8

Dual Display Plot

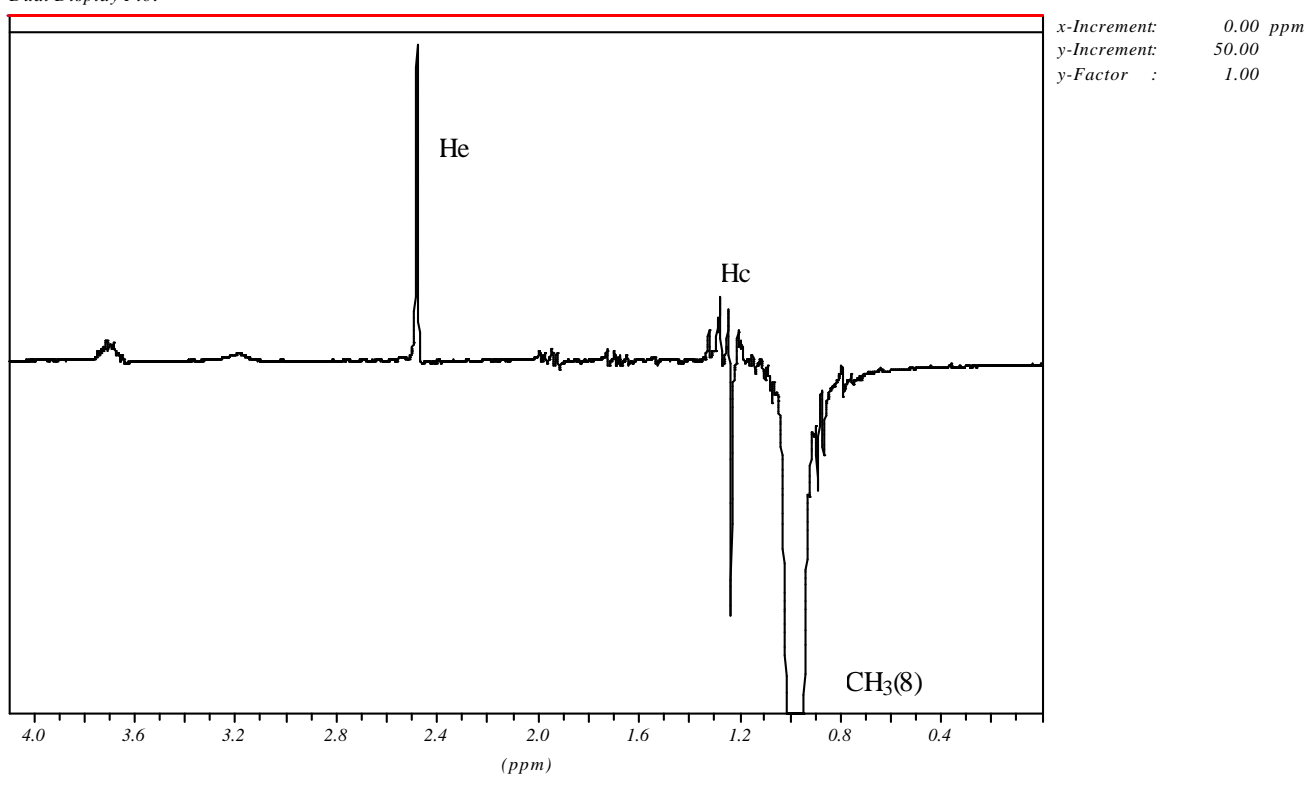

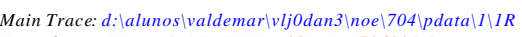

Second Trace: $\backslash W I N I D \backslash S P C \backslash A S P X 32 \backslash N O E \backslash 706001.1 R$

irradiação na metila 9

Dual Display Plot

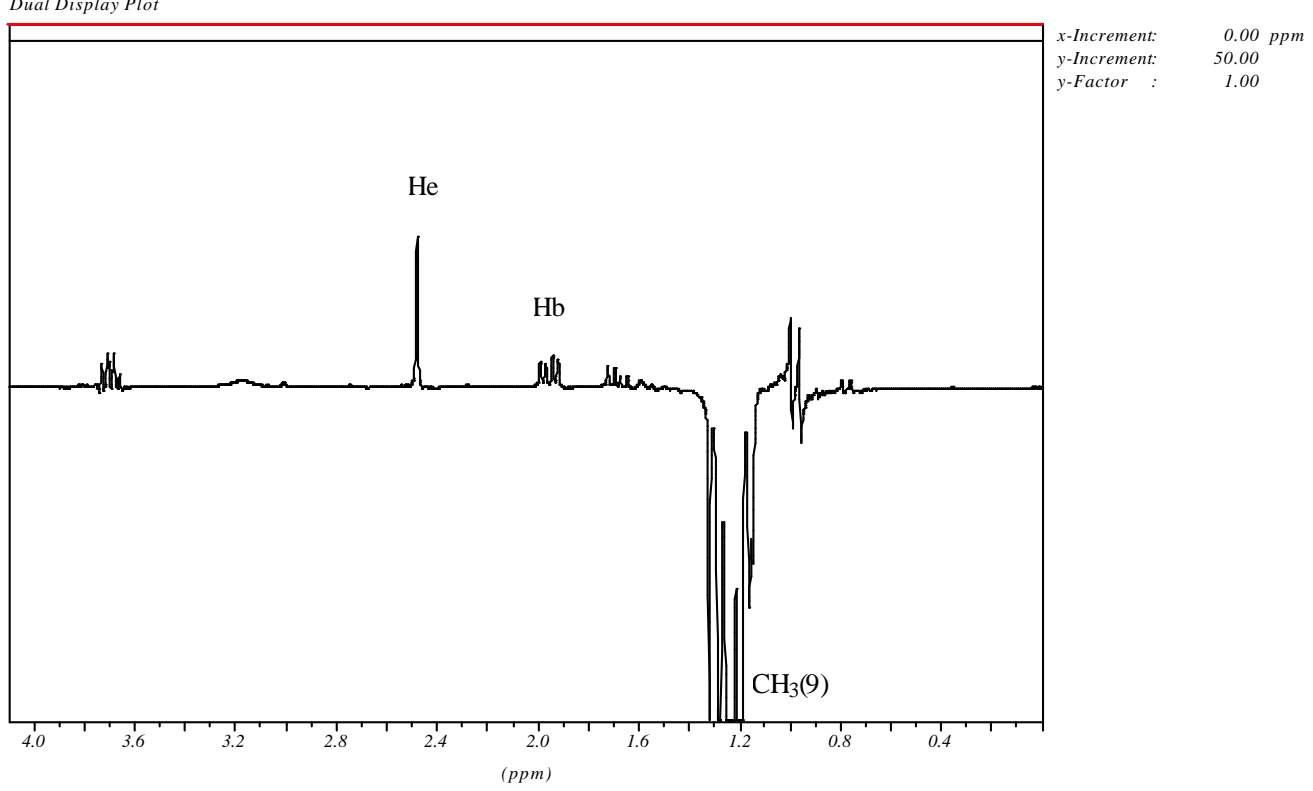

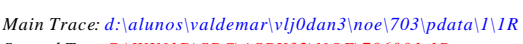

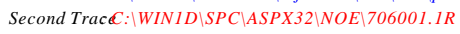


Tabela 52 - Dados espectrais de NOE DIFF do composto $13\left(\mathrm{CDCl}_{3}\right)$.

\begin{tabular}{c|c|c}
\hline$\delta(\mathbf{p p m})$ Irradiado & Atribuição & Efeito NOE Observado \\
\hline 1,34 & $\mathrm{CH}_{3}(9)$ & $\mathrm{Hb}, \mathrm{He}$ \\
1,10 & $\mathrm{CH}_{3}(8)$ & $\mathrm{Hc}, \mathrm{He}$ \\
1.06 & $\mathrm{CH}_{3}(7)$ & $\mathrm{Hd}, \mathrm{He}, \mathrm{Hf}$ \\
\hline
\end{tabular}

Tabela 53 - Dados espectrais de ${ }^{1} \mathrm{H}$ e ${ }^{13} \mathrm{C} \mathrm{RMN}$ para o $( \pm)$ - cis-1,5,5-trimetil-7oxabiciclo[4.1.0]heptan-3-ol $\left(\mathrm{CDCl}_{3}\right)$

\begin{tabular}{|c|c|c|}
\hline $\mathrm{C}-, \mathrm{H}-$ & ${ }^{1} \mathrm{H}(300 \mathrm{MHz}) \delta(\mathrm{ppm})$ & ${ }^{3} \mathrm{C}(75 \mathrm{MHz})$ \\
\hline 1 & $\mathrm{He}-2,6(\mathrm{~s}, 1 \mathrm{H})$ & 67,94 \\
\hline 2 & & 59,16 \\
\hline 3 & $\begin{array}{c}\mathrm{Hb}-1,83(\mathrm{ddd}, 1 \mathrm{H}, \mathrm{Jba}=14,7 ; \mathrm{Jbf}=6,1 \text { e Jbd=1,4 Hz) e Ha-1,80 } \\
\text { (ddd, } 1 \mathrm{H}, \mathrm{Jab}=14,7 ; \mathrm{Jaf}=8,2 \text { e Jad=0,9 Hz) }\end{array}$ & 37,84 \\
\hline 4 & $\mathrm{Hf}-3,8(\mathrm{dddd}, 1 \mathrm{H}, \mathrm{Jfc}=9,7 ; \mathrm{Jfa}=8,2 ; \mathrm{Jfb}=6,1$ e Jfd=3,8 Hz) e \\
& $3,02(\mathrm{sl}, 1 \mathrm{H}, \mathrm{OH})$ & 64,06 \\
\hline 5 & $\begin{array}{c}\mathrm{Hc}-1,4(\mathrm{dd}, 1 \mathrm{H}, \mathrm{Jcd}=12,8 \text { e Jcf=9,6 Hz) e Hd-1,26 (dddd, } 1 \mathrm{H}, \\
\text { Jdc=12,8; Jdf=3,8; Jdb=1,4 e Jda=0,9 Hz) }\end{array}$ & 40,84 \\
\hline 6 & & 31,57 \\
\hline 7 & $1,06(\mathrm{~s}, 3 \mathrm{H})$ & 25,25 \\
\hline 8 & $1,10(\mathrm{~s}, 3 \mathrm{H})$ & 27,84 \\
\hline 9 & $1,34(\mathrm{~s}, 3 \mathrm{H})$ & 24,23 \\
\hline
\end{tabular}




\section{III.4.CÁLCULOS DE MODELAGEM MOLECULAR PARA OS COMPOSTOS}




\section{III.4. CÁlCULOS DE MODELAGEM MOLECULAR PARA OS COMPOSTOS}

Com a intenção de procurar uma possível relação entre os valores de ${ }^{4} \mathbf{J}_{\mathrm{HH}}$ medidos e a estrutura molecular, fizemos cálculos de modelagem molecular para determinar os ângulos diedros entre os hidrogênios envolvidos nos acoplamentos a longa distância.

Os estudos de modelagem molecular dos compostos foram feitos utilizando-se vários programas de mecânica molecular, ${ }^{28-33} \mathrm{com}$ o intuito de observar os diferentes resultados fornecidos e analisar o que melhor respondia aos nossos interesses, haja visto que estes programas possuem algumas diferenças na base teórica utilizada para fazerem seus cálculos. Dentre os vários métodos de modelagem molecular existentes atualmente, utilizamos: método da mecânica molecular, método semi-empírico de orbital molecular, método $a b$ initio de orbital molecular.

O método da mecânica molecular é indicado para moléculas com centenas e milhares de átomos e é a única opção real para o estudo de grandes moléculas orgânicas e de biopolímeros. O método semi-empírico de orbital molecular permite tratar não só moléculas estáveis como o método da mecânica molecular, mas também, de produtos reativos ou estados de transição de modo tão fácil como de moléculas estáveis; estes métodos semi-empíricos são uma mistura baseada nas informações do referencial teórico da mecânica quântica com aproximações e parâmetros empíricos. O método $a b$ initio de orbital molecular constitui o principal caminho atualmente para a investigação da estrutura molecular e energética, contudo implica em um tempo de cálculo computacional enorme, uma vez que não usa aproximações ou parâmetros empíricos para a realização de seus cálculos como nos métodos semi-empíricos, mas sim, estima todos os valores que descrevem as funções de onda dos orbitais moleculares. $^{34}$ 
Iniciamos os estudos de modelagem molecular realizando uma busca conformacional para todos os compostos, utilizando-se o programa PC Model. Utilizamos então, o programa Boltz ${ }^{35}$ que usa a distribuição de boltzman para calcular o porcentagem de cada confôrmero, podendo assim, alimentar os programas Gaussian 98 (método ab initio) e Cs Mopac Pro (método semiempírico) com diversas conformações de cada composto, afim de elevar a confiabilidade dos resultados dos cálculos.

$\mathrm{Na}$ parte inicial do trabalho os cálculos também foram realizados no programa Gmmx (método de mecânica molecular), mas devido às limitações apresentadas, como não realizar cálculos em moléculas com ciclos de 3 membros (ex: epóxidos) decidimos por não mais o utilizar.

Nos cálculos realizados no programa Gaussian 98W utilizou-se o método HF e o conjunto de bases 6-31G(D), no programa Cs Mopac Pro foi utilizado o hamiltoniano semi-empírico AM1 e no programa PCModel o campo de força MMX.

A geometria otimizada foi usada para determinar os valores dos ângulos diedros entre os hidrogênios presentes no anel dos compostos analisados e, posteriormente foram correlacionados com os valores de ${ }^{4} J_{\mathrm{HH}}$ experimentais.

A primeira constatação foi a confirmação daquilo que já havíamos antes notado: ocorre acoplamento ${ }^{4} \mathrm{~J}_{\mathrm{HH}} \neq 0$ mesmo quando a conformação em "W" (planar) não é possível. Mais importante, porém, é que há uma relação aparentemente racionalizável entre os valores de ${ }^{4} \mathrm{~J}_{\mathrm{HH}}$ e os ângulos diedros envolvidos. Como mostrado na Figura 2, o acoplamento entre H1 e H2 ocorre através das quatro ligações $\sigma$ definidas pelo caminho H1-C1-C2-C3-H2 e envolve dois ângulos diedros $\theta_{1}$ e $\theta_{2}$. 


\section{Figura 2}

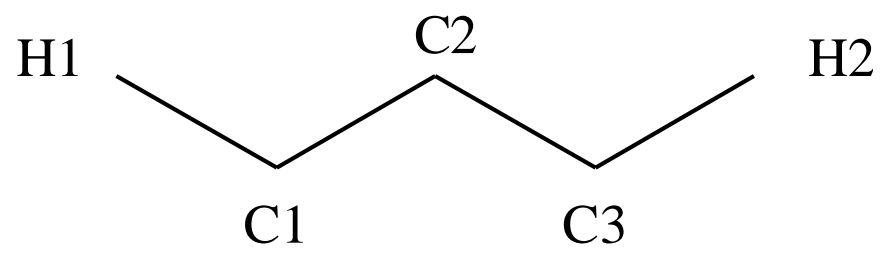

2 ângulos diedros:

$\theta_{1}: \mathrm{H} 1, \mathrm{C} 1, \mathrm{C} 2, \mathrm{C} 3$

altera-se por rotação

da ligação $\mathrm{C} 1$ - C2

$\theta_{2}: \mathrm{C} 1, \mathrm{C} 2, \mathrm{C} 3, \mathrm{H} 2$

altera-se por rotação

da ligação C2 - C3

A tabela abaixo mostra os valores de ${ }^{4} \mathrm{~J}_{\mathrm{HH}}(\mathrm{Hz})$ medidos, os hidrogênios envolvidos nos acoplamentos e os ângulos $\theta_{1}$ e $\theta_{2}$ calculados nos diversos programas computacionais.

\begin{tabular}{|c|c|c|c|c|c|c|c|c|c|}
\hline \multirow{2}{*}{ Entry } & \multirow{2}{*}{ Hidrogênios } & \multirow{2}{*}{ Composto } & \multirow{2}{*}{${ }^{4} \mathrm{~J}(\mathrm{~Hz})$} & \multicolumn{2}{|c|}{$\begin{array}{l}\text { Ângulos Diedros } \\
\text { (PcModel)* }\end{array}$} & \multicolumn{2}{|c|}{$\begin{array}{l}\text { Ângulos Diedros } \\
\text { (Cs Mopac Pro)* }\end{array}$} & \multicolumn{2}{|c|}{$\begin{array}{c}\text { Ângulos Diedros } \\
\text { (Gaussian98)* }\end{array}$} \\
\hline & & & & $\theta_{1}$ & $\theta_{2}$ & $\theta_{1}$ & $\theta_{2}$ & $\theta_{1}$ & $\theta_{2}$ \\
\hline 1 & & $7 \mathrm{a}$ & 2,6 & 120,09 & 130,55 & 128,98 & 140,24 & 131,26 & 144,01 \\
\hline 2 & & $8 \mathrm{~b}$ & 2,6 & 130,43 & 134,67 & 122,77 & 136,40 & 129,35 & 143,98 \\
\hline 3 & & 2 & 2,2 & 125,80 & 128,70 & 144,17 & 142,61 & 175,89 & 160,68 \\
\hline
\end{tabular}




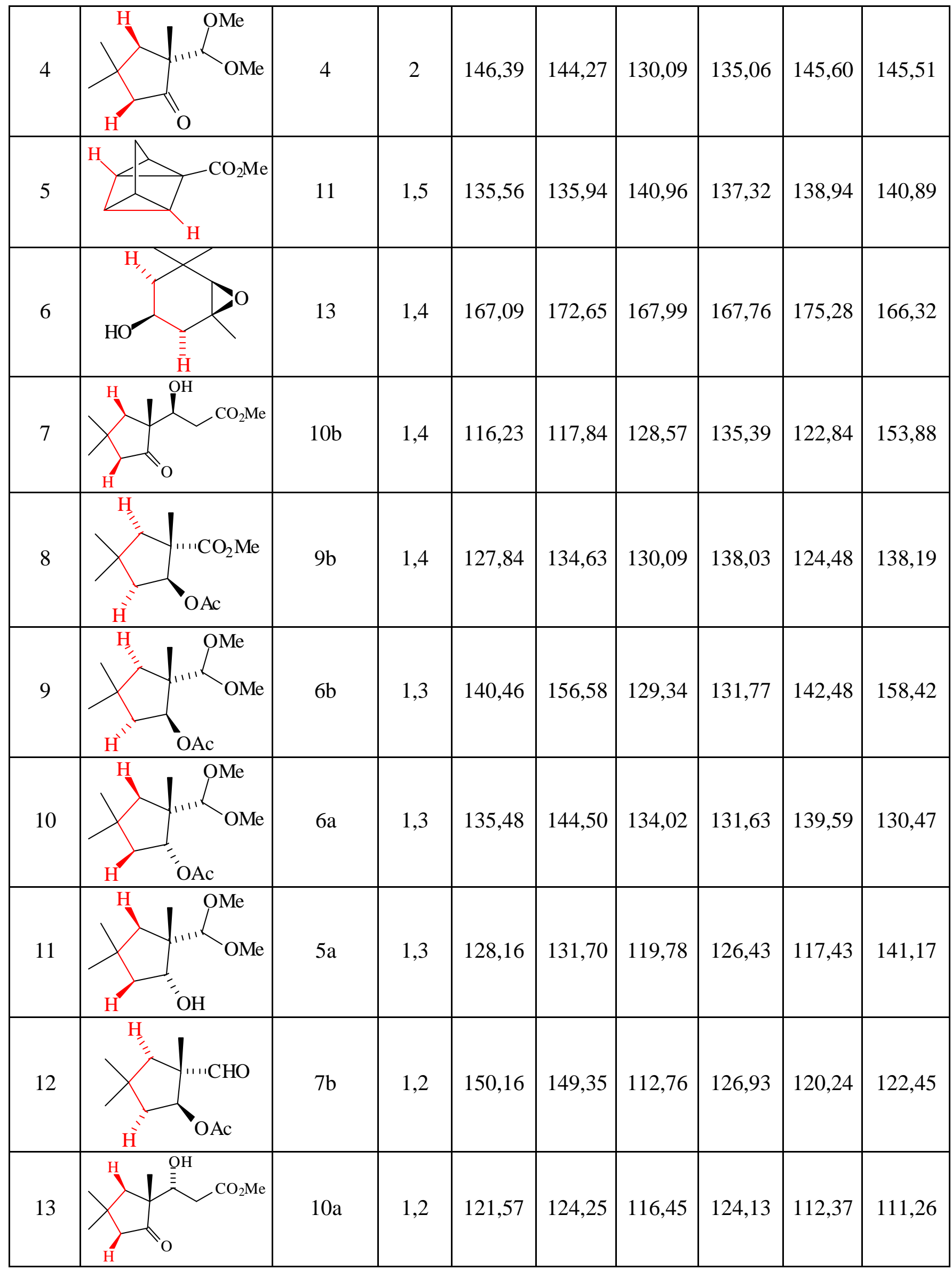




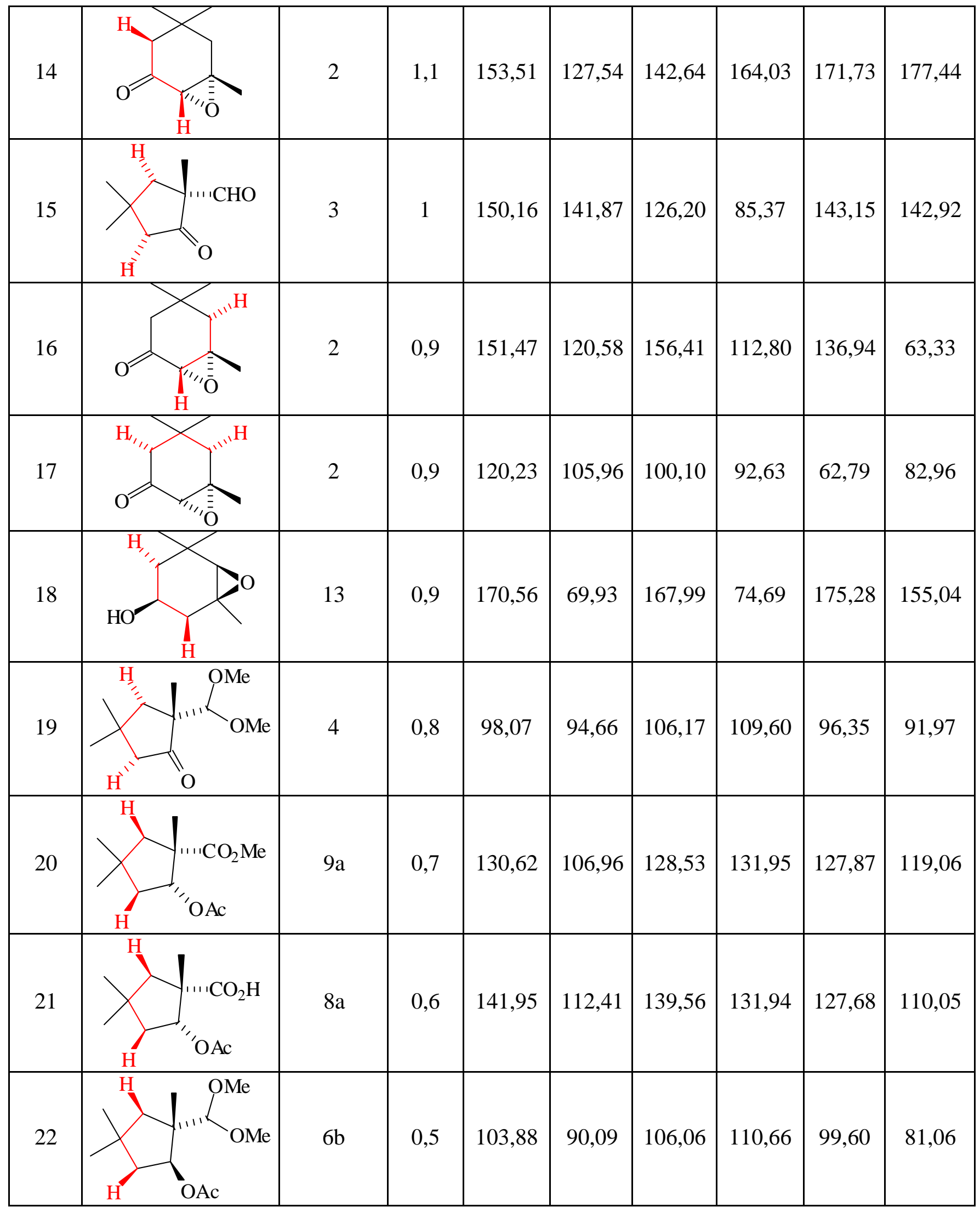




\begin{tabular}{|c|c|c|c|c|c|c|c|c|}
\hline 23 & 3 & 0 & 93,18 & 95,11 & 91,97 & 93,62 & 97,94 & 94,72 \\
\hline 24 & & & & & & & & \\
\hline
\end{tabular}

* Esses ângulos são médias ponderadas (de acordo com a abundância do confôrmero) dos ângulos dos vários confôrmeros possíveis. Sua finalidade é apenas de facilitar a visualização da relação entre os valores de $\mathrm{J}$ e os valores dos ângulos. A relação matemática, segundo nos parece, é através da média do produto dos quadrados dos cossenos, e o valor médio aqui apresentado não pode ser usado em cálculos. 
No intuito de estudar a relação entre os valores de ${ }^{4} J_{\mathrm{HH}}$ e os ângulos diedros envolvidos, foram calculados os cossenos dos ângulos $\theta_{1}$ e $\theta_{2}$ e a média dos produtos dos quadrados dos cossenos destes ângulos (levando-se em conta a contribuição de cada confôrmero) foram relacionados com os valores de ${ }^{4} \mathbf{J}_{\mathrm{HH}}$ obtidos experimentalmente.

De fato, uma simples extensão da equação de Karplus ${ }^{36}$ (segundo a qual ${ }^{3} \mathrm{~J}_{\mathrm{HH}}$ é aproximadamente proporcional ao quadrado do cosseno do ângulo diedro envolvido) resulta em uma correlação bastante razoável entre os valores de ${ }^{4} \mathbf{J}_{\mathrm{HH}}$ e a média dos produtos dos quadrados dos cossenos dos ângulos diedros envolvidos, como mostrado nos Gráficos 1, 2 e 3

Tabela 54- Dados de ${ }^{4} \mathbf{J}_{\mathrm{HH}}(\mathrm{Hz})$ versus valores médios de $\cos ^{2} \theta_{1} \cdot \cos ^{2} \theta_{2}$ através de ângulos diedros obtidos pelo programa PCModel.

\begin{tabular}{|c|c|c|}
\hline Entry & ${ }^{4} \mathrm{~J}(\mathrm{~Hz})$ & $\cos ^{2} \theta_{1} \cdot \cos ^{2} \theta_{2}$ \\
\hline 1 & 2,6 & 0,14414 \\
\hline 2 & 2,6 & 0,211625 \\
\hline 3 & 2,2 & 0,54776 \\
\hline 4 & 2 & 0,540998 \\
\hline 5 & 1,5 & 0,26357 \\
\hline 6 & 1,4 & 0,936517 \\
\hline 7 & 1,4 & 0,287425 \\
\hline 8 & 1,4 & 0,185902 \\
\hline 9 & 1,3 & 0,504569 \\
\hline 10 & 1,3 & 0,34541 \\
\hline 11 & 1,3 & 0,180477 \\
\hline 12 & 1,2 & 0,616899 \\
\hline 13 & 1,2 & 0,05996 \\
\hline 14 & 1,1 & 0,564455 \\
\hline 15 & 1 & 0,593147 \\
\hline 16 & 0,9 & 0,220411 \\
\hline 17 & 0,9 & 0,423266 \\
\hline 18 & 0,9 & 0,142363 \\
\hline 19 & 0,8 & 0,052568 \\
\hline 20 & 0,7 & 0,044695 \\
\hline 21 & 0,6 & 0,096861 \\
\hline 22 & 0,5 & 0,020574 \\
\hline 23 & 0 & 0,024324 \\
\hline 24 & 0 & 0,003492 \\
\hline 25 & 0 & 0,000912 \\
\hline 26 & 0 & 0,012373 \\
\hline & & \\
\hline
\end{tabular}




\begin{tabular}{|l|l|l|}
\hline 27 & 0 & 0,011026 \\
\hline 28 & 0 & 0,003228 \\
\hline
\end{tabular}

Gráfico 1 - Dados de ${ }^{4} \mathrm{~J}_{\mathrm{HH}}(\mathrm{Hz})$ versus $\cos ^{2} \theta_{1} \cdot \cos ^{2} \theta_{2}$ através de

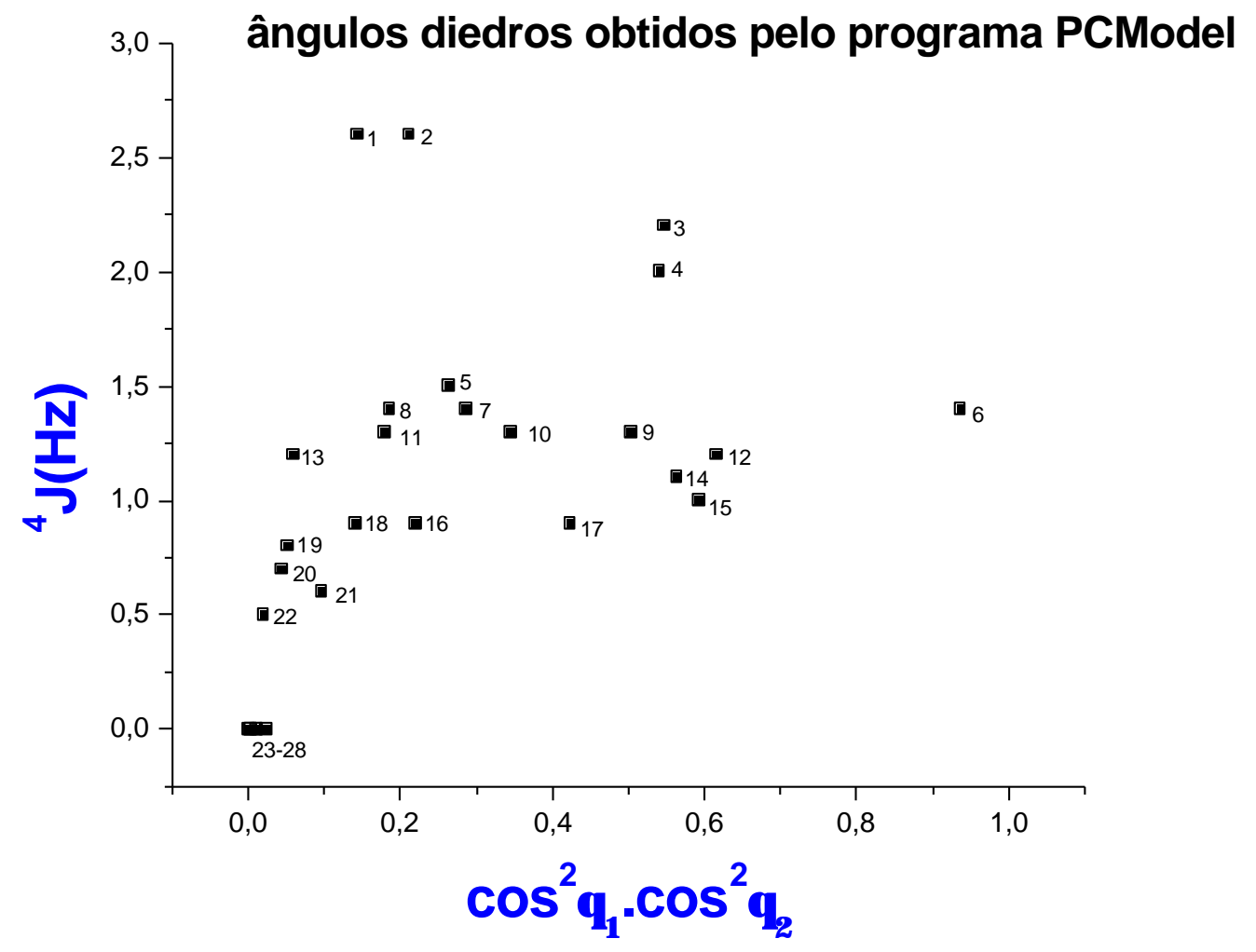


Tabela 55- Dados de ${ }^{4} \mathbf{J}_{\mathrm{HH}}(\mathrm{Hz})$ versus valores médios de $\cos ^{2} \theta_{1} \cdot \cos ^{2} \theta_{2}$ através de ângulos diedros obtidos pelo campo de força AM1 do programa Cs Mopac Pro.

\begin{tabular}{|c|c|c|}
\hline Entry & ${ }^{4} \mathrm{~J}(\mathrm{~Hz})$ & $\cos ^{2} \theta_{1} \cdot \cos ^{2} \theta_{2}$ \\
\hline 1 & 2,6 & 0,266556 \\
\hline 2 & 2,6 & 0,177654 \\
\hline 3 & 2,2 & 0,687096 \\
\hline 4 & 2 & 0,214338 \\
\hline 5 & 1,5 & 0,32661 \\
\hline 6 & 1,4 & 0,918527 \\
\hline 7 & 1,4 & 0,197746 \\
\hline 8 & 1,4 & 0,24755 \\
\hline 9 & 1,3 & 0,182517 \\
\hline 10 & 1,3 & 0,2262 \\
\hline 11 & 1,3 & 0,081141 \\
\hline 12 & 1,2 & 0,050091 \\
\hline 13 & 1,2 & 0,092856 \\
\hline 14 & 1,1 & 0,676148 \\
\hline 15 & 1 & 0,141261 \\
\hline 16 & 0,9 & 0,159101 \\
\hline 17 & 0,9 & 0,282001 \\
\hline 18 & 0,9 & 0,103994 \\
\hline 19 & $\overline{0,8}$ & 0,013348 \\
\hline 20 & 0,7 & 0,225482 \\
\hline 21 & 0,6 & 0,300985 \\
\hline 22 & 0,5 & 0,010796 \\
\hline 23 & 0 & 0,0072663 \\
\hline 24 & 0 & 0,018837 \\
\hline 25 & 0 & 0,044533 \\
\hline 26 & 0 & 0,013369 \\
\hline 27 & 0 & 0,015379 \\
\hline 28 & 0 & 0,014592 \\
\hline
\end{tabular}


Gráfico 2 - Dados de ${ }^{4} \mathrm{~J}_{\mathrm{HH}}(\mathrm{Hz})$ versus $\cos ^{2} \theta_{1} \cdot \cos ^{2} \theta_{2}$ através de ângulos diedros obtidos pelo programa Cs Mopac Pro Campo de força AM1

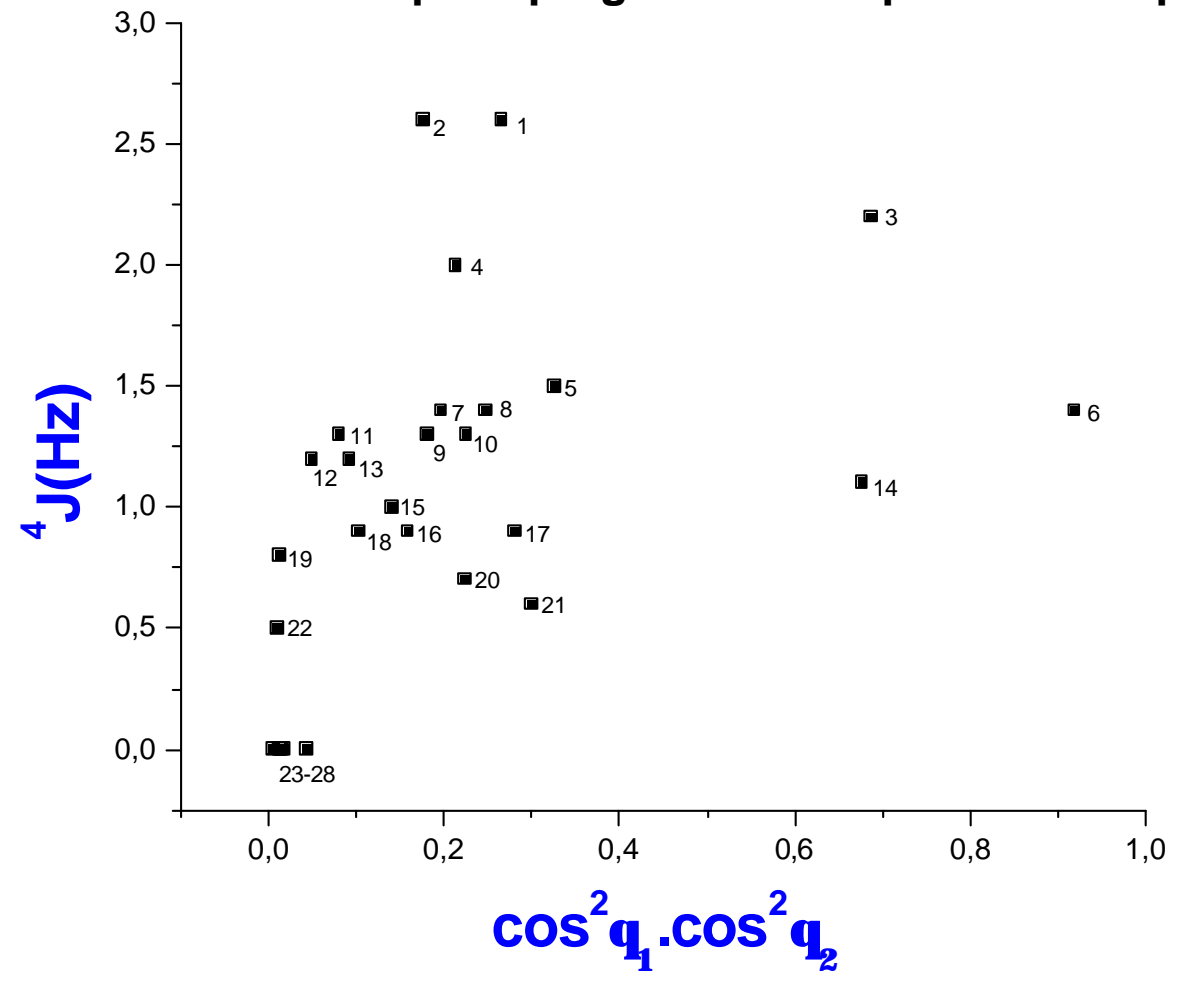


Tabela 56 - Dados de ${ }^{4} \mathrm{~J}_{\mathrm{HH}}(\mathrm{Hz})$ versus valores médios de $\cos ^{2} \theta_{1} \cdot \cos ^{2} \theta_{2}$ através de ângulos diedros obtidos pelo método HF e o conjunto de bases 6-31G(D) do programa Gaussian98.

\begin{tabular}{|c|c|c|}
\hline Entry & ${ }^{4} \mathrm{~J}(\mathrm{~Hz})$ & $\cos ^{2} \theta_{1} \cdot \cos ^{2} \theta_{2}$ \\
\hline 1 & 2,6 & 0,292084 \\
\hline 2 & 2,6 & 0,275732 \\
\hline 3 & 2,2 & 0,889492 \\
\hline 4 & 2 & 0,55944 \\
\hline 5 & 1,5 & 0,342679 \\
\hline 6 & 1,4 & 0,937814 \\
\hline 7 & 1,4 & 0,330652 \\
\hline 8 & 1,4 & 0,276282 \\
\hline 9 & 1,3 & 0,54411 \\
\hline 10 & 1,3 & 0,246098 \\
\hline 11 & 1,3 & 0,138898 \\
\hline 12 & 1,2 & 0,072553 \\
\hline 13 & 1,2 & 0,251222 \\
\hline 14 & 1,1 & 0,960951 \\
\hline 15 & 1 & 0,565368 \\
\hline 16 & $\overline{0,9}$ & 0,147064 \\
\hline 17 & 0,9 & 0,033813 \\
\hline 18 & $\overline{0,9}$ & 0,806555 \\
\hline 19 & 0,8 & 0,064988 \\
\hline 20 & 0,7 & 0,09612 \\
\hline 21 & 0,6 & 0,099182 \\
\hline 22 & 0,5 & 0,000672 \\
\hline 23 & 0 & 0,010493 \\
\hline 24 & 0 & 0,006719 \\
\hline 25 & 0 & 0,000824 \\
\hline 26 & 0 & 0,014325 \\
\hline 27 & 0 & 0,018939 \\
\hline 28 & 0 & 0,005421 \\
\hline
\end{tabular}


Gráfico 3-Dados de ${ }^{4} \mathrm{~J}_{\mathrm{HH}}(\mathrm{Hz}) \times \cos ^{2} \theta_{1} \cdot \cos ^{2} \theta_{2}$ através de ângulos diedros obtidos pelo método HF e o conjunto de bases 6-31G(D) do programa Gaussian98

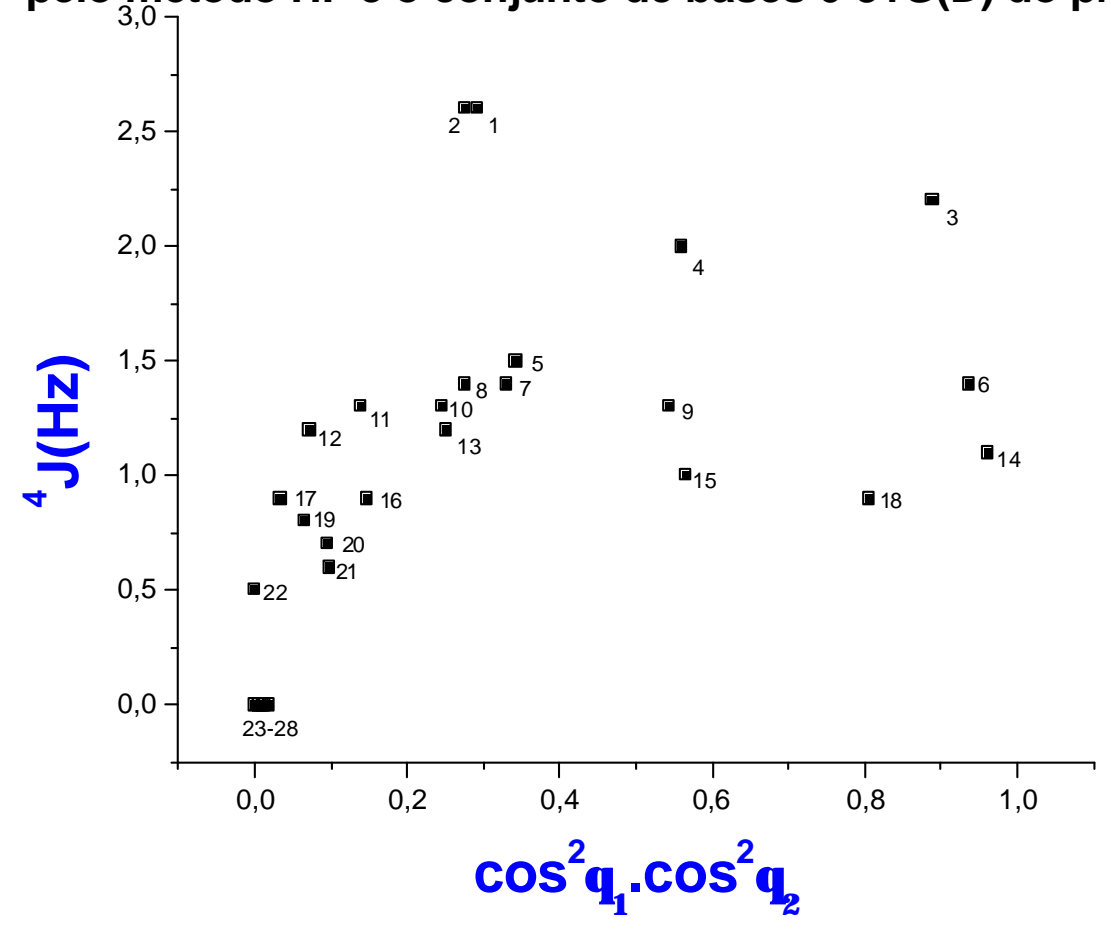




\section{Gráfico 4 - Gráficos 1, 2 e 3 sobrepostos}

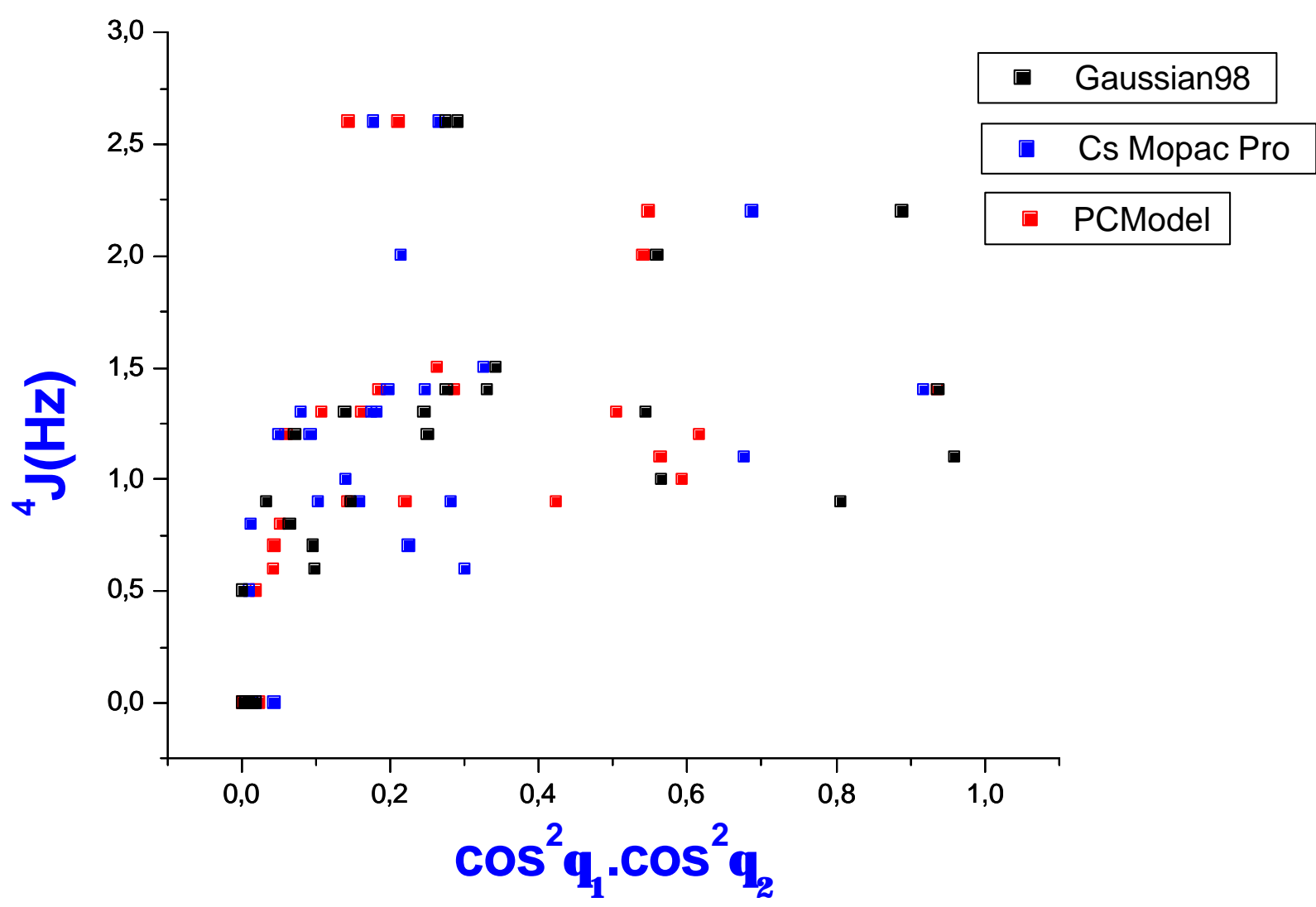


$\mathrm{Na}$ verdade os pontos não se alinham com perfeição sobre uma curva contínua, mas mostram certa tendência de aumento do valor de ${ }^{4} \mathbf{J}_{\mathrm{HH}}$ conforme os ângulos $\theta_{1}$ e $\theta_{2}$ se afastam de $90^{\circ}$ e se aproximam de $180^{\circ}$. Considerando o número relativamente pequeno de pontos calculados e os consideráveis erros relativos em medidas de valores de $\mathrm{J}$ tão pequenos, podemos considerar a correlação como boa, principalmente quando se toma em conta que vários fatores (como a eletronegatividade e orientação de substituintes, etc.) afetam fortemente o valor das constantes de acoplamento ${ }^{3} \mathrm{~J}_{\mathrm{HH}},{ }^{37}$ levando os químicos a formularem muitas variações da equação de Karplus ${ }^{38}$ para obter correlações razoáveis entre valores calculados e experimentais.

A diferença entre os gráficos 1, 2 e 3, melhor visualizada no gráfico 4, mostra que os métodos de cálculos dos ângulos não fornecem resultados com a desejável confiabilidade, mas apesar disso, pode-se observar uma tendência de aumento do valor de ${ }^{4} J_{H H}$ conforme os ângulos $\theta_{1}$ e $\theta_{2}$ se afastam de $90^{\circ}$ e se aproximam de $180^{\circ}$, mostrando que o acoplamento ${ }^{4} \mathrm{~J}_{\mathrm{HH}}$ é função do ângulo diedro entre os hidrogênios envolvidos no acoplamento. Como observado nos gráficos 1, 2 e 3 alguns pontos estão bem fora de uma curva contínua, o que mostra claramente que existem outros fatores afetando a constante de acoplamento ${ }^{4} \mathrm{~J}_{\mathrm{HH}}$ e que não foram tomados em consideração devido ao reduzido número de pontos.

Em um estudo desta natureza, podem ser cometidos vários erros relativos, que podem afetar o resultado final, como por exemplo: erros nas medidas de valores de $\mathrm{J}$ tão pequenos, devido a resolução do aparelho e os erros referentes aos valores do ângulos calculados pelos programas computacionais. Como observado nos gráficos apresentados, os valores dos ângulos diedros calculados diferem de um método para outro, sendo esta diferença maior para alguns pontos. Pensando nisto, resolvemos fazer uma estimativa de erros, usando o limite da resolução do aparelho em que os espectros de RMN foram obtidos $(0,12 \mathrm{~Hz})$, como o erro nas medidas de ${ }^{4} \mathrm{~J}_{\mathrm{HH}}$ e para os valores dos ângulos diedros 
calculados foi feita uma média aritimética entre o maior e o menor valor da média dos produtos dos quadrados dos cossenos dos diferentes métodos, tendo como erro relativo a diferença entre a média encontrada e o maior ou menor valor. $\mathrm{O}$ valor encontrado na média aritimética foi plotado em relação aos valores de ${ }^{4} \mathbf{J}_{\mathrm{HH}}$, como observado no gráfico 5 .

Tabela 57 - Dados de ${ }^{4} \mathbf{J}_{\mathrm{HH}}(\mathrm{Hz})$, média de $\cos ^{2} \theta_{1} \cdot \cos ^{2} \theta_{2}$ dos diferentes programas, média aritimética e o erro relativo para cada um dos pontos.

\begin{tabular}{|c|c|c|c|c|c|c|}
\hline Entry & ${ }^{4} \mathrm{~J}(\mathrm{~Hz})$ & $\begin{array}{c}\text { PCModel } \\
\cos ^{2} \theta_{1} \cdot \cos ^{2} \theta_{2}\end{array}$ & $\begin{array}{c}\text { Cs Mopac Pro } \\
\cos ^{2} \theta_{1} \cdot \cos ^{2} \theta_{2}\end{array}$ & $\begin{array}{c}\text { Gaussian98 } \\
\cos ^{2} \theta_{1} \cdot \cos ^{2} \theta_{2}\end{array}$ & $\begin{array}{c}\text { Média } \\
\text { Aritimética }\end{array}$ & Erro \\
\hline 1 & 2,6 & 0,14414 & 0,266556 & 0,292084 & 0,218112 & 0,073972 \\
\hline 2 & 2,6 & 0,211625 & 0,177654 & 0,275732 & 0,226693 & 0,049039 \\
\hline 3 & 2,2 & 0,54776 & 0,687096 & 0,889492 & 0,718626 & 0,170866 \\
\hline 4 & 2 & 0,540998 & 0,214338 & 0,55944 & 0,386889 & 0,172551 \\
\hline 5 & 1,5 & 0,26357 & 0,32661 & 0,342679 & 0,303125 & 0,039555 \\
\hline 6 & 1,4 & 0,936517 & 0,918527 & 0,937814 & 0,928171 & 0,009644 \\
\hline 7 & 1,4 & 0,287425 & 0,197746 & 0,330652 & 0,264199 & 0,066453 \\
\hline 8 & 1,4 & 0,185902 & 0,24755 & 0,276282 & 0,231092 & 0,04519 \\
\hline 9 & 1,3 & 0,504569 & 0,182517 & 0,54411 & 0,363314 & 0,180797 \\
\hline 10 & 1,3 & 0,34541 & 0,2262 & 0,246098 & 0,285805 & 0,059605 \\
\hline 11 & 1,3 & 0,180477 & 0,081141 & 0,138898 & 0,130809 & 0,049668 \\
\hline 12 & 1,2 & 0,616899 & 0,050091 & 0,072553 & 0,333495 & 0,283404 \\
\hline 13 & 1,2 & 0,05996 & 0,092856 & 0,251222 & 0,155591 & 0,095631 \\
\hline 14 & 1,1 & 0,564455 & 0,676148 & 0,960951 & 0,762703 & 0,198248 \\
\hline 14 & 1 & 0,593147 & 0,141261 & 0,565368 & 0,367204 & 0,225943 \\
\hline 16 & 0,9 & 0,220411 & 0,159101 & 0,147064 & 0,183738 & 0,036674 \\
\hline 17 & 0,9 & 0,423266 & 0,282001 & 0,033813 & 0,22854 & 0,194727 \\
\hline 18 & 0,9 & 0,142363 & 0,103994 & 0,806555 & 0,455275 & 0,351281 \\
\hline 19 & 0,8 & 0,052568 & 0,013348 & 0,064988 & 0,039168 & 0,02582 \\
\hline 20 & 0,7 & 0,044695 & 0,225482 & 0,09612 & 0,135089 & 0,090394 \\
\hline 21 & 0,6 & 0,096861 & 0,300985 & 0,099182 & 0,198923 & 0,102062 \\
\hline 22 & 0,5 & 0,020574 & 0,010796 & 0,000672 & 0,010623 & 0,009951 \\
\hline 23 & 0 & 0,024324 & 0,0072663 & 0,010493 & 0,015795 & 0,008529 \\
\hline 24 & 0 & 0,003492 & 0,018837 & 0,006719 & 0,005106 & 0,001614 \\
\hline 25 & 0 & 0,000912 & 0,044533 & 0,000824 & 0,022723 & 0,021899 \\
\hline 26 & 0 & 0,012373 & 0,013369 & 0,014325 & 0,013349 & 0,000976 \\
\hline 27 & 0 & 0,011026 & 0,015379 & 0,018939 & 0,014983 & 0,003957 \\
\hline 28 & 0 & 0,003228 & 0,014592 & 0,005421 & 0,00891 & 0,005682 \\
\hline
\end{tabular}


Gráfico 5 - Estimativa de erros nos valores de ${ }^{4} \mathrm{~J}_{\mathrm{HH}}(\mathrm{Hz})$ e nos valores de $\theta_{1}$ e $\theta_{2}$ calculados nos diferentes métodos de modelagem

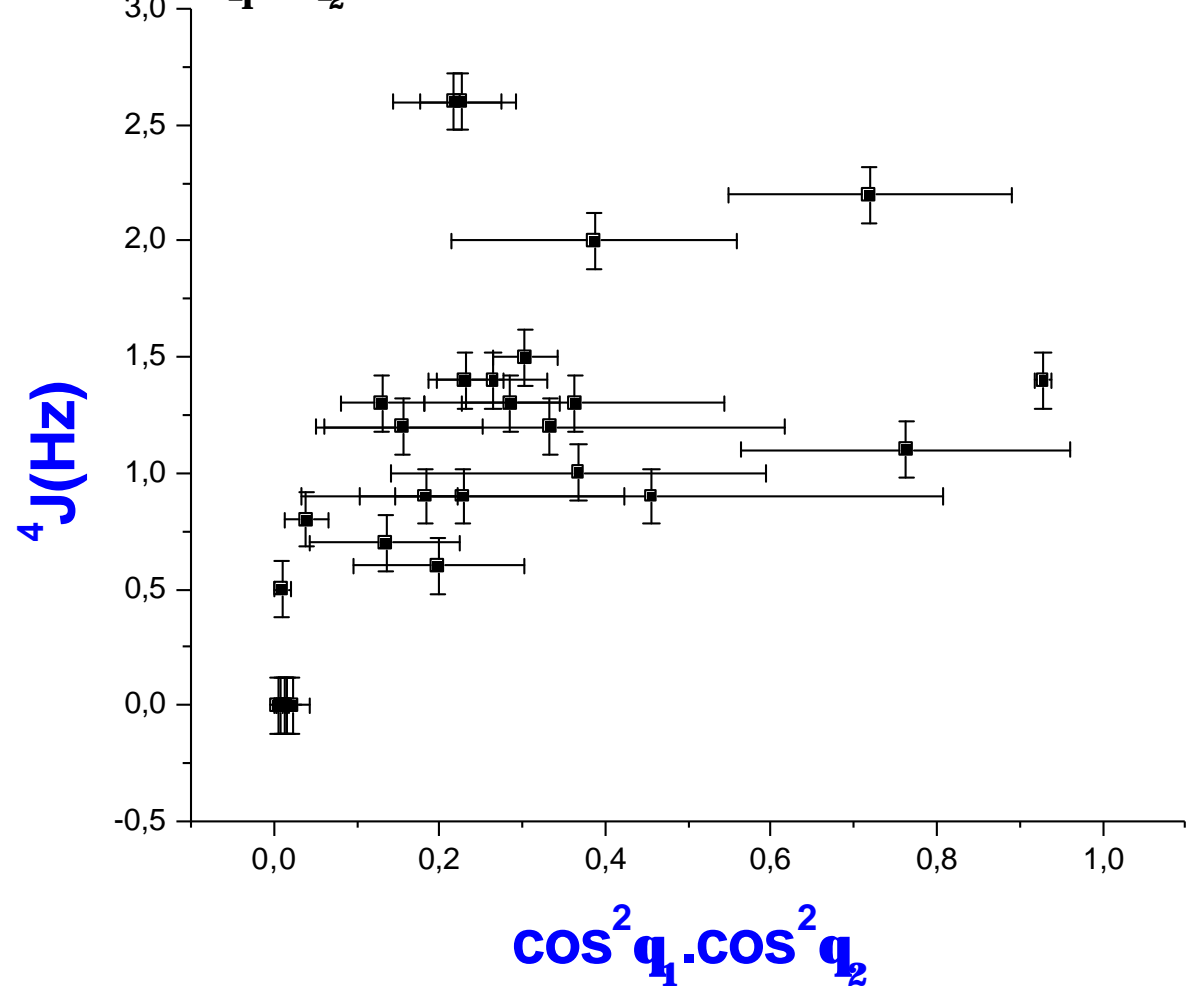


Os estudos de RMN do composto 12 revelaram ainda, 3 constantes de acoplamento a longa distância ${ }^{4} \mathrm{~J}_{\mathrm{HH}}$, contudo, esses dados não foram inseridos nas tabelas e gráficos apresentados, pois se trata de acoplamentos a longa distância entre hidrogênios alílicos, envolvendo 3 ligações simples e uma ligação dupla. A tabela abaixo mostra os hidrogênios envolvidos nos acoplamentos e os respectivos valores de ${ }^{4} \mathrm{~J}_{\mathrm{HH}}$.

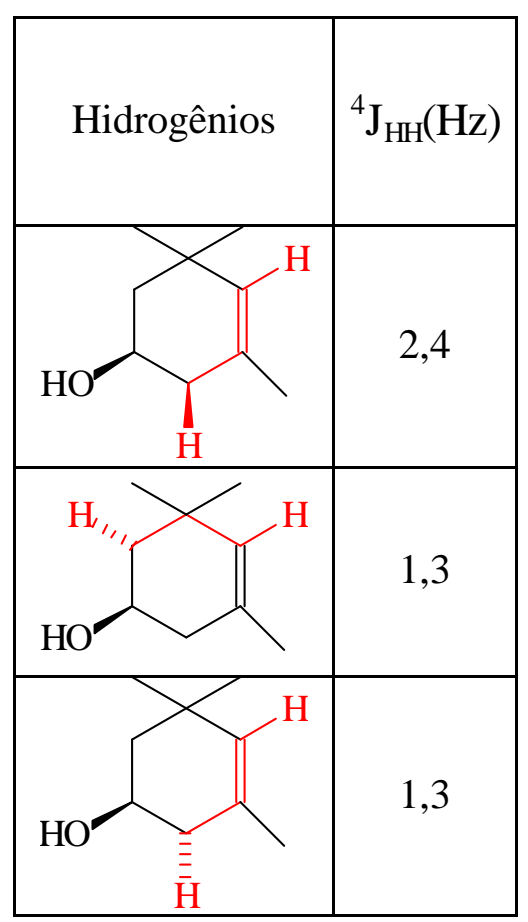

Segundo a literatura, ${ }^{39}$ nos acoplamentos a longa distância envolvendo hidrogênios alílicos, se tem um valor máximo de $\mathrm{J}$ quando $\theta=90^{\circ} \mathrm{e}$ não em 0 e $180^{\circ}$ como nos casos dos acoplamentos a longa distância envolvendo apenas ligações simples. 


\section{CONCLUSÃO}

Os intermediários sintéticos (ciclopentanos substituídos) foram preparados a partir de procedimentos já descritos na literatura. ${ }^{15}$ Procuramos, no entanto, otimizar os resultados, obtendo quantidades suficientes e com pureza adequada, conforme necessário para as análises de RMN.

O estudo detalhado de RMN dos compostos com a utilização de técnicas modernas tais como NOE DIFF, HMQC e "J-resolved", permitiu atribuir os sinais correspondentes a cada um dos hidrogênios de todos os compostos e estabelecer os valores dos deslocamentos químicos, das constantes de acoplamento, inclusive os acoplamentos a longa distância ${ }^{4} \mathbf{J}_{\mathrm{HH}}$, a posição na molécula e a estereoquímica relativa dos hidrogênios.

Observou-se que ocorre acoplamento ${ }^{4} \mathbf{J}_{\mathrm{HH}} \neq 0$ mesmo quando a conformação "W" planar não é possível. Mais importante, porém, é que existe uma relação entre os valores de ${ }^{4} \mathrm{~J}_{\mathrm{HH}}$ medidos e os ângulos diedros entre os hidrogênios envolvidos no acoplamento, calculados com auxílio de programas computacionais.

$\mathrm{O}$ estudo de modelagem molecular dos compostos através dos diferentes métodos permitiu analisar os diferentes resultados obtidos e fazer uma comparação entre eles. Os resultados mostram que os métodos de cálculos dos ângulos não fornecem resultados com a desejável confiabilidade. A diferença entre os gráficos apresentados evidencia a existência de outros fatores que não foram tomados em consideração e que podem afetar os valores de ${ }^{4} \mathrm{~J}_{\mathrm{HH}}$. Como consequiência, é natural que os pontos nos gráficos não se alinhem em uma curva contínua. Mas mesmo assim há uma certa tendência de aumento de ${ }^{4} \mathrm{~J}_{\mathrm{HH}}$ conforme os ângulos $\theta_{1}$ e $\theta_{2}$ se afastam de $90^{\circ}$ e se aproximam de $180^{\circ}$. 


\section{PARTE EXPERIMENTAL}




\section{V.PARTE EXPERIMENTAL}

\section{V.1.INTRODUÇÃO}

Neste trabalho preparamos vários intermediários sintéticos de sínteses anteriormente realizadas em nosso laboratório. ${ }^{15}$ Procuramos, no entanto, otimizar os resultados, obtendo quantidades suficientes e com pureza adequada para efetuar as análises planejadas .

Os espectros de ressonância magnética nuclear de próton $\left({ }^{1} \mathrm{H}-\mathrm{RMN}\right)$ foram registrados em um espectrômetro Bruker DPX-300. Os deslocamentos químicos ( $\delta)$ estão relatados em parte por milhão ( $\mathrm{ppm}$ ) em relação ao tetrametilsilano (TMS), utilizado como padrão interno, colocando-se entre parênteses a multiplicidade $(\mathrm{s}=$ singleto, $\mathrm{s} .1=$ singleto largo, $\mathrm{d}=$ dubleto, $\mathrm{t}=$ tripleto, $\mathrm{q}=$ quadrupleto, $\mathrm{d} . \mathrm{d}=$ duplo dubleto, d.d.d = duplo duplo dubleto, d.d.d.d $=$ duplo duplo duplo dubleto, d.t $=$ duplo tripleto, d.d.t $=$ duplo duplo tripleto, $\mathrm{d} . \mathrm{q}=$ duplo quadrupleto, $\mathrm{m}=$ multipleto), $\mathrm{a}$ constante de acoplamento $(\mathrm{J})$ em Hertz $(\mathrm{Hz})$ e o número de hidrogênios deduzidos da integral relativa.

Os espectros de ressonância magnética nuclear de carbono-13 $\left({ }^{13} \mathrm{C}\right.$ RMN) foram registrados a $75 \mathrm{MHz}$ em um espectrômetro Bruker DPX-300 e foram traçados de acordo com a conveniência, utilizando-se as seguintes técnicas:

BB - Broad Band;

DEPT - Distortionless Enhancement by Polarization Transfer.

Os espectros de absorção no infravermelho foram registrados em um espectrofotômetro Perkin-Elmer modelo 1600-FT, em celas de $\mathrm{KBr}$ para líquidos (filme) ou em pastilhas de $\mathrm{KBr}$ para sólidos.

Os espectros de massas de baixa resolução foram obtidos em um aparelho HP GC/MS SYSTEM 5988-A por injeção das amostras através de um cromatógrafo gasoso (coluna capilar HP-5 de $25 \mathrm{~m}, 0,53 \mathrm{~mm}$ de diâmetro e espessura do filme de 0,23 $\mathrm{mm}$ de diâmetro, ligeiramente apolar), utilizando-se energia de ionização de $70 \mathrm{eV}$. Os íons fragmentados foram descritos como relação entre massa e carga $(\mathrm{m} / \mathrm{z})$ e a abundância relativa expressa em porcentagem. 
As análises por cromatografia gasosa foram realizadas em um aparelho Varian GC 3400 com uma coluna capilar de sílica fundida (30 m de comprimento $\times$ $0.25 \mathrm{~mm}$ de diâmetro) contendo DB 1701 (fase sólida de espessura $0.25 \mu \mathrm{m}$ ) operando na faixa de temperaturas de $50-200^{\circ} \mathrm{C}$.

Para destilação horizontal empregou-se um aparelho de destilação evaporativa horizontal Kugelrohrofen Büchi modelo GKR-50. As temperaturas registradas referem-se à temperatura do forno.

Para concentrar as soluções orgânicas foram utilizados evaporadores rotatórios do tipo Buchler e Büchi, operando sob pressão reduzida de aproximadamente $20 \mathrm{mmHg}$.

As análises por cromatografia em camada delgada (ccd) foram feitas com placas de sílica gel 60 da Merck®. Purificações por cromatografia em coluna foram realizadas utilizando sílica gel 60 da Merck®.

Os solventes e reagentes comerciais foram convenientemente purificados conforme métodos usuais. ${ }^{40}$

\section{Preparo das Amostras para Medidas de Efeito Noe}

O efeito NOE é bastante seletivo e para sua obtenção é necessário se ter algumas precauções como: não lavar os tubos de ressonância com sulfocrômica ou qualquer substância paramagnética, desagueificar a amostra para retirar o oxigênio dissolvido, calibrar o aparelho e usar $\pm 0,6 \mathrm{~mL}$ de solvente em $5-10 \mathrm{mg}$ de substrato. 


\section{V.2.REAÇÕES DESCRITAS}

1)<smiles>CC1=CC(=O)CC(C)(C)C1</smiles>

1<smiles>CC1(C)CC(=O)C2OC2(C)C1</smiles>

2

2)<smiles>CC1(C)CC(=O)C2OC2(C)C1</smiles>

2

\section{$\mathrm{BF}_{3} \cdot \mathrm{OEt}_{2}$ \\ $\mathrm{CH}_{2} \mathrm{Cl}_{2}$}

3)<smiles>CC1(C)CC(=O)C(C)(C=O)C1</smiles>

3<smiles>CC1(C)CC(=O)C(C)(C=O)C1</smiles>

3

\section{$(\mathrm{MeO})_{3} \mathrm{CH}$ PTSA}

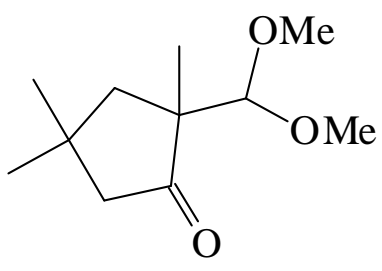

4 
4)<smiles>COC(OC)C1(C)CC(C)(C)CC1=O</smiles>

4

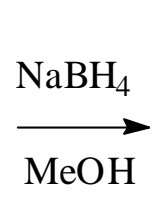<smiles>COC(OC)[C@]1(C)CC(C)(C)C[C@H]1O</smiles>

$5 a$<smiles>COC(OC)[C@@]1(C)CC(C)(C)C[C@H]1O</smiles>

$\mathbf{5 b}$

5)<smiles>COC(OC)[C@]1(C)CC(C)(C)C[C@H]1O</smiles>

$5 a$
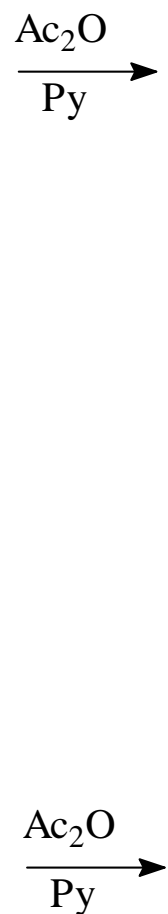<smiles>COC(OC)[C@@]1(C)CC(C)(C)C[C@H]1OC(C)=O</smiles>

$6 a$

6)<smiles>COC(OC)[C@]1(C)CC(C)(C)C[C@H]1O</smiles>

$\mathbf{5 b}$<smiles>COC(OC)[C@@]1(C)CC(C)(C)C[C@H]1OC(C)C</smiles>

6b 
7)<smiles>COC(OC)[C@]1(C)CC(C)(C)C[C@H]1OC(C)=O</smiles>

$6 a$

8)<smiles>COC(OC)[C@@]1(C)CC(C)(C)CC1OC(C)=O</smiles>

6b

9)<smiles>CC(=O)O[C@H]1CC(C)(C)C[C@]1(C)C=O</smiles>

$7 a$

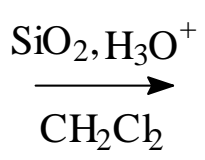<smiles>CC(=O)O[C@H]1CC(C)(C)C[C@]1(C)C=O</smiles>

$7 a$

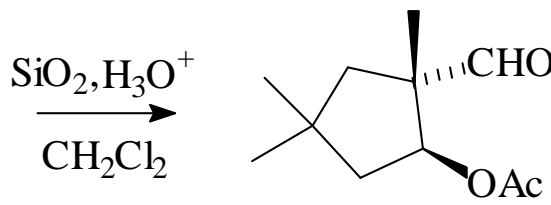

7b

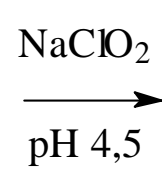

$\overrightarrow{\mathrm{pH}} 4,5$
$8 \mathbf{a}$ 
10)<smiles>CC(=O)O[C@H]1CC(C)(C)C[C@]1(C)C=O</smiles>

7b

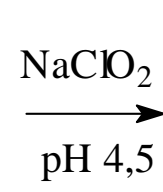

$\mathrm{pH} 4,5$

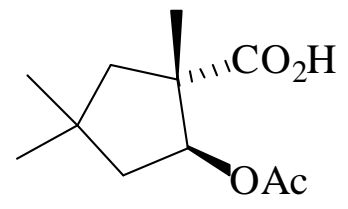

8b

11)<smiles>CC(=O)O[C@H]1CC(C)(C)C[C@]1(C)C(=O)O</smiles>

$8 a$

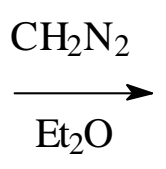

$\mathrm{Et}_{2} \mathrm{O}$<smiles></smiles>

9a

12)<smiles>CC(=O)O[C@H]1CC(C)(C)C[C@]1(C)C(=O)O</smiles>

$8 b$

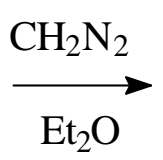

$\mathrm{Et}_{2} \mathrm{O}$

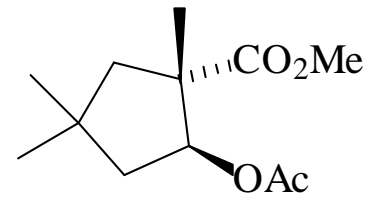

$9 b$ 
13)

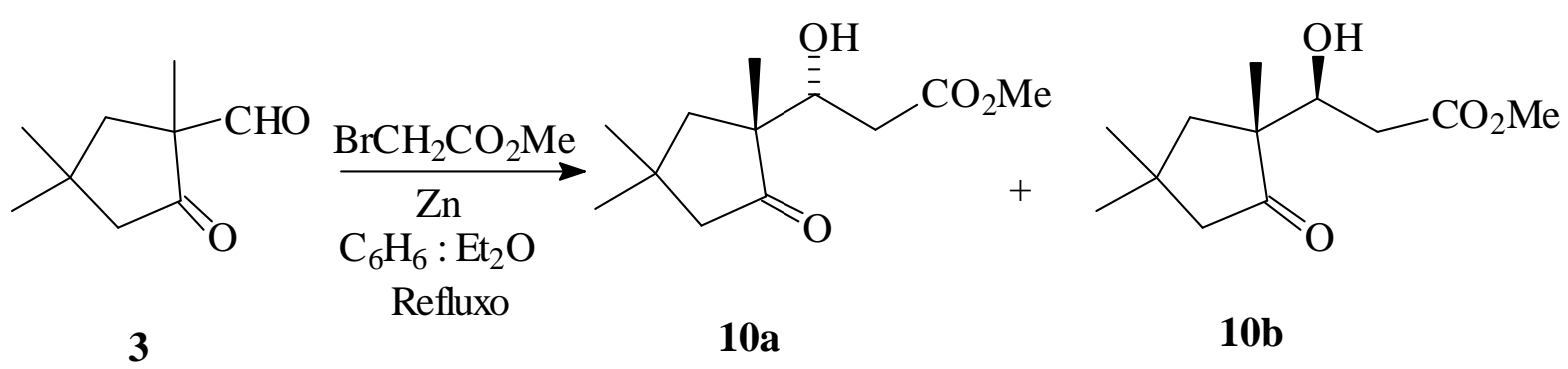




\section{V.3.PROCEDIMENTO EXPERIMENTAL}

\section{V.3.1.PREPARAÇÃO DO ( $( \pm)$ 3,5,5-TRIMETIL-2,3-EPOXI-CICLOHEXANONA}

(2)

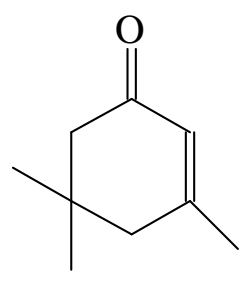

1

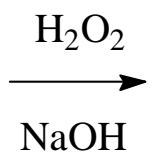

Em um balão de 3 bocas de $1 \mathrm{~L}$ equipado com um funil de adição, um agitador mecânico e um termômetro foi colocada uma solução de 55,2g (0,4 moles) de isoforona 1 e $115 \mathrm{ml}$ (1,2 moles) de solução aquosa de peróxido de hidrogênio $30 \%$ em $400 \mathrm{~mL}$ de metanol. Em seguida adicionou-se lentamente $33 \mathrm{~mL}(0,2$ moles $)$ de solução aquosa $6 \mathrm{~N} \mathrm{NaOH}$ gota a gota por um período de 1 hora. Durante a adição a temperatura da reação foi mantida entre 15 e $20^{\circ} \mathrm{C}$ resfriando com um banho de água gelada. Terminada a adição a reação foi mantida em agitação por 3 horas com temperatura entre 20 e $25^{\circ} \mathrm{C}$. A mistura reacional foi então colocada em $500 \mathrm{~mL}$ de água e a mistura resultante foi extraída com 2 porções de $400 \mathrm{~mL}$ de $\mathrm{Et}_{2} \mathrm{O}$. As frações orgânicas foram então lavadas com água e secadas com sulfato de magnésio. O solvente foi removido sob pressão reduzida e o produto foi destilado em um sistema de destilação horizontal. O rendimento da reação foi de 41,8-43g (68-70\%).

Dados espectroscópicos

$-\mathrm{RMN}{ }^{1} \mathrm{H}, 300 \mathrm{MHz}, \delta\left(\mathrm{CDCl}_{3}\right) \delta: \quad 2,93(\mathrm{dd}, 1 \mathrm{H}, \mathrm{Jec}=1,1 \mathrm{e} \mathrm{Jeb}=0,9 \mathrm{~Hz}) ; 2,51$

$$
\begin{aligned}
& (\mathrm{dd}, 1 \mathrm{H}, \mathrm{Jdc}=13,4 \text { e Jdb=0,9Hz); 1,97 }(\mathrm{dt}, 1 \mathrm{H}, \\
& \mathrm{Jba}=15 \text { e Jbd=Jbe=0,9 Hz); 1,69 (ddd, } \\
& 1 \mathrm{H}, \mathrm{Jcd}=13,4, \mathrm{Jca}=2,2 \text { e Jce=1,1Hz); 1,59 (dd, } \\
& 1 \mathrm{H}, \mathrm{Jab}=15 \text { e Jac=2,2Hz); 1,30 (s, 3H); 0,9 } \\
& \text { s, 3H); 0,8 (s,3H). }
\end{aligned}
$$

$-\mathrm{RMN}{ }^{13} \mathrm{C}, 75 \mathrm{MHz}, \delta\left(\mathrm{CDCl}_{3}\right): 207,68 ; 64,05 ; 61,17 ; 47,77 ; 42,51 ; 35,92 ; 30,61$;

$$
27,61 ; 23,82 \text {. }
$$

-IV (Filme Líquido), $v_{\max }: 2952 ; 2930 ; 2870 ; 1717 ; 1465 ; 1153 \mathrm{~cm}^{-1}$.

-EM, m / e, (abundância relativa, \%): 154 (4,5); 139 (24); 111 (10); 97 (24,5); 83 (100);

$$
69(60) ; 55 \text { (48). }
$$




\section{V.3.2. PREPARAÇÃO DO ( \pm )- 1,4,4- TRIMETIL-2-OXO-CICLOPENTANO-1- CARBOXALDEÍDO (3).}<smiles>CC1(C)CC(=O)C2OC2(C)C1</smiles>

2<smiles>CCOC(C)(CC)CC</smiles>

$65 \%$

Adicionou-se lentamente a solução de $(0,600 \mathrm{~g} ; 3.89$ mmoles $)$ da epoxiisoforona 2 e $1,0 \mathrm{~mL}$ de $\mathrm{CH}_{2} \mathrm{Cl}_{2}$ à um balão contendo $15 \mathrm{~mL}$ de $\mathrm{CH}_{2} \mathrm{Cl}_{2}$ e mais $0,3 \mathrm{~mL}$ de $\mathrm{BF}_{3} \cdot \mathrm{OEt}_{2}$. A mistura foi mantida em agitação durante 30 minutos à temperatura ambiente e em seguida hidrolizou-se com uma solução saturada de $\mathrm{NaHCO}_{3}$. As fases foram separadas e a fase orgânica foi lavada várias vezes com uma solução saturada de $\mathrm{NaHCO}_{3}$ e com uma solução de $\mathrm{NaCl}$, secou-se sob $\mathrm{MgSO}_{4}$ anidro, o solvente foi removido sob pressão reduzida, obtendo-se um óleo amarelo escuro.

Filtrou-se em uma coluna de sílica-gel, eluindo-se com hexano, obtendo-se um óleo ligeiramente amarelado. Esse óleo obtido foi destilado em um sistema de destilação horizontal $\left(1 \mathrm{mmHg} ; 50^{\circ} \mathrm{C}\right)$, obtendo-se 0,39g (65\%) de um óleo incolor, com $94 \%$ de pureza por cgl.

\section{Dados espectroscópicos}

$-\mathrm{RMN}{ }^{1} \mathrm{H}, 300 \mathrm{MHz}, \delta\left(\mathrm{CDCl}_{3}\right):$ 9,48 (s,1H); 2,59 (dd, 1H, Jab=13,7 e Jbd=1 Hz);

$$
\text { 2,27 (d,1H, Jcd=17,2Hz); 2,17 (dd, 1H, Jdc=17,2 e Jdb=1Hz); }
$$

$1,61(\mathrm{~d}, 1 \mathrm{H}, \mathrm{Jab}=13,7 \mathrm{~Hz}) ; 1,3(\mathrm{~s}, 3 \mathrm{H}) ; \quad 1,17(\mathrm{~s}, 3 \mathrm{H}) ; 1,03(\mathrm{~s}, 3 \mathrm{H})$.

$-\mathrm{RMN}{ }^{13} \mathrm{C}, 75 \mathrm{MHz}, \delta\left(\mathrm{CDCl}_{3}\right): 215,3 ; 198,7 ; 63,1 ; 53,2 ; 44,2 ; 33,8 ; 29,6 ; 28,9$;

\section{1,2 .}

-IV (Filme Líquido), $v_{\text {max }}: 2956 ; 2868 ; 2712 ; 1730 ; 1714 ; 1453 ; 1370 ; 1149 \mathrm{~cm}^{-1}$.

-EM, m / e, (abundância relativa, \%): $139(\mathbf{M}-15)^{+}(21) ; 126(12,8) ; 111(22,7)$;

$$
97(22,4) ; 83(100,0) ; 69(46,71) ; 55(40,04) .
$$




\section{V.3.3. PREPARAÇÀO}

DO

( \pm )-2-DIMETOXIMETIL-2,4,4-TRIMETIL-

CICLOPENTANONA (4).

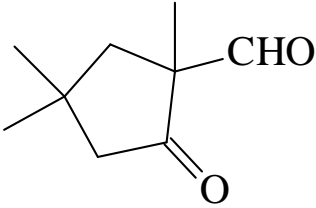

3

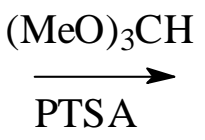

$77 \%$

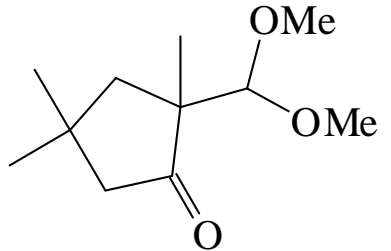

4

Dissolveu-se $(0,7946 \mathrm{~g} ; 5,16$ mmoles $)$ do composto 3 em 2,3 $\mathrm{mL}$ de $(\mathrm{MeO})_{3} \mathrm{CH}$ e em seguida adicionou-se um cristal de PTSA. . Deixou-se a mistura reacional sob agitação à temperatura ambiente por 4 horas. Neutralizou-se o meio com uma solução saturada de $\mathrm{K}_{2} \mathrm{CO}_{3}$ e em seguida secou-se a fase orgânica com sulfato de magnésio anidro. Após esse tempo o solvente foi removido sob pressão reduzida e o resíduo foi purificado por cromatografia de sílica-gel, eluindo-se com Hexano:Acetato, 9:1, obtendo-se 0,8045g (77\%) de um óleo amarelo.

\section{Dados espectroscópicos}

$-\mathrm{RMN}{ }^{1} \mathrm{H}, 300 \mathrm{MHz}, \delta\left(\mathrm{CDCl}_{3}\right)$ : 4,11 (s, 3H); 3,47 (s,3H); 3,40 (s, 3H); 2,33 (d, 1H,

$$
\begin{gathered}
\text { Jba=13,5 e Jbd=0,8Hz); 2,23 (dd, } \\
\text { 1H, Jdc=16,9 e Jca=0,8Hz); 2,10 (dd, } \\
\text { 1H, Jcd=16,9 e Jca=2Hz); 1,50 (dd, 1H, } \\
\text { Jab=13,5 e Jac=2Hz); 1,17 (s, 3H ); 1,16 } \\
(\mathrm{s}, 3 \mathrm{H}) ; 1,05(\mathrm{~s}, 3 \mathrm{H}) .
\end{gathered}
$$

$-\mathrm{RMN}{ }^{13} \mathrm{C}, 75 \mathrm{MHz}, \delta\left(\mathrm{CDCl}_{3}\right): \quad 221,2 ; 110,4 ; 58,1 ; 57,7 ; 55 ; 54,3 ; 44,4 ; 32,96 ; 30,2$;

30,$1 ; 22,2$.

-IV (Filme Líquido), $v_{\max }: 2956 ; 2868 ; 2832 ; 1734 ; 1454 ; 1190 ; 1100 \mathrm{~cm}^{-1}$.

-EM, m / e , (abundância relativa, \%): 169 (M-31) (16); 141 (17); 125 (7); 109 (17); 83

(39); 75 (100); 55 (31,5); 43 (47). 


\section{V.3.4.PREPARAÇÃO DOS COMPOSTOS (5a e 5b).}<smiles>COC(OC)C1(C)CC(C)(C)CC1=O</smiles>

4

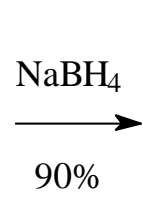<smiles>COC(OC)[C@]1(C)CC(C)(C)C[C@H]1O</smiles>

$5 \mathbf{a}$<smiles>COC(OC)[C@]1(C)CC(C)(C)CC1O</smiles>

$5 \mathbf{b}$

Dissolveu-se $(0,2513 \mathrm{~g} ; 1,25$ mmoles $)$ da cetona 4 em $10 \mathrm{~mL}$ de $\mathrm{MeOH}$. Manteve-se a solução a $0^{\circ} \mathrm{C}$ e em seguida adicionou-se $(0,0472 \mathrm{~g} ; 1,25$ mmoles $)$ de $\mathrm{NaBH}_{4}$. A reação foi mantida a $0^{\circ} \mathrm{C}$ sob constante agitação por 2 horas.

Após esse tempo, evaporou-se o $\mathrm{MeOH}$, adicionou-se água e extraiu-se várias vezes a fase aquosa com $\mathrm{Et}_{2} \mathrm{O}$. Lavou-se a fase orgânica com uma solução saturada de $\mathrm{NaCl}$, secou-se sob $\mathrm{MgSO}_{4}$ anidro e em seguida evaporou-se o solvente a pressão reduzida, obtendo-se 0,2284g (90\%) de um óleo amarelo, como uma mistura diastereoisomérica dos compostos cis $\mathbf{5 a}$ e trans $\mathbf{5} \mathbf{b}$, em uma proporção de 1:1.

A mistura foi separada por cromatografia em sílica-gel eluindo-se inicialmente com hexano e depois adicionando-se gradualmente acetato de etila até um máximo de $20 \%$ de acetato de etila em hexano. 


\section{Dados espectroscópicos}

(士) - CIS - 2 - DIMETOXIMETIL - 2,4,4 - TRIMETIL - CICLOPENTANOL (5a).<smiles>COC(OC)[C@]1(C)CC(C)(C)C[C@H]1O</smiles>

$5 a$

$-\mathrm{RMN}{ }^{1} \mathrm{H}, 300 \mathrm{MHz}, \delta\left(\mathrm{CDCl}_{3}\right): 4,27(\mathrm{~s}, 1 \mathrm{H}) ; 3,94(\mathrm{t}, 1 \mathrm{H}, \mathrm{Jec}=\mathrm{Jed}=5 \mathrm{~Hz}) ; 3,53$ $(\mathrm{s}, 3 \mathrm{H}) ; 3,51(\mathrm{~s}, 3 \mathrm{H}) ; 2,82(\mathrm{sl}, 1 \mathrm{H}, \mathrm{OH}) ; 1,89(\mathrm{~d}, 1 \mathrm{H}$,

$\mathrm{Jba}=13,3 \mathrm{~Hz}) ; 1,84$ (ddd,1H, Jcb=13,2; Jce=5 e Jca=1,3Hz); 1,57 (dd,1H, Jdc=13,2 e Jde=5Hz); 1,26 (dd,1H,Jab=13,3 e $\mathrm{Jac}=1,3 \mathrm{~Hz}) ; 1,13$ (s,3H);1,06 (s, 3H); 1,04 (s,3H).

$-\mathrm{RMN}{ }^{13} \mathrm{C}, 75 \mathrm{MHz}, \delta\left(\mathrm{CDCl}_{3}\right): 111,4 ; 81,6 ; 58,2 ; 57,7 ; 51,6 ; 49,0 ; 48,4 ; 36,2 ; 32,7$; 32,$3 ; 21,8$.

-IV (filme líquido), $v_{\text {max }}: 3465 ; 2954 ; 1463 ; 1376 ; 1186 ; 1071 \mathrm{~cm}^{-1}$.

--EM, m /e , (abundância, \%): $185\left(\mathbf{M}-\mathrm{H}_{2} \mathrm{O}\right)^{+}(5) ; 169$ (6,7); 153 (27); 121 (32); 95 (13); 75 (100); 59 (11); 41 (11). 
( \pm - TRANS - 2 - DIMETOXIMETIL - 2,4,4 - TRIMETIL - CICLOPENTANOL (5b).

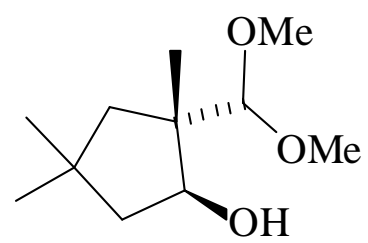

$\mathbf{5 b}$

$-\mathrm{RMN}{ }^{1} \mathrm{H}, 300 \mathrm{MHz}, \delta\left(\mathrm{CDCl}_{3}\right): 4.08(\mathrm{dd}, 1 \mathrm{H}, \mathrm{Jec}=11,3$ e Jed=6,92Hz); 3,93 (s, 1H); $3,45(\mathrm{~s}, 3 \mathrm{H}) ; 3,43(\mathrm{~s}, 3 \mathrm{H}) ; 2,52(\mathrm{sl}, 1 \mathrm{H}, \mathrm{OH}) ; 1,68$ $(\mathrm{dd}, 1 \mathrm{H}, \mathrm{Jdc}=12,2$ e Jde=6,9); 1,57 (t ,1H, Jcd=12,2 e Jce $=11,3 \mathrm{~Hz}) ; 1,50$ (d, 1H, Jba=13,6Hz); 1,28 (d, $1 \mathrm{H}, \mathrm{Jab}=13,6 \mathrm{~Hz}) ; 1,02(\mathrm{~s}, 3 \mathrm{H}) ; 0,94(\mathrm{~s}, 3 \mathrm{H}) ; 0,93$ $(\mathrm{s}, 3 \mathrm{H})$.

$-\mathrm{RMN}{ }^{13} \mathrm{C}, 75 \mathrm{MHz}, \delta\left(\mathrm{CDCl}_{3}\right): 114,2 ; 75,1 ; 58,7 ; 57,5 ; 50,3 ; 48,7 ; 46 ; 36,1 ; 32,5$; 32,$4 ; 15,6$.

-IV (filme líquido), $v_{\max }: 3422 ; 2950 ; 2865 ; 1463 ; 1364 ; 1188 ; 1106 ; 1072 \mathrm{~cm}^{-1}$.

-EM, m / e, (abundância,\%): $185\left(\text { M- } \mathrm{H}_{2} \mathrm{O}\right)^{+}(3,3) ; 169$ (9,4); 153 (32); 121 (26); 95 (11,3); 75 (100); 41 (11). 


\section{V.3.5.PREPARAÇÃO DO ( $( \pm)$ - CIS- 1-DIMETOXIMETIL- 2- ACETOXI-1,4,4- TRIMETIL- CICLOPENTANO (6a).}<smiles>COC(OC)[C@]1(C)CC(C)(C)C[C@H]1O</smiles>

$5 \mathbf{a}$

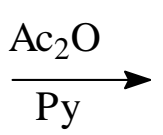

$81 \%$<smiles>COC(OC)[C@]1(C)CC(C)(C)C[C@@H]1OC(C)=O</smiles>

6a

Dissolveu-se o composto 5a (0,3429g; 1,7 mmoles) em $2 \mathrm{~mL}$ de piridina seca e em seguida adicionou-se $2 \mathrm{~mL}$ de anidrido acético. Manteve-se a mistura reacional sob agitação durante a noite à temp. ambiente. Após esse tempo resfriou-se o balão a $0^{\circ} \mathrm{C}$ e adicionou-se $2 \mathrm{~mL}$ de $\mathrm{MeOH}$ gota a gota.

Diluiu-se com $10 \mathrm{~mL}$ de $\mathrm{Et}_{2} \mathrm{O}$ e lavou-se várias vezes com uma solução de $\mathrm{CuSO}_{4}$ a $20 \%$, secou-se a fase orgânica com $\mathrm{MgSO}_{4}$ anidro, o solvente foi removido sob pressão reduzida, obtendo-se um óleo incolor que foi purificado por cromatografia em sílica-gel, eluindo-se com Hexano: AcOEt, 8:2, obtendo-se 0,3359g (81\%) do acetato-acetal 6a.

\section{Dados espectroscópicos}

$-\mathrm{RMN}{ }^{1} \mathrm{H}, 300 \mathrm{MHz}, \delta\left(\mathrm{CDCl}_{3}\right): 4.91(\mathrm{dd}, 1 \mathrm{H}, \mathrm{Jed}=4,8$ e Jec=2,5Hz); 4,21 (s, 1H);

$3,51(\mathrm{~s}, 3 \mathrm{H}) ; 3,43$ (s, 3H); 2,05 (s, 3H); 1,93 (dd, 1H, $\mathrm{Jcd}=14,5 ; \mathrm{Jce}=4,8$ e Jca=1,3Hz); $1,81(\mathrm{~d}, 1 \mathrm{H}, \mathrm{Jba}$ $13,6 \mathrm{~Hz}) ; 1,63(\mathrm{dd}, 1 \mathrm{H}, \mathrm{Jdc}=14,5$ e $\mathrm{Jde}=2,5 \mathrm{~Hz}) ; 1,39$ $(\mathrm{dd}, 1 \mathrm{H}, \mathrm{Jab}=13,6$ e Jac=1,3Hz); 1,10 (s, 3H); 1,09 $(\mathrm{s}, 3 \mathrm{H}) ; 1,05$ (s, 3H).

$-\mathrm{RMN}{ }^{13} \mathrm{C}, 75 \mathrm{MHz}, \delta\left(\mathrm{CDCl}_{3}\right): 169,9 ; 110,8 ; 83,8 ; 58,3 ; 57,8 ; 51,9 ; 49,2 ; 45,9 ; 36,8$; 33,$1 ; 32,6 ; 21,3 ; 19,4$.

-IV (filme líquido), $v_{\max }: 1737 ; 1366 ; 1242 ; 1192 ; 1102 ; 1069 ; 1027 \mathrm{~cm}^{-1}$.

-EM, m / e, (abundância,\%): $213(\mathbf{M}-31)^{+}(24,48) ; 153$ (45,4); 121 (18,6); 95 (18); 75 (100); 43 (23). 


\section{V.3.6.PREPARAÇÃO DO ( $( \pm)$ - TRANS - 1-DIMETOXIMETIL- 2- ACETOXI- 1,4,4- TRIMETIL- CICLOPENTANO (6b).}<smiles>COC(OC)[C@]1(C)CC(C)(C)CC1O</smiles>

$\mathbf{5 b}$

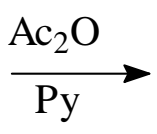

$79 \%$<smiles>COC(OC)[C@]1(C)CC(C)(C)C[C@@H]1OC</smiles>

6b

Dissolveu-se o composto 5b (0,3429g; 1,7 mmoles) em $2 \mathrm{~mL}$ de piridina seca e em seguida adicionou-se $2 \mathrm{~mL}$ de anidrido acético. Manteve-se a mistura reacional sob agitação durante a noite à temp. ambiente. Após esse tempo resfriou-se o balão a $0^{\circ} \mathrm{C}$ e adicionou-se $2 \mathrm{~mL}$ de $\mathrm{MeOH}$ gota a gota.

Diluiu-se com $10 \mathrm{~mL}$ de $\mathrm{Et}_{2} \mathrm{O}$ e lavou-se várias vezes com uma solução de $\mathrm{CuSO}_{4}$ a $20 \%$, secou-se a fase orgânica com $\mathrm{MgSO}_{4}$ anidro, o solvente foi removido sob pressão reduzida, obtendo-se um óleo incolor que foi purificado por cromatografia em sílica-gel, eluindo-se com Hexano: AcOEt, 8:2, obtendo-se 0,2916g (79\%) do acetato-acetal $\mathbf{6 b}$.

\section{Dados espectroscópicos}

$-\mathrm{RMN}{ }^{1} \mathrm{H}, 300 \mathrm{MHz}, \delta\left(\mathrm{CDCl}_{3}\right): 5,2(\mathrm{dd}, 1 \mathrm{H}, \mathrm{Jec}=9,3$ e Jed=6,9Hz); 3,99 (s, 1H);

$3,52(\mathrm{~s}, 3 \mathrm{H}) ; 3,44(\mathrm{~s}, 3 \mathrm{H}) ; 2,02(\mathrm{~s}, 3 \mathrm{H}) ; 1,83$

(ddd, 1H, Jdc=12,4; Jde=6,8 e Jdb=1,3Hz); 1,81 (dd ,1H, Jba=13,7 e Jbd=1,3 Hz); 1,61 (ddd, 1H, Jcd=12,4 ; Jce $=9,2$ e Jca $=0,5 H z) ; 1,27$ (dd, 1H, $\mathrm{Jab}=13,7$ e Jac $=0,5 \mathrm{~Hz}) ; 1,08(\mathrm{~s}, 3 \mathrm{H}) ; 1,02(\mathrm{~s}, 3 \mathrm{H})$; $1,01(\mathrm{~s}, 3 \mathrm{H})$.

$-\mathrm{RMN}{ }^{13} \mathrm{C}, 75 \mathrm{MHz}, \delta\left(\mathrm{CDCl}_{3}\right): 170,6 ; 11,5 ; 77,9 ; 58,3 ; 57,7 ; 50,8 ; 47,4 ; 44,8 ; 34,3$; 31,$8 ; 31,2 ; 21,1 ; 18,4$.

-IV (filme líquido), $v_{\max }: 1742 ; 1381 ; 1187 ; 1117 ; 1072 ; 1030 \mathrm{~cm}^{-1}$

-EM, m / e, (abundância,\%): $213\left(\right.$ M-31) ${ }^{+}(24,48) ; 153$ (45,4); 121 (18,6); 95 (18); 75 (100); 43 (23). 


\section{V.3.7. PREPARAÇÃO DO $( \pm)$ - CIS - 2-ACETOXI - 1,4,4 - TRIMETIL- CICLOPENTANO-1-CARBOXALDEÍDO (7a)}<smiles>COC(OC)[C@]1(C)CC(C)(C)C[C@H]1OC(C)=O</smiles>

6a<smiles>CC(C)(C)[O+]S(=O)(=O)O</smiles>

$88 \%$<smiles>CC(=O)O[C@@H]1CC(C)(C)C[C@]1(C)C=O</smiles>

$7 \mathbf{a}$

Adicionou-se $315 \mu \mathrm{L}$ de uma solução aquosa de ácido oxálico a 15\% a uma suspensão de 3,089g de sílica-gel em $8 \mathrm{~mL}$ de $\mathrm{CH}_{2} \mathrm{Cl}_{2}$.

Agitou-se por 10 minutos e depois adicionou-se $(0,3429 \mathrm{~g} ; 1,40 \mathrm{mmoles})$ do composto $\mathbf{6 a}$ em $1 \mathrm{~mL}$ de de $\mathrm{CH}_{2} \mathrm{Cl}_{2}$. A mistura foi mantida sob agitação durante 19 horas à temperatura ambiente, após esse tempo adicionou-se 0,2000 g de $\mathrm{NaHCO}_{3 \text { (pó) }}$ e agitou-se por 5 minutos. Depois filtrou-se a vácuo, lavando-se o sólido várias vezes com $\mathrm{CH}_{2} \mathrm{Cl}_{2}$. Secou-se o solvente com $\mathrm{MgSO}_{4}$ anidro, evaporou-se e obteve-se $0,2473 \mathrm{~g}(88 \%)$ de um óleo ligeiramente amarelo.

\section{Dados espectroscópicos}

$-\mathrm{RMN}{ }^{1} \mathrm{H}, 300 \mathrm{MHz}, \delta\left(\mathrm{CDCl}_{3}\right): 9,64(\mathrm{~s}, 1 \mathrm{H}) ; 5,15(\mathrm{t}, 1 \mathrm{H}, \mathrm{Jec}=\mathrm{Jed}=6,5 \mathrm{~Hz}) ; 2,24(\mathrm{~d}$,

$1 \mathrm{H}, \quad \mathrm{Jba}=13,9 \mathrm{~Hz}) ; 2,03(\mathrm{~s}, 3 \mathrm{H}) ; 2,03(\mathrm{ddd}, 1 \mathrm{H}$, $\mathrm{Jcd}=13,5 ; \quad \mathrm{Jce}=6,5$ e Jca=2,6 Hz); 1,67 (dd, $1 \mathrm{H}$, $\mathrm{Jdc}=13,5$ e $\mathrm{Jde}=6,5 \mathrm{~Hz}) ; 1,32(\mathrm{dd}, 1 \mathrm{H}, \mathrm{Jab}=13,9$ e $\mathrm{Jac}=2,6 \mathrm{~Hz}) ; 1,24$ (s, 3H); 1,15 (s, 3H); 1,13 (s, 3H).

$-\mathrm{RMN}{ }^{13} \mathrm{C}, 75 \mathrm{MHz}, \delta\left(\mathrm{CDCl}_{3}\right): 202,5 ; 170,1 ; 82,7 ; 56,3 ; 46,3 ; 45,8 ; 36,7 ; 31,4$;

$$
31 ; 20,8 ; 20,8 \text {. }
$$

-IV (filme líquido), $v_{\max }: 2951 ; 2861 ; 1739 ; 1708 ; 1463 ; 1370 ; 1238 ; 1144 ; 1071 \mathrm{~cm}^{-1}$.

-EM, m / e, (abundância,\%): 197 (M-1) ${ }^{+}(3,74) ; 167$ (11,20); 149 (33,59); $126(37,23)$;

$$
83 \text { (46,25); } 43 \text { (100); 17,8 (42,61). }
$$




\section{V.3.8. PREPARAÇÃO DO ( \pm ) - TRANS - 2-ACETOXI - 1,4,4 - TRIMETIL- CICLOPENTANO-1-CARBOXALDEÍDO (7b)}<smiles>COC(OC)[C@]1(C)CC(C)(C)CC1OC(C)=O</smiles>

6b

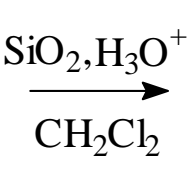

$84 \%$

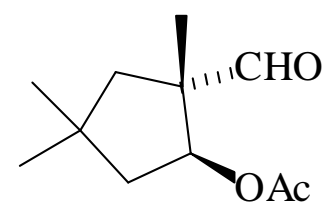

$7 \mathbf{b}$

Adicionou-se $315 \mu \mathrm{L}$ de uma solução aquosa de ácido oxálico a $15 \%$ a uma suspensão de 3,089g de sílica-gel em $8 \mathrm{~mL}$ de $\mathrm{CH}_{2} \mathrm{Cl}_{2}$.

Agitou-se por 10 minutos e depois adicionou-se $(0,3429 \mathrm{~g} ; 1,40 \mathrm{mmoles})$ do composto $\mathbf{6 b}$ em $1 \mathrm{~mL}$ de $\mathrm{CH}_{2} \mathrm{Cl}_{2}$. A mistura foi mantida sob agitação durante 19 horas a temperatura ambiente, após esse tempo adicionou-se 0,2000 g de $\mathrm{NaHCO}_{3 \text { (pó) }} \mathrm{e}$ agitou-se por 5 minutos. Depois filtrou-se a vácuo, lavando-se o sólido várias vezes com $\mathrm{CH}_{2} \mathrm{Cl}_{2}$. Secou-se o solvente com $\mathrm{MgSO}_{4}$ anidro, evaporou-se e obteve-se 0,2460g (84\%) de um óleo ligeiramente amarelo.

\section{Dados espectroscópicos}

$-\mathrm{RMN}{ }^{1} \mathrm{H}, 300 \mathrm{MHz}, \delta\left(\mathrm{CDCl}_{3}\right): 9,52(\mathrm{~s}, 1 \mathrm{H}) ; 5,31(\mathrm{dd}, 1 \mathrm{H}, \mathrm{Jec}=8,7$ e Jed=6,5Hz);

2,04 (s, 3H); 1,97 (dd, 1H, Jba=13,9 e Jbd=1,2Hz );

$1,93(\mathrm{ddd}, 1 \mathrm{H}, \mathrm{Jdc}=13 ; \mathrm{Jde}=6,5 \mathrm{e} \mathrm{Jdb}=1,2 \mathrm{~Hz}) ; 1,78$

$(\mathrm{dd}, 1 \mathrm{H}, \mathrm{Jcd}=13$ e Jce=8,7 Hz); $1,45(\mathrm{~d}, 1 \mathrm{H}, \mathrm{Jab}=$ $13,9 \mathrm{~Hz}) ; 1,08$ (s, 3H); 1,02 (s, 3H); 1,01 (s, 3H).

$-\mathrm{RMN}{ }^{13} \mathrm{C}, 75 \mathrm{MHz}, \delta\left(\mathrm{CDCl}_{3}\right): 200,4 ; 170,3 ; 76,39 ; 56,45 ; 47,93 ; 44,94 ; 35,15 ; 31,18 ;$ 30,$3 ; 20,58 ; 15,9$.

-IV (filme líquido), $v_{\max }: 2955 ; 2873 ; 1741 ; 1705 ; 1464 ; 1377 ; 1254 \mathrm{~cm}^{-1}$.

-EM, m / e, (abundância,\%): $197\left(\right.$ M-1) ${ }^{+}(5,4) ; 153$ (8,97); $126(38,7) ; 95$ (24,23); 83 $(46,10) ; 69(40,43) ; 55(16,87) ; 43(100)$. 


\section{V.3.9. PREPARAÇÃO DO ( $( \pm)$-CIS - 2-ACETOXI - 1,4,4 - TRIMETIL- CICLOPENTANO-1- ÁCIDO CARBOXÍLICO (8a)}<smiles>CC(=O)O[C@H]1CC(C)(C)C[C@]1(C)C=O</smiles>

7a

$$
\underset{88 \%}{\stackrel{\mathrm{NaClO}_{2}}{\underset{\mathrm{pH} 4,5}{\longrightarrow}}}
$$<smiles>CC(=O)O[C@H]1CC(C)(C)C[C@]1(C)C(=O)O</smiles>

$8 \mathbf{a}$

Dissolveu-se $\mathrm{NaH}_{2} \mathrm{PO}_{4} \cdot \mathrm{H}_{2} \mathrm{O}(0,2865 \mathrm{~g}$ em $2 \mathrm{~mL}$ de água), $\mathrm{pH}=4,5$; e em seguida adicionou-se uma solução de $\mathrm{NaClO}_{2}$ a $30 \%(0,86 \mathrm{~mL})$.

Gotejou-se essa mistura a um balão contendo uma solução do composto 7a $(0,0530 \mathrm{~g})$ em $1,2 \mathrm{~mL}$ de t-BuOH e $0,36 \mathrm{~mL}$ de 2-metil-2-buteno. A reação permaneceu sob forte agitação à temperatura ambiente durante 3 horas. Após esse tempo acertou-se o pH=10 com uma solução de $\mathrm{NaOH}$ 6M. Lavou-se duas vezes a fase aquosa com hexano.

Em seguida, a fase aquosa foi acidificada com $\mathrm{HCl}$ até $\mathrm{pH}=3,5$ e extraida com $\mathrm{Et}_{2} \mathrm{O}$. Secou-se sob $\mathrm{MgSO}_{4}$ anidro e evaporou-se o solvente, obtendo-se $0,0504 \mathrm{~g}$ $(88 \%)$ de um óleo incolor .

\section{Dados espectroscópicos}

-RMN ${ }^{1} \mathrm{H}, 300 \mathrm{MHz}, \delta\left(\mathrm{CDCl}_{3}\right): 10,21(\mathrm{sl}, 1 \mathrm{H}, \mathrm{OH}) ; 5,17(\mathrm{t}, 1 \mathrm{H}, \mathrm{Jec}=\mathrm{Jed}=5,2 \mathrm{~Hz})$;

2,41 (d, 1H, Jba=14 Hz); 1,96 (ddd, 1H, Jcd=13,8;

$\mathrm{Jce}=5,2$ e Jca=0,6 Hz); 2,01(s, 3H); 1,83 (dd, 1H,

$\mathrm{Jdc}=13,8 ; \mathrm{Jde}=5,2 \mathrm{~Hz}) ; 1,49(\mathrm{dd}, 1 \mathrm{H}, \mathrm{Jab}=14 \mathrm{e} \mathrm{Jac}=$

$0,6 \mathrm{~Hz}) ; 1,36(\mathrm{~s}, 3 \mathrm{H}) ; 1,14(\mathrm{~s}, 3 \mathrm{H}) ; 1,13(\mathrm{~s}, 3 \mathrm{H})$.

$-\mathrm{RMN}{ }^{13} \mathrm{C}, 75 \mathrm{MHz}, \delta\left(\mathrm{CDCl}_{3}\right): 181,28 ; 170,29 ; 82,83 ; 54,05 ; 48,68 ; 45,14 ; 36,67$;

32,$21 ; 31,92 ; 24,36 ; 21,14$.

-IV (filme líquido), $v_{\max }: 3306-2572 ; 2917 ; 1743 ; 1709 ; 1464 ; 1375 ; 1248 \mathrm{~cm}^{-1}$.

-EM, m / e, (abundância,\%): 154 (M-60)+(25); 153 (6); 126 (64); 109 (23); 83 (55);

$$
69(61) ; 43 \text { (100). }
$$




\section{V.3.10. PREPARAÇÃO DO ( \pm -TRANS-2-ACETOXI-1,4,4- TRIMETIL- CICLOPENTANO-1- ÁCIDO CARBOXÍLICO (8b)}

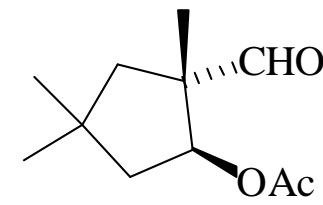

7b

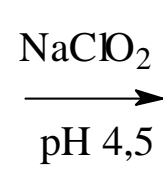

$91 \%$

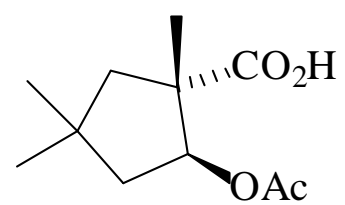

8b

Dissolveu-se $\mathrm{NaH}_{2} \mathrm{PO}_{4} \cdot \mathrm{H}_{2} \mathrm{O}(0,2865 \mathrm{~g}$ em $2 \mathrm{~mL}$ de água), $\mathrm{pH}=4,5$; e em seguida adicionou-se uma solução de $\mathrm{NaClO}_{2}$ a $30 \%(0,86 \mathrm{~mL})$.

Gotejou-se essa mistura a um balão contendo uma solução do composto 7b $(0,0530 \mathrm{~g})$ em 1,2 mL de t-BuOH e 0,36 mL de 2-metil-2-buteno. A reação permaneceu sob forte agitação à temperatura ambiente durante 3 horas. Após esse tempo acertou-se o pH=10 com uma solução de $\mathrm{NaOH} 6 \mathrm{M}$. Lavou-se duas vezes a fase aquosa com hexano.

Em seguida, a fase aquosa foi acidificada com $\mathrm{HCl}$ até $\mathrm{pH}=3,5$ e extraida com $\mathrm{Et}_{2} \mathrm{O}$. Secou-se sob $\mathrm{MgSO}_{4}$ anidro e evaporou-se o solvente, obtendo-se $0,0511 \mathrm{~g}$ (91\%) de um óleo incolor .

\section{Dados espectroscópicos}

$-\mathrm{RMN}^{1} \mathrm{H}, 300 \mathrm{MHz}, \delta\left(\mathrm{CDCl}_{3}\right): 10,25(\mathrm{sl}, 1 \mathrm{H}, \mathrm{OH}) ;$ 5,54(dd, 1H, Jec=8,1 e Jed=6,6

$\mathrm{Hz}$ ); 2,17 (dd, 1H, Jba=13,6 e Jbd=2,6 Hz); 2,05(s,

$3 \mathrm{H}) ; 1,99$ (ddd, 1H, Jdc=13,1; Jde=6,6 e Jdb=2,6 Hz);

$1,66(\mathrm{dd}, 1 \mathrm{H}, \mathrm{Jcd}=13,1$ e $\mathrm{Jce}=8,1 \mathrm{~Hz}) ; 1,59(\mathrm{~d}, 1 \mathrm{H}$,

$\mathrm{Jab}=13,6 \mathrm{~Hz})$.

-RMN ${ }^{13} \mathrm{C}, 75 \mathrm{MHz}, \delta\left(\mathrm{CDCl}_{3}\right): 182,88 ; 170,69 ; 78,69 ; 52,12 ; 51,24 ; 45,20 ; 35,48$;

31,$63 ; 30,67 ; 20,94 ; 19,77$.

-IV (filme líquido), $v_{\max }: 3351-2542 ; 2962 ; 1743 ; 1701 ; 1468 ; 1371 ; 1244 \mathrm{~cm}^{-1}$

-EM, m / e, (abundância,\%): 169 (6); 154 (28); 153 (14); 139 (14); 126 (70); 111

(21); 83 (69); 69 (69); 43 (100). 
V.3.11. PREPARAÇÃO DO ( \pm -CIS-2-ACETOXI-1,4,4-TRIMETILCICLOPENTANO-1-CARBOXILATO DE METILA (9a)<smiles>CC(=O)O[C@H]1CC(C)(C)C[C@]1(C)C(=O)O</smiles>

8a

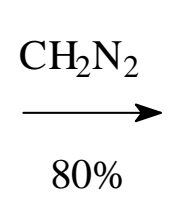

$80 \%$<smiles>CC(=O)O[C@H]1CC(C)(C)C[C@]1(C)C(C)=O</smiles>

9a

Resfriou-se uma solução de 2,14g de Diazald com gelo e em seguida adicionou-se uma solução de KOH em 10 mL de EtOH 96\%.

Depois de 5 minutos destilou-se a solução de $\mathrm{CH}_{2} \mathrm{~N}_{2}$ em $\mathrm{Et}_{2} \mathrm{O}$. Dissolveu-se o ácido 8a $0,1550 \mathrm{~g}$ em $3 \mathrm{~mL}$ de $\mathrm{Et}_{2} \mathrm{O}$ e em seguida adicionou-se lentamente a solução etérea de $\mathrm{CH}_{2} \mathrm{~N}_{2}$ até a cor amarela não desaparecer. Evaporou-se o solvente e obteve-se $0,1320 \mathrm{~g}(80 \%)$ de um óleo ligeiramente amarelo.

\section{Dados espectroscópicos}

$-\mathrm{RMN}{ }^{1} \mathrm{H}, 300 \mathrm{MHz}, \delta\left(\mathrm{CDCl}_{3}\right): 5,13(\mathrm{t}, 1 \mathrm{H}, \mathrm{Jec}=\mathrm{Jed}=5 \mathrm{~Hz}) ; 3,67(\mathrm{~s}, 3 \mathrm{H}) ; 2,42(\mathrm{~d}, 1 \mathrm{H}$, $\mathrm{Jba}=13,8 \mathrm{~Hz}) ; 1,98$ (s, 3H); 1,95 (ddd, 1H, Jcd=14; Jce $=5$ e Jca=0,7 Hz); $1,78 \quad(\mathrm{dd}, \quad 1 \mathrm{H}, \quad \mathrm{Jdc}=14$ e $\mathrm{Jde}=5 \mathrm{~Hz}) ; 1,47(\mathrm{dd}, 1 \mathrm{H}, \mathrm{Jab}=13,8$ e $\mathrm{Jac}=0,7 \mathrm{~Hz})$; $1,36(\mathrm{~s}, 3 \mathrm{H}) ; 1,14(\mathrm{~s}, 3 \mathrm{H}) ; 1,13(\mathrm{~s}, 3 \mathrm{H})$.

$-\mathrm{RMN}{ }^{13} \mathrm{C}, 75 \mathrm{MHz}, \delta\left(\mathrm{CDCl}_{3}\right): 175,35 ; 170,07 ; 83,04 ; 54,26 ; 51,75 ; 48,82 ; 45,26$;

$$
36,71 ; 32,27 ; 32,07 ; 24,41 ; 21,12 \text {. }
$$

-IV (filme líquido), $v_{\text {max }}: 2947 ; 2887 ; 1743 ; 1464 ; 1375 ; 1240 \mathrm{~cm}^{-1}$.

-EM, m / e, (abundância,\%): $186(\text { M-42) })^{+}(6) ; 169$ (4); 154 (8); 153 (10); 128 (15); 109

$$
\text { (32); } 101 \text { (100); } 93 \text { (12); } 68 \text { (22); } 43 \text { (45). }
$$




\section{V.3.12. PREPARAÇÃO DO (士)-TRANS-2-ACETOXI-1,4,4-TRIMETIL- CICLOPENTANO-1-CARBOXILATO DE METILA (9b)}

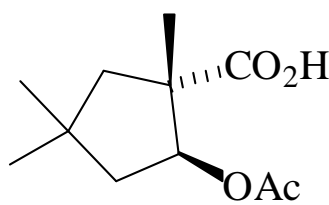

8b

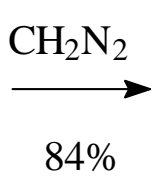

$84 \%$

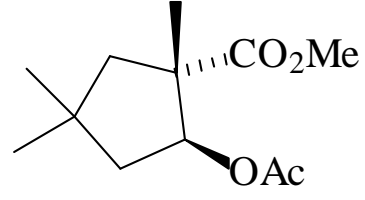

9b

Resfriou-se uma solução de $2,14 \mathrm{~g}$ de Diazald com gelo e em seguida adicionou-se uma solução de KOH em 10 mL de EtOH 96\%.

Depois de 5 minutos destilou-se a solução de $\mathrm{CH}_{2} \mathrm{~N}_{2}$ em $\mathrm{Et}_{2} \mathrm{O}$. Dissolveu-se o ácido $8 \mathbf{b} \quad 0,1550 \mathrm{~g}$ em $3 \mathrm{~mL}$ de $\mathrm{Et}_{2} \mathrm{O}$ e em seguida adicionou-se lentamente a solução etérea de $\mathrm{CH}_{2} \mathrm{~N}_{2}$ até a cor amarela não desaparecer. Evaporou-se o solvente e obteve-se $0,1386 \mathrm{~g}(84 \%)$ de um óleo ligeiramente amarelo.

\section{Dados espectroscópicos}

$-\mathrm{RMN}{ }^{1} \mathrm{H}, 300 \mathrm{MHz}, \delta\left(\mathrm{CDCl}_{3}\right):$ 5,53(dd, $1 \mathrm{H}, \mathrm{Jec}=7,6$ e Jed=6,3 Hz); 3,69 (s, 3H); 2,14

(dd, 1H, Jba=13,7 e Jbd=1,4 Hz); 2,04(s, 3H); 1,94

(ddd, 1H, Jdc=13,2; Jde=6,3 e Jdb=1,4 Hz); 1,65 (dd,

$1 \mathrm{H}, \mathrm{Jcd}=13,2 ; \mathrm{Jce}=7,6 \mathrm{~Hz}) ; 1,56(\mathrm{~d}, 1 \mathrm{H}, \mathrm{Jab}=13,7$

$\mathrm{Hz}) ; 1,21$ (s, 3H); 1,11 (s, 3H); 1,05 (s, 3H).

$-\mathrm{RMN}{ }^{13} \mathrm{C}, 75 \mathrm{MHz}, \delta\left(\mathrm{CDCl}_{3}\right): 177,08 ; 170,29 ; 78,87 ; 52,41 ; 52,11 ; 51,10 ; 45,25$;

$$
35,43 ; 31,81 ; 30,83 ; 20,97 ; 19,83 \text {. }
$$

-IV (filme líquido), $v_{\text {max }}: 2962 ; 2872 ; 1734 ; 1464 ; 1375 ; 1244 \mathrm{~cm}^{-1}$.

-EM, m / e, (abundância,\%): $197\left(\right.$ M-31) ${ }^{+}(2) ; 186$ (5); 168 (11); 153 (13); 128

$$
\text { (12); } 109 \text { (24); } 101 \text { (100); } 83 \text { (12); } 68 \text { (17); } 43 \text { (41). }
$$




\section{V.3.13. PREPARAÇÃO DOS COMPOSTOS (10a e 10b)}

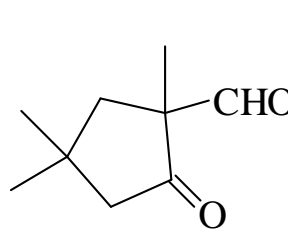

3

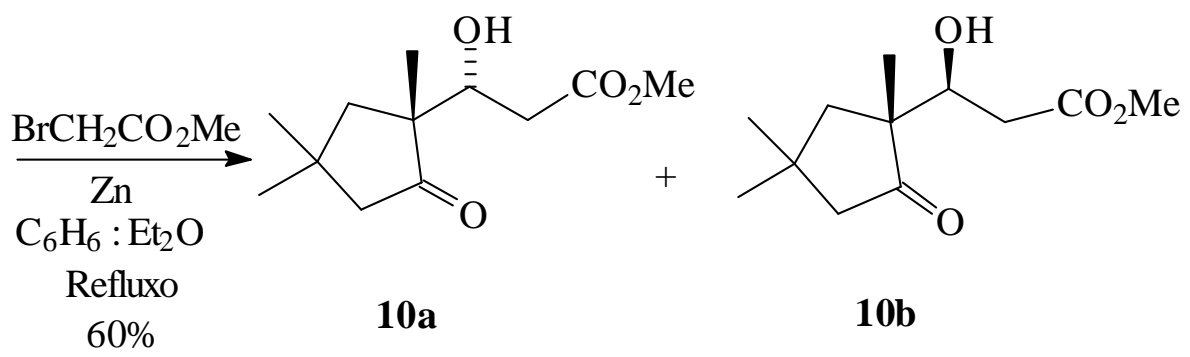

A um balão de $15 \mathrm{~mL}$ seco em estufa acoplado com condensador de refluxo e sob atmosfera de $\mathrm{N}_{2}$, adicionou-se $(0,1864 \mathrm{~g} ; 2,85$ mmoles $)$ de zinco em pó ativo.

Em seguida, adicionou-se 1,0 $\mathrm{mL}$ de uma solução de $(0,4389 \mathrm{~g} ; 2,85$ mmoles) do ceto-aldeído 3; (0,3555g ( 0,22 mL; 2,32 mmoles) do Bromoacetato de

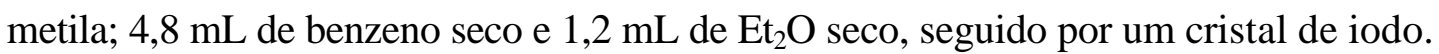

Agitou-se magneticamente sob aquecimento; logo que começou a reação adicionou-se aos poucos o restante da solução. (A adição foi feita em 20 minutos). Em seguida, manteve-se sob refluxo por 1 hora.

Esfriou-se a mistura reacional e manteve-se o balão a $0^{\circ} \mathrm{C}$ e adicionou-se $5 \mathrm{~mL}$ de $\mathrm{H}_{2} \mathrm{SO}_{4}$ a $10 \%$ e agitou-se vigorosamente por 5 minutos. Separou-se as fases, e a fase aquosa foi extraída várias vezes com $\mathrm{Et}_{2} \mathrm{O}$. Juntou-se as fases orgânicas e lavouse 2 vezes com $\mathrm{H}_{2} \mathrm{SO}_{4}$ a $5 \%$ e com uma solução de $\mathrm{Na}_{2} \mathrm{CO}_{3}$ a $5 \%$. Lavou-se novamente com $\mathrm{H}_{2} \mathrm{SO}_{4} 5 \%$ e 2 vezes com água. Secou-se a fase orgânica sob $\mathrm{MgSO}_{4}$ anidro, evaporou-se e obteve-se 0,3890g (60\%) de um óleo, como uma mistura diastereoisomérica dos compostos 10a e 10b, em uma proporção de 2:1.

A mistura foi separada por cromatografia em sílica-gel eluindo-se inicialmente com hexano e depois adicionando-se gradualmente acetato de etila até um máximo de $20 \%$ de acetato de etila em hexano. 


\section{Dados espectroscópicos}

rel-[3 $\alpha, 1^{\prime} \beta$ ]-( \pm$)$ - 3 -Hidroxi-3-(1',4',4'-trimetil-2'-oxo-ciclopentil)-propanoato de metila (10a).

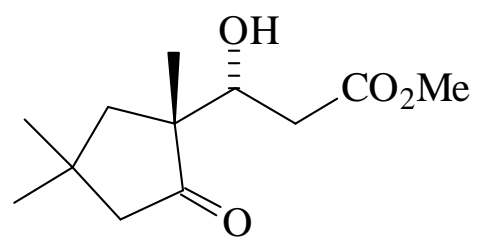

$10 a$

$-\mathrm{RMN}{ }^{1} \mathrm{H}, 300 \mathrm{MHz}, \delta\left(\mathrm{CDCl}_{3}\right): 3,96(\mathrm{dt}, 1 \mathrm{H}, \mathrm{Jeg}=10,2$; Jef=Je-OH=2,1 Hz);

3,71(s,3H); 3,06 (d, 1H, OH, JOH-e=2,1Hz); 2,56

(dd, 1H, Jgf=16 e Jge=10,2 Hz); 2,39 (dd, 1H, Jfg=16

e Jfe $=2,1 \mathrm{~Hz}) ; 2,31$ (d, 1H, Jdc=17,4 Hz); 2,23 (dd, 1H,

$\mathrm{Jcd}=17,4$ e Jca=1,2 Hz); 2,02 (d, 1H, Jba=13,2 Hz);

1,64 (dd, 1H, Jab= 13,2 e Jac=1,2Hz); 1,17 (s, 3H);

$1,16(\mathrm{~s}, 3 \mathrm{H}) ; 1,11(\mathrm{~s}, 3 \mathrm{H})$.

$-\mathrm{RMN}{ }^{13} \mathrm{C}, 75 \mathrm{MHz}, \delta\left(\mathrm{CDCl}_{3}\right): 223,4 ; 173,1 ; 72,8 ; 54,1 ; 51,89 ; 51,8 ; 48,3 ; 36,1 ; 33,1$;

30,$5 ; 30,1 ; 20,2$.

-IV (filme líquido), $v_{\max }: 3499 ; 2955 ; 2869 ; 1731 ; 1440 ; 1370 ; 1304 ; 1266 ; 1172 ; 1036$ $\mathrm{cm}^{-1}$.

--EM, m /e , (abundância, \%): $210\left(\text { M- } \mathrm{H}_{2} \mathrm{O}\right)^{+}$(9); 178 (13); 163 (14); 126 (100); 111

(84); 83 (53); 69 (50); 43 (52). 


\section{Dados espectroscópicos}

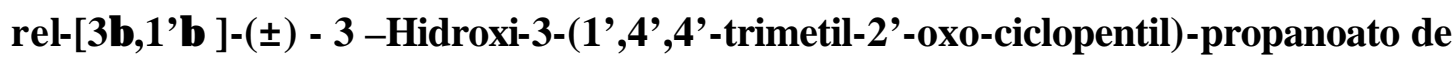
metila (10b).<smiles>CC(=O)C[C@H](O)[C@]1(C)CC(C)(C)CC1=O</smiles>

10b

$-\mathrm{RMN}{ }^{1} \mathrm{H}, 300 \mathrm{MHz}, \delta\left(\mathrm{CDCl}_{3}\right): 4,07$ (ddd, 1H, Jef=10,7; Je-OH=3,1 e Jeg=2,3);

$3,71(\mathrm{~s}, 3 \mathrm{H}) ; 3,05(\mathrm{~d}, 1 \mathrm{H}, \mathrm{OH}, \mathrm{JOH}-\mathrm{e}=3,1 \mathrm{~Hz}) ; 2,54$

$(\mathrm{dd}, 1 \mathrm{H}, \mathrm{Jgf}=16,5$ e Jeg=2,3Hz); 2,32 (dd, 1H,

$\mathrm{Jfg}=16,5$ e Jfe=10,7Hz); 2,27 (d, 1H, Jdc=17,3Hz);

2,24 (d, 1H, Jba=13,2 Hz); 2,19 (dd, 1H, Jcd=17,3 e

$\mathrm{Jca}=1,4 \mathrm{~Hz}) ; 1,5$ (dd, 1H, Jab= 13,2 e Jac

=1,4 Hz);1,19 (s, 3H); 1,08 (s, 3H); 1,06 (s, 3H).

$-\mathrm{RMN}{ }^{13} \mathrm{C}, 75 \mathrm{MHz}, \delta\left(\mathrm{CDCl}_{3}\right): 222,3 ; 173,3 ; 72,16 ; 54,4 ; 53,2 ; 51,9 ; 44,6 ; 36,1 ; 32,9$;

31,$2 ; 30,5 ; 21,97$

-IV (filme líquido), $v_{\max }: 3465 ; 2954 ; 1463 ; 1376 ; 1186 ; 1071 \mathrm{~cm}^{-1}$.

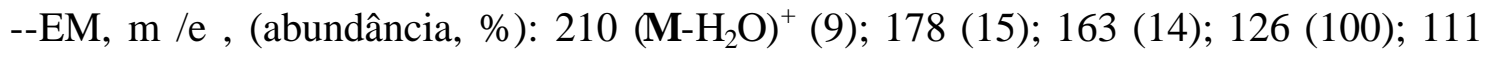
(73); 83 (46); 69 (35); 43 (42). 


\section{REFERÊNCIAS BIBLIOGRÁFICAS}




\section{REFERÊNCIAS BIBLIOGRÁFICAS}

${ }^{1}$ Constantino, M. G.; Matias, L. G. O.; da Silva, G. V. J.; Heleno, V. C. G.; Gambardella, M. T. P. Synth. Commun. 1997, 27, 4285.

${ }^{2}$ Carvalho, I.; Constantino, M. G.; da Silva, G. V. J.; Archanjo, F. C. Molecules 1996, 1,72 .

${ }^{3}$ Lacerda Jr., V.; Aragão, V.; Constantino, M. G.; da Silva, G. V. J. $22^{\underline{a}}$ Reunião Anual da SBQ, Resumos, QO-147, 1999.

4 Constantino, M. G.; Matias, L. G. O.; da Silva, G. V. J.; Barbieri, E.; Gambardella, M. T. P. Química Nova 1998, 21, 719.

${ }^{5}$ Heikkinen, S.; Mesilaakso, M.; Rahkama, E. Magn. Reson. Chem. 1998, 36, 627.

${ }^{6}$ Otter, A.; Bundle, D. R. J. Magn. Reson. B 1995, 109, 194.

${ }^{7}$ Schoder, H.; Haslinger, E. Magn. Reson. Chem. 1994, 32, 12.

${ }^{8}$ Braun, S.; Kalinowski, H. O.; Berger, S. 100 and Basic NMR Experiments, VCH, New York, 1996.

${ }^{9}$ Ohyama, M.; Tanaka, T.; Iinuna, M. Chem. Pharm. Bul. 1998, 46(4), 663.

${ }^{10}$ Summers, M. F.; Marzilli, L. G.; Bax, A. J. Am. Chem. Soc. 1986, 108, 4285.

${ }^{11}$ Bax, A.; Griffey, R. H.; Hawkins, B. L. J. Magn. Reson. 1983, 55 , 301.

${ }^{12}$ Constantino, M. G.; da Silva, G. V. J. Tetrahedron 1998, 54, 11363.

13 Thomas, W. A. Prog. n.m.r. spectrosc. 1997, 30, 183.

${ }^{14}$ Gunther, H. NMR spectroscopy: an introdu ction, p. 406, John Wiley \& Sons, New York, 1994.

${ }^{15}$ Luiz Gonzaga de Oliveira Matias, "Síntese de $\beta$-Hidróxi- e $\beta$-Etóxi-lactonas", tese de Doutoramento, IQ-USP, São Paulo, 1995.

16 House, H. O. Modern synthetic reactions, p. 54-67, $2^{\text {nd }}$ Edition, Benjamim/Cummings, Menlo Park, 1972.

${ }^{17}$ Fieser, L. F.; Fieser, M. Reagents for Organic Synthesis, John Wiley \& Sons, New York, 1967, Vol. 1, 958. 
${ }^{18}$ Zhdanov, R. I.; Zhenodarova, S. M. Synthesis, 1975, 222.

19 March, J., "Advanced Organic Chemistry" , 4 $4^{\text {th }}$ Edition, John Wiley \& Sons, New York, 1985.

${ }^{20}$ Huet, F.; Lechevaliera, A.; Pellet, M.; Conia, A. Synthesis 1977, 63.

21 Haines, A. H. Methods for the Oxidation of Organic Compounds; 255, Academic Press: London, 1988.

${ }^{22}$ Korte, F.; Zschocke, A. Tetrahedron 1958, 6, 201.

${ }^{23}$ Sanders, J. K. M.; Hunter, B. K. Modern NMR spectroscopy: a guide for chemists, 2ª edição, Oxford University Press Inc., New York, 1993, 104-113, 160-176.

${ }^{24}$ Derome, A. E. Modern NMR Techniques for Chemistry Research, Pergamon, Oxford, 1991, 270-275.

${ }^{25}$ Gunther, H. NMR spectroscopy: basic principles, concepts, and application in chemistry, 2ª edição, 1994.

${ }^{26}$ Borges, D. A.; Beatriz, A.; Constantino, M. G. 22 $2^{\underline{a}}$ Reunião Anual da SBQ, Resumos, QO-79, 1999.

${ }^{27}$ Daniel Frederico, "Síntese Total da Capsorubina", tese de Doutoramento, DQFFCLRP-USP, em andamento.

${ }^{28}$ PCMODEL, version 3.2, Serena Software, P. O. Box 3076, Bloomington, IN 474-23076

${ }^{29}$ PCMODEL, version 6.0, Serena Software, P. O. Box 3076, Bloomington, IN 474-23076

${ }^{30}$ PCMODEL, version 7.0, Serena Software, P. O. Box 3076, Bloomington, IN 474-23076

${ }^{31}$ GMMX, version 1.5, Serena Software, P. O. Box 3076, Bloomington, IN 47423076

${ }^{32}$ Gaussian 98, Revision A.6, M. J. Frisch, G. W. Trucks, H. B. Schlegel, G. E. Scuseria,M. A. Robb, J. R. Cheeseman, V. G. Zakrzewski, J. A. Montgomery, Jr.,R. E. Stratmann, J. C. Burant, S. Dapprich, J. M. Millam,A. D. Daniels, K. N. Kudin, M. C. Strain, O. Farkas, J. Tomasi,V. Barone, M. Cossi, R. Cammi, B. Mennucci, C. Pomelli, C. Adamo, S. Clifford, J. Ochterski, G. A. Petersson, P. Y. Ayala, Q. Cui, K. Morokuma, D. K. Malick, A. D. Rabuck, K. Raghavachari, 
J. B. Foresman, J. Cioslowski, J. V. Ortiz, B. B. Stefanov, G. Liu,A. Liashenko, P. Piskorz, I. Komaromi, R. Gomperts, R. L. Martin, D. J. Fox, T. Keith, M. A. Al-Laham, C. Y. Peng, A. Nanayakkara, C. Gonzalez, M. Challacombe, P. M. W. Gill, B. Johnson, W. Chen,M. W. Wong, J. L. Andres, C. Gonzalez, M. HeadGordon, E. S. Replogle, and J. A. Pople,Gaussian, Inc., Pittsburgh PA, 1998.

${ }^{33}$ CS MOPAC PRO ${ }^{\mathrm{TM}}$, CambridgeSoft Corporation 875 Massachusetts Avenue Cambridge, MA 02139, 1997.

${ }^{34}$ Smith, W. B. Introduction to theoretical chemistry and molecular modeling, VCH Publischers Inc, New York ,1996, 115-175.

35 Boltz 1.0, programa desenvolvido em nosso laboratório (Laboratório de Síntese Orgânica-USP/RP).

${ }^{36}$ Karplus, M. J. Amer. Chem. Soc. 1963, 85, 2870.

${ }^{37}$ Barfield, M.; Smith, W. B. J. Amer. Chem. Soc. 1992, 114, 1574.

${ }^{38}$ Haasnoot, C. A. G.; de Leeuw, F. A. A. M.; Altona, C. Tetrahedron 1980, 36, 2783.

39 Jackman, L. M.; Sternhell, S.; Applications of Nuclear Magnetic Resonance Spectroscopy in Organic Chemistry, 2- edição, Pergamon Press Ltd: Oxford, 1969.

${ }^{40}$ Perrin, D. D.; Armarego, W. L F.; Perrin, D. R.; Purification of Laboratory Chemicals, 2ª edição, Pergamon Press Ltd: Oxford, 1980. 